\title{
Microstructural evolution of Mn-based maraging steels and their influences on mechanical properties
}

\author{
By
}

Feng Qian

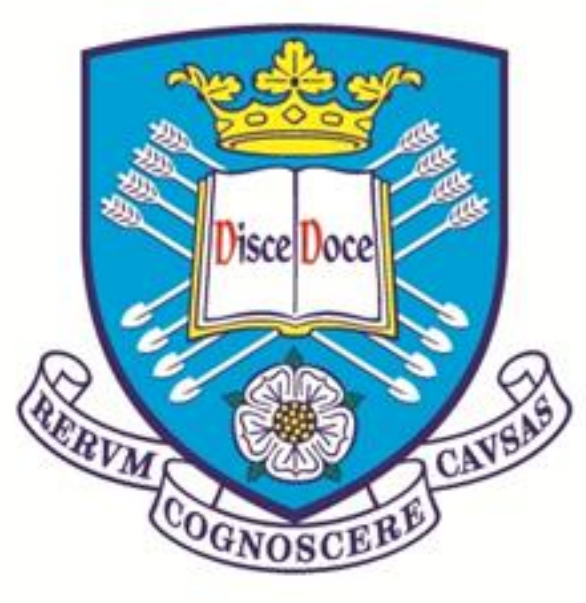

Thesis submitted for the degree of Doctor of Philosophy

Department of Materials Science \& Engineering

The University of Sheffield

March 2015 


\section{Abstract}

A set of Mn-based maraging TRIP steels was designed by Max-Planck-Institut für Eisenforschung GmbH (MPIE) for light weight and safe automotive applications. According to their research, these Mn-based maraging TRIP steels exhibited a simultaneous increase in both strength and ductility upon aging. They attributed this surprising effect to the combination of precipitation strengthening mechanism and TRIP effect of reverted/retained austenite.

This thesis carried out a further study on this type of steels with minor modification of chemical composition (7-12 wt.\% Mn, with additional 1 wt.\% Al). The unknown precipitates were characterized as $\mathrm{L} 2{ }_{1}$-ordered $\mathrm{Ni}_{2} \mathrm{TiAl}$ intermetallic phase for the first time. This type of precipitates is not only coherent but also coplanar with the martensite matrix. Their special orientation relationship together with the small lattice misfit $(1.24 \%)$ led to the precipitates remaining coherent with the martensite matrix even after a long-term aging for $10080 \mathrm{~min}$. Analyses on precipitate size revealed that the coarsening rate constants follows the diffusion-controlled coarsening kinetics form $\bar{r}^{3} \sim K t$ predicted by LSW theory, but the experimental precipitate size distributions (PSDs) is much broader than the theoretical PSD function. In addition, a core/shell structure was observed within the precipitates, but the exact structure of this structure is still not clear.

The formation of reverted austenite nanolayers initiated at the onset of aging by a diffusionless shear mechanism since the critical Mn concentration for austenite reversion at the interface is very low. The accumulated Mn segregation at grain boundaries in the following aging led to the austenite nanolayers that grew to lathlike reverted austenite, which means the lateral growth of austenite was supported by the diffusion of Mn. Due to the low diffusion rate of $\mathrm{Mn}$ and the thermodynamic resistance to coalescence, the growth rate of lath-like reverted austenite is slow and thus the austenite maintained in the range $70-200 \mathrm{~nm}$ for a long time. The segregation of $\mathrm{Ti}$ and Mo on grain boundaries in the initial aging stage resulted in the Mn concentration of austenite nanolayers being far from that indicated by the equilibrium Fe-Mn phase diagram. The segregation of Ti and Mo gradually vanished 


\begin{abstract}
with the enrichment of Mn during the succeeding aging process. The TEM-EDS analyses revealed the Mn concentration of lath-like austenite was at the level of $~ 24$ at.\% which is higher than that of retained austenite (8-12 at.\%) reported in conventional Mn-based TRIP or Q\&P steels. Nanoindentation testing revealed that the high stability of reverted austenite in Mn-based maraging steels was mainly attributed to the high $\mathrm{Mn}$ concentration of austenite. The nano-size of reverted austenite was also considered to be responsible for the high stability.

Severe embrittlement occurred in samples aged at lower temperatures or for short times. Increasing aging temperatures and duration can significantly improve the embrittlement phenomena. An ultimate tensile strength (UTS) of $1120 \mathrm{MPa}$ with total elongation (TE) of $18.4 \%$ was obtained in the $12 \% \mathrm{Mn}$ alloy by aging at $500{ }^{\circ} \mathrm{C}$ for $5760 \mathrm{~min}$. It was demonstrated that the dense precipitates contributed to the increase in yield strength whereas the work hardening of reverted austenite contributed to the enhanced strength and ductility after yielding. The TRIP effect of reverted austenite reported by Raabe et al. does not occur to any significant amount owing to the high stability of reverted austenite in Mn-based maraging steels.
\end{abstract}




\section{Acknowledgements}

First of all, I would like to express my sincere gratitude to Professor W. Mark Rainforth, for his support and supervision throughout my $\mathrm{PhD}$ study. Although he is very busy with teaching, research and administration jobs, Professor Mark Rainforth is always inspiriting to me. He is really good at encouraging students and has given me lots of freedom to realize my idea. Moreover, he is always enthusiastic to share the latest news and information in the materials world which largely broaden my vision. I also admire his remarkable knowledge of electron microscopy and his tutorial on electron microscopy will definitely benefit my future career.

I greatly acknowledge University of Sheffield, Department for Business Innovation \& Skills (BIS) and China Scholarship Council (CSC) for giving me the opportunity to study in UK and their generous financial support. Another important contributor is Tata Steel who provided the materials for this project. I am very grateful to Professor Andy Howe for the delight and helpful conservations with him and his efforts on the project coordination with industry as well. Dr Eric Palmiere and Dr Martin Jackson are also appreciated for their suggestions given in the viva for annual report.

I must express my sincere appreciation to Dr Peter Kogul, Dr Peng Zeng, Dr Le Ma and Dr Cheryl Shaw for the training and technical help at Sorby Centre for Electron Microscopy and Microanalysis. They are always here when I meet problems. Thank you all. Special thanks go to Dr Joanne Sharp for her helps on precipitation characterization using high-end TEM facilities. I am also thankful for Miss Dawn Bussey's instruction on nanoindentation tests. Many thanks are given to Dr Amit Rara, Dr Andy Williams and Mrs Vanessa Dalton who helped me a lot on daily research work.

I am very lucky to meet and work with a number of kind technical staff in the Department of Materials Science and Engineering. Thanks a lot to Mr Dean Haylock, Mr Kyle Arnold, Dr Lisa Hollands, Mr Michael Bell, Mr Richard Kangley, Mr Ian Watts and Mr Stuart Bater for their day-to-day helps on various experimental works which made this thesis possible. 
I would like to thank my dear friends who helped me a lot on my research work during these years. Particular thanks are given to Dr Yonghao Gao for those heavy work and IT support. Dr Lin Sun, who helped me a lot with the MatCalc software, is also appreciated. The experience of discussing thermodynamics and TEM with $\mathrm{Mr}$ Xiaoming Yuan and Mr Dongdong Xiao across jet lag will be one of the most valuable treasures in my life. I am also grateful for the joy and happiness brought by all friends and colleagues in the department which made this journey enjoyable.

Words are not enough to convey my deepest gratitude to my family for their unconditional love and support. My open-minded parents give me their greatest patience and understanding through all these years. They give me enough freedom to do what I like and to pursue what I want. I am so proud to have them in my life. 


\section{Table of Contents}

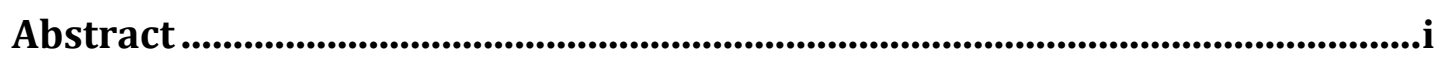

Acknowledgements.................................................................................................... iii

Table of Contents................................................................................................................

List of Figures..............................................................................................................................

List of Tables .................................................................................................................... xviii

Chapter 1 Introduction..................................................................................................................1

Chapter 2 Literature review ...................................................................................................6

2.1 The development of Mn TRIP steels...............................................................

2.2 The development of maraging steels ................................................................

2.2.1 The role of alloying elements in maraging steels .......................................

2.2.2 Behaviour of intermetallic precipitates in conventional maraging steels .. 10

2.2.3 Precipitation behaviour in newly-developed maraging steels strengthened by other intermetallic phases ......................................................................... 12

2.2.4 The Mn embrittlement in Fe-Ni-Mn and Fe-Mn alloys.............................21

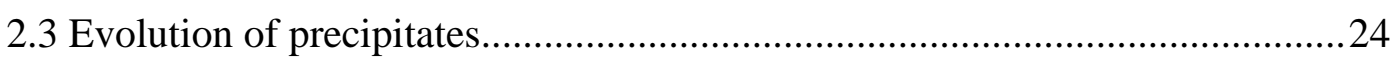

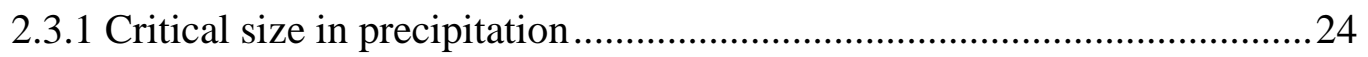

2.3.2 Growth and Coarsening of precipitates .................................................... 25

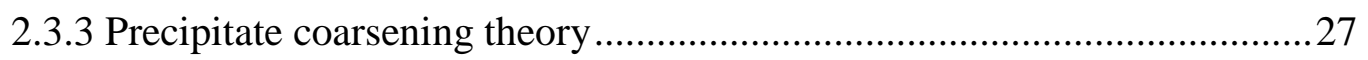

2.3.4 The effect of precipitate size on precipitation strengthening ......................29

2.4 Austenite reversion in maraging steels.............................................................. 31

2.4.1 Influence of reverted austenite on mechanical properties ............................32

2.4.2 The formation mechanism of reverted austenite..........................................41

2.5 Grain refinement in maraging steels ..............................................................4

2.5.1 Conventional methods to refine grains ......................................................4 
2.5.2 Effective grain size in lath martensitic steels. .45

2.5.3 Methods of grain refinement in lath martensitic steel ............................46

Chapter 3 Materials and Experimental Methods .....................................................48

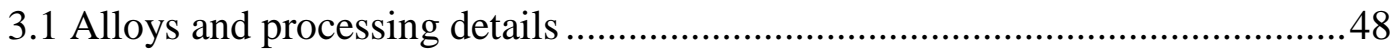

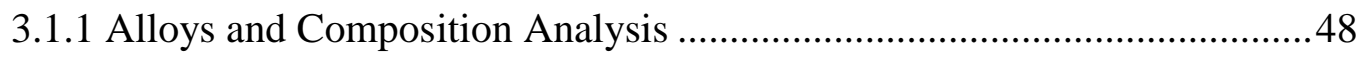

3.1.1 Hot rolling and heat treatment procedure ............................................48

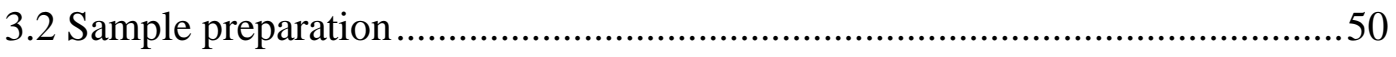

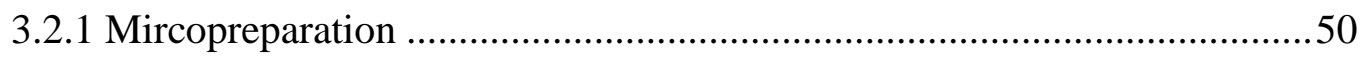

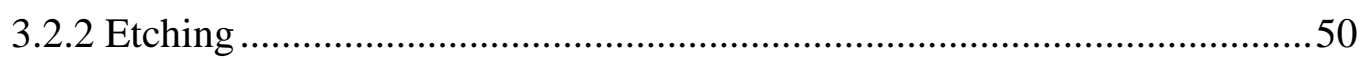

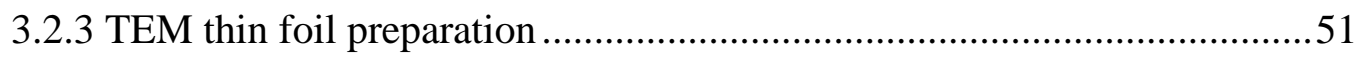

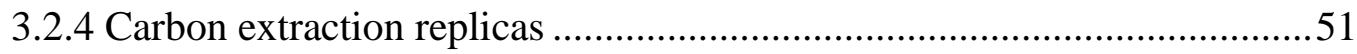

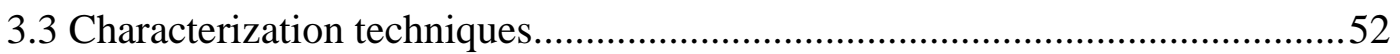

3.3.1 Qualitative and quantitative analyses by X-ray diffraction (XRD) ...........52

3.3.2 Grain size analysis by optical microscopy...........................................55

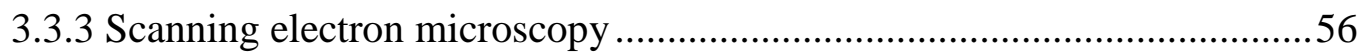

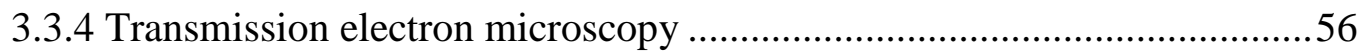

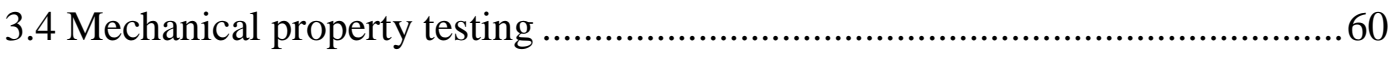

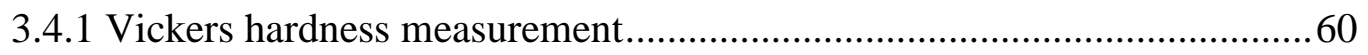

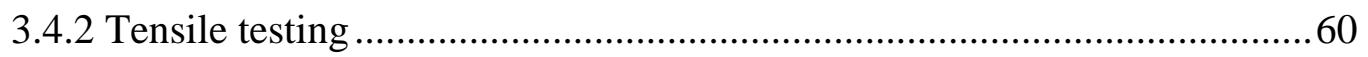

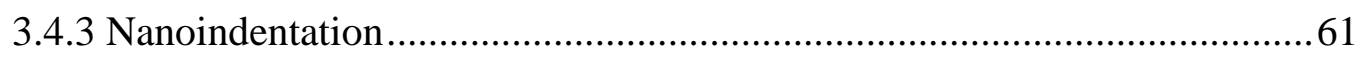

Chapter 4 Brief study on microstructure and mechanical properties of Mn-

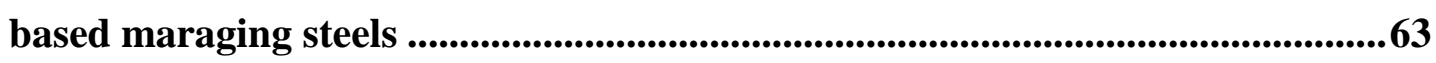

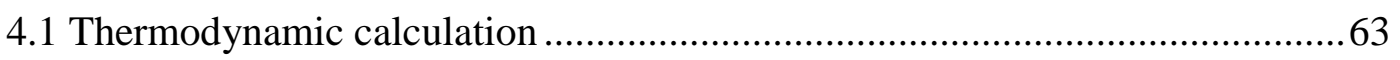

4.2 The effect of Mn content on grain refinement ...........................................65

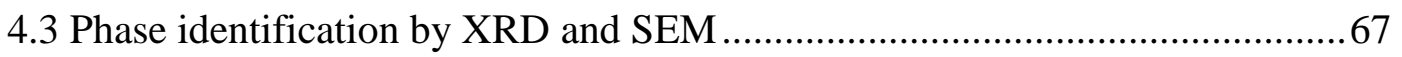

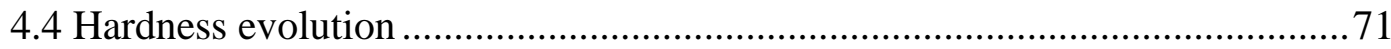




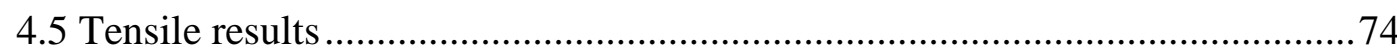

4.5.1 Tensile behaviour of solution-treated samples ...................................... 74

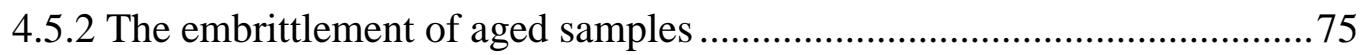

4.5.3 The improvement of mechanical properties in overaged samples.............76

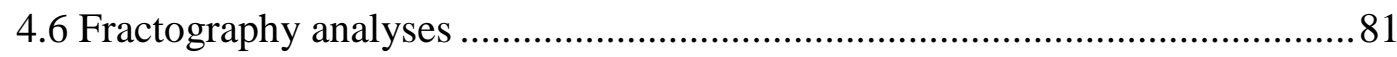

4.6.1 The fracture surface after solution heat treatment ................................ 81

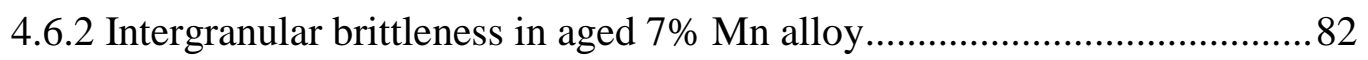

4.6.3 Improved fracture behaviour in aged 12\% Mn alloy ............................. 83

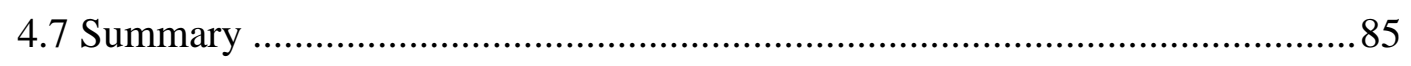

Chapter 5 Characterization of $\mathrm{L2}_{1}$-ordered $\mathrm{Ni}_{2} \mathrm{TiAl}$ intermetallic phase and its

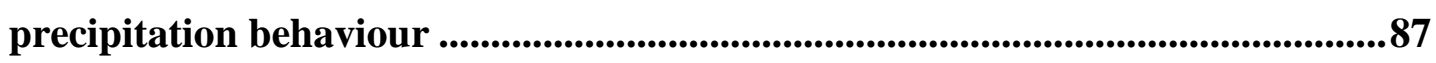

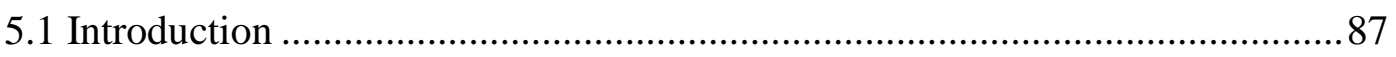

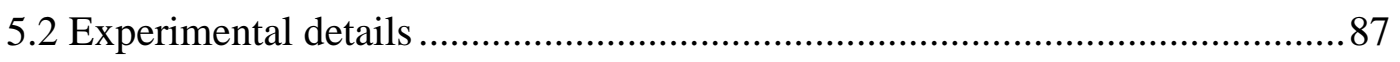

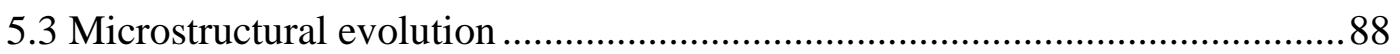

5.3.1 The effect of Mn content on the precipitation ....................................... 88

5.3.2 The effect of aging temperature on precipitation................................... 90

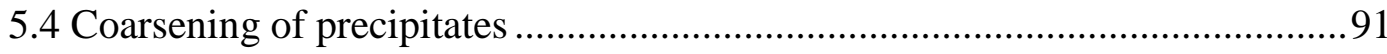

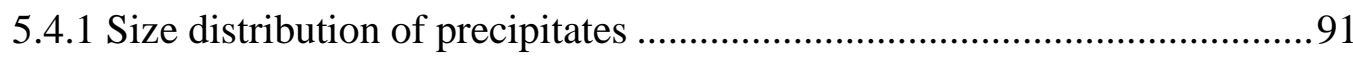

5.4.2 The growth and coarsening kinetics of precipitate .................................95

5.5 Chemical composition of precipitates …................................................. 98

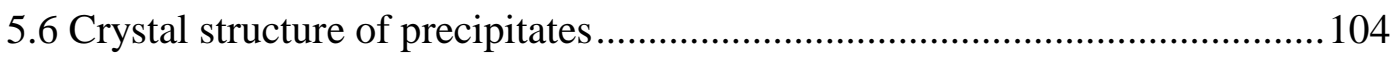

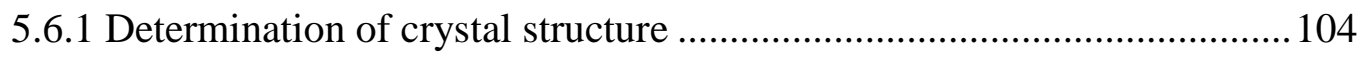

5.6.2 The orientation relationship between the precipitate and martensite matrix 110

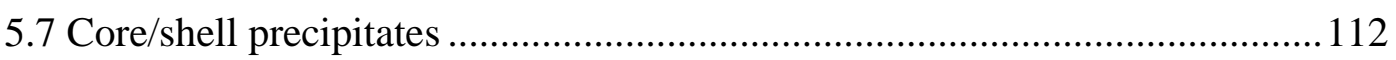

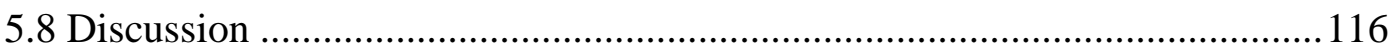


5.8.1 The effects of volume fractions on the coarsening behaviour 116

5.8.2 The formation mechanism of core/shell structure 119

5.8.3 The effect of Mn on the precipitation 122

5.9 Summary 123

Chapter 6 The formation mechanism of reverted austenite and its mechanical stability 126

6.1 Introduction 126

6.2 SEM observation 127

6.2.1 The effect of aging parameters on austenite reversion 127

6.2.2 The effect of Mn content on austenite reversion 130

6.3 TEM investigation. 131

6.4 XRD quantitative analyses 137

6.5 Nanoindentation 139

6.6 Discussion 142

6.6.1 The size of reverted austenite grains 143

6.6.2 Chemical composition of reverted austenite. 144

6.6.3 The volume fraction of reverted austenite 148

6.6.4 The formation mechanism of reverted austenite 149

6.6.5 The stability of reverted austenite 152

6.7 Summary 155

Chapter 7 Strengthening mechanisms of Mn-based maraging steels 158

7.1 The solid-solution and grain refinement strengthening........ 162

7.2 Precipitation strengthening mechanism 163

7.3 Austenite engineering in overaged stage. 166

7.3.1 The effect of reverted austenite on strengthening 167

7.3.2 The effect of reverted austenite on ductility 171 
7.4 Summary 172

Chapter 8 Conclusions and future work. 175

8.1 Phase identification 175

8.2 Hardness and tensile results 175

8.3 Precipitation behaviour of $\mathrm{L} 2{ }_{1}$-ordered $\mathrm{Ni}_{2} \mathrm{TiAl}$ phase 176

8.4 Reverted austenite and its mechanical stability 177

8.5 Optimal heat treatment suggestions 179

8.6 Future work 180

References 181

Appendix Crystallographic information of the phases in maraging steels .203 


\section{List of Figures}

Figure 1-1 Global tensile strength-elongation profile for various kinds of steels [1]..2

Figure 2-1 Stress-strain curves of samples with different heat treatment conditions in Ti-containing alloy [84]. 15

Figure 2-2 (a) An atomic resolution high-angle annular dark-field (HAADF)scanning transmission electron microscope (STEM) image along the $[001]_{\mathrm{bcc}-\mathrm{Fe}}$ zone axis; (b) magnified HAADF-STEM image of the precipitate/matrix interface in (a); (c) Dark-field image of the precipitates using the unique 111 reflection of $\mathrm{L} 21^{-}$ ordered $\mathrm{Ni}_{2} \mathrm{TiAl}$ phase where the $\mathrm{B} 2$-ordered $\mathrm{NiAl}$ zones are indicated by arrows [85]. 16

Figure 2-3 (a) Three dimensional atom probe tomographic reconstructions of rodshaped precipitates after treated for 3600 seconds and (b) corresponding $\mathrm{Ni}+\mathrm{Ti}$ isoconcentration surfaces [46]. .18

Figure 2-4 Atomic distribution of representative precipitates in the (a) $1 \mathrm{~h}$, (b) $4 \mathrm{~h}$ and (c) $1024 \mathrm{~h}$ aging stages [102]; (d) HAADF image of the core/shell precipitates and (e) Enlarged HAADF image of yellow rectangle part in (d) [45].

Figure 2-5 The evolution of precipitate size during aging in terms of precipitation kinetics, after [29].

Figure 2-6 (a) Matrix austenite at prior austenite grain boundaries [147] (b) the lamellar structure of alternate lath-like austenite and residual martensite [27] and (c) a recrystallized austenite grain free of defects [146]. .36

Figure 2-7 Different orientation relationships between fcc and bcc phases: (a) Bain, (b) K-S, and (c) N-W. Blue atoms belong to bcc unit cell and green atoms belong to fcc unit cell [179].

Figure 2-8 Schematic illustration of (a) abnormal $\alpha^{\prime} \rightarrow \gamma$ transformation and (b) normal $\alpha^{\prime} \rightarrow \gamma$ transformation [192]. 
Figure 2-9 (a) The typical microstructure of a martensitic steel constituted by packets of martensite laths, (b) the dislocated laths which is at nano-scale in thickness. Both are the micrographs of a $10 \% \mathrm{Mn}$ maraging steel after water quenching from solution heat treatment.

Figure 3-1 Schematic diagram of processing conditions

Figure 3-2 Schematic steps of making the carbon extraction replication [207]. .52

Figure 3-3 Schematic diagram of linear intercepts for the measurement of grain size.

Figure 3-4 The construction of Ewald sphere after [207].

Figure 3-5 The geometric relationship between reciprocal lattice and electron

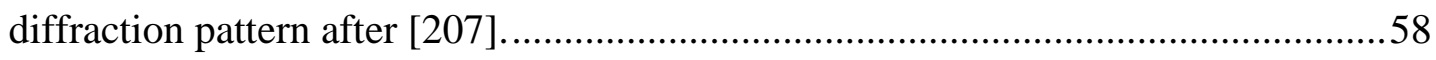

Figure 3-6 Geometry of the standard rectangular tension test sample. 61

Figure 3-7 (a) a standard Berkovich indenter where $a=65.03^{\circ}$; (b) a standard cube corner indenter where $\mathrm{a}=35.26^{\circ}$.

Figure 3-8 A typical schematic of load-displacement curve for nanoindentation test.

Figure 4-1 The equilibrium phase fractions as a function of temperature in (a) $7 \%$ Mn, (b) $10 \% \mathrm{Mn}$ and (c) $12 \%$ Mn alloys. .64

Figure 4-2 Microstructure of the three alloys in the SHT state by EBSD analyses. Left column: phase maps showing martensite phase (blue) with grain boundaries (black lines, $>15^{\circ}$ ). Right column: inverse pole maps of the same area, showing the orientation distribution of the martensite. .66

Figure 4-3 X-ray diffraction patterns of solution-treated 7\% $\mathrm{Mn}, 10 \% \mathrm{Mn}$ and $12 \%$ Mn alloys. 
Figure 4-4 (a) SEM micrograph of Ti-rich carbide present in 10\% Mn alloy in the SHT state with (b) EDS line scan and (c) EDS spot detection results; (d) TEM micrograph of Ti-rich carbide existing in aged 7\% Mn alloy in $500{ }^{\circ} \mathrm{C} / 10080 \mathrm{~min}$ state. .68

Figure 4-5 X-ray diffraction patterns of (a) 10\% Mn and (b) $12 \% \mathrm{Mn}$ alloys aged at $500{ }^{\circ} \mathrm{C}$ for various durations.

Figure 4-6 (a) Schumann's martensitic transformation diagram of the Fe-Mn system [154]; (b) schematic map of the phase fractions after cooling to room temperature based on Schumann's description [215]. .70

Figure 4-7 SEM micrographs of (a) lath-like austenite and precipitate-decorated martensite matrix and (b) homogenously distributed precipitates in $12 \% \mathrm{Mn}$ alloy in $500{ }^{\circ} \mathrm{C} / 2880 \mathrm{~min}$ state. .71

Figure 4-8 Vickers hardness as a function of aging time at (a) $420{ }^{\circ} \mathrm{C}$, (b) $460{ }^{\circ} \mathrm{C}$ and (c) $500{ }^{\circ} \mathrm{C}$ for Mn-based maraging steels, respectively.

Figure 4-9 Engineering stress vs. strain of 7\%, $10 \%$ and $12 \%$ Mn alloys in the SHT state.

Figure 4-10 (a) Engineering stress-strain curves of $10 \% \mathrm{Mn}$ alloy in $460{ }^{\circ} \mathrm{C} / 10080$ min state and $7 \% \mathrm{Mn}$ alloy in $500{ }^{\circ} \mathrm{C} / 60 \mathrm{~min}$ state with their corresponding fracture images: (b) $10 \% \mathrm{Mn}$ alloy in $460{ }^{\circ} \mathrm{C} / 10080 \mathrm{~min}$ state and (c) $7 \% \mathrm{Mn}$ alloy in $500{ }^{\circ} \mathrm{C} / 60$ min state. .76

Figure 4-11 Engineering stress vs. engineering strain of $10 \% \mathrm{Mn}$ alloy aged at $500{ }^{\circ} \mathrm{C}$ for different durations. .77

Figure 4-12 Engineering stress vs. strain of $12 \%$ Mn alloy aged at $500{ }^{\circ} \mathrm{C}$ for different durations. .78

Figure 4-13 Engineering stress-strain curves of 12\% Mn alloy aged for 10080 minutes at different temperatures. 
Figure 4-14 Engineering stress-strain curves of 7\% Mn, 10\% Mn and 12\% Mn alloys in $500{ }^{\circ} \mathrm{C} / 10080 \mathrm{~min}$ state. 80

Figure 4-15 Comparison of UTS and TE between 10\% Mn and 12\% Mn alloys aged at $500{ }^{\circ} \mathrm{C}$ for different durations.

Figure 4-16 The fracture surface of solution-treated (a) 7\% Mn; (b) 10\% Mn and (c) $12 \% \mathrm{Mn}$ alloys.

Figure 4-17 The fracture surface of 7\% Mn alloy aged at: (a) $420{ }^{\circ} \mathrm{C} / 10080 \mathrm{~min}$; (b) $460{ }^{\circ} \mathrm{C} / 10080 \mathrm{~min}$; (c) $500{ }^{\circ} \mathrm{C} / 60 \mathrm{~min}$ and (d) $500{ }^{\circ} \mathrm{C} / 10080 \mathrm{~min}$. 83

Figure 4-18 The fracture surface of $12 \%$ Mn alloys aged at $460{ }^{\circ} \mathrm{C}$ for (a)(b) 4320 min and (c)(d) $10080 \mathrm{~min}$. The right column is the enlarged fractographs. .84

Figure 4-19 The fracture surface of $12 \%$ Mn alloys aged at $500{ }^{\circ} \mathrm{C}$ for (a) $10 \mathrm{~min}$; (b) $1440 \mathrm{~min}$ and (c)(d) $10080 \mathrm{~min}$.

Figure 5-1 Bright-field TEM micrographs of precipitates formed at $500{ }^{\circ} \mathrm{C}$. Left: $7 \%$ Mn alloy for (a) $480 \mathrm{~min}$ (c) $2880 \mathrm{~min}$ and (e) $10080 \mathrm{~min}$; right: 12\% Mn alloy for (b) $480 \mathrm{~min}$ (d) $2880 \mathrm{~min}$ and (f) $10080 \mathrm{~min}$.

Figure 5-2 TEM micrographs of precipitates on carbon extraction replicas of $12 \%$ Mn alloy aged at (a) $420{ }^{\circ} \mathrm{C}$, (b) $460{ }^{\circ} \mathrm{C}$ and (c) $500{ }^{\circ} \mathrm{C}$ for $10080 \mathrm{~min}$. 91

Figure 5-3 Examples of PSDs plotted for various aged samples: 7\% Mn alloy (a) $500{ }^{\circ} \mathrm{C} / 480 \mathrm{~min}$, (b) $500{ }^{\circ} \mathrm{C} / 2880 \mathrm{~min}$ and (c) $500^{\circ} \mathrm{C} / 10080 \mathrm{~min} ; 10 \% \mathrm{Mn}$ alloy (d) $500{ }^{\circ} \mathrm{C} / 480 \mathrm{~min}$, (e) $500{ }^{\circ} \mathrm{C} / 2880 \mathrm{~min}$ and (f) $500^{\circ} \mathrm{C} / 10080 \mathrm{~min} ; 12 \% \mathrm{Mn}$ alloy (g) $500{ }^{\circ} \mathrm{C} / 480 \mathrm{~min}$, (h) $500{ }^{\circ} \mathrm{C} / 2880 \mathrm{~min}$ and (i) $500^{\circ} \mathrm{C} / 10080 \mathrm{~min}$. The histograms of the PSD function, $g$, are plotted as a function of reduced precipitate radius $(\rho=r / r)$. Theoretical LSW distribution is presented as well for comparison.

Figure 5-4 Evolution of the mean precipitate size $r$ as a function of aging time at $500{ }^{\circ} \mathrm{C}$ in (a) $7 \% \mathrm{Mn}$ alloy and (b) $12 \% \mathrm{Mn}$ alloy. .95 
Figure 5-5 Evolution of the mean precipitate radius $r 3$ as a function of aging time $t$, in $7 \% \mathrm{Mn}, 10 \% \mathrm{Mn}$ and $12 \% \mathrm{Mn}$ alloys aged at $500{ }^{\circ} \mathrm{C}$ .96

Figure 5-6 (a) Bright-field TEM image of 7\% Mn alloy aged at $500{ }^{\circ} \mathrm{C}$ for 12960 min and TEM-EDS spectra of (b) precipitate and (c) matrix.

Figure 5-7 STEM micrograph of precipitates in $12 \% \mathrm{Mn}$ alloy aged at $500{ }^{\circ} \mathrm{C}$ for $2880 \mathrm{~min}$ and EDS results of concentration profiles measured along the red line. . 100

Figure 5-8 Crystal structure of the B2-ordered NiAl phase and simulated electron diffraction patterns along low-index zone axis directions simulated using ICDD PDF-4+ software. 105

Figure 5-9 Crystal structure of the $\mathrm{L} 2_{1}$-ordered $\mathrm{Ni}_{2} \mathrm{TiAl}$ phase and its simulated electron diffraction patterns along low-index zone axis directions simulated using ICDD PDF-4+ software. 106

Figure 5-10 (a) Eight unit cells of the B2-ordered NiAl for a better comparison with (b) the unit cell of $\mathrm{L} 2_{1}$-ordered $\mathrm{Ni}_{2} \mathrm{TiAl}$; diffraction patterns along the [011] zone axis of (c) B2-ordered $\mathrm{NiAl}$ and (d) $\mathrm{L}_{2}{ }_{1}-\mathrm{Ni}_{2} \mathrm{TiAl}$ simulated using ICDD PDF-4+ software. 108

Figure 5-11 HREM micrographs of precipitates in $12 \% \mathrm{Mn}$ alloy aged at $500{ }^{\circ} \mathrm{C}$ for $10080 \mathrm{~min}$, (a) [011] zone axis; (b) IFFT of (a); (c) [111] zone axis; (d) IFFT of (c).

Figure 5-12 (a) Bright-field TEM micrograph of 7\% Mn alloy aged at $500{ }^{\circ} \mathrm{C}$ for 10080 min along [011] zone axis and (b) corresponding dark-field micrograph using the [111] superlattice spots of precipitates (inset is the corresponding diffraction pattern). 110

Figure 5-13 Selected area diffraction patterns taken on thin foil samples $\left(500{ }^{\circ} \mathrm{C} /\right.$ $10080 \mathrm{~min}, 10 \% \mathrm{Mn}$ alloy) along (a) [001], (b) [011] and (c) [111] zone axes...... 110

Figure 5-14 Two-beam bright field micrographs of (a) 7\% $\mathrm{Mn}$ and (b) $10 \% \mathrm{Mn}$ alloys after aging at $500{ }^{\circ} \mathrm{C}$ for $10080 \mathrm{~min}$. 112 
Figure 5-15 Left column: representative bright-field TEM micrographs. Right column: magnified carbon extraction replica TEM micrographs of precipitates. (a) $7 \%$ Mn alloy, $500{ }^{\circ} \mathrm{C} / 10080 \mathrm{~min}$; (b) $10 \% \mathrm{Mn}$ alloy, $500{ }^{\circ} \mathrm{C} / 10080 \mathrm{~min}$ and (c) $12 \%$ Mn alloy, $500{ }^{\circ} \mathrm{C} / 2880 \mathrm{~min}$.

Figure 5-16 HAADF image of core/shell precipitates in $12 \% \mathrm{Mn}$ alloy aged at $500{ }^{\circ} \mathrm{C}$ for $2880 \mathrm{~min}$. 114

Figure 5-17 HREM micrographs of the precipitate in $12 \% \mathrm{Mn}$ alloy aged at $500{ }^{\circ} \mathrm{C}$ for $10080 \mathrm{~min}$. (a) The low-magnification images of precipitates, (b) core/shell structure at a higher magnification and (c) lattice information at the periphery of precipitate. 115

Figure 5-18 The precipitate size distribution as a function of reduced precipitate radius $R / \bar{R}$ for different volume fractions of precipitate $f_{p}$ [226]. .117

Figure 6-1 SEM micrographs showing the morphologies of reverted austenite in the $12 \% \mathrm{Mn}$ alloy during aging. (a) Thin austenite layers formed along boundaries and (b) highly dispersed fine precipitates at $420{ }^{\circ} \mathrm{C} / 10080 \mathrm{~min}$ state; (c) evident matrix austenite and austenite layers at $460{ }^{\circ} \mathrm{C} / 2880 \mathrm{~min}$ state; (d) the amount of austenite increased after aging at $460{ }^{\circ} \mathrm{C}$ for $10080 \mathrm{~min}$. 128

Figure 6-2 (a) Fine precipitates and (b) thin matrix austenite at the onset of aging in the $12 \% \mathrm{Mn}$ alloy $\left(500{ }^{\circ} \mathrm{C} / 10 \mathrm{~min}\right.$ state); (c) the lath-like austenite became dominant after aging for $2880 \mathrm{~min}$; (d) a lamellar structure of alternative lath-like austenite and residual martensite in the 10080 min aged sample.

Figure 6-3 (a) Reverted austenite in the $12 \% \mathrm{Mn}$ alloy after aging at $540{ }^{\circ} \mathrm{C}$ for $10080 \mathrm{~min}$; (b) reverted austenite is free of precipitates. 130

Figure 6-4 SEM observation of (a) 7\% Mn alloy (500 ${ }^{\circ} \mathrm{C} / 2880 \mathrm{~min}$ state); (b) $7 \%$ Mn alloy $\left(500{ }^{\circ} \mathrm{C} / 10080 \mathrm{~min}\right.$ state); (c) $10 \% \mathrm{Mn}$ alloy $\left(500{ }^{\circ} \mathrm{C} / 2880 \mathrm{~min}\right.$ state); (d) $10 \% \mathrm{Mn}$ alloy $\left(500{ }^{\circ} \mathrm{C} / 10080 \mathrm{~min}\right.$ state). 131 
Figure 6-5 Matrix austenite formed along prior austenite grain boundaries in (a) 10\% Mn alloy at $500{ }^{\circ} \mathrm{C} / 1440 \mathrm{~min}$ state and (b) $7 \% \mathrm{Mn}$ alloy at $500{ }^{\circ} \mathrm{C} / 10080 \mathrm{~min}$ state.

Figure 6-6 TEM micrographs of lath-like austenite in (a) $12 \% \mathrm{Mn}$ alloy aging at $500{ }^{\circ} \mathrm{C}$ for $2880 \mathrm{~min}$ and (b) $12 \% \mathrm{Mn}$ alloy aging at $540{ }^{\circ} \mathrm{C}$ for $2880 \mathrm{~min}$; (c) and (d) are the corresponding images from a different orientation.

Figure 6-7 Bright-field TEM images of austenite in the martensite matrix and the corresponding selected area diffraction patterns presenting (a) K-S relationship and (b) Nishiyama relationship between austenite and martensite. 137

Figure 6-8 The evolution of volume fractions (vol.\%) of reverted austenite formed as a function of aging time at the temperature of $500{ }^{\circ} \mathrm{C}$. 138

Figure 6-9 Load-displacement curves of martensite and austenite in the $12 \% \mathrm{Mn}$ alloy in the $500{ }^{\circ} \mathrm{C} / 10080 \mathrm{~min}$ state.

Figure 6-10 Hardness distribution of austenite and martensite phases in (a) 10\% $\mathrm{Mn}$ alloy, in $500{ }^{\circ} \mathrm{C} / 10080 \mathrm{~min}$ state and (b) $12 \% \mathrm{Mn}$ alloy, in $500{ }^{\circ} \mathrm{C} / 10080 \mathrm{~min}$ state.

Figure 6-11 Load-displacement curve recording the martensitic transformation of an austenite grain.

Figure 6-12 Average width of lath-like reverted austenite after aging at $500{ }^{\circ} \mathrm{C}$ for different times in the $10 \% \mathrm{Mn}$ and $12 \%$ Mn alloys. 144

Figure 6-13 Average width of reverted austenite aged at different temperatures for $10080 \mathrm{~min}$ in the $12 \% \mathrm{Mn}$ alloy. 144

Figure 6-14 Equilibrium phase diagram of the Fe-Mn system. 146

Figure 6-15 Arrhenius plot for the determination of the activation energy of austenite formation in the $10 \% \mathrm{Mn}$ and $12 \% \mathrm{Mn}$ alloy. 151 
Figure 6-16 (a) The average hardness of austenite and martensite in the 10\% Mn and $12 \% \mathrm{Mn}$ alloys aged at $500{ }^{\circ} \mathrm{C}$ for $10080 \mathrm{~min}$; (b) the hardness of austenite and martensite in the $12 \% \mathrm{Mn}$ alloy as a function of aging temperature. 154

Figure 7-1 The optical micrographs of $7 \%$ Mn alloy (a) at SHT state and (b) $500{ }^{\circ} \mathrm{C} /$ 2880 min state. 163

Figure 7-2 (a) The engineering stress-strain curves of 12\% Mn alloy in different heat treatment conditions; (b) the corresponding hardness evolution of $12 \% \mathrm{Mn}$ alloy aged at $500{ }^{\circ} \mathrm{C}$ 165

Figure 7-3 SEM images of samples after-tensile (a) 10\% Mn and (b) 12\% Mn alloys aged at $500{ }^{\circ} \mathrm{C}$ for $5760 \mathrm{~min}$. (c) The volume fractions (vol.\%) of reverted austenite as a function of aging time at the temperature of $500{ }^{\circ} \mathrm{C}$ (solid lines: before tensile testing; dash lines: after tensile testing).

Figure 7-4 Hardness evolution vs. volume fraction of austenite as a function of time in (a) $10 \% \mathrm{Mn}$ and (b) $12 \% \mathrm{Mn}$ alloys when aged at $500{ }^{\circ} \mathrm{C}$. 168

Figure 7-5 (a) The true stress-strain curves of the 12\% Mn alloy in different heat treatment conditions; the true stress-strain curves of the $10 \% \mathrm{Mn}$ alloy in the $500{ }^{\circ} \mathrm{C}$ $15760 \mathrm{~min}$ state is also displayed for comparison. 170

Figure 7-6 SEM images of $12 \% \mathrm{Mn}$ alloy aged at $540{ }^{\circ} \mathrm{C}$ for $360 \mathrm{~min}$ : (a) reverted austenite formed along grain boundaries and (b) nano-precipitates. 174 


\section{List of Tables}

Table 2-1 Overview of the different orientation relationships between fcc and bcc crystals [168] .39

Table 3-1 Chemical compositions (wt.\%) of the investigated Mn-based steels........48

Table 3-2 Parameters for hot rolling process .49

Table 3-3 Calculated theoretical intensity ratios $\frac{R_{\gamma}^{h k l}}{R_{\alpha}^{h k l}}$ using Co $\mathrm{K}_{\alpha}$ radiation [206]..55

Table 4-1 Summary of the mechanical properties of $7 \% \mathrm{Mn}, 10 \% \mathrm{Mn}$ and $12 \% \mathrm{Mn}$ alloys in the SHT state. .75

Table 4-2 Summary of the mechanical properties of $10 \%$ Mn alloys aged at $500{ }^{\circ} \mathrm{C}$ for different durations.

Table 4-3 Summary of mechanical properties of $12 \% \mathrm{Mn}$ alloy aged at $500{ }^{\circ} \mathrm{C}$ for different durations.

Table 5-1 Experimental mean size of precipitates $(r)$ and coarsening rate constant $\left(K_{R}\right)$ of different alloys aged at $500{ }^{\circ} \mathrm{C}$.

Table 5-2 Average chemical composition in at.\% of the nano-sized precipitates evolved at $500{ }^{\circ} \mathrm{C}$ measured by TEM-EDS. 103

Table 5-3 The diffusion rates of the constituent elements in bcc-Fe at $500{ }^{\circ} \mathrm{C} \ldots \ldots .121$

Table 5-4 The mixing enthalpy of elements in the Mn-based maraging steels [238].

Table 6-1 Chemical compositions (at.\%) of reverted austenite and martensite in the $7 \% \mathrm{Mn}$ alloy obtained by TEM-EDS 135

Table 6-2 Chemical compositions (at.\%) of reverted austenite and martensite in the $12 \% \mathrm{Mn}$ alloy under various heat treatment conditions obtained by TEM-EDS. ... 136 
Table 6-3 The volume fractions (vol.\%) of reverted austenite formed in different aging conditions.

Table 6-4 A summary of the equilibrium Mn concentration (at.\%) and volume fraction (vol.\%) of austenite and ferrite phases at different aging temperatures based on the Fe-Mn phase diagram in Figure 6-14. 148

Table 7-1 Summary of the tensile properties of Mn-based steels. 159

Table 7-2 Lattice parameter ( $\mathrm{A})$ of $\alpha^{\prime}$-martensite in the three alloy in different heat treatment conditions. 162 


\section{Chapter 1 Introduction}

Steels possessing a good balance of mechanical properties (tensile strength $\times$ elongation) are of great importance to their applications. Specifically in the automotive industry, they are regarded as the future key materials for light-weight strategies and related fuel and car emissions savings. The huge commercial demand therefore has driven both industry and academia to make great progress in developing various high strength steel (HSS) grades and corresponding processing technology.

High-strength low-alloy (HSLA) steels, as the most conventional HSS group, have a microstructure of fine ferrite grains strengthened by carbides and/or nitrides of $\mathrm{Ti}, \mathrm{V}$ or $\mathrm{Nb}$. Generally their strength is at the level of 700-800 MPa. As this type of steels can be manufactured by simple processing paths, they have been widely used in automotive applications. In order to further improve the combining properties of strength and ductility, other alloying elements have been added and hence more sophisticated alloy systems have been designed, such as Dual Phase (DP) steels, Transformation Induced Plasticity (TRIP) steels, Twinning Induced Plasticity (TWIP) steels and martensitic (MART) steels. Global tensile strength-elongation profile of those steel families is displayed in Figure 1-1 [1]. Dual phase steels are mainly constituted of soft ferrite with dispersed islands of hard martensite. Their strength is dependent on the amount of martensite and its morphology and distribution [2][3][4][5]. TRIP steels are multiphase grades which utilize specially designed alloying additions and processing paths to retain certain amount of austenite down to room temperature within a ferritic matrix. This metastable austenite transforms to martensite when subjected to plastic deformation, which leads to a volume and shape change of the resulting martensite and matrix to accommodate the transformation misfit and thus increases the ductility [6][7][8]. TWIP steels normally have a fully austenitic microstructure at room temperature. The formation of mechanical twins of austenite during deformation induces high strain hardening and prevents necking, which maintains a very high strain capacity and achieves a better balance of strength and ductility [9][10][11]. Another group of HSS is martensitic (MS) steels, among 
which maraging steels is the most widely studied category. Maraging steels are known for its ultrahigh strength which is attributed to the heavily strained martensite matrix upon quenching and further precipitation strengthening by aging treatment. These precipitates act as highly efficient obstacles against dislocation motion via Orowan mechanism. The detailed microstructure and properties of maraging steels will be introduced in Section 2.2.

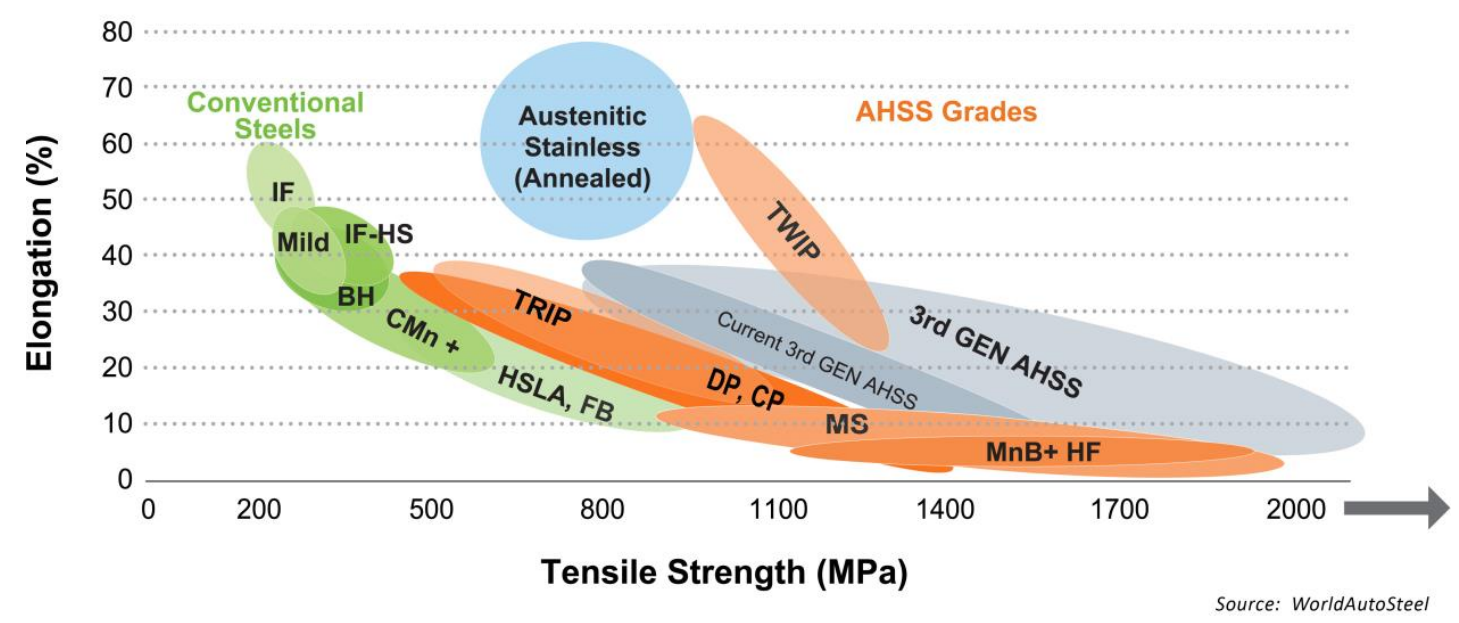

Figure 1-1 Global tensile strength-elongation profile for various kinds of steels [1].

It is recognized that deformation-induced martensitic transformation plays an important role in improving the mechanical properties of TRIP steels. Conventionally, high level of carbon content is added to retain austenite at room temperature, but the poor weldability resulting from high carbon addition restricts its application in the automotive industry. Hence, $\mathrm{Mn}$, as another effective austenite stabilizer, is used to substitute carbon. In 1960s, Goldshtein et al. first reported an excellent toughness obtained in a low-carbon TRIP steel $(0.1 \mathrm{wt} \% \mathrm{C}, 8$ wt.\% Mn) with a considerable amount of austenite [12]. Since then many researches on medium Mn steels have been carried out [13][14][15][16]. A recent study by Luo et al. [17] reported a 5Mn steel, which contained more than 30 vol.\% austenite, exhibited an ultimate tensile strength (UTS) of 1-1.5 GPa with total elongation (TE) of $31-44 \%$.

On the other hand, it is generally accepted that the superior mechanical properties in maraging steels are attributed to the formation of strengthening nano-precipitates 
upon aging. In fact, as the aging process is normally performed at the two-phase field, another phase transformation reaction, the partial reversion of martensite to austenite, is expected to occur. Evidence has demonstrated that austenite reversion can significantly affect the mechanical properties [18][19][20]. The most classical maraging group is nickel-based maraging steels (18 wt.\% Ni) with considerable additions of elements such as $\mathrm{Co}(8 \mathrm{wt} . \%), \mathrm{Ti}, \mathrm{Cr}$ and Mo. Owing to the sharp drop of the availability of Co since the 1960s, many researchers have focused on developing alternatives to expensive $\mathrm{Co}$ and $\mathrm{Ni}$ containing maraging steels. Among them, studies on Mn-based maraging steels have made great progress in minimising the strength sacrifice resulting from the absence of Co and Ni.

Based on the above discussion, the strategy of combining the two strengthening mechanisms in the form of Fe-Mn alloys was proposed. According to this strategy, a microstructure including hard martensite strengthened by nano-precipitates and ductile austenite in which transformation-induced plasticity can occur should be attained. Raabe et al. [18] designed a type of low carbon content (0.01 wt.\% C), 9-12 wt.\% Mn steels with minor additions of Ni, Ti and Mo (1-2 wt.\%). Contradictory to the common knowledge that the increase in strength is accompanied by a decrease in ductility (Figure 1-1), those steels exhibited a simultaneous increase in strength and ductility. A UTS of $\sim 1300 \mathrm{MPa}$ with a tensile elongation of $21 \%$ was reported in a 12 wt.\% Mn maraging TRIP steel.

This great achievement opens a new approach to develop ultra-high steels at relatively lean alloying costs. However, there are still some unsolved questions about this new type of steels, e.g. the nature and composition of precipitates are debated. The respective formation mechanisms governing the austenite reversion and precipitation are not clear. Given that the chemical composition, morphology and grain size of reverted austenite are different from those of retained austenite, the deformation mechanism of reverted austenite in these steels is supposed to be different from that of retained austenite. The strengthening mechanism of this type of steels is also worth studying. Therefore, it is necessary to carry out a detailed study on this type of steels and based on the study, the optimal microstructure and related processing paths can be proposed. 
The present work is undertaken in collaboration with Tata Steel. The aim was to proceed with a further study on a series of $7-12 \% \mathrm{Mn}$ maraging steels. Characterization of the unknown precipitates is the primary task of this work. Then the formation mechanism of precipitates and reverted austenite are discussed, respectively. Based on the investigation on the strengthening mechanisms, optimal chemical composition and processing techniques are determined.

This thesis continues with Chapter 2 which presents a general survey of relevant literature for the study on Mn-based maraging steels. After the introduction on the development of Mn TRIP steels and Mn maraging steels, various precipitation behaviours in different maraging steels are reviewed. Subsequently, the basic knowledge about precipitate coarsening is given. Then, the discussion on the mechanical stability of austenite and its formation mechanism is followed by a brief overview of grain refinement in maraging steels.

Chapter 3 provides the information about the materials studied in this thesis and the relevant processing paths. Various sample preparation methods and characterization techniques are also described.

Chapter 4 presents the results of some basic analyses on these steels to give the most straightforward understanding about Mn-based maraging steels, e.g. the thermodynamic calculation results, phase identification by X-ray diffraction (XRD), hardness and tensile testing results, etc.

In Chapter 5, microstructural evolution of nano-scale precipitates during aging is described. Based on the observation, the precipitate coarsening kinetics is discussed. Then the chemical composition and crystal structure of precipitates are studied to identify the precipitates. In addition, their orientation relationship with martensite matrix and the core/shell structure are also investigated.

Chapter 6 mainly focuses on the austenite reversion in maraging steels. Apart from the microstructural observation on reverted austenite in various aging conditions, the quantity and chemical composition of reverted austenite are measured to provide information about its mechanical stability. Then, the analyses of nanoindentation results of different samples are given. Based on these results, the factors which are normally considered to affect the mechanical stability of reverted austenite, such as 
morphology, orientation relationship, grain size and chemical composition etc., are discussed successively.

After a brief comparison of the mechanical properties of Mn-based maraging steels in this work with other medium Mn steels reported in the literature, the focus of Chapter 7 is concerned with the definition of strengthening mechanisms mainly involving solid-solution strengthening, grain-refinement strengthening, precipitation strengthening and phase transformation strengthening mechanisms (TRIP effect).

Chapter 8 draws conclusions of this thesis based on the results and discussion of all chapters and future work are proposed as well. 


\section{Chapter 2 Literature review}

\subsection{The development of Mn TRIP steels}

Two primary motivations have been driving the development of advanced high strength steel (AHSS) for automotive applications. The first motivation is to decrease the consumption of fuel and car emissions by mass reduction. The second is the increasing requirement for comfort, safety and speed of vehicles. From this point of view, AHSSs with high strength to weight ratio are the key materials for automotive manufactures [1][21][22]. The first-generation automotive-grade AHSSs (Figure 1-1), such as DP steels, TRIP steels and complex-phase (CP) steels which possess multi-phase microstructures comprised of different percentages of bainite, martensite and carbon-rich austenite, have been extensively investigated in the past [1][22]. The second-generation AHSSs are the twinning-induced plasticity (TWIP) steels. These high Mn (> 15 wt.\%) austenitic-based steels yield a combination of strength and ductility superior than those of the first-generation AHSSs [10][11], but the high alloying additions (e.g. $\mathrm{Mn}, \mathrm{Al}$ and $\mathrm{C}$ ) and complicated processing technology limited their application in automotive industry [23]. Recently, the development of third-generation AHSSs with medium Mn contents (5-12 wt.\%) have been designed and developed. In these steels, varying fractions of metastable austenite with fine grain size were produced by adjusted intercritical tempering [16][24][25][26]. It is reported that the medium Mn steels containing sub-micro austenite grains can achieve a high ultimate tensile strength and excellent total elongation [27][22][26]. This is because the plasticity of the austenite is not only dependent on the mobile dislocations, two other plastic modes, i.e., strain-induced martensitic transformation and mechanical twinning, are also involved in the deformation of austenite. The operation of specific deformation modes is determined by the microstructural characteristics of austenite (which will be discussed in Section 2.4). This feature is significant in the design of medium Mn steels containing austenite. Recent studies on 5-7 wt.\% Mn steels revealed that elongation of up to $45 \%$ can be achieved by optimized intercritical tempering. However, so far the yield 
strength of this steel grade hardly goes beyond $1 \mathrm{GPa}$, which obviously cannot meet the demand for the various intrusion application of hole-expanded components in automotive industry [26]. Therefore, strategies to improve the strength of these steels in which the excellent ductility can be retained are the focus of current study.

\subsection{The development of maraging steels}

Maraging steels are a special group of ultrahigh-strength martensitic steels which are hardened by intermetallic precipitates instead of universal carbide precipitates. Therefore, the carbon content in maraging steels is controlled to be as low as is commercially practicable [28]. The term 'maraging' refers to the aging of martensite. The supersaturated martensite is obtained by fast cooling from the austenite phase field and the martensite with low carbon content is very soft but heavily dislocated. This microstructural feature is believed to benefit the hardenability, toughness and formability before aging [29], and more importantly, provide an excellent nucleation condition for precipitation. The subsequent aging treatment leads to the formation of intermetallic precipitates. The nature of precipitates largely depends on the alloying compositions and aging parameters. The detailed discussion about the precipitation in maraging steels will be given in Section 2.2.3. On the other hand, the excessive softening of martensite matrix by recovery during aging should be avoided if a good balance between strength and toughness is required.

The development of maraging steels initiated in 1962 with the publication of a paper by Decker et al. [30] on 18 wt.\% Ni steels containing significant levels of Co (8 wt.\%) and Mo (5 wt.\%) but low carbon content (0.01-0.03 wt.\%). According to their report, tensile strengths of up to $2068 \mathrm{MPa}, 12 \%$ elongation, $60 \%$ reduction in area, low ductile-brittle transition temperature (DBTT) and notch tensile strengths of $\sim 3034 \mathrm{MPa}$ were achieved. Additionally, excellent stress-corrosion resistance and weldability were inherent in these alloys [30]. The following researches led to the development of the well-known maraging systems, such as $18 \mathrm{Ni}(200$ (ksi)), $18 \mathrm{Ni}$ (250) and 18Ni (300) alloys. However, the costly alloying additions of maraging steels restricted their applications in the most critical industry. The sharp drop of the availability of cobalt during the period 1978-1980 promoted the exploitation of 
alternatives to Co-containing maraging steels. Intensive efforts were directed at reducing the concentration of expensive alloying elements, such as Co and $\mathrm{Ni}$. As a result, a new class of Co-free maraging steels containing Fe, Ni, Mo and Ti emerged. These Co-free steels generally presented inferior mechanical properties compared to Co-containing steels, but their mechanical properties appeared sufficient for typical applications of maraging steels [31][32]. Moreover, due to the similarity of the equilibrium phase diagrams between $\mathrm{Fe}-\mathrm{Ni}$ and $\mathrm{Fe}-\mathrm{Mn}$ systems, the partial substitution of Ni by Mn was applied in the development of lean maraging steels. An excellent combination of strength and ductility was reported in an Fe-20.8Ni2.13Mn-0.8Ti maraging steel after aging at $550{ }^{\circ} \mathrm{C}$ for $1 \mathrm{~h}$ which exhibited a yield strength (YS: $1371 \mathrm{MPa}$ ) and total elongation (TE: 18.8\%) [33]. It should be noted, however, steels with high Mn contents were found to suffer from embrittlement after aging for short times.

The main strength of maraging steels is generally attributed to the dense precipitation of fine intermetallic compounds in a soft but heavily dislocated martensite matrix. In some cases, reverted austenite may form when aging at high temperatures or for a long period. The effect of reverted austenite on the mechanical properties is controversial. It is generally accepted that the toughness of quenching and partitioning (Q\&P) steels for low-temperature service can be improved via the formation of metastable reverted austenite after fast cooling from intercritical tempering (holding in the temperature range where the austenite and ferrite phases coexist) [27][34][26]. However, in maraging steels, different opinions exist on the effect of reverted austenite on mechanical properties (which will discuss in detail in Section 2.4.1) [19][35][36][37].

Maraging steels are known for their excellent combination of ultrahigh strength with an acceptable ductility. Due to the low carbon content, they exhibit excellent machinability and weldability in the solution-treated condition. Besides, they also possess high strength to weight ratio and dimensional stability during aging. All of these attractive properties make maraging steels as a class of promising materials and numerous researches have been carried out on the development of maraging steels. More recently, a newly-developed 12 wt.\% Mn maraging TRIP steel with minor addition of $\mathrm{Ni}, \mathrm{Ti}, \mathrm{Mo}$ and $\mathrm{Al}$ was reported to possess an excellent combination of 
strength and ductility (UTS (ultimate tensile strength): $1.3 \mathrm{GPa}$, TE (total elongation): $21 \%$ ) due to precipitation strengthening mechanism and TRIP effect of austenite. But there are some unsolved questions about this new materials, i.e. the nature of precipitates, the formation mechanism of retained/reverted austenite and its contribution to strengthening [18].

\subsubsection{The role of alloying elements in maraging steels}

The high $\mathrm{Ni}$ content in maraging steels, which significantly lowers both the equilibrium $\gamma / \alpha$ transformation temperature $\left(A_{e 3}\right)$ and martensite start temperature $\left(M_{S}\right)$, ensures the formation of martensite after quenching from solid-solution treatment. Precipitation strengthening by aging is subsequently acquired via aging at 480-510 ${ }^{\circ} \mathrm{C}$ for a range of times [29]. $\mathrm{Ni}, \mathrm{Co}, \mathrm{Ti}$, and Mo are normally added as essential elements to generate intermetallic precipitates. In recent studies on $\mathrm{Cu}$ bearing maraging steels, $\mathrm{Cu}$ precipitates were demonstrated to accelerate the nucleation of intermetallic precipitates [38][39][40]. In some maraging steels, the additions of high levels of $\mathrm{Cr}$ are required to achieve effective aging hardening in stainless grades resistant to corrosion [41][42][43]. As mentioned above, the price jump of $\mathrm{Co}$ and $\mathrm{Ni}$ drove extensive researches to develop lean maraging steels. In recent years, the development of lean maraging steels by substituting $\mathrm{Ni}$ with cheaper elements such as Mn (the price of Ni: 14410 USD/t, Co: 27750 USD/t, Mn: 2200 USD/t by Aug 2013) has made remarkable progress. As an alternative austenite stabilizer, Mn can take over the role of Ni in conventional maraging steels. Besides, experimental evidence indicated that $\mathrm{Mn}$ was also involved in the precipitation of Mn-containing maraging steels [44][45] and Mn additions were found to accelerate the age hardening process [46]. However, high maraging strength at the expense of dramatic loss in ductility was found in several Fe-Ni-Mn steels [33][47][48]. Some researchers suggested that the segregation of Mn to grain boundaries was responsible for the embrittlement phenomena in those alloys (which will discuss in Section 2.2.4) [47][49]. While further study on these Mn-containing maraging steels revealed that the embrittlement vanished by proceeding segregation of $\mathrm{Mn}$ at grain boundaries leading to the austenite reversion [50]. 


\subsubsection{Behaviour of intermetallic precipitates in conventional maraging steels}

Since the development of maraging steels in the 1960s, numerous researches have been carried out on the precipitation behaviour in this steel grade. Thanks to the development of advanced characterization techniques, such as high resolution electron microscopy (HREM) and atom probe tomography (APT) which enables the characterization of extremely fine precipitates, the nature and composition of those intermetallic precipitates in maraging steels have been generally understood. Most of studies agreed that in conventional $18 \mathrm{Ni}$ maraging steels, $\mathrm{Ni}_{3} \mathrm{Ti}(\mathrm{Mo}, \mathrm{V}, \mathrm{W})$-type phases generally appear at the very early aging stage, whereas a more stable $\mathrm{FeMo}(\mathrm{W})$-type phase is formed after long-term aging [51].

The formation of $\mathrm{N}_{3} \mathrm{Ti}(\mathrm{Mo})$-type phases at the initial aging stage in conventional maraging steels had been proposed since this type of steels was invented. The modern techniques which allow atomic-scale resolution make the direct observation on the nano-precipitates possible and finally confirm the existence of $\mathrm{Ni}_{3} \mathrm{Ti}(\mathrm{Mo})$ phase ( $\eta$-phase) in those steels. The morphology of $\mathrm{Ni}_{3} \mathrm{Ti}$ precipitates was found to be needle-like [31][52], plate-like [53] or rod-like [31]. The $\mathrm{Ni}_{3} \mathrm{Ti}$ phase exhibits hexagonal lattice with $\mathrm{a}=0.255 \mathrm{~nm}$ and $\mathrm{c}=0.42 \mathrm{~nm}$ [38]. Selected area electron diffraction (SAED) analyses indicate that the orientation relationship between $\eta$ $\mathrm{Ni}_{3} \mathrm{Ti}$ and $\alpha^{\prime}$-martensite matrix is $(011)_{\alpha^{\prime}}\left\|(0001)_{\eta},[1 \overline{1} 1]_{\alpha^{\prime}}\right\|[11 \overline{2} 0]_{\eta}[54]$. There was debate about the formation mechanism of $\mathrm{Ni}_{3} \mathrm{Ti}$ phase in the literature. Most studies suggested the heterogeneous nucleation on dislocations followed by growth via pipe diffusion [48][54]. Other researchers insisted that the precipitate nucleation occurs homogeneously, or at larger undercooling, by spinodal decomposition [55]. There were also divergent opinions about the dominant strengthening effect of each type of precipitates in these steels. Some studies revealed that in maraging steels where both $\mathrm{Ti}$ and Mo were present, Ti was much more active in the beginning due to its rapid diffusivity in martensite at the specific aging temperature. Apart from the kinetic advantage, the smaller lattice misfit between $\mathrm{Ni}_{3} \mathrm{Ti}$ and martensite and consequently a lower barrier for nucleation is another reason for the formation of 
$\mathrm{Ni}_{3} \mathrm{Ti}$ in the early stage of aging (just a few minutes or even faster). Therefore, a sharp rise in hardness shortly after the onset of aging was usually observed in Ticontaining maraging steels (C-300 and T-300 steels) [51]. At this stage, Mo is more likely to be incorporated into the $\mathrm{Ni}_{3} \mathrm{Ti}$ phase and partially substitute $\mathrm{Ti}$ atoms. As the substitution is limited, there are still sufficient Mo atoms for the following formation of FeMo-type phases. Conversely, the growth of FeMo phase would consume the Mo atoms in $\mathrm{Ni}_{3} \mathrm{Ti}(\mathrm{Mo})$ phase and therefore the stoichiometry of $\mathrm{Ni}_{3} \mathrm{Ti}(\mathrm{Mo})$ becomes more closer to that of $\mathrm{Ni}_{3} \mathrm{Ti}$. The activity of Mo has been shown to be strongly affected by other elements. The presence of Co is generally found to promote the formation of Mo-rich precipitates. When Co is absent, the driving force for the precipitation of Mo-rich precipitate is significantly reduced. In this case, the precipitation of Mo-rich phases would take 3 to 8 hours to occur [56]. Thus the major precipitates in Co-free maraging steels are entirely $\mathrm{Ni}_{3} \mathrm{Ti}$ phase and thus higher Ti content is required to achieve the same level of precipitation strengthening. In addition, the stoichiometry of FeMo-type phase is still debated. Previous TEM and SAED studies suggested it as $\mathrm{Fe}_{2} \mathrm{Mo}$ Laves phase [54][56], whereas the compositional result by a more recent ATP analysis corresponded to $\mathrm{Fe}_{7} \mathrm{Mo}_{6} \mu$ phase. Moreover, the possibilities of $\mathrm{FeMo}$ and $\mathrm{Fe}_{3} \mathrm{Mo}_{2}$ cannot be excluded as well.

Apart from the primary $\mathrm{Ni}_{3} \mathrm{Ti}(\mathrm{Mo})$-type and FeMo-type phase, several other intermetallic phases may also form as well due to the composition variation of maraging steels. In Ti-free maraging steels, the role of $\mathrm{Ti}$ is taken over by Mo. A precipitation sequence of $\mathrm{Ni}_{3} \mathrm{Mo}$ followed by equilibrium FeMo-type phase was reported in Ref. [56]. Besides, a type of metastable $\omega$ phase (ordered isothermal phase enriched in $\mathrm{Ni}, \mathrm{Co}$ and $\mathrm{Mo}$ ) was always generated before the formation of the more stable $\mathrm{Ni}_{3}$ Mo phase. Researches revealed that this $\omega$ phase had a higher level of coherency with the matrix and hence was easier to form [57][58]. In addition, the precipitation of $\mathrm{Ti}_{6} \mathrm{Si}_{7} \mathrm{Ni}_{16}$ (G-phase) was found to be responsible for the precipitation hardening of Cr-containing high-Si steels [59]. 


\subsubsection{Precipitation behaviour in newly-developed maraging steels strengthened by other intermetallic phases}

Maraging steels strengthened by intermetallic phases have complex precipitation behaviours, which vary with the chemical compositions. In this section, some lessknown intermetallic precipitates in steels are briefly introduced.

\subsubsection{NiAl-strengthened Fe-Cr-Ni-Al steels}

Precipitation-strengthened ferritic steels are candidate materials for applications such as high-pressure steam piping and heaters in ultra-supercritical fossil-energy power plants [60][61][62]. These applications generally require a creep strain rate $\sim 3 \times$ $10^{-11} s^{-1}$ at temperatures of $760{ }^{\circ} \mathrm{C}$ and stress of $35 \mathrm{MPa}$ [63][64]. Therefore, creep resistance is one of the most significant properties. Currently, most creep-resistant ferritic steels are strengthened by carbides. However, the coarsening of incoherent carbides in long-term high temperature application often leads to the decrease of creep resistance [65][66][67][68][69]. Consequently, a number of studies have been carried out to improve the creep properties of steels [70][71][72][73][74]. On the other hand, the investigation on the strengthening behaviour of high temperature materials suggests a uniform dispersion of coherent precipitates is beneficial to enhancing the strength [74]. The most classical group is the nickel-based superalloys where coherent $\mathrm{L1}_{2}$-ordered precipitates are generated to strengthen the fcc marix. Analogously in steels, B2-ordered (CsCl-type) NiAl phase (a $=0.2887 \mathrm{~nm})[75]$ is known to have a similar crystal structure to $\alpha$-Fe matrix $(a=0.2866 \mathrm{~nm})$ [75]. Its orientation relationship with matrix is $(100)_{\alpha}\left\|(100)_{N i A l},[001]_{\alpha}\right\|[001]_{N i A l}$, which means the NiAl phase is not only coherent but also coplanar with $\alpha$-Fe matrix [76]. NiAl-strengthened ferritic steels have been found to possess better thermal conductivity, lower thermal expansion and production cost compared to other materials applied in high temperature [77]. Besides, it is believed that the additions of $\mathrm{Al}$ which varies from several to more than $10 \%$ contributes to the feature of lower density $\left(\sim 7 \mathrm{~g} \cdot \mathrm{cm}^{-3}\right)[60]$. All these advantages allow the NiAl-strengthened ferritic steels to be nominated as a promising substitution for carbide-containing ferritic 
steels, austenitic steels and nickel superalloys applied in the high temperature field. Therefore, a series of NiAl-strengthened ferritic superalloys have been designed.

High strength and toughness with an improved creep resistance have been achieved by the formation of $\mathrm{B} 2$-ordered $\mathrm{NiAl}$ precipitates in these alloys [61][62][73][76]. Tailard [76] reported an Fe-20Cr-4 Ni-2Al alloy aged at 550-650 ${ }^{\circ} \mathrm{C}$ which hardened rapidly in the initial stage owing to the precipitation of $\mathrm{B} 2$-ordered $\mathrm{NiAl}$ phase. Maximum hardening of $\sim 420 \mathrm{HV}$ was achieved at around 1000 minutes / $550{ }^{\circ} \mathrm{C}$ followed by the decrease in hardness due to precipitate coarsening. In terms of morphology, $\mathrm{NiAl}$ phase initially remains coherent with the $\alpha$-Fe matrix and presents a spherical shape. Then it gradually transforms to a non-equiaxed cuboidal morphology up to $150 \mathrm{~nm}$ and eventually lose coherency at the dimension of 150$300 \mathrm{~nm}$ after an extremely long time aging. This continuous growth in size during aging indicates that the resistance to coarsening of NiAl phase needs to be improved.

In addition, the high volume fraction of $\mathrm{NiAl}$ precipitates leads to a better creep resistance, whereas the ductility is inversely related to the volume fraction of $\mathrm{NiAl}$ precipitates [60][61]. Moreover, the ductility is found to decrease with the increasing addition of $\mathrm{Al}$ in $\alpha-\mathrm{Fe}$ matrix. Therefore, an improved microstructure which possesses (1) a volume fraction of $20 \% \mathrm{NiAl}$ precipitates, (2) a solubility of $\mathrm{Al}$ below $4 \%$ in the $\alpha$-Fe matrix and (3) appropriate $\mathrm{Ni}$ and $\mathrm{Al}$ additions to avoid the austenite reversion, has been proposed to achieve a better balance between the creep resistance and ductility [61][62].

On the other hand, some other studies were devoted to developing potential stainless maraging steels also strengthened by $\mathrm{NiAl}$ precipitates for application in machinery, aircraft and sports fields [78][79][80][81][82]. Ultra-fine NiAl precipitates (1-6 nm) with a high number density $\left(10^{23-25} \mathrm{~m}^{-3}\right)$ were found homogenously distributed in martensite matrix at the early stage when aging at $450-620{ }^{\circ} \mathrm{C}$ [82]. The size and elemental concentrations of precipitates were found to increase moderately with the aging temperature while the number density decreased. A maximum yield strength (YS) of $\sim 1500 \mathrm{MPa}$ was reported in an Fe-13Cr-8Ni-2.5 Mo-2Al stainless maraging steel aged at $510{ }^{\circ} \mathrm{C}$ [82]. It has been demonstrated that B2-ordered NiAl phase formed in stainless maraging steels remained fully coherent with the matrix even 
after a significant coarsening [80][81][82]. According to Seetharaman's calculation, the critical diameter of losing the coherency between NiAl phase and $\alpha$-Fe matrix was $\sim 150 \mathrm{~nm}[80]$.

\subsubsection{The co-precipitation of $\mathrm{NiAl}$ and $\mathrm{Ni}_{3} \mathrm{Ti}$ phase in $\mathrm{Fe}-\mathrm{Cr}$-Ni-Ti-Al steels}

$\mathrm{Ti}$ is one of the most active elements in maraging steels due to its rapid, and strong precipitation effect during aging [56], hence a number of studies have been carried out to investigate the influence of $\mathrm{Ti}$ addition on the precipitation evolution in maraging steels [32][78][83][84]. Accelerated hardening was observed in an Fe-NiAl-Ti-Cr stainless steel aged at $525{ }^{\circ} \mathrm{C}$ where $~ 86 \%$ of the total increase in hardness was achieved within $0.25 \mathrm{~h}$ [32]. The uniformly dispersed clusters formed at the initial aging stage were enriched in $\mathrm{Ni}, \mathrm{Al}$ and $\mathrm{Ti}$. At the peak hardness $\left(525^{\circ} \mathrm{C} / 3 \mathrm{~h}\right)$, a splitting of the clusters occurred and two independent intermetallic phases, B2ordered $\mathrm{NiAl}$ phase and $\eta-\mathrm{Ni}_{3}(\mathrm{Ti}, \mathrm{Al})$ which were both responsible for precipitation hardening, were formed. In addition, the enrichment of $\mathrm{Fe}$ and $\mathrm{Cr}$ at the expense of $\mathrm{Ni}$ and $\mathrm{Al}$ was found in NiAl precipitates by Atom Probe tomography (APT), but the concentrations of $\mathrm{Fe}$ and $\mathrm{Cr}$ progressively decreased with aging time. In contrast, no substituting elements were found in $\mathrm{Ni}_{3} \mathrm{Ti}$ precipitates which indicated the $\mathrm{Ni}_{3} \mathrm{Ti}$ phase had a low solubility of other elements and maintained the chemically stability during aging [32].

Lately Leitner and co-workers further reported a supplement of Si in Fe-Cr-Ni-Ti-Al steels [78][84]. A heterogeneous nucleation sequence at dislocation lines was found in the very beginning of aging which resulted in a more significant increase in the cluster formation and hence the hardness of the alloy. This undefined precursor phase then acted as nuclei for the formation of precipitates after a long-time aging treatment. Therefore, two intermetallic precipitates, a spherical phase $\mathrm{Ni}_{16} \mathrm{Si}_{7} \mathrm{Ti}_{6}(\mathrm{G}-$ phase) and a rod-shaped $\eta$ - $\mathrm{Ni}_{3}(\mathrm{Ti}, \mathrm{Al})$ phase were generated during aging either simultaneously or separately depending on the chemical composition of alloys [78][84]. Both of the two precipitates were demonstrated to be correlated to the precipitation hardening. Despite the strong increase in strength at the early aging stage, the alloy suffered from severe intergranular embrittlement (Figure 2-1). It is 
speculated that the higher number density of undefined precursor precipitates was correlated to the embrittlement since neither precipitation nor segregation on the prior austenite grain boundaries was found at this stage. The embrittlement phenomenon was improved after prolonged aging due to the evolution of the precipitates and the reversion of austenite.

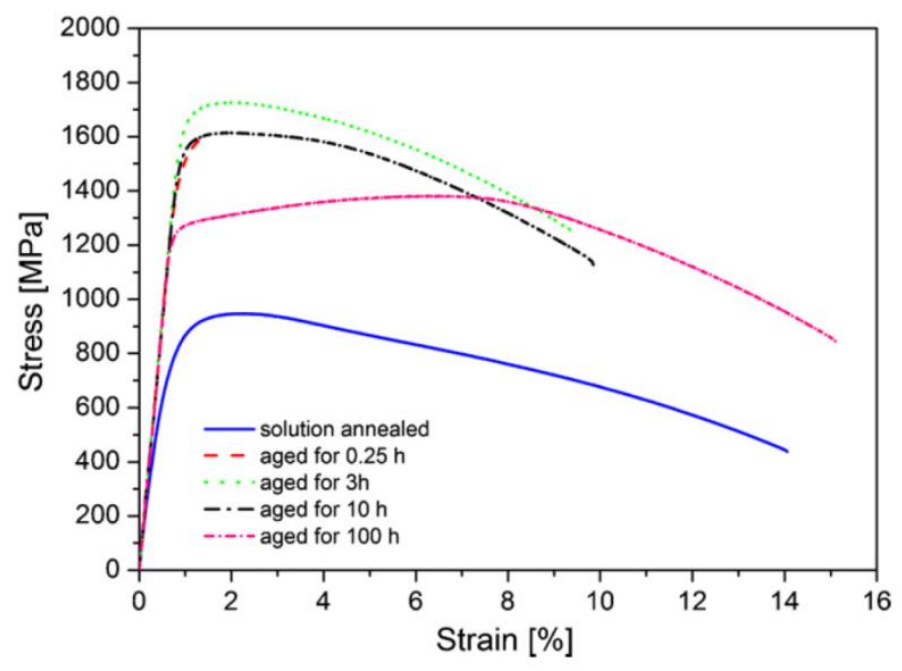

Figure 2-1 Stress-strain curves of samples with different heat treatment conditions in Ticontaining alloy [84].

\subsubsection{3 $\mathrm{L2}_{1}$-ordered $\mathrm{Ni}_{2} \mathrm{TiAl}$ type precipitates in $\mathrm{Fe}$-Cr-Ni-Al-Ti steels}

In contrast to the co-precipitation of $\mathrm{Ni}_{3} \mathrm{Ti}$ and $\mathrm{NiAl}$ described in Section 2.2.3.2, some studies demonstrated that the addition of $\mathrm{Ti}$ to $\mathrm{Fe}-\mathrm{Cr}-\mathrm{Ni}-\mathrm{Al}$ steels led to a type of precipitates which had rarely been reported in Fe-based alloys [62][85]. The nanosized precipitates which were observed in the as-quenched state presented a cuboidal morphology with an aspect ratio of $\sim 2$ and an average width of $15 \mathrm{~nm}$. The structure was determined as $\mathrm{L} 2{ }_{1}$-ordered and was fully coherent with the $\alpha$-Fe matrix (Figure 2-2(a) and (b) [85]). The precipitation of $\mathrm{L} 2{ }_{1}$-ordered $\mathrm{Ni}_{2} \mathrm{TiAl}$ phase from a supersaturated B2 TiNi matrix has been reported in several Ni-Ti-Al shape-memory alloys [86][87][88][89][90], but it was seldom observed in Fe alloys. During the further aging, the precipitates grew to an elongated shape with an average width of $40 \mathrm{~nm}$ and decomposed into a substructure with a network of $\mathrm{B} 2$-ordered $\mathrm{NiAl}$ zones 
in $\mathrm{L} 22_{1}$-ordered $\mathrm{Ni}_{2} \mathrm{TiAl}$ precipitates as shown in Figure 2-2(c). The $\mathrm{L} 2{ }_{1} / \mathrm{B} 2$ twophase system, with ordered structures based on a bcc lattice is analogous to the classical $\gamma / \gamma$, system with an fcc lattice in Ni-based superalloys [89][90]. Evidence showed that $\mathrm{Ni}_{2} \mathrm{TiAl}$ phase exhibited a better creep resistance than NiAl phase. In most creep-resistant form, the creep strength of $\mathrm{L}_{2}{ }_{1}-\mathrm{Ni}_{2} \mathrm{TiAl}$ between 1026 and 1273 $\mathrm{K}$ was about three times that of NiAl [91]. Further investigation revealed that the best creep properties in Ni-Al-Ti alloys was obtained by a two-phase microstructure of $\mathrm{NiAl}$ phase embedded in $\mathrm{Ni}_{2} \mathrm{TiAl}$ matrix [85]. Therefore, the formation of a similar two-phase precipitate which is composed of $\mathrm{B} 2$-ordered $\mathrm{NiAl}$ and $\mathrm{L}_{2}$ ordered $\mathrm{Ni}_{2} \mathrm{TiAl}$ is a potential strategy to optimize the mechanical properties of precipitation-strengthened ferritic alloys.
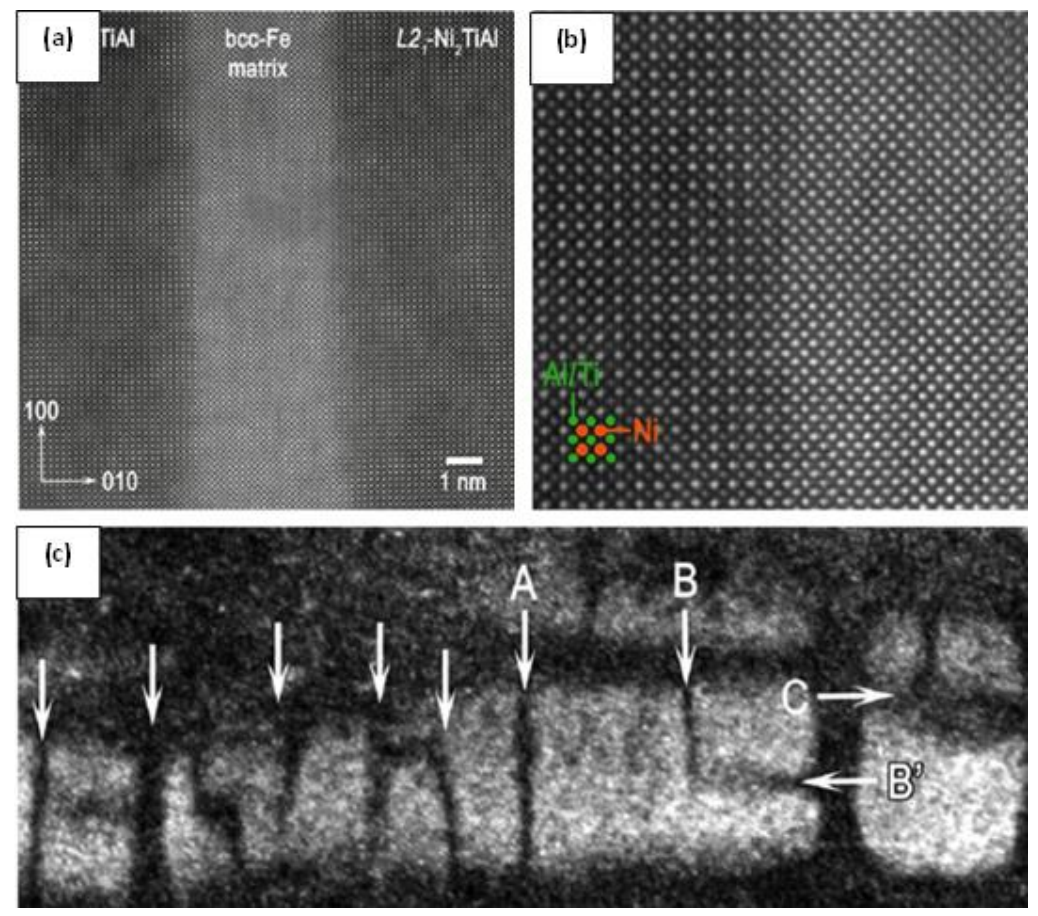

Figure 2-2 (a) An atomic resolution high-angle annular dark-field (HAADF)-scanning transmission electron microscope (STEM) image along the [001 $]_{\mathrm{bcc}-\mathrm{Fe}}$ zone axis; (b) magnified HAADF-STEM image of the precipitate/matrix interface in (a); (c) Dark-field image of the precipitates using the unique $1 \overline{1} \overline{1}$ reflection of $\mathrm{L}_{1}$-ordered $\mathrm{Ni}_{2} \mathrm{TiAl}$ phase where the $\mathrm{B} 2$-ordered $\mathrm{NiAl}$ zones are indicated by arrows [85]. 


\subsubsection{Effect of Mn on the precipitation in Fe-Ni-Mn-Ti-Al steels}

As discussed in Section 2.2.1, efforts of partial substitution of Ni by lean element such as Mn, which provides similar effects upon the austenite-martensite transformation, have led to the development of Fe-Ni-Mn-Ti-Al alloys. The increase of $\mathrm{Mn}$ content at the expense of $\mathrm{Ni}$ content is correlated to a considerable acceleration of the age hardening. A significant increase of hardness by $\sim 200 \mathrm{VHN}$ within $5 \mathrm{~s}$ was reported in an Fe-20Ni-1.8Mn-1.5Ti-0.59Al alloy aged at $550{ }^{\circ} \mathrm{C}$ and the yield strength increased by $\sim 215 \mathrm{MPa}$ to above $900 \mathrm{MPa}$ [48]. Initially the coclustering involving the $\mathrm{Ti}+\mathrm{Al}$ and $\mathrm{Mn}+\mathrm{Fe}$ was observed before the formation of plate-shaped $(\mathrm{Ni}, \mathrm{Fe})_{3} \mathrm{Ti}$ precipitates and spheroidal $(\mathrm{Ni}, \mathrm{Fe})_{3}(\mathrm{Al}, \mathrm{Mn})$ precipitates after aging for $\sim 60$ seconds [46]. Out of these four alloying elements, Ti possesses the highest diffusion rate followed by $\mathrm{Mn}$ and $\mathrm{Al}$, whereas $\mathrm{Ni}$ has the slowest rate. Therefore, the Ti-rich clusters normally acted as nuclei for the subsequent formation of $\eta-\mathrm{N}_{\mathrm{i} 3} \mathrm{Ti}$ precipitates [46]. On the other hand, the $(\mathrm{Ni}, \mathrm{Fe})_{3}(\mathrm{Al}, \mathrm{Mn})$ precipitates were found predominantly at two sites: homogeneously within the matrix or the periphery of plate-shaped $(\mathrm{Ni}, \mathrm{Fe})_{3} \mathrm{Ti}$ particles which indicated the segregation of $\mathrm{Al}$ and $\mathrm{Mn}$ at the interface between the $(\mathrm{Ni}, \mathrm{Fe})_{3} \mathrm{Ti}$ phase and surrounding matrix (Figure 2-3). This segregation of $\mathrm{Al}+\mathrm{Mn}$ atoms was attributed to the high thermodynamic affinity between $\mathrm{Al}$ and $\mathrm{Mn}$ [92][93][94] and the tendency of Mn atoms in steels to segregate on the interfaces [95][96]. 
(a)

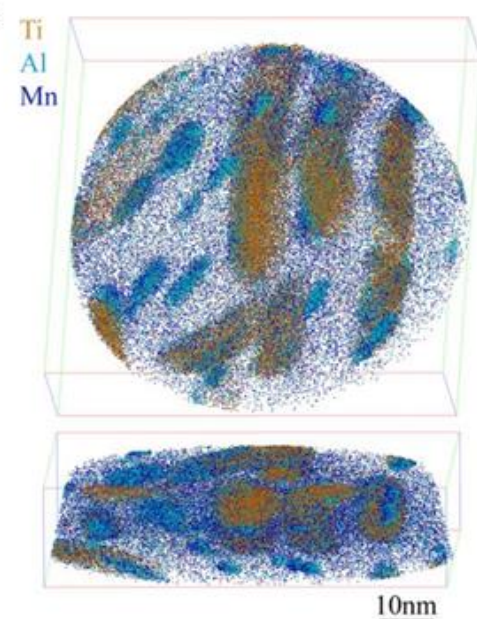

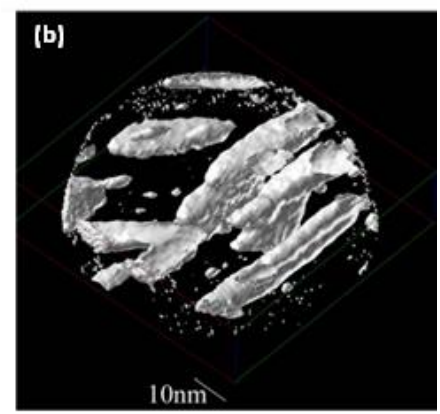

(b)

$10 \mathrm{~nm}$

Figure 2-3 (a) Three dimensional atom probe tomographic reconstructions of rod-shaped precipitates after treated for 3600 seconds and (b) corresponding $\mathrm{Ni}+\mathrm{Ti}$ isoconcentration surfaces [46].

\subsubsection{Cu-rich precipitation strengthened steels}

As $\mathrm{Cu}$ has limited solubility in $\mathrm{Fe}$ [97][98], $\mathrm{Cu}$ precipitates can serve as homogeneous nucleation sites for other precipitates [39][99]. It is also demonstrated that $\mathrm{Cu}$ accelerates the nucleation process due to a reduction in the activation energy when $\mathrm{Cu}$ is incorporated into precipitates [38][39]. Therefore, nano-sized $\mathrm{Cu}$ precipitates are extensively utilized in the development of precipitation-strengthened steels, and numerous studies have been carried out to study the hardening effect by $\mathrm{Cu}$ and $\mathrm{Cu}$-rich precipitates [38][39][40][45][97][99][100][101][102][103][104].

Investigations on the $\mathrm{Cu}$-containing steels revealed that $\mathrm{Cu}$ precipitates had a wide size range from $1 \mathrm{~nm}$ to $30 \mathrm{~nm}$ during aging [105][106]. Precipitation sequence was initiated with the rapid formation of metastable bcc copper phase which exhibited a spherical shape due to the coherency with bcc matrix [107]. The maximum hardness was achieved before $\mathrm{Cu}$ precipitates grew to the critical diameter of $\sim 5 \mathrm{~nm}$ after which the precipitates gradually lost the coherency and transformed to an intermediate 9R structure [106][107]. The precipitate/matrix orientation relationship was proposed as $(11 \overline{4})_{9 \mathrm{R}-\mathrm{Cu}}\left\|(011)_{\alpha-\mathrm{Fe}},[\overline{1} 10]_{9 \mathrm{R}-\mathrm{Cu}}\right\|[1 \overline{1} 1]_{\alpha-\mathrm{Fe}}[45][108]$. The twinned $9 \mathrm{R}$ 
precipitates maintained a spherical shape before coarsening to $\sim 17 \mathrm{~nm}$ [105]. Then a more stable $3 \mathrm{R}$ structure followed by an equilibrium fcc structure were observed in $\mathrm{Cu}$ precipitates during further aging. The rod-like fcc $\mathrm{Cu}$ precipitates regained the untwinned structure and were aligned along the Kurdjumov-Sachs orientation relationship $\quad\left((111)_{\mathrm{fcc}-\mathrm{Cu}}\left\|(110)_{\alpha-\mathrm{Fe}}, \quad[110]_{\mathrm{fcc}-\mathrm{Cu}}\right\|[111]_{\alpha-\mathrm{Fe}}\right)$ with the bcc matrix [100][102][105].

Despite of the advantages discussed above, steels strengthened by $\mathrm{Cu}$ precipitates suffered from low hardness and rapid precipitate coarsening during annealing. Therefore, minor additions of $\mathrm{Ni}, \mathrm{Ti}, \mathrm{Al}$ and $\mathrm{Mn}$ are introduced to develop the coprecipitation strategy so as to ultimately enhance the strengthening effect of $\mathrm{Cu}$ precipitates [38][40][102][109][110]. Two types of intermetallic precipitates, B2ordered $\mathrm{NiAl}$ and $\eta-\mathrm{Ni}_{3}(\mathrm{Ti}, \mathrm{Al})$, were generated into $\mathrm{Cu}$-containing maraging steels [38][39][40][45][102][103]. Experimental evidence revealed that the addition of $\mathrm{Cu}$ accelerated the nucleation of the two precipitates and in turn, the two phases restrained the coarsening of $\mathrm{Cu}$ precipitates.

It is worth discussing the different effects of $\mathrm{Cu}$ on the nucleation of the two precipitates. In the case of $\mathrm{B} 2$-ordered $\mathrm{NiAl}$ phase, approximately 10 at.\% $\mathrm{Cu}$ was detected within the $\mathrm{Ni}+\mathrm{Al}$ clusters in the as-quenched state [39] and this level remained constant during the following aging treatment. It is commonly believed that the incorporation of $\mathrm{Cu}$ reduced the nucleation energy by reducing the lattice misfit and thus promoted the precipitation of $\mathrm{NiAl}$ [38][39]. Moreover, $\mathrm{Cu}$-rich $\mathrm{NiAl}$ precipitates exhibited a stability in both the size and morphology compared to the $\mathrm{NiAl}$ precipitates in $\mathrm{Cu}$-free $\mathrm{Fe}-\mathrm{Ni}-\mathrm{Al}$ alloy [40]. In view of the rapid coarsening of both $\mathrm{Cu}$ precipitates and $\mathrm{NiAl}$ precipitates when present separately, it is supposed that there is an interaction effect between $\mathrm{Cu}$ and $\mathrm{NiAl}$ phase in terms of the resistance to coarsening.

On the other hand, a different $\mathrm{Cu}$-rich precipitation behaviour was reported in other Fe-Ni-Al alloys [45][102][103]. The initial precipitation was similar as described above: short-range ordering occurred before aging followed by the formation of B2ordered Ni-Al-Cu precipitates at the beginning of aging as shown in Figure 2-4(a) [45]. Then a decomposition of the primary precipitates led to a kind of core/shell 
precipitates with $\mathrm{Cu}$-rich core surrounded by a periphery enriched in $\mathrm{Ni}$ and $\mathrm{Al}$ (Figure 2-4(b) and (c)). The thermally stable B2-ordered shells hindered the diffusion-controlled growth of $\mathrm{Cu}$-rich core, and more importantly, reduced the misfit between the fcc $\mathrm{Cu}$-rich core and the bcc matrix, Figure 2-4(d) and (e) [45][102]. The low interfacial energy together with the atomic ordering of B2ordered shells allowed the precipitates to grow moderately without sacrificing the high-density feature during further aging, and moreover provided a complex obstacle for dislocation motion [103].
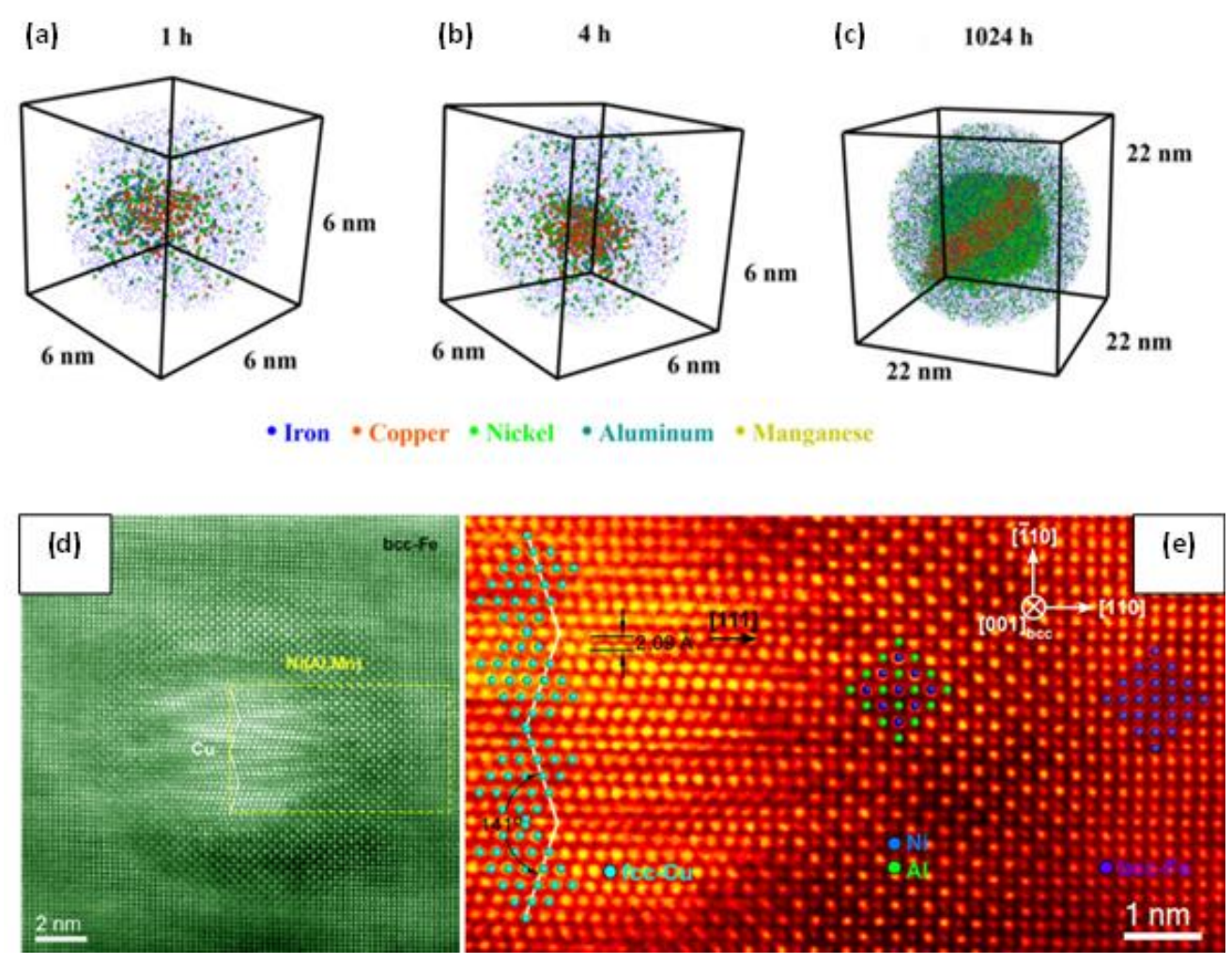

Figure 2-4 Atomic distribution of representative precipitates in the (a) $1 \mathrm{~h}$, (b) $4 \mathrm{~h}$ and (c) $1024 \mathrm{~h}$ aging stages [102]; (d) HAADF image of the core/shell precipitates and (e) Enlarged HAADF image of yellow rectangle part in (d) [45].

In terms of the $\mathrm{Ni}_{3} \mathrm{Ti}$-strengthened steels, as the solubility of $\mathrm{Cu}$ in $\mathrm{Ni}_{3} \mathrm{Ti}$ phase is much lower than that in $\mathrm{NiAl}$ phase, the independent $\mathrm{Cu}$-rich clusters were usually observed in the beginning of aging and acted as nucleation sites for $\mathrm{Ni}_{3}(\mathrm{Ti}, \mathrm{Al})$ phase. The precipitation of $\mathrm{Ni}_{3}(\mathrm{Ti}, \mathrm{Al})$ occurred mainly adjacent to the $\mathrm{Cu}$-rich clusters [39]. 
Other studies reported that the formation of $\eta-\mathrm{Ni}_{3} \mathrm{Ti}$ in $\mathrm{Cu}$-containing maraging steels was restrained when $\mathrm{NiAl}$ precipitates were also present, as $\mathrm{Cu}$ could only be incorporated in $\mathrm{NiAl}$ phase and thereby, to a larger extent, accelerating the formation of $\mathrm{NiAl}$ phase in the initial aging stage[38].

\subsubsection{The Mn embrittlement in Fe-Ni-Mn and Fe-Mn alloys}

As mentioned in Section 2.2.1, attempts have been made to develop cheaper alternatives to the conventional Ni-based maraging steels. Experimental studies on a series of Fe-Ni-Mn steels revealed that this type of steels exhibited a remarkable maraging strengthening owing to the formation of fine f.c.t $\theta-\mathrm{NiMn}$ phase [111][112]. In some cases, the addition of $\mathrm{Ti}$ to $\mathrm{Fe}-\mathrm{Ni}-\mathrm{Mn}$ alloys was found to generate very fine and thermally stable $\mathrm{Ni}_{3} \mathrm{Ti}$ precipitates [33]. Other phases such as $\mathrm{MnNi}$, fcc $\mathrm{Ni}_{3} \mathrm{Mn}$ and ordering were also considered as possible precipitationstrengthening contributor [113][114]. Therefore, this grade of steels used to be regarded as a promising alternative to conventional maraging steels.

Unfortunately, serious grain-boundary embrittlement was observed to occur at the very beginning of aging treatment in Fe-Ni-Mn steels [95][115][116][117]. A lot of researches were carried out on the source of grain boundary embrittlement and some possibilities have been proposed. It is generally accepted that the grain boundaries act not only as barriers against dislocation motion but also as zones where interface segregation may occur. More specifically, Raabe et al. [50] summarized the conceivable pathways by which the solute segregation could behave: (i) the segregation might increase the coherence and preferential bonding at the interface (grain boundary strengthening); (ii) the reverse could lead to a further loss of coherence and unfavourably directed bonding at the interface (grain boundary weakening); (iii) phase transformation might occur at the grain boundaries (grain boundary phase transformation); (iv) the formation of one or more second phases at the decorated interface could be initiated (grain boundary precipitation); (vi) discontinuous precipitation might be promoted.

Squires and Wilson [95] first stated that the intergranular fracture observed in an Fe$12 \mathrm{Ni}-6 \mathrm{Mn}$ alloy was associated with the segregation of embrittling elements like $\mathrm{Mn}$ 
and $\mathrm{P}$ to prior austenite grain boundaries. A later study on a similar alloy complementally reported that the $\mathrm{Ni}$ and $\mathrm{N}$ were also found at the fractured grain boundaries [115]. Heo and Lee [117] tracked the segregation and desegregation of $\mathrm{Mn}$ at the prior austenite grain boundaries on the fracture surfaces of an $\mathrm{Fe}-8 \mathrm{Mn}-7 \mathrm{Ni}$ alloy in the embrittled condition. They pointed out that the embrittlement and deembrittlement of the alloy was directly correlated to the segregation and desegregation of $\mathrm{Mn}$, but they also suggested that considering the low enrichment level of Mn, such serious embrittlement was not likely to be solely attributed to the Mn segregation alone [92].

In contrast, Suto and Murakami argued that the slight segregation of $\mathrm{Mn}$ and $\mathrm{Ni}$ at grain boundaries detected would not result in the transition to brittle state [116]. Raabe and his colleagues [50] also hold an opposite opinion about the correlation between Mn segregation at grain boundary and mechanical behaviours. They believed that the embrittlement was associated with the susceptible crack penetration owing to the low mutual misorientation between adjacent lath boundaries [50]. Unfortunately, as the predominant interfaces in quenched martensite, a large amount of lath boundaries existed. However, given that the variation of bulk grain free energy was negligible, the primary thermodynamic driving force for solute segregation at interfaces was to minimize the interface free energy. As the decrease of the interfacial free energy reduced the driving force for grain growth, materials with reduced grain boundary energy were considered to have a finer and more stable grain size. Therefore, they proposed that the solute segregation at grain boundaries contributes to both strength and toughness by refining grain size, if the specific type of segregation does not lead to grain boundary embrittlement.

On the other hand, other studies suggested the intergranular embrittlement in Fe-Ni$\mathrm{Mn}$ system as the result of the interaction of mobile dislocation with precipitates at grain boundaries. These researchers insisted that the precipitation on grain boundary was the main source of grain boundary failure in Fe-Ni-Mn maraging steels. Lee et al. [118] found $\theta-\mathrm{NiMn}$ intermetallic precipitates at the grain boundaries in an $\mathrm{Fe}$ $10 \mathrm{Ni}-5 \mathrm{Mn}$ alloy when aging at $753 \mathrm{~K}$ for short time and this type of precipitate was initially suspected to be responsible for the embrittlement. Later studies conducted by Nedjad et al. [119][120] on Fe-10Ni-7Mn maraging steels confirmed the 
correlation between the intergranular failure and precipitation reactions at grain boundaries. They further pointed out that the formation of coarse precipitates at grain boundaries resulted in the soft solute-depleted precipitate-free zones at grain boundaries. Then the large strain localization resulting from the grain boundary weakening triggered the microcracks initiation under negligible macroscopic strains. Alternatively, Mun et al. [113] observed the precipitation of austenite particles along grain boundaries in an Fe-7Ni-8Mn steel and it was assumed that the austenite-ferrite interface boosted the intergranular fracture. However, Wilson and his colleagues [37][121] argued that the driving force for Mn segregation to prior austenite grain boundaries was indeed to form precipitates and reverted austenite, but the primary factor of grain boundary embrittlement was still the segregation of Mn rather than precipitation or austenite reversion.

Binary Fe-Mn alloys in the range of 4-10 wt.\% Mn, which possess a soft but heavily dislocated lath martensite after quenching, generally exhibit similar microstructural features to Fe-Ni-Mn maraging steels. Studies on their mechanical behaviours revealed that Fe-Mn alloys suffered from brittleness as well [121][122]. After cooling from the austenite phase field, brittle fracture occurred mainly by cleavage. While tempering at $250-500{ }^{\circ} \mathrm{C}$ increased the DBTT and the failure mode below DBTT was intergranular fracture. The maximum embrittlement was reported at $450{ }^{\circ} \mathrm{C}$ [121]. Elevated temperature, e.g. $600{ }^{\circ} \mathrm{C}$, has shown to overcome the embrittlement due to a faster kinetics to form ductile austenite [50][28].

Therefore, the embrittlement is of key importance in the mechanical behaviour of Fe-Ni-Mn and Fe-Mn alloys. To understand the embrittlement mechanism can provide not only an insight into the alloying element interaction in these alloys during thermal treatment but also pathways to improve their possible commercial exploitation. However, to the author's best knowledge, the mechanism of intergranular fracture in Fe-Ni-Mn and Fe-Mn systems is still not clear yet and remains the subject of debate in the literature. 


\subsection{Evolution of precipitates}

Generally, the precipitation process is generally described as successive nucleation and growth followed by coarsening. In this section, the discussion about precipitate evolution will mainly focus on the growth of nuclei and then on the coarsening.

\subsubsection{Critical size in precipitation}

The real precipitation process is always complicated. It is worth introducing several critical sizes during the evolution of precipitation before discussing the precipitation mechanism. The critical precipitate radius $\left(r_{c}\right)$ below which the precipitate will dissolve is usually determined by [29]:

$$
r_{c}=2 c_{\alpha} \Gamma /\left(c_{0}-c_{\alpha}\right)
$$

where $c_{\alpha}$ is the solute concentration in the matrix phase during aging, $c_{0}$ is the solute concentration in the matrix before aging. The capillarity constant $\Gamma$ is described as [29]:

$$
\Gamma=\frac{\sigma_{\alpha / \beta} N_{A} \Omega_{\beta}\left(1-c_{\alpha}\right)}{R T\left(c_{\beta}-c_{\alpha}\right)}
$$

where $\sigma_{\alpha / \beta}$ is the interfacial energy per unit area between the precipitate and matrix, $N_{A}$ is Avogadro's number, $\Omega_{\beta}$ is the atomic volume of precipitates, $c_{\beta}$ is the solute concentration in the precipitate, $R$ is the idea gas constant and $T$ is the absolute temperature.

The first critical precipitate radius $r_{c 1}$ is defined to distinguish the growth mechanism of precipitates above which the interfacial-limited growth is taken over by the diffusion-limited growth. After that, further precipitation results in another critical precipitate radius $r_{c 5}$ above which the diffusion-limited coarsening occurs. In Section 2.3.2, the detail about the growth and coarsening of precipitates will be discussed.

As the interfacial free energy $\sigma_{\alpha / \beta}$ (Equation (2.2)) varies depending on the nature of coherency, two more critical sizes, $r_{c 0}$ and $r_{c 3}$, are introduced. $r_{c 0}$ is the critical 
radius below which precipitates will dissolve when the interface is coherent and $r_{c 3}$ is the critical radius below which precipitates will dissolve when the interface is incoherent.

Another two critical sizes relating to the precipitation strengthening are also taken into consideration. $r_{c 2}$ stands for the critical radius above which the coherency strengthening starts to decrease, whereas $r_{c 4}$ indicates the critical radius above which the interaction between dislocation and the precipitate transforms from shear-cutting mechanism to looping mechanism.

In order to better understand the precipitation process, efforts have been made to sort out the order of these critical sizes. It is not difficult to understand that $r_{c 1}<r_{c 5}$ and $r_{c 0}<r_{c 2}<r_{c 3}$. As $r_{c 0}$ and $r_{c 1}$ are very small, they are usually considered as zero [29]. In most cases, the Orowan looping occurs when precipitates are incoherent with the matrix, thus we can obtain $r_{c 3}<r_{c 4}$ (dislocation looping may also occur when the coherent precipitate is too large or strong to be cut through). Thus what is uncertain is the relationship of $r_{c 5}$ with the other three critical sizes $r_{c 2}, r_{c 3}$ and $r_{c 4}$ and this will be further discussed in Section 2.3.4.

\subsubsection{Growth and Coarsening of precipitates}

During the early stage of precipitation, the nucleus is surrounded by a supersaturated matrix with a solute concentration gradient which provides the driving force for solute diffusion and promotes the precipitate growth. There are two factors which are considered to affect the growth rate: the interface reaction and the lattice diffusion [123]. When the average size of precipitates is below $r_{c 1}$, as the distance of diffusion field is rather short, the interface reaction is the rate-controlling step. The precipitate size is proportional to the aging time: $\bar{r}=r_{c 0}+G_{0} t$ (Figure 2-5), where $G_{0}$ is the growth rate during the interface-limited growth. In the case of larger precipitates $\left(r>r_{c 1}\right)$, the driving force for lattice diffusion gradually decreases owing to the continuous depleting of solute atoms in the matrix, the diffusion becomes the ratecontrolling step [123][124]. The relationship between the precipitate size and growth time follows the equation [29][125]: 


$$
\bar{r}=\left(r_{c 1}^{2}+2 D \frac{c_{0}-c_{\alpha}^{e}}{c_{\beta}-c_{\alpha}^{e}}\left(t-t_{1}\right)\right)^{1 / 2}
$$

where $D$ is the diffusion coefficient in the matrix, $c_{0}$ is the initial solute concentration, $c_{\alpha}^{e}$ is the equilibrium solute concentration in the matrix, $c_{\beta}$ is the solute concentration in the precipitate and $t_{1}$ is the time the diffusion-limited growth starts.

At the end of the diffusion-limited growth period, Equation (2.3) cannot describe the dynamic evolution of precipitate size anymore which indicates that the diffusionlimited coarsening initiates. It is difficult to accurately distinguish the diffusionlimited growth and diffusion-limited coarsening. Generally, the diffusion-limited growth process is defined as a stage when the solute is from the surrounding matrix; whereas the solute for diffusion-limited coarsening is from the dissolving of smaller precipitates. According to the Gibbs-Thomson equation, the solubility of smaller precipitates which possess a larger ratio of surface area to volume is higher than that of larger precipitates. This size-dependent solubility results in a further sizedependent driving force for coarsening. Based on the Gibbs-Thomson equation, the growth rate is positive for large precipitates with $\bar{c}<c_{R}$ and negative for small precipitates with $\bar{c}>c_{R}$, namely, larger precipitates grow at the expense of smaller precipitates which dissolve back into the matrix. Therefore, the coarsening process is featured by the decrease of number density and the broadening of size distribution. But in reality, coarsening may take place simultaneously with the growth process or even in the nucleation stage if the initial solid solution supersaturates [124]. In addition, due to the increase in the distances of diffusion field, the size increment rate in the coarsening stage is slower than that in the growth stage (Figure 2-5). The precipitate size in most coarsening process obeys the LSW (Lifshitz and Slyozov [126] and Wagner [127]) theory which suggests the time exponent is $1 / 3$ (Figure 2-5). 


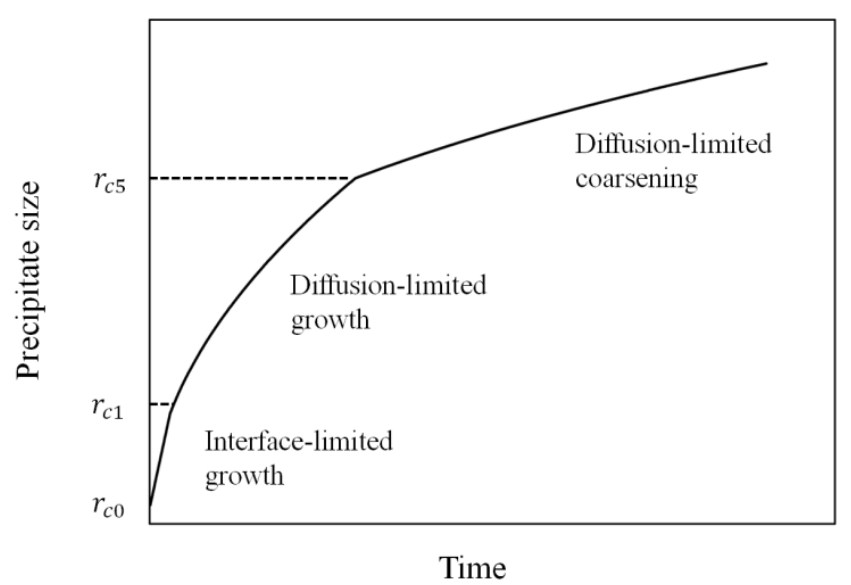

Figure 2-5 The evolution of precipitate size during aging in terms of precipitation kinetics, after [29].

\subsubsection{Precipitate coarsening theory}

Although it is difficult to describe the coarsening of randomly dispersed precipitate, Lifshitz and Slyozov [126] and Wagner [127] developed a theory attempting to interpret the diffusion-limited coarsening. The theory which is referred to as LSW theory has later achieved great success in the experiment application. Certain assumptions are made to confine the application [124][128]:

1. Dilute solution theory applies and the linearized version of the GibbsThomson equation is valid.

2. Diffusion fields of precipitates do not overlap and the particles only interact with the matrix, thereby limiting the precipitate volume fraction to zero.

3. Coarsening takes place in a stress-free matrix.

4. Precipitates possess a spherical morphology.

5. The composition of precipitates is the same as given by the equilibrium phase diagram.

Based on the above assumptions, the LSW theory provides three equations to describe the diffusion-limited coarsening. The first equation predicts the increase of the average precipitate radius, $\bar{r}$, with respect to the coarsening time according to: 


$$
\bar{r}^{3}-r_{c 5}^{3}=\frac{8 D V_{\beta} \sigma_{\alpha / \beta}}{9\left(c_{\beta}^{e}-c_{\alpha}^{e}\right)^{2} G_{\alpha}^{\prime \prime}} t
$$

where $\mathrm{D}$ is the diffusion constant, $V_{\beta}$ is the molar volume of $\beta$ phase, $\sigma_{\alpha / \beta}$ is the interfacial energy per unit area between the precipitate and matrix, $c_{\alpha}^{e}$ and $c_{\beta}^{e}$ are the equilibrium solute concentration in the matrix and precipitates respectively, $G_{\alpha}^{\prime \prime}$ is the second derivative of the molar Helmholtz energy of the $\alpha$ phase.

The second equation reveals that the decrease in the precipitate number density, $N_{V}$, follows:

$$
\mathrm{N}_{\mathrm{V}} \cong \frac{3 \mathrm{f}_{\mathrm{p}}}{4 \pi} \frac{1}{\mathrm{~K}_{\mathrm{R}} \mathrm{f}_{3}} \mathrm{t}^{-1}
$$

where $f_{p}$ is the volume fraction of precipitate, $K_{R}$ is the coarsening rate constant in Equation (2.4) and $f_{3}$ is the third moment of the time-independent precipitate size distribution function $f(r / \bar{r})$ when $V_{p} \rightarrow 0$ (will be discussed later).

The relationship between the solute concentration within the matrix, $C_{i}^{\alpha}$, and coarsening time is derived:

$$
\Delta c=\frac{9\left(c_{\beta}^{e}-c_{\alpha}^{e}\right)}{4 D} K_{R}^{2 / 3} t^{-1 / 3}
$$

Numerous experimental studies on two-phase alloys have been carried out to examine the LSW coarsening theory. Advanced techniques such as CTEM[76][129], HREM, STEM and HAADF-STEM [45][130] provide direct observations of the local structural characteristics. In some studies, small-angle X-ray scattering (SAXS) and small-angle neutron scattering (SANS) approaches are applied for the quantitative analyses of average precipitate size, interparticle distance and volume fraction based on huge numbers of precipitates [97][131][132]. Moreover, SAXS can also provide information about the chemical heterogeneity of precipitates [133][134]. Recently, state-of-the-art APT offers not only an accurate quantitative measurement of precipitates [130][135] but also a three dimensional observation on the atomic distribution within the material. If combined with the complementary studies on the resistivity and hardness evolution, global information about the coarsening of precipitates can be obtained. 
Most of these experimental studies revealed that the experimental plot of $\bar{r}^{3}$ or $N_{V}^{-1}$ vs. $t$ roughly fitted the straight lines given by LSW theory. However, experimental results also indicates that the shapes of size distribution function are much broader than that of the theoretical $f(r / \bar{r})$. This deviation has later been demonstrated to be associated with the non-zero volume fraction of precipitate in reality. Therefore, many studies have been carried out to modify the LSW theory for better applications in cases where the volume fraction of precipitate has to be taken into consideration. The major challenge of this work is to determine the effects of interparticle diffusional interactions on the coarsening behaviour of a precipitate with a specific size. Modified theories [136][137][138][139] are all in agreement that the exponents of the Equations (2.4), (2.5) and (2.6) and the time-independence of precipitate size distribution will not change when the volume fraction of precipitates is taken into consideration. However, the values of $K_{R}$ and $K_{N}$ along with the shape of size distribution function will change. According to the modified theories, with the increase of precipitated volume fraction, the average distance of diffusion field become shorter and thus the concentration gradients will be larger, thereby resulting in the increase of the coarsening rate. Besides, the local diffusional interactions give rise to spatial correlations between adjacent precipitates which further leads to the broadening and symmetry of size distribution function.

\subsubsection{The effect of precipitate size on precipitation strengthening}

It is easy to get confused when discussing the relationship of coherency, coarsening and strengthening mechanism. It is generally thought that in the under-aged stage the growth of coherent precipitates leads to an increase in the strength with shear-cutting mechanism. While in the overaged stage, the coarsening of precipitates results in the incoherency and thus activates the looping softening mechanism. Although most precipitation-strengthened alloys obey this rule, there are several concepts which are confusing.

First, the term 'strengthening' is usually utilized to describe the strength increase in the underaged stage and 'softening' means the decrease of strength during the 
overaged stage. But it is worth noting that, the strength at both of the two stages is higher than that before aging, therefore both of two terms are actually defined as strengthening mechanisms.

Secondly, although the softening during the overaged period is generally considered to result from lose of the coherency, a number of studies have shown that the extremely large coherent precipitate and the modulus difference between the precipitate and matrix can also lead to the decrease of strength. In the case of large precipitates, considerable flexing of dislocation occurs owing to the increase of the interparticle spacing (assuming the volume fraction of precipitate is constant), thereby resulting in the coherency softening. Namely, the coherency softening may occur before the lose of coherency. Another possible is the modulus difference which is suggested to result in the softening as well, but so far there is no experimental evidence to support this viewpoint yet [29].

Thirdly, the strengthening mechanism is only associated with the precipitate size regardless of how the precipitate approaches the critical size, by growth or coarsening. It means that $r_{c 2}$ and $r_{c 4}$ are irrelevant to $r_{c 5}$. But according to Sha and Guo's conclusion [29], the value of $r_{c 2}$ should be somewhere between $r_{c 1}$ and $r_{c 5}$.

Based on the discussion, a more comprehensive precipitation-strengthening process is comprised of three stages: when the precipitate is small, coherency strengthening takes effective by cutting mechanism, the strengthening effect increases with the precipitate size; with the growth of precipitate size, the stress required to cut through precipitate is so high that Orowan looping mechanism takes place. Orowan strengthening is inversely related to the interpartical spacing, so initially the precipitate growth which reduces the interpartical spacing lead to the Orowan strengthening. The following coarsening of precipitate featured with the decrease of number density and thus the increase of interpartical spacing results in the Orowan Softening mechanism. On the other hand, the structure of the interface is also very important. For example, if the precipitates are small but have incoherent interface, the looping mechanism take place. Conversely, if the precipitates have a large size but coherent interface and a small misfit strain, the shearing will be the dominant mechanism. 
It is worth emphasizing that apart from the coherency mechanism and modulus mechanism, there are more strengthening mechanisms, such as chemical (softening), stacking-fault (strengthening), order (strengthening), etc. As the effects of these mechanisms on the strengthening are irrelevant to the precipitate size, they are not taken into consideration when discussing the relationship between the strengthening and precipitate size.

\subsection{Austenite reversion in maraging steels}

As previously stated, engineering steels suffer from the fact that the increase of strength generally corresponds to a decrease in ductility. This inverse strengthductility relationship largely limits their application as structural materials. In order to overcome this problem, dual-phase microstructure, such as hard martensite with ductile ferrite or austenite which can coordinate during deformation, is developed. Moreover, for steels containing austenite, the deformation-induced martensitic transformation of austenite under external load can provide additional strength and elongation. This type of steels has now found wide applications in the body frame sector of automotive manufacture. Their high strength-to-weight ratio, low yield-toultimate strength ratio, high initial work hardening rate and good formability make them particularly suitable for this application [140].

Austenite in steels is generally derived from two different processing technologies. The austenite phase retained after cooling from two-phase field is known as retained austenite and it has been investigated extensively in TRIP steels [141][142][143][144]. Austenite phase which is formed by a partial reversion from martensite when aging at two phase field but a lower temperature is defined as reverted austenite [145][146][147]. Compared to retained austenite, reverted austenite normally provides more significant microstructural synergy with martensite, as it creates thin compliant interlayers along both prior austenite grain boundaries and martensite lath boundaries.

The influence of reverted austenite on the mechanical properties of maraging steels has been investigated by many studies. Conventionally, the intermetallic precipitates in maraging steels are thought to be the main contributor to the strength 
enhancement upon aging [31][48][148][149][150][151]. However, it has been gradually recognized that reverted austenite formed in some maraging steels also has a pronounced effect on the mechanical properties [18][19][20][145][152][153]. It is proposed that [121]:

1. Austenite can act as a sink for impurities; in this case, reducing $\mathrm{N}$ and $\mathrm{P}$ embrittlement during heat treatment.

2. The ductile phases, $(\gamma+\varepsilon$ ( $\varepsilon$ martensite) $)$, may act as compliance layers or mechanical buffer regions impeding crack propagation otherwise prevalent along the $\{100\}$ planes of martensite laths, which has been considered to improve toughness and reduce DBTT [36].

3. Transformation of austenite to martensite may occur during deformation which also improves toughness.

\subsubsection{Influence of reverted austenite on mechanical properties}

Many researches have been carried out to study the effects of reverted austenite on mechanical properties in maraging steels. Vylezhnev et al. [153] proposed that the poor ductility at low temperatures in a $18 \mathrm{Ni}$ maraging steel resulted from the fracture of reverted austenite along martensite lath boundaries. Viswanathan et al. [19] noticed that the austenite reversion in a $18 \mathrm{Ni}$ maraging steel corresponded to the decrease in both yield strength and ultimate tensile strength but the increase in ductility at the initial overaged stage. Vijay et al. [54] attributed the high strength after long-term aging in a Co-free, high- $\mathrm{Ti} 18 \mathrm{Ni}$ maraging steel to the high coarsening resistance of precipitates and a small amount of reverted austenite, whereas in the case of a Co-containing, low-Ti $18 \mathrm{Ni}$ maraging steel, the high volume fraction of reverted austenite are considered to result in the loss of strength at long aging times. Overall, the debate on this issue has been chaotic for years. However, recent researches tend to hold the same opinion that the formation of reverted austenite can lead to the increase of strength and toughness in Co-free maraging steel. Nakagawa et al. [20] reported a significant improvement in the balance of strength and ductility during the overaged stage in $\mathrm{Cu}$-containing maraging steels, and they suggested this improvement is due to the increase in the amount of reverted austenite. 
Raabe et al. [18], reported a significant increase in both strength and ductility upon aging in a 9 wt.\% Mn maraging TRIP steel with the ultimate tensile strength increased by $25 \%$ (from $810 \mathrm{MPa}$ to $1 \mathrm{GPa}$ ) and total elongation increased by more than $150 \%$ (from $6 \%$ to $15 \%$ ), which is considered as a result of nano-precipitation and nano-scale austenite reversion.

As revealed by these researches, the mechanical properties is directly associated with the quantity of austenite. An appropriate quantity of reverted austenite can lead to a good balance between strength and toughness. Generally, the quantity of austenite is determined by the chemical composition, aging temperatures and durations [27][18]. In addition to the quantity, the stability of reverted austenite also plays a critical role in the mechanical properties of maraging steels. As the literature about the stability of reverted austenite is limited, retained austenite may be discussed alternatively in this study. In TRIP and Q\&P steels, retained austenite with proper stability to induce martensitic transformation is desirable. Retained austenite with low stability would transform at small strains and cannot improve the ductility; whereas extremely high stability does not lead to the TRIP effect and contributes little to ductility either. The stability of austenite is generally governed by the chemical composition $[154][34][155][156][157][158][159], \quad$ temperature $\quad[160][161], \quad$ size [154][34][158][159][162][163], morphology [164][165], adjacent microstructure [157][159][165] and crystallographic orientation relationship [166][167][168][169]. The following part will discuss reverted/retained austenite in terms of these factors.

\subsubsection{Composition}

The chemical driving force for martensitic transformation of austenite involving the most potential austenite stabilizing elements, carbon and manganese, is described as [170]:

$$
\begin{gathered}
\Delta G^{c h}=-7381.6+19296 X_{M n}+69447 X_{C}-38776 X_{M n} X_{C} \\
+\left(6.7821-33.45 X_{C}\right) T
\end{gathered}
$$

where $\Delta G^{c h}$ is the chemical driving force for martensitic transformation, $X_{M n}$ is the mole fraction of manganese, $X_{C}$ is the mole fraction of carbon and $T$ is the absolute temperature. As shown in Equation (2.7), the increase in the mole fraction of 
manganese and carbon leads to the increase of the driving force for martensitic transformation at specific temperature, namely, the addition of carbon and manganese is beneficial to the stability of austenite.

Similarly, Ni, as another efficient austenite stabilizer, contributes to the austenite stability as well; whereas elements which are defined as ferritic stabilizer, such as Ti and $\mathrm{Al}$, are demonstrated to decrease the austenite stability.

\subsubsection{Temperature}

The effect of temperature can be expressed by the equation [142]:

$$
\frac{\partial \Delta G^{c h}}{\partial T}=6.7821-33.45 X_{c}
$$

According to the Equation (2.8), a positive fluctuation in $\Delta G^{c h}$ is expected when a positive fluctuation in temperature occurs with a constant carbon content, i.e. austenite is more stable at higher temperature. This view point has been confirmed by tensile tests conducted at a range of temperatures which reveals the variation of austenite stability depending on temperatures [160][161].

\subsubsection{Size}

Yang and Bhadeshia described the relationship between austenite grain size and $M_{S}$ temperature as:

$$
M_{s}^{0}-T=\frac{1}{b} \ln \left[\frac{1}{V_{\gamma}}\left\{\exp \left(\frac{\ln \left(1-f_{M}\right)}{m}\right)-1\right\}+1\right]
$$

where the constant $b$ is 0.2689 for $\alpha^{\prime}$-martensite [162] and 0.19 for $\varepsilon$-martensite [26], $V_{\gamma}$ is the average grain volume of austenite, $f_{M}$ is the volume fraction of martensite, $m$ is the aspect ratio of the martensite ( $\alpha^{\prime}$-martensite: $0.05 ; \varepsilon$-martensite: 0.03 ), and $T$ is the temperature at which $f_{M}$ is measured. In this equation, $M_{S}^{0}-T$ becomes $M_{S}^{0}-M_{S}$ if $f_{M}$ is set to be the first detectable fraction of martensite, $V_{\gamma} \rightarrow \infty$ and so $M_{S} \rightarrow M_{S}^{0}$. According to their conclusion, the small size of austenite suppresses the $M_{S}$ temperature and therefore contributes to the stability of austenite. The size effect on the austenite stability in steels has been extensively investigated by experiment as 
well. In carbon TRIP steels, both experimental results and thermodynamic modelling have demonstrated that $M_{s}$ temperature decreases as the carbon concentration of retained austenite increases or the mean size of retained austenite decreases [161]. In Mn-containing TRIP steels, it is shown that the size effect of ultrafine austenite grain and the partitioning of $\mathrm{Mn}$ to austenite during intercritical annealing were the two primary reasons for the high mechanical stability of retained austenite [34].

In contrast, recent work by Wang et al. [171] reported that small austenite grains were less stable against the deformation-induced martensitic transformation in a 9 wt.\% Mn TRIP maraging steel. They suggested the deformation-induced martensitic transformation was suppressed by the mechanical twinning in larger austenite grains while in smaller austenite grains, mechanical twinning is less favoured. Other work [166] proposed that because the intrinsic strength of austenite is lower than that of the martensite matrix, a parabolic distribution of the von Mises stress exists in reverted austenite, namely the stress in the outer layer is higher than that in the centre. Besides, there is an inverse distribution of austenite stabilizing element concentrations in the reverted austenite grains. Hence, it is easier for the outer layer of reverted austenite to transform into martensite during the deformation. Generally, the fraction of the outer layer region is smaller in the reverted austenite with a larger dimension and so it is proposed that the larger reverted austenite is more stable than the smaller one.

\subsubsection{Morphology}

Reverted austenite, which is distinguished from retained austenite, exhibits different morphologies [146][147]. Evidence indicates that the morphologies are correlated to the alloy compositions and processing paths [147]. Reverted austenite in maraging steels generally appears either an elongated or granular shape. More specifically, reverted austenite is classified into three types, i.e. matrix austenite, lath-like austenite and recrystallized austenite [146]. Matrix austenite is defined as an austenite phase that either develops from retained austenite and thus has the same orientation, or grows at the prior austenite grain boundaries and forms a single austenite grain (Figure 2-6(a)). Lath-like austenite can be generated along and within the martensite laths, thereby generating a lamellar structure of alternate austenite 
laths and residual martensite laths (Figure 2-6(b)). Recrystallized austenite normally nucleates at high aging temperatures or after long aging times and it is characterized by a polygonal shape with low dislocation density, as shown in Figure 2-6(c). In addition, in high $\mathrm{Ni}$ alloyed and Ti-containing maraging steels, a type of Widmanstätten austenite was reported to become dominant when aging at high temperatures for a long time [52][172]. In terms of its effect on the austenite stability, the morphology does not directly affect the mechanical stability of austenite, it is actually the adjacent microstructure that determines the mechanical stability of reverted austenite and this will be discussed in the next section.
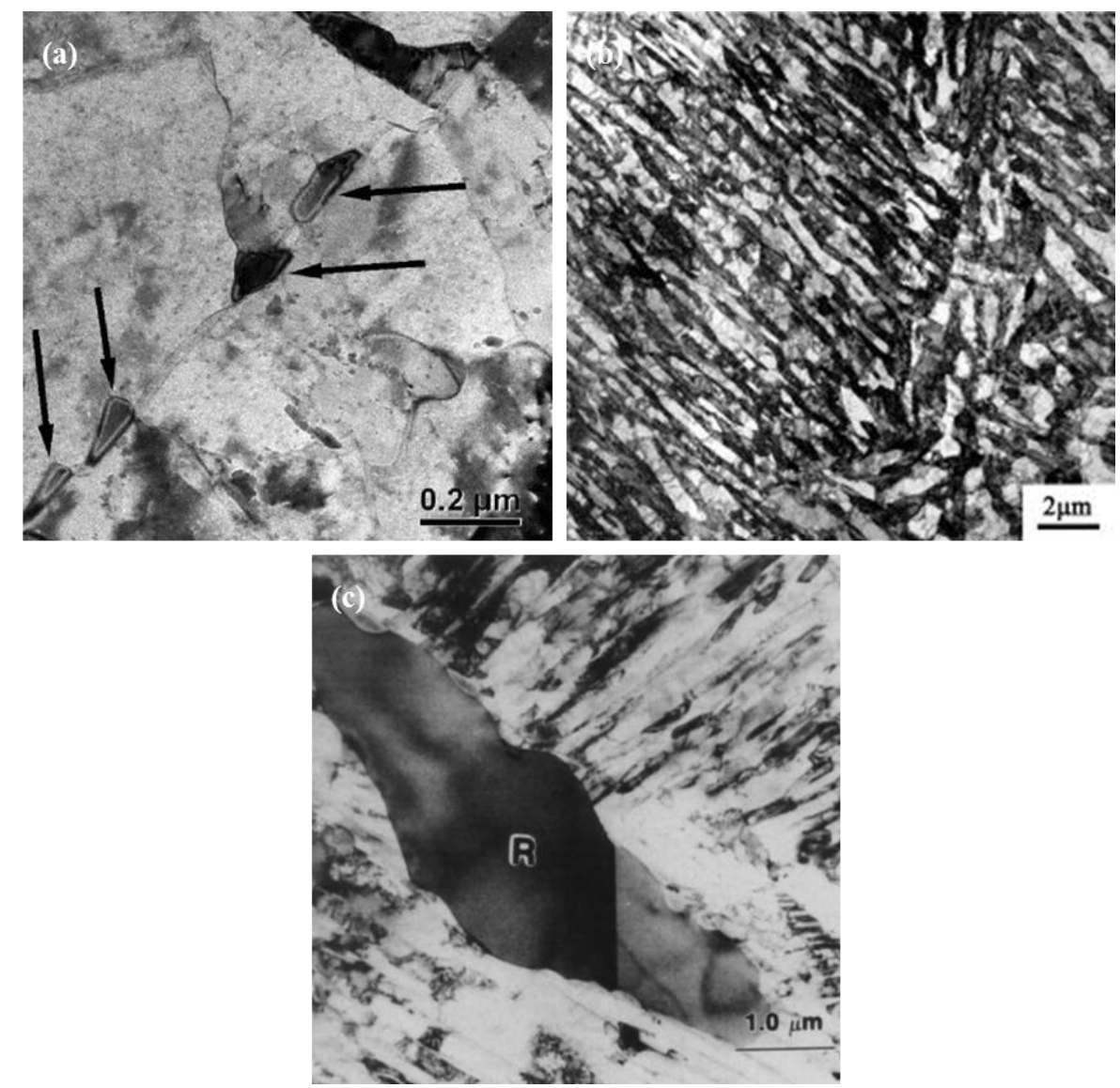

Figure 2-6 (a) Matrix austenite at prior austenite grain boundaries [147] (b) the lamellar structure of alternate lath-like austenite and residual martensite [27] and (c) a recrystallized austenite grain free of defects [146]. 


\subsubsection{Adjacent microstructure}

Many studies on TRIP and Q\&P steels reveal that the stability of retained austenite is also associated with the adjacent microstructure [142][164][165][173]. For example in TRIP steels, retained austenite in bainitic ferrite is found more stabilized than that in polygonal ferrite. Two possible mechanisms are given to explain this phenomenon. Grajcar [173] stated that the retained austenite surrounded by bainitic ferrite had a shorter diffusion path to carbon atoms and thus was more thermally stable. Another explanation is that the bainitic ferrite can stabilize retained austenite by stress partitioning. The stress partitioning mechanism points out that a harder phase adjacent to retained austenite can decrease the hydrostatic stress without affecting the equivalent stress, which in turn decreases the mechanical driving force for transformation as following [155]:

$$
\frac{\partial \Delta g^{\sigma}}{\partial \bar{\sigma}}=-0.715-0.3206 \frac{\sigma_{h}}{\bar{\sigma}}
$$

where $\sigma_{h}$ is the hydrostatic stress and $\bar{\sigma}$ is the equivalent stress. The harder bainite may restrain the phase transformation of retained austenite as the adjacent bainite would have to deform so as to coordinate the volume expansion resulting from the martensitic transformation. Therefore, compared to polygonal ferrite, the harder bainitic ferrite can better shield the retained austenite located nearby and suppress phase transformation. It is worth noting that the morphology of retained austenite in bainitic ferrite is different from that in polygonal ferrite which may also be a reason for the difference in stress partitioning.

A similar mechanism was proposed on the stability of retained austenite in a Q\&P steel [164]. According to their study, the martensitic transformation occurred at the onset of deformation in blocky retained austenite surrounded by proeutectoid ferrite; while film-like retained austenite in lath martensite, despite having a lower carbon content, is more stabilized against deformation. Apart from the martensite/bainite shielding effect, Jacques et al. [157] speculated that the small size effect of film-like retained austenite, may also contribute to the higher mechanical stability of retained austenite. The discussion on multi-phase steels is never simple as the mechanical stability of austenite is generally determined by more than one factor. 


\subsubsection{Crystallographic orientation relationships}

Different crystallographic orientation relationships have been found between fcc and bcc crystals. Three relationships, i.e., Kurdjumov-Sachs (K-S) relationship [52][146][174][175], Nishiyama-Wassermann (N-W) relationship [146][176][177] and Pitsch relationship [178] are the most commonly known relationships in steels. A summary of these orientation relationships is displayed in Table 2-1 [169]. The Bain orientation relationship is the simplest one among those relationships, but has rarely been observed in steels. It is shown here as a reference relationship when discussing the orientation relationship. The Pitsch orientation is usually regarded as 'inverse NW' orientation. To better explain these orientation relationships, the crystallographic structures are illustrated in Figure 2-7 [179]. The orientation relationships are interpreted by presenting the crystallographic planes and directions of the two phases. For example, in the case of Kurdjumov-Sachs (K-S) relationship, it is specified as one $\{111\}_{\gamma}$ plane being parallel to one $\{100\}_{\alpha}$ plane and a $<$ $100>_{\gamma}$ direction within the $\{111\}_{\gamma}$ plane being parallel to $\mathrm{a}<111>_{\alpha}$ direction within the $\{100\}_{\alpha}$ plane.

The numbers of variants for each orientation relationship are also given in Table 2-1. For example in K-S relationship, there are four different $\{111\}_{\gamma}$ planes, each $\{111\}_{\gamma}$ plane has three different $<110>_{\gamma}$ directions and each $<110>_{\gamma}$ has two different $<111>_{\alpha}$ directions which are parallel to it. Therefore, there are 24 variants for K-S orientation relationship in total. The variants of orientation relationships between fcc and bcc phases are important when analysing the twinned austenite in bcc ferrite.

$<\mathrm{uvw}>/ \omega_{\min }$, in the last column of Table $2-1$, are the parameters to describe the misorientation between two crystallographic orientations. As the most reported orientation relationships, the difference in angular between K-S and N-W relationships is small, so distinguishing the two relationships by misorientation in experiment is difficult. As shown in Figure 2-7, the $\{111\}_{\gamma}$ plane is parallel to the $\{011\}_{\alpha}$ plane in the two types of orientation relationships (K-S: Figure 2-7(b); N-W: Figure 2-7(c)) and the misorientation between the NW variants and any of the two neighbouring $\mathrm{KS}$ variants is only $5.26^{\circ}$. 
Table 2-1 Overview of the different orientation relationships between fcc and bcc crystals [169].

\begin{tabular}{cccc}
\hline & Orientation relationship & $\begin{array}{c}\text { Number of } \\
\text { variants }\end{array}$ & $<\mathrm{uvw} / \omega_{\min }$ \\
\hline Bain (B) [180] & $\{100\}_{\gamma} \|\{100\}_{\alpha}$ & 3 & $<100>/ 45$ \\
& $<100>_{\gamma} \|<110>_{\alpha}$ & & \\
Kurdjumov-Sachs & $\{111\}_{\gamma} \|\{110\}_{\alpha}$ & 24 & $<0.970 .180 .18>$ \\
(K-S) [174] & $<110>_{\gamma} \|<111>_{\alpha}$ & & $/ 42.85$ \\
Nishiyama-Wasserman & $\{111\}_{\gamma} \|\{110\}_{\alpha}$ & 12 & $<0.980 .080 .20>$ \\
(N-W) [176][177] & $<112>_{\gamma} \|<110>_{\alpha}$ & & $/ 45.98$ \\
Pitsch (P) [178] & $\{100\}_{\gamma} \|\{110\}_{\alpha}$ & 12 & $<0.080 .200 .98>$ \\
& $<110>_{\gamma} \|<111>_{\alpha}$ & & $/ 45.98$ \\
\hline
\end{tabular}

Given that the only difference between the two orientation relationships is the rotational angle, it is proposed that the K-S and N-W relationships may co-exist in steels. But studies are in debate on the relatively dominant position between K-S and N-W relationships in steels [143][168][181][182][183]. Katz et al. [184] reported both of the two orientation relationships existed between reverted austenite and martensite matrix in $18 \mathrm{Ni}$ maraging steels. Shiang and Wayman [146], who also noticed the two relationships, suggested that the dominant orientation relationship was correlated to the aging temperatures. Suk et al. [167] found that reverted austenite had a specific orientation relationship not only with the surrounding martensite but also with the adjacent precipitates. In addition, the twinned austenite, which has been observed in both maraging steel [185][186] and dual-phase stainless steel [187], was found obeying the K-S orientation relationship [167]. The N-W orientation relationship, however, has not been found yet between the twinned austenite and martensite. 
Although some researchers stated that the orientation effect of fcc phase on the strength is negligible [188][189][190], there are others who held a different view [166][191]. It is accepted that the local stress is heterogeneous at the microscopic level when the phase orientations and activated slip systems are taken into consideration. Wang et al., who studied the mechanical stability of reverted austenite in a $13 \% \mathrm{Cr}-4 \% \mathrm{Ni}$ martensitic steel, suggested that the stress at the $\alpha / \gamma$ interface with $\mathrm{N}$ or $\mathrm{K}-\mathrm{S}$ relationships was much lower than that without $\mathrm{N}$ or $\mathrm{K}-\mathrm{S}$ relationships [166] and they attributed the difference to the activation of the slip systems. The activation of slip system is normally affected by two factors. One is the external load, the other one is the interaction between phases. In the case of austenite/martensite, plastic strain occurs in the austenite earlier than martensite during deformation due to its lower critically resolved shear stress. Wang et al. [166] stated that when the slip system was activated in reverted austenite, it can penetrate the boundaries and activate the slip system in the adjacent martensite easily with the assist of $\mathrm{N}$ or K-S relationship. It means that if the special $\mathrm{N}$ or K-S relationship exists, a smaller slip strain in austenite is required to activate the slip systems of the surrounding martensite, and thus the local stress of austenite will decrease to a lower level. On the other hand, when the N-W or K-S relationship is absent, it needs higher stress and strain accumulation in austenite to activate the slip system of nearby martensite. However, the accumulated stress in austenite is highly likely to induce martensitic transformation and hence result in a lower mechanical stability of austenite. 
(a) $\{100\}_{\gamma}\left\|\{100\}_{\alpha},<100>_{\gamma}\right\|<110>_{\alpha}$

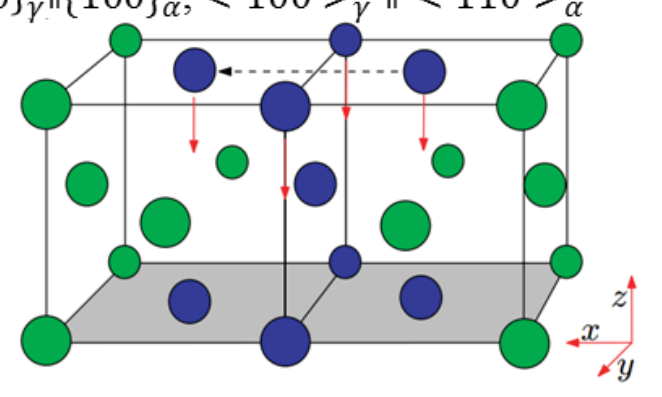

(b) $\{111\}_{\gamma}\left\|\{110\}_{\alpha},<110>_{\gamma}\right\|<111>_{\alpha}$

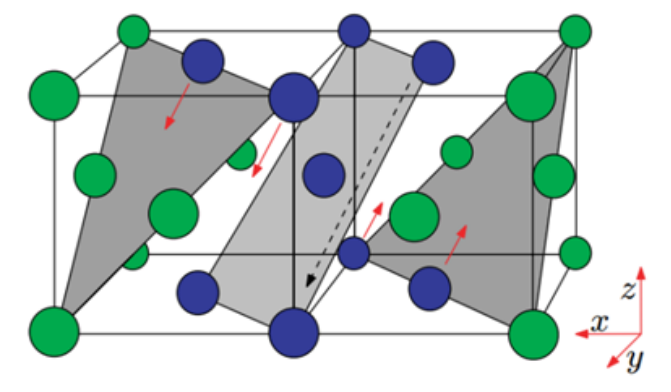

(c) $\{111\}_{\gamma}\left\|\{110\}_{\alpha},<112>_{\gamma}\right\|<110>_{\alpha}$

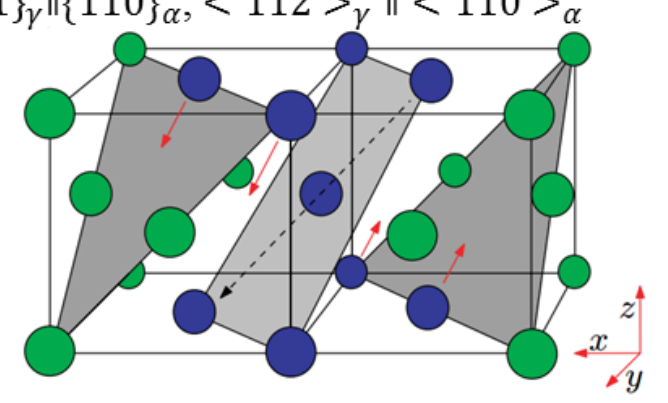

Figure 2-7 Different orientation relationships between fcc and bcc phases: (a) Bain, (b) K-S, and (c) N-W. Blue atoms belong to bcc unit cell and green atoms belong to fcc unit cell

[179].

\subsubsection{The formation mechanism of reverted austenite}

It has been acknowledged that the formation of granular reverted austenite is associated with a diffusion-controlled process. The granular reverted austenite is deemed to result from the dissolving of cementite or precipitates which leads to the enrichment of austenite stabilizing elements in localized areas [27][147][192]. On the other hand, the formation mechanism of lath-like reverted austenite is 
controversial [146]. Plichata and Aaronson supposed that the growth of lath-like austenite was also controlled by diffusion [193]. Sinha et al. [194] further pointed out that, instead of precipitate dissolution, the diffusion of $\mathrm{Ni}$ atoms in matrix to dislocations or other defects generated the local enrichment of austenite stabilizing elements. Schnitizer et al. [147], Nakagawa et al.[20] and Sinha et al. [194] reported the value of activation energy for reverted austenite formation was about 234-250 $\mathrm{kJ} / \mathrm{mol}$ which is in the range of the activation energy of Ni diffusion in pure Fe (246 $\mathrm{kJ} / \mathrm{mol}$ [195]). Conversely, some researchers suggested the formation of lath-like reverted austenite to be a shear-dominated process but assisted by a diffusion mechanism [52][146]. They believed that due to the segregation of solute elements to preferential locations, the reverse transformation start temperature $\left(A_{s}\right)$ at these sites reduces to below the aging temperature. Phase transformation involving shear deformations, which is also referred to as displacive phase transformation, is expected to occur in the similar way as bainitic transformation [172]. The lattice correspondence for this phase transformation normally obeys $\mathrm{K}-\mathrm{S}$ or $\mathrm{N}-\mathrm{W}$ relationships. Besides, the twinned lath-like austenite in some maraging steels is another evidence for the occurrence of a shear motion in austenite reversion [146][172]. Lee et al., who studied the austenite reversion in both $\mathrm{Fe}-3 \mathrm{Si}-13 \mathrm{Cr}-7 \mathrm{Ni}$ (wt.\%) martensitic stainless steel and medium Mn steels, suggested that the formation mechanism of reverted austenite was heating-rate related. Below the critical heating rate $(10 \mathrm{~K} / \mathrm{s}$ for $\mathrm{Fe}-3 \mathrm{Si}-13 \mathrm{Cr}-7 \mathrm{Ni}$ martensitic stainless steel and 15 $\mathrm{K} / \mathrm{s}$ for medium Mn steels), the austenite reversion process was diffusion-controlled and the reverted austenite presented a globular shape with a diameter of 200-250 nm. Besides, a lower dislocation density and a higher Mn concentration were observed in this type of reverted austenite. When the samples were heated faster than the critical heating rates, the phase transformation occurred by a diffusionless shear mechanism. Reverted austenite presented a lath-like shape with 200-300 nm in width and 400$700 \mathrm{~nm}$ in length. They also mentioned that despite the diffusionless reverted austenite had a high density of dislocation, it yielded lower mechanical stability owing to the lower Mn concentration and larger grain size. In addition, it is worth noting that, $\alpha^{\prime} \rightarrow \gamma$ transformation as well as bainitic transformation [196] is not 
universally accepted to be shear-controlled. Further studies on the mechanism of $\alpha^{\prime} \rightarrow \gamma$ transformation are needed to support this viewpoint.

It is suggested that some transformation-induced deformation may be induced during the formation of reverted austenite, and the stored strain energy may act as a driving force to cause recrystallization [146]. For steels in which retained austenite also exists, austenite reversion is governed by the competition between the nucleation of new reverted austenite and the growth of existing retained austenite. There are two mechanisms relating to the $\alpha^{\prime} \rightarrow \gamma$ transformation, i.e. abnormal $\alpha^{\prime} \rightarrow \gamma$ transformation and normal $\alpha^{\prime} \rightarrow \gamma$ transformation [192]. The abnormal $\alpha^{\prime} \rightarrow \gamma$ transformation proposes that the retained austenite grains within one prior austenite grain normally have the same orientation, therefore their growth may result in the coalescence and further, a reconstruction of the original prior austenite grain boundaries (Figure 2-8(a)). This process is called as the austenite grain memory effect (also known as austenite recrystallization). While the normal $\alpha^{\prime} \rightarrow \gamma$ transformation proposes that newly formed reverted austenite cannot coalesce as these austenite grains have different orientations.

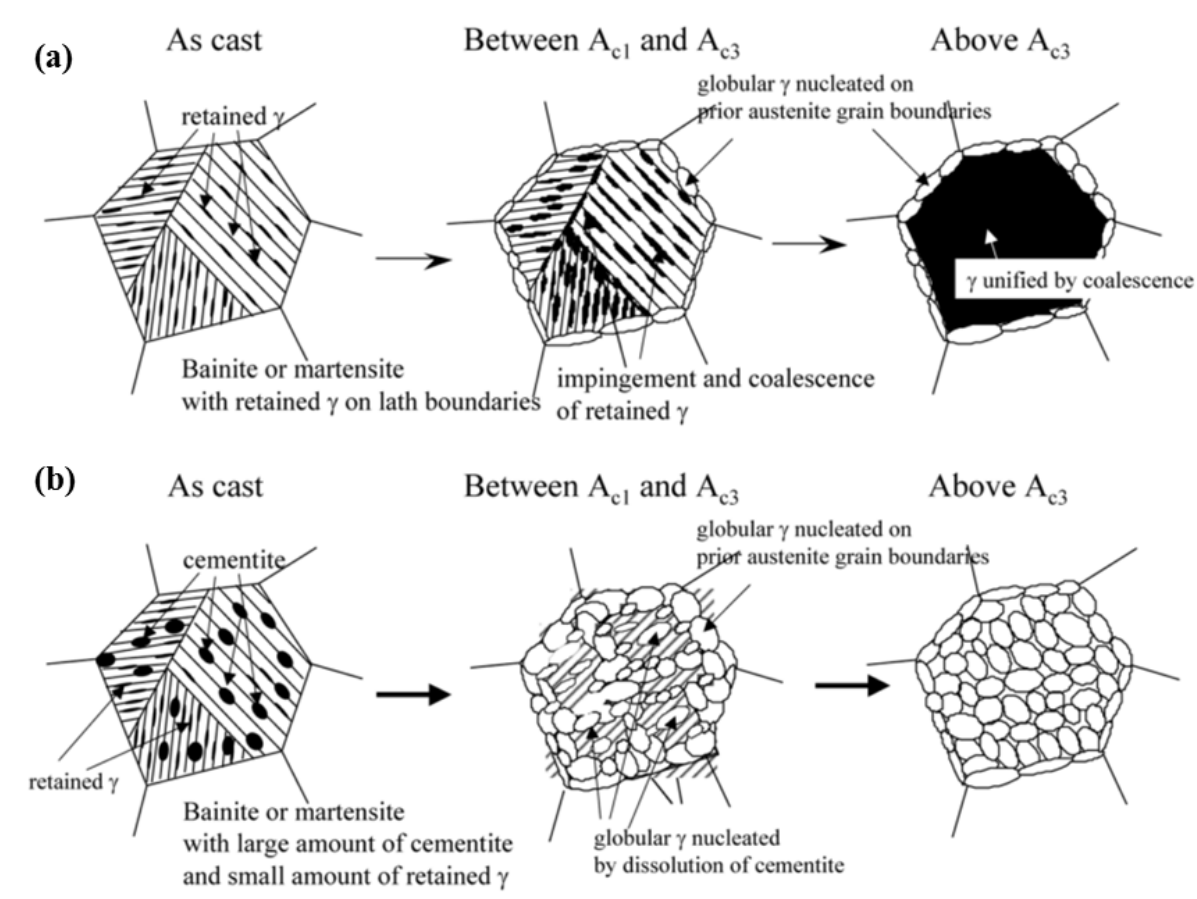

Figure 2-8 Schematic illustration of (a) abnormal $\alpha^{\prime} \rightarrow \gamma$ transformation and (b) normal $\alpha^{\prime} \rightarrow \gamma$ transformation [192]. 


\subsection{Grain refinement in maraging steels}

It is commonly known that there is an inverse relationship between strength and toughness. One of the methods to improve both simultaneously is the grain refinement. The famous Hall-Petch equation expresses the relationship between yield stress and grain size [197]:

$$
\sigma_{y}=\sigma_{0}+k_{y} d^{-1 / 2}
$$

where $\sigma_{y}$ is the yield stress, $\sigma_{0}$ is the intrinsic friction stress of an undeformed single crystal oriented for multiple slip, $k_{y}$ is the Petch constant representing the resistance against the slip propagation across grain boundary and is generally specific to materials, $d$ is the grain size [198].

The fracture strength, which is derived from dislocation theory [199][200] and classical Griffith theory [201], is an indication of the resistance to the propagation of a crack around a dislocation pile-up grain boundary:

$$
\sigma_{f}=4 G \gamma_{m} k_{y}^{-1} d^{-1 / 2}
$$

where $\sigma_{f}$ is the fracture strength, $G$ is the shear modulus, $\gamma_{m}$ is the surface energy. According to Equation (2.12), it is apparent that both the yield stress and fracture strength increase when the grain size is refined.

In addition, since $\sigma_{0}$ is strongly dependent on the temperature, $\sigma_{y}$ increases

significantly as the temperature reduces; whereas $\sigma_{f}$ is much less affected by the temperature. Consequently, the critical DBTT, $T_{c}$, is introduced when $\sigma_{y}=\sigma_{f}$ as:

$$
T_{c}=C^{-1} \ln \left[B k_{y} d^{1 / 2} /\left(\beta G \gamma_{m}-k_{y}^{2}\right)\right]
$$

where $C, B$ and $\beta$ are constants [202]. According to Equation (2.13), $T_{c}$ can also be decreased by refining grain size.

\subsubsection{Conventional methods to refine grains}

Thermomechanical processing, as one of the most well-known methods for grain refinement, has been intensively studied in various alloys. It has been demonstrated 
that the extraordinary improvement in both strength and toughness can be obtained in steels by refining grain size to $<1 \mu \mathrm{m}$ via thermomechanical processing [203]. However, in the steel industry it is difficult to perform a uniform plastic deformation through the thickness of plates to produce uniform ultrafine grain size [204]. Therefore, in the case of thick plate steels (or bars and weldments, etc.) with high strength, alternative methods such as cyclic heat treatment are introduced to refine grain size.

\subsubsection{Effective grain size in lath martensitic steels}

In ferritic steels, as the cleavage normally occurs along $\{100\}_{\alpha}$ planes [204], the effective grain size, in terms of transgranular cleavage fracture, is determined as the coherent length on $\{100\}_{\alpha}$ planes, or the average free path of crack propagation along $\{100\}_{\alpha}$ planes [203].

In martensitic steels, the definition of effective grain size is more complicated. The interfaces in quenched martensite can be classified into several types: prior austenite, martensite packet, block and lath boundaries. They generally possess different energies, structures, misorientations, mechanics and segregation properties [50]. The effective grain size is correlated to the prior thermal processes. A typical microstructure of martensitic steel is illustrated in Figure 2-9. The prior austenite grains are divided into packets of fine, parallel distributed martensite laths (Figure 2-9(b)). The thickness of laths is generally at nano-scale. Electron diffraction studies revealed that the laths within a packet are in close crystallographic alignment and the lath boundaries are low-angle boundaries [204]. It means that the martensite packet is actually a single crystal. Therefore, the size of the packet is defined as the effective grain size of lath martensite. 

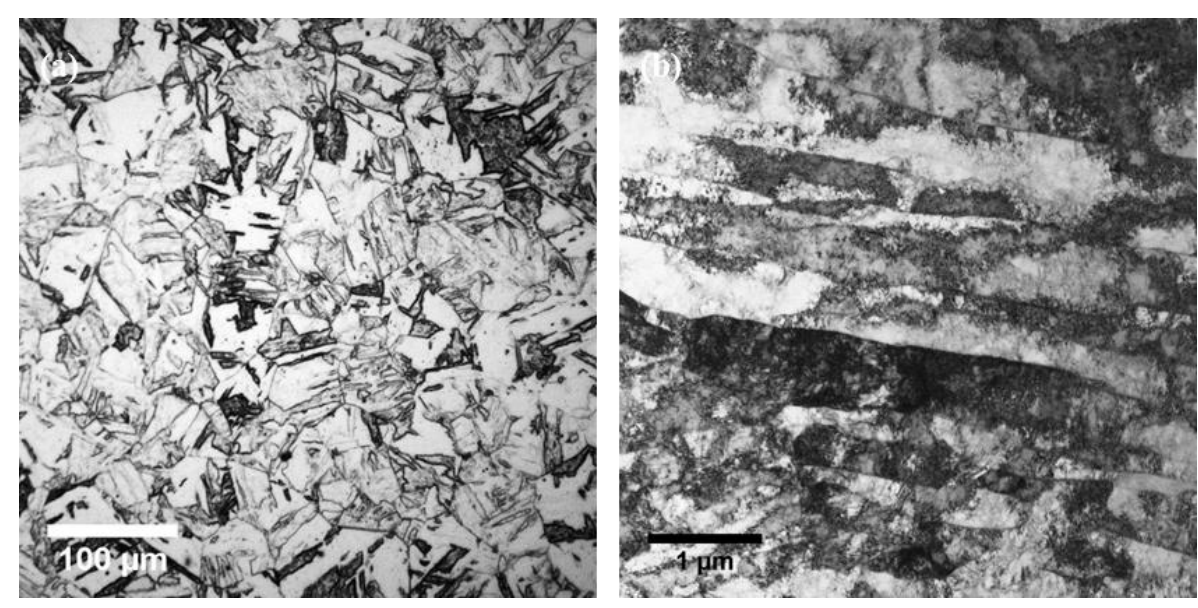

Figure 2-9 (a) The typical microstructure of a martensitic steel constituted by packets of martensite laths, (b) the dislocated laths which is at nano-scale in thickness. Both are the micrographs of a $10 \% \mathrm{Mn}$ maraging steel after water quenching from solution heat treatment.

\subsubsection{Methods of grain refinement in lath martensitic steel}

If taking account of the effective grain size of lath martensite, there are mainly three approaches to refine the grain size of martensitic steel. The most common method is by refining the prior austenite grain size. The practical heat treatment to realize it is by quenching from austenization before the austenite grains coarsen, but it has been demonstrated that this method can hardly refine the prior austenite grain size further below $10 \mu \mathrm{m}$ [203].

A more direct method is to generate high-angle misorientation between the adjacent laths and thereby breaking up the crystallographic alignment of laths within the packets [205]. Although this method seems to be the most direct way to completely refine the effective grain size, it is difficult to control in production. This is owing to the difficulty to design the martensitic transformation and the crystallographic relationships of martensite laths by thermal treatment.

Based on the second approach, the third approach for grain refinement is to form a new phase at boundaries, such as austenite, so as to separate the martensite laths. This type of austenite islands is required to be very thin and remain stable during heat treatment. This is generally achieved by intercritical tempering (in most cases, a 
temperature just above $A_{c 1}$ is desirable). This mechanism of improving toughness via austenite reversion is a matter of debate. One possible explanation is that the formation of austenite, which generally has $\mathrm{K}-\mathrm{S}$ or N-W orientation relationships with adjacent martensite, introduces high-angle misorientation between phases. Even when the metastable austenite transforms into martensite during deformation, the fresh martensite with different variant can still disrupt the crystallographic alignment of laths. This method has been widely used to in medium-Ni and medium-Mn steels [27][24][156][206] by Q\&P treatment or in maraging steels by aging process [18][19][145][146]. 


\section{Chapter 3 Materials and Experimental Methods}

\subsection{Alloys and processing details}

\subsubsection{Alloys and Composition Analysis}

The present study is mainly focused on investigating the microstructure and mechanical properties of Mn-based maraging TRIP steels and hereby developing new alloy compositions to improve the properties. Three as-cast steels, namely, two (10 wt.\% Mn, 12 wt.\% Mn) based on the compositions mentioned in Ref. [18] with minor addition of $\mathrm{Al}(1 \mathrm{wt} . \%)$ and one with lower $\mathrm{Mn}$ content (7 wt.\% Mn) were provided by Tata Steel. The chemical compositions of alloys are listed in Table 3-1. The low carbon content $(<0.04$ wt. $\%)$ is to achieve a soft but heavily dislocated martensite after water quenching. Minor elements, such as $\mathrm{Ni}, \mathrm{Mo}$, $\mathrm{Ti}$ and $\mathrm{Al}$ are added to form precipitates. Compared with the conventional $18 \mathrm{Ni}$ maraging steels, the main difference exists in the content of austenite stabilizer Mn. 7-12 wt.\% Mn are added to ensure that an appropriate amount of austenite is obtained with appropriate stability and yet being substantially lower than the Mn contents in the twinning-induced plasticity (TWIP) steels.

Table 3-1 Chemical compositions (wt.\%) of the investigated Mn-based steels

\begin{tabular}{ccccccccccc}
\hline Materials & $\mathrm{C}$ & $\mathrm{Mn}$ & $\mathrm{Ni}$ & $\mathrm{Mo}$ & $\mathrm{Al}$ & $\mathrm{Ti}$ & $\mathrm{Si}$ & $\mathrm{Cr}$ & $\mathrm{Co}$ & $\mathrm{Fe}$ \\
\hline $7 \% \mathrm{Mn}$ & 0.033 & 7.07 & 2.03 & 1.03 & 1.04 & 1.05 & 0.07 & $<0.005$ & $<0.001$ & bal. \\
$10 \% \mathrm{Mn}$ & 0.015 & 9.97 & 2.03 & 1.19 & 1.00 & 0.84 & 0.07 & 0.02 & 0.008 & bal. \\
$12 \% \mathrm{Mn}$ & 0.022 & 11.77 & 1.97 & 1.17 & 1.00 & 0.82 & 0.08 & 0.03 & 0.009 & bal.
\end{tabular}

\subsubsection{Hot rolling and heat treatment procedure}

The equilibrium $\gamma / \alpha$ transformation temperatures $\left(\mathrm{Ae}_{3}\right)$ of $7 \%, 10 \%$ and $12 \% \mathrm{Mn}$ alloys were calculated as $722{ }^{\circ} \mathrm{C}, 644{ }^{\circ} \mathrm{C}$ and $614{ }^{\circ} \mathrm{C}$, respectively. In order to 
minimize the detrimental effect of coarse carbide, the three ingots were first homogenized under argon gas atmosphere at $1150{ }^{\circ} \mathrm{C}$ for $1 \mathrm{~h}$ and hot rolled between $1140{ }^{\circ} \mathrm{C}$ and $850{ }^{\circ} \mathrm{C}$. The thickness of ingots was reduced from $20 \mathrm{~mm}$ to $3 \mathrm{~mm}$ with six passes and the corresponding reduction ratio was $85 \%$. Inter-stage re-heating to $1150{ }^{\circ} \mathrm{C}$ was performed after every two passes. The detailed parameters for hot rolling process are presented in Table 3-2. The subsequent solution heat treatment (SHT) was carried out with argon protection at $1050{ }^{\circ} \mathrm{C}$ followed by water quenching. After SHT, the samples were then aged at $420{ }^{\circ} \mathrm{C}, 460^{\circ} \mathrm{C}$ and $500{ }^{\circ} \mathrm{C}$ for a range of times from 10 minutes to 168 hours (Figure 3-1).

Table 3-2 Parameters for hot rolling process

\begin{tabular}{ccccccc}
\hline Temperature $\left({ }^{\circ} \mathrm{C}\right)$ & 1140 & 1040 & 1090 & 950 & 1030 & 860 \\
\hline $\begin{array}{c}\text { Thickness reduction } \\
(\mathrm{mm})\end{array}$ & $20-14$ & $14-10$ & $10-7$ & $7-5$ & $5-4$ & $4-3$ \\
$\begin{array}{c}\text { Reduction ratio } \\
\text { (30\% }\end{array}$ & $28.6 \%$ & $30 \%$ & $28.6 \%$ & $20 \%$ & $25 \%$ \\
\hline
\end{tabular}

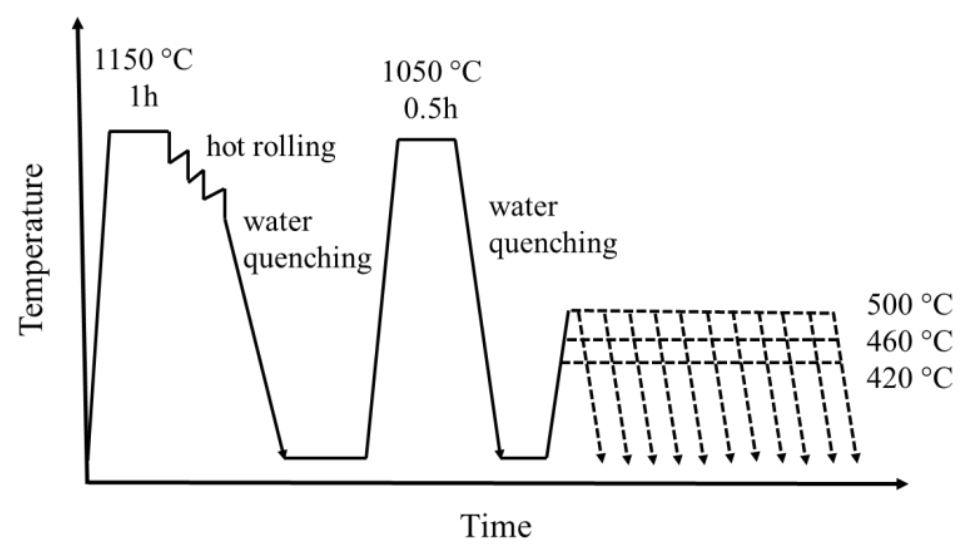

Figure 3-1 Schematic diagram of processing conditions 


\subsection{Sample preparation}

\subsubsection{Mircopreparation}

All hot-rolling and heat-treatment pieces for characterization were cut from bulk samples after each processing procedure. To avoid deformation-induced phase transformation, all the aged materials were cut by Isomet $^{\mathrm{TM}} 5000$ with feeding speeds of 2-4 mm/s. Small pieces of samples were then hot mounted with Bakelite for optical and scanning electron microscopy (SEM) observation. Samples without Bakelite were prepared for X-ray diffraction (XRD) and transmission electron microscopy (TEM) analyses. The subsequent mechanical grinding and polishing process needed to be carefully controlled as any inappropriate operation may induce undesired phase transformation. The grinding operation was carried out successively on 240, 400, 800 and 1200 grade silicon carbide papers with running water. The manual polishing operation was performed on $6 \mu \mathrm{m}$ and $1 \mu \mathrm{m}$ polishing cloth with corresponding diamond suspensions. Some techniques such as nanoindentation and electron backscatter diffraction (EBSD) demand an excellent sample surface finish, in these cases, $0.04 \mu \mathrm{m}$ colloidal silica (silco) suspension was used for the final polishing. Silco suspension has a slight etching effect, thereby being able to remove the residual strain layer from the manual grinding and polishing procedure. The load, rotation speed and duration for each step of grinding and polishing varied depending on the surface condition. Generally a small load $(10 \mathrm{~N})$ for approximately 5 minutes was desired for final polishing. All the samples were rinsed and dried with isopropanol after each step and final cleaning was done using ultrasonic agitation.

\subsubsection{Etching}

The $2 \%$ nital is the most widely used etchant for general steels. In this study, $2 \%$ nital worked effectively on hot-rolled and solution-treated samples, but failed to reveal the reverted austenite in aged samples. It was eventually found that picric acid can clearly distinguish reverted austenite from martensite matrix. The picric acid etchant was a mixed solution of $75 \mathrm{~mL}$ saturated picric acid and $25 \mathrm{~mL}$ wetting agent. The etching process was performed at $60-70{ }^{\circ} \mathrm{C}$ for a few seconds. The etching 
temperature and duration was dependent on the amount of reverted austenite. Normally, a lighter etch than the conventional picric acid etch for the observation of prior austenite grain boundaries observation was more appropriate in this study.

\subsubsection{TEM thin foil preparation}

TEM thin foil samples were prepared by two methods, in which electropolishing was the mainly used one in this study. The bulk samples were manually ground to foils below $150 \mu \mathrm{m}$ thick. Disks with a diameter of $3 \mathrm{~mm}$ were then punched and further thinned to 50-80 $\mu \mathrm{m}$. Electropolishing was conducted with 5\% perchloric acid, $35 \%$ butoxyethanol and $60 \%$ methanol solution (maintained at approximately $-40{ }^{\circ} \mathrm{C}$ by a liquid nitrogen cooling system) running through a twin-jet electropolisher operated at 25-35 V (being adjusted to ensure the current of $\sim 40 \mathrm{~mA}$ ). The whole process lasted approximately 2-3 minutes depending on the operation parameters (such as temperature, jet speed and sensitivity, etc.) and stopped once the foil was perforated. Then the foil was cleaned immediately and stored by methanol.

High quality TEM thin foil can be obtained with carefully controlled parameters for most of steel samples. However, in this study, reverted austenite in aged samples was found disappeared after electropolishing. In this case, a combination of electropolishing with ion milling techniques was employed. Thin foils were thinned by electropolishing and stopped manually before perforation. Then ion milling was performed on Gatan model 600 or Fischione model 1010 for a few hours. Usually a voltage of $3 \mathrm{kV}$ and milling angle of $6^{\circ}$ were used for final thinning. Ion milling can be utilized to remove contamination and oxide layer before TEM observation as well.

\subsubsection{Carbon extraction replicas}

Precipitates, which are normally at the nano-scale size range, can be easily recognized under TEM by using carbon extraction replica, and thus be characterized by selected area electron diffraction (SAED) and energy-dispersive X-ray spectroscopy (EDS) in the absence of bulk matrix. The matrix is generally considered to absorb or overlap the faint signals of precipitates due to their small amount. In order to conduct accurate investigation on nano-size precipitates without 
interference from the surrounding matrix (especially in this study the electron beam was distracted by the magnetic field introduced by martensite), carbon extraction replicas were prepared and examined by TEM. Firstly, the polished sample was cleaned carefully by ultrasonic agitation to exclude the particles retained from diamond polishing suspension. Precipitates embedded in the matrix were revealed by etching lightly with picric acid (at $60-70{ }^{\circ} \mathrm{C}$ ) for approximately 10 seconds. The sample was then coated with amorphous carbon film, usually a thin carbon film is desired for TEM analysis. The carbon film which evaporated small particles from the matrix was scratched into $2 \mathrm{~mm}$ squares. Then the sample was immersed into $10 \%$ nital to dissolve the matrix until the carbon film started to bubble and left the surface with the particles (Figure 3-2) [207]. The 400 square copper meshes were then used to catch those carbon film squares.

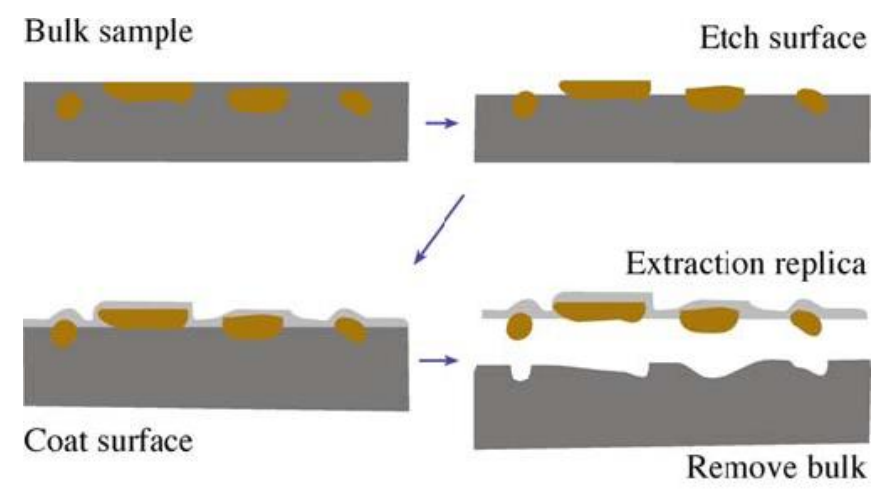

Figure 3-2 Schematic steps of making the carbon extraction replication [207].

\subsection{Characterization techniques}

\subsubsection{Qualitative and quantitative analyses by $\mathrm{X}$-ray diffraction (XRD)}

Samples of hot-rolled, solution-treated and aged steels were examined by XRD for phase identification and evaluating the volume fraction of austenite. Samples were cut into pieces with a size of $20 \mathrm{~mm} \times 20 \mathrm{~mm} \times 3 \mathrm{~mm}$. The subsequent metallographic preparation has been described in Section 3.2. 
X-ray diffraction work was performed on Siemens D500 Diffractometer using Co $\mathrm{K}_{\alpha}$ radiation $(\lambda=1.78897 \AA)$ and Siemens $\mathrm{D} 5000 \mathrm{using} \mathrm{Cu} \mathrm{K} \alpha$ radiation $(\lambda=1.54178$ $\AA$ ). Both two diffractometers were operated at the condition of $40 \mathrm{kV}$ and $40 \mathrm{~mA}$. Samples were step scanned $\left(0.02^{\circ}\right.$ per step) by a beam size of $0.2 \mathrm{~mm}$ covering a range of $2 \theta$ angles. For quantitative phase analysis, more than two sites of each sample were scanned. XRD data were collected by WinXPOW (D500) and DIFFRAC plus XRD commander (D5000), respectively. The analyses were undertaken using ICDD PDF-4+.

X-ray diffraction (XRD) measurement was applied to identify the austenite (facecentred cubic, fcc) in martensite matrix (body-centred tetragonal, bct). It is worth noting that, to simplify the discussion, $\alpha^{\prime}$-martensite was treated as ferrite (bodycentred cubic, bcc) thermodynamically and kinetically in this study. But elevated elemental diffusivity should be taken into consideration when studying the kinetics of phase formation. The XRD patterns were compared to standard diffraction patterns by software to identify phases. Analogous to the illustration in Section 3.3.4.2, each phase has characteristic X-ray peaks which allow them to be identified. The accurate $d$-spacings were calculated using Bragg's law, $\lambda=2 \mathrm{~d} \sin \theta$ ( $\lambda$ is the wavelength of target radiation and $\theta$ is half of the angle where the peak presents).

XRD technique is considered as one of the most accurate and convenient methods to evaluate the volume fraction of austenite in steels. In this study, the volume fraction of austenite was calculated via comparison method described by Yang et al. [208] and the ASTM standard E975-03 [209]. Specific XRD patterns for each crystalline phase were produced during irradiation. For example, $\alpha^{\prime}$-martensite (211) crystalline plane peaks at $82^{\circ}$ while austenite $(220)$ crystalline plane peaks at $74^{\circ}$ when a Co target is used. The volume fraction of austenite was measured by comparing the integrated intensity of austenite and $\alpha^{\prime}$-martensite. For a randomly oriented sample, the integrated intensities of the austenite $(\gamma)$ and ferrite $(\alpha)$ diffraction peaks are determined by:

$$
I_{\alpha}^{h k l}=\frac{K R_{\alpha}^{h k l} V_{\alpha}}{2 \mu}
$$




$$
I_{\gamma}^{h k l}=\frac{K R_{\gamma}^{h k l} V_{\gamma}}{2 \mu}
$$

where $K$ is the constant related to the instrumentation geometry and radiation. $\mathrm{R}$ is proportional to the theoretical integrated intensity which depends on the interplanar spacing $(h k l)$, Bragg angle $\theta$, crystal structure and composition of the phase. $V$ is the volume fraction of each phase and $\mu$ is the linear absorption coefficient of steels.

Therefore, any pair of ferrite and austenite $h k l$ peaks can be written as

$$
\frac{I_{\alpha}^{h k l}}{I_{\gamma}^{h k l}}=\frac{R_{\alpha}^{h k l} V_{\alpha}}{R_{\gamma}^{h k l} V_{\gamma}}
$$

For steels which contain carbides (or intermetallic precipitates in this study),

$$
V_{\alpha}+V_{\gamma}+V_{c}=1
$$

The volume fraction of austenite can be described as

$$
V_{\gamma}=\frac{1-V_{c}}{1+\frac{R_{\gamma}^{h k l}}{R_{\alpha}^{h k l}} \frac{I_{\alpha}^{h k l}}{I_{\gamma}^{h k l}}}
$$

It is implied by Equation (3.5) that the volume fraction of austenite is not much influenced by the presence of carbide. For example, when the volume fraction of carbide is 10 vol.\%, the volume fraction of austenite is calculated as 9 vol.\% by Equation (3.5). While if the carbide is ignored $\left(V_{c}=0\right)$, the volume fraction of austenite is calculated as $10 \%$. But the volume fraction of carbide has to be taken into account when it is over $15 \mathrm{vol} . \%$. In this case, the volume fraction of carbide is normally measured by chemical extraction and metallographic methods or currently TEM and APT techniques are utilized to obtain a more accurate result.

In this study, the integrated intensities of ferrite peaks (200) and (211) and austenite peaks (200), (220) and (311) were measured by ICDD PDF-4+. Calculated theoretical intensity ratios $\frac{R_{\gamma}^{h k l}}{R_{\alpha}^{h k l}}$ using Co $\mathrm{K}_{\alpha}$ radiation are illustrated in Table 3-3 [208].

If assuming $V_{c}=0$ in Equation (3.5), the volume fraction of austenite can thus be averaged from 6 pairs of ferrite and austenite peaks. 
Table 3-3 Calculated theoretical intensity ratios $\frac{R_{\gamma}^{h k l}}{R_{\alpha}^{h k l}}$ using Co $\mathrm{K}_{\alpha}$ radiation [208]

\begin{tabular}{cccc}
\hline & $(200)_{\gamma}$ & $(220)_{\gamma}$ & $(311)_{\gamma}$ \\
\hline$(200)_{\alpha}$ & 2.46 & 1.32 & 1.78 \\
$(211)_{\alpha}$ & 1.21 & 0.65 & 0.87 \\
\hline
\end{tabular}

\subsubsection{Grain size analysis by optical microscopy}

Hot-rolled and solution-treated samples for optical observation were prepared as described in Section 3.2.1 and 3.2.2. Optical observation was then undertaken on a Polyvar Optical Microscope to image the prior austenite grains and martensite packets.

Grain size in this study was measured using the linear intercept method. The principle of this method is to count numbers of grain boundaries per unit length. First, parallel lines are drawn on the image of the microstructure as illustrated in Figure 3-3. The number of times each line cross grain boundaries is counted. Thus, the average grain size $\bar{L}$ is given by:

$$
\bar{L}=\frac{L_{T o t a l}}{N_{L}}
$$

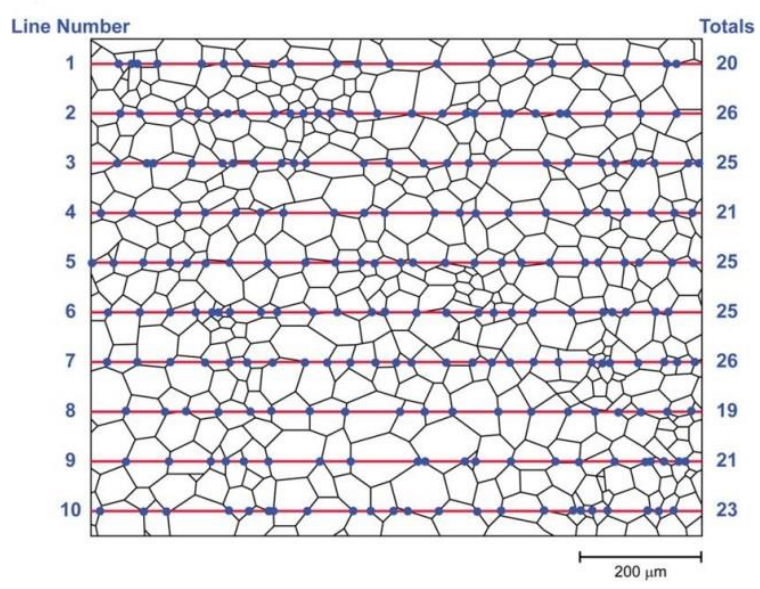

Figure 3-3 Schematic diagram of linear intercepts for the measurement of grain size. 
For the grains which are equiaxed, parallel lines can be drawn along random orientation as long as there is sufficient space larger than the grain size between lines. When the grains are not equiaxed, such as deformed materials, lines should be drawn in the rolling direction and the short transverse direction separately. The average grain size should be measured in two separate directions as well.

\subsubsection{Scanning electron microscopy}

The aged samples for SEM observation were manual prepared with the same standard as samples for optical observation. The picric acid etch was used to reveal reverted austenite and precipitates. The fracture behaviour of the three alloys in different conditions was also investigated by SEM. The fresh fracture surfaces of samples after tensile testing were examined to provide information about the fracture modes and the cause of fracture. In order to further study the damage mechanisms in microscopic level, the microstructure of the fractured samples was also observed by SEM. In addition, the volume fraction of reverted austenite after tensile tests was analysed by X-ray diffraction.

A FEI InspectF FEG-SEM equipped with EDS was utilized for both imaging and EDS analysis. The working conditions were dependent on the characterization purposes. An operating voltage of $15 \mathrm{kV}$ was usually used for microstructural observation and EDS analyses, whereas $10 \mathrm{kV}$ was usually set for fractography.

\subsubsection{Transmission electron microscopy}

TEM technique was mainly used to analyse the nanoscale features like precipitate and reverted austenite in this study. The morphology, size distribution and composition of precipitates and reverted austenite were examined by conventional transmission electron microscopes. Due to the overlap of diffraction spots between fine precipitates and bcc matrix, high resolution electron microscopy (HREM) was used to explore precipitates. TEM observation was conducted on four different microscopes which are Philips EM 420 operated at $120 \mathrm{kV}$ (for SAED analysis), FEI Tecnai operated at $200 \mathrm{kV}$ (for microstructural observation and EDS analysis), JEOL $2010 \mathrm{~F}$ at $200 \mathrm{kV}$ (HREM and TEM-EDS) and aberration-corrected FEG JEOL 
Z3100R05 at $300 \mathrm{kV}$ (HREM and scanning transmission electron microscopy (STEM)).

\subsubsection{Identification of the crystal structure by SAED}

The crystal structure of precipitates is of vital importance for the study of precipitation-strengthened materials as the structure of precipitates, to a large extent, determines their properties and thus their roles in precipitation-strengthened materials. SAED is one of the most frequently used experimental tools to identify the crystal structure of ultrafine phases which cannot be detected by XRD or SEM. Electron diffraction pattern is unique to the crystal structure and the patterns can provide information about the size and shape of the unit cells and atomic positions in the unit cell as well.

It is known that Bragg's conditions are satisfied when the Ewald sphere cuts a reciprocal lattice point specified by the indices of the reflecting plane, see Figure 3-4.

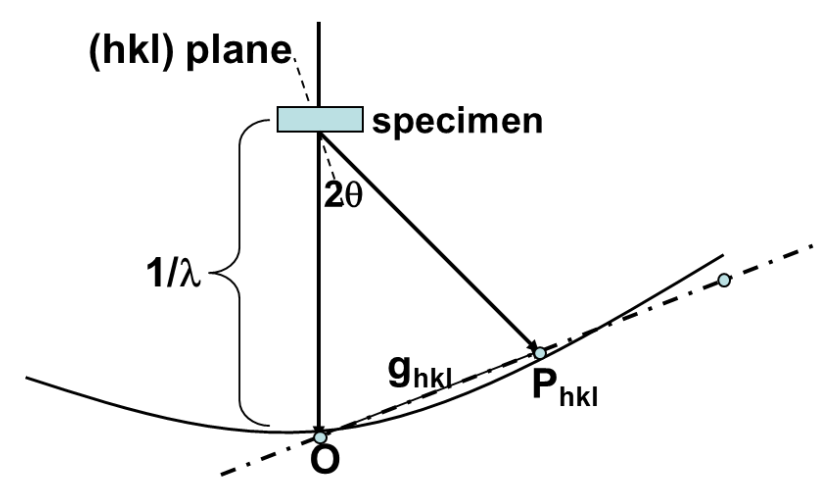

Figure 3-4 The construction of Ewald sphere after [207].

The Bragg's law is given as

$$
\sin \theta=\frac{g / 2}{1 / \lambda}=\frac{\lambda}{2 d_{h k l}}
$$

where $\mathrm{O}$ is the origin of the reciprocal lattice;

$\mathrm{P}_{\mathrm{hkl}}$ is the points of reciprocal lattice; 
$\lambda$ is the wavelength of the electrons, which is determined by the accelerating voltage.

$d_{h k l}$ is the crystal lattice spacing between atomic planes;

$\theta$ is the angle between the incident beam and the reflecting planes.

As Figure 3-5 reveals, the distance ( $r$ ) between the transmitted beam and diffracted beam as measured on the screen is determined by the camera length $L$,

$$
\tan 2 \theta=\frac{r}{L}
$$

when $\frac{1}{\lambda} \gg g$, it can be expressed as,

$$
\tan 2 \theta \approx \sin 2 \theta \approx 2 \theta
$$

combined with the Bragg equation (Equation (3.7)), $d_{h k l}$ can be obtained

$$
d_{h k l}=\frac{L \lambda}{r}
$$

$L \lambda$ is known as camera constant.

After measuring the nearest $r_{1}, r_{2}, r_{3}$, crystal lattice spacing $d_{1}, d_{2}, d_{3}$ can be calculated using Equation (3.10).

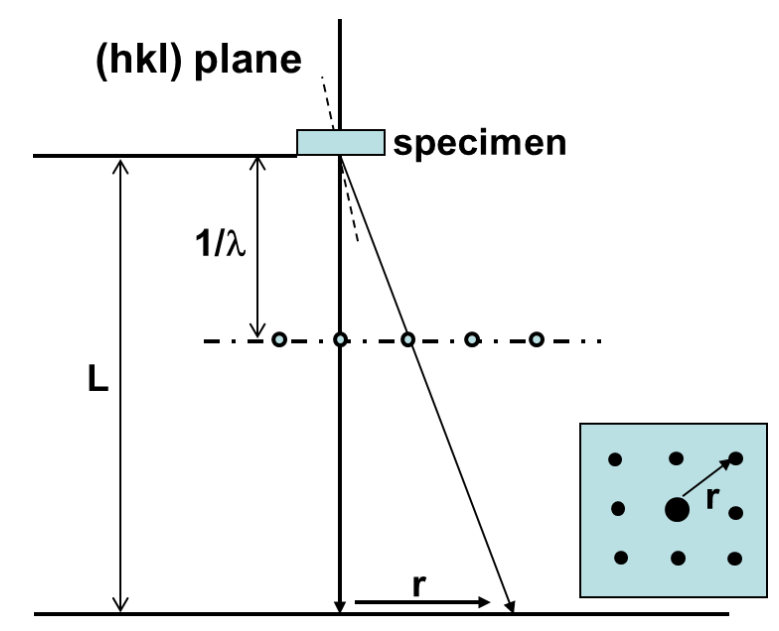

Figure 3-5 The geometric relationship between reciprocal lattice and electron diffraction pattern after [207]. 


\subsubsection{Quantitative EDS analysis}

It is known that the most straightforward method to study an unknown phase is to identify its elemental constituents. The first step is the qualitative EDS analysis. The further quantitative analysis would be meaningless if the identification of element is inaccurate. The principle of qualitative energy-dispersive X-ray analysis is simple. When the incident beams interact with the host atoms, the secondary electrons escape and leave holes in the electron shells. To stabilize the energy of atoms, electrons from outer shells transfer into the inner shells, thus the X-rays with characteristic energy are emitted from the atoms. For example in an iron atom, when the $\mathrm{K}$ shell electron is replaced by an electron from $\mathrm{L}$ shell, a $6400 \mathrm{eV} \mathrm{K}_{\alpha} \mathrm{X}$-ray is emitted from the sample, or a $704 \mathrm{eV} \mathrm{L}_{\beta} \mathrm{X}$-ray is emitted if $\mathrm{L}$ shell electron is replaced by the $\mathrm{M}$ shell electron. Namely, each element has characteristic X-ray peaks which allow them to be identified. In addition, since elements with lower atomic number have fewer filled shells, normally they have fewer X-ray peaks.

In general, it is necessary to remove Bremsstrahlung X-rays and spectral artefacts in order to obtain the integrated intensities for quantitative analysis. In so-called standardless quantitative or semi-quantitative analysis, a Gaussian fit of the elemental peaks is preformed to obtain the peak intensities. The most popular algorithm is $\mathrm{ZAF}$ where $\mathrm{Z}$ is the atomic number of the element, $\mathrm{A}$ is the absorbance coefficient and $\mathrm{F}$ is the fluorescence efficiency. The peak intensities are then converted into weight or atomic percent by sensitivity factors created by taking account of the accelerating voltage of the beam. Instead of fitting a Gaussian profile, fully standardized quantification compares the areas under the peaks to standard files which are reference spectra of the elements collected under the same conditions on the same instrument. As it requires the additional standard spectra, the fully standardized quantitative analysis is time-consuming. It is commonly considered that the advantage of this form of analysis is evident only in the situation when the peaks of the elements overlap or when quantifying the trace elements. Therefore, the quantitative analysis in this study was performed by the standardless analysis which has been proved reliable. 


\subsection{Mechanical property testing}

\subsubsection{Vickers hardness measurement}

Vickers indentation was performed to measure the hardness of samples in different conditions. Hardness tests were carried out on a CV-430AAT Manual Vickers Hardness Tester with a load of $294 \mathrm{~N}(30 \mathrm{~kg})$ and indentation time of 15 s. Each result was averaged from at least eight measurements.

It is commonly accepted that the strength is in a direct proportion to the Vickers hardness values. Therefore, the strength can be roughly predicted by the Vickers hardness results which are easier to obtain. Dieter [210] reports the conversion equation by which the yield strength can be evaluated:

$$
\sigma_{y}=\frac{H}{3}
$$

Cahoon [211] presented the expression relating the ultimate tensile strength $\sigma$ to hardness:

$$
\sigma=\frac{H}{2.9}\left(\frac{n}{0.217}\right)^{n}
$$

where $\mathrm{H}(\mathrm{MPa})$ is obtained by multiplying the Vickers hardness values by 9.807 ;

$\mathrm{n}$ is the strain hardening coefficient.

\subsubsection{Tensile testing}

Tensile testing was performed to examine the mechanical response of materials and to further study the deformation mechanisms, damage mechanisms and their relationships to the microstructure. All the tensile tests were conducted at room temperature on a Zwick Roell Z050 tensile testing machine at a constant cross head velocity corresponding to an initial strain rate of $0.002 \mathrm{~s}^{-1}$. Flat rectangular tensile samples were machined along the longitudinal direction based on ASTM standard. The dimensions of tensile test samples are given in Figure 3-6. The thickness is thinned to approximately $1.5 \mathrm{~mm}$ in order to remove the oxide surface from the preceding heat treatment. The tensile strain was recorded by an attached video 
extensometer. Two special stickers attached within the gauge portion of the tensile samples were constantly captured in the video and the distance between them was tracked in real time.

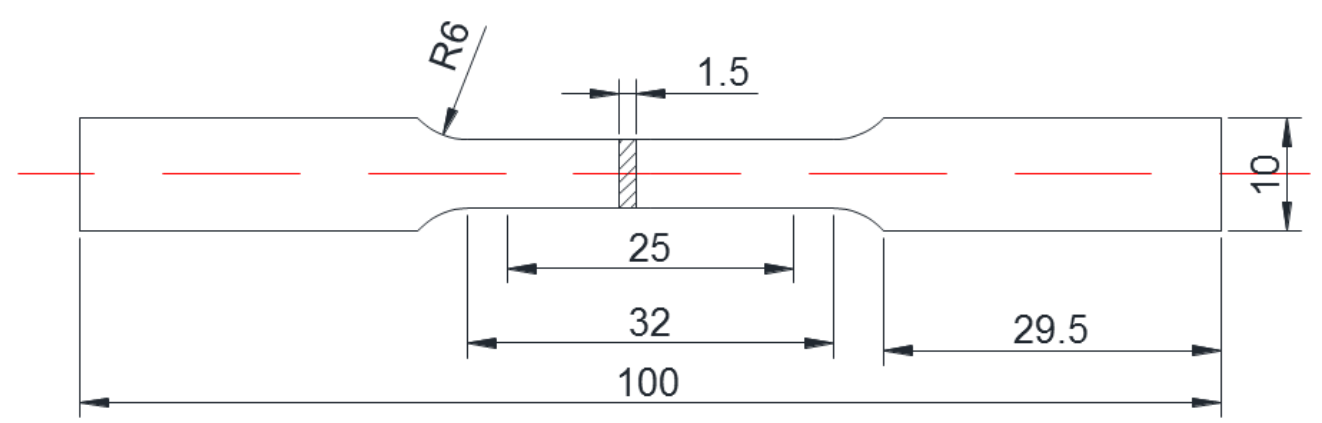

Figure 3-6 Geometry of the standard rectangular tension test sample.

\subsubsection{Nanoindentation}

As phases are deemed to have different strength, the mechanical response of different phases in the steels was examined by nanoindentation. This technique is based on indenting the selected area with a small load by an indenter tip with a specific geometry. The Berkovich tip is commonly used with the tip radius being at the range of 50-100 nm for a new indenter, see Figure 3-7(a). Since identifying the small area for indentation can be quite difficult, atomic-force microscopy (AFM) or SEM is usually used to image the microstructure and indents. Different from calculating the contact area by measuring the dimensions of indents in the conventional indentation tests, the size of residual impression in nanoindentation is on the order of nanometres and is too fine to be accurately measured even with microscopes. Therefore the depth of penetration is alternatively recorded during nanoindentation [212]. Together with the recorded force, a load-displacement curve can be obtained as shown in Figure 3-8 and different mechanical properties can therefore be evaluated: 
(a)

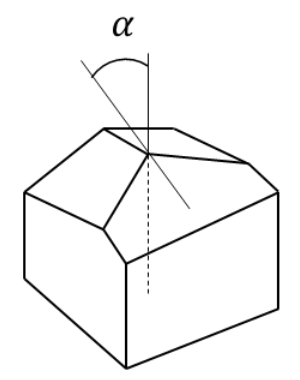

(b)

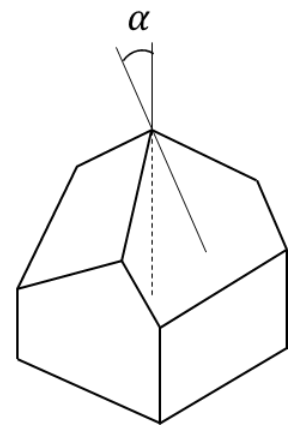

Figure 3-7 (a) a standard Berkovich indenter where $\mathrm{a}=65.03^{\circ}$; (b) a standard cube corner indenter where $\mathrm{a}=35.26^{\circ}$.

In this study, reverted austenite displays an elongated shape with the width of approximately $50-100 \mathrm{~nm}$ which is difficult to measure with conventional hardness testing. Thus nanoindentation was used to reveal the effects of aging parameters on martensite and austenite phases, respectively. In addition, the contribution of each phase to the strength can also be evaluated. For nanoindentation testing, aged samples were manually polished and etched with picric acid to reveal the microstructure under AFM. A cube corner tip (Figure 3-7(b)) was used for testing the ultrafine austenite grains. A $10 \mu \mathrm{N} / \mathrm{s}$ up to a maximum indentation load of $70 \mu \mathrm{N}$ was employed in this study.

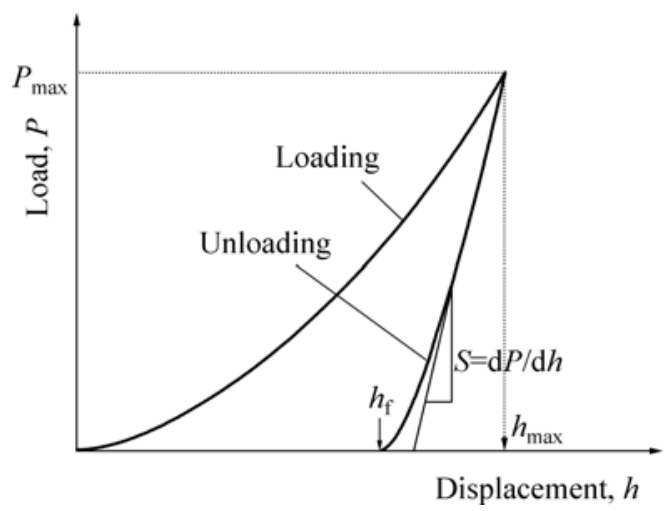

Figure 3-8 A typical schematic of load-displacement curve for nanoindentation test. 


\section{Chapter 4 Brief study on microstructure and mechanical properties of Mn-based maraging steels}

The Mn-based maraging steels studied in this work contain Ni, Ti, Al and Mo which are deemed to form intermetallic precipitates. The precipitation, which occurred during aging, is expected to increase the strength of martensite, and consequently is referred as martensite aging hardening or maraging. On the other hand, the high $\mathrm{Mn}$ content in this type of steel promotes the formation of reverted austenite during aging which is believed to affect the mechanical properties as well. In this chapter, the mechanical behaviour of Mn-based maraging steels in various conditions is evaluated by hardness and tensile testing. The mechanical differences among the three alloys are compared and analysed. The equilibrium phases as a function of temperature are predicted by MatCalc software and XRD analyses are conducted to identify the phase constitutions. Based on these studies, a general concept about the newly-developed maraging steels is presented.

\subsection{Thermodynamic calculation}

Figure 4-1 presents the equilibrium phase volume fractions as a function of temperature, which was calculated by MatCalc using MC_FE_V2.0 database. The dominant equilibrium phases at the aging temperatures of $420-500{ }^{\circ} \mathrm{C}$ are ferrite and austenite. According to MatCalc, The only precipitate phase formed in the two-phase field is Laves phase with a composition close to $\mathrm{Fe}_{3} \mathrm{Ti}(\mathrm{Mo})$. This type of precipitate forms from $\sim 800{ }^{\circ} \mathrm{C}$ and the fraction maintains between $3.45 \mathrm{vol} . \%$ to $4.42 \mathrm{vol} . \%$ at the temperature $420-500{ }^{\circ} \mathrm{C}$. Another phase which is formed above the equilibrium $\gamma / \alpha$ transformation temperature $\left(A_{e 3}\right)$ is fcc TiC. This carbide is thermally stable over a large temperature range up to $1300^{\circ} \mathrm{C}$. 
Chapter 4 Brief study on microstructure and mechanical properties of Mn-based maraging steels
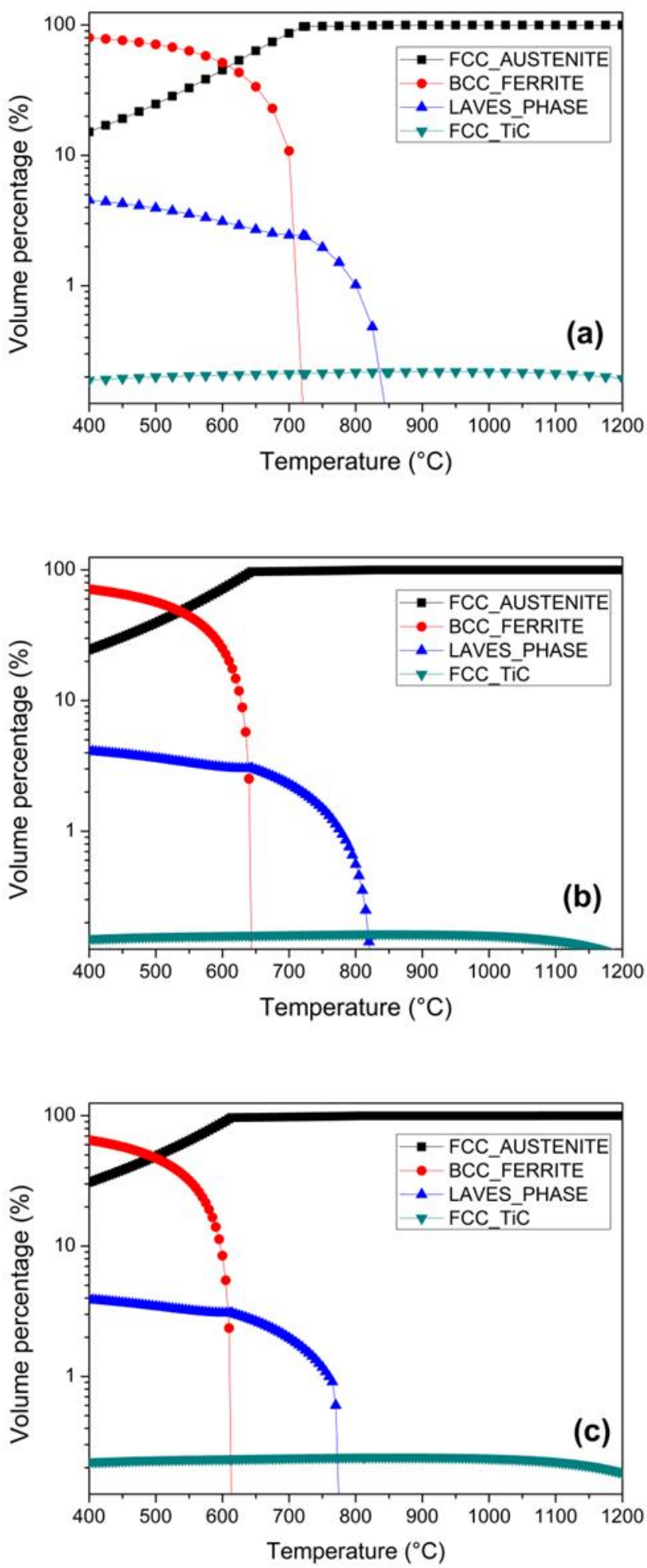

Figure 4-1 The equilibrium phase fractions as a function of temperature in (a) $7 \% \mathrm{Mn}$, (b) $10 \%$ Mn and (c) $12 \%$ Mn alloys. 


\subsection{The effect of Mn content on grain refinement}

The grain size distribution of $7 \%, 10 \%$ and $12 \% \mathrm{Mn}$ alloys in the SHT state was characterized by EBSD and the results are displayed in Figure 4-2. The left column presents the martensite phase (blue) with grain boundaries (black lines, $>15^{\circ}$ ). The EBSD phase maps show that in the solution-treated condition, only martensite was present. This result disagrees with the EBSD observation by Raabe et al. [18] suggesting that a small amount of austenite was retained after quenching in $12 \mathrm{wt} . \%$ Mn alloy. Apart from the EBSD resolution limit, there are three possible reasons about this deviation. (i) Raabe may have used a slower cooling rate by which some austenite was retained rather than transformed to $\alpha^{\prime}$-martensite. (ii) Both experiment and model simulation demonstrated the dependence of the martensite-start temperature $\left(M_{s}\right)$ on the prior austenite grain size [34][162][213]. It is reported the ultrafine prior austenite grain is one of the reason for the presence of retained austenite. The small grain size suppresses the martensitic transformation, and thereby contributing to the austenite stability during cooling [34][213]. (iii) The addition of $\mathrm{Al}$ in the present study increased $\mathrm{M}_{\mathrm{s}}$ temperature.

The right column presents the inverse pole coloured orientation maps of the same area, in which the packets of lath martensite can be clearly observed. It is evident that these martensite packets formed via water quenching possessed high-angle misorientation with adjacent packets. Each prior austenite grain was sub-divided into several martensite packets. In other words, the effective grain size was refined owing to the dense high-angle boundaries induced by martensite packets. In addition, it is noticed that the effective grain size was reduced as the Mn content of the alloy increased, which means that the Mn addition contributed to the grain refinement in these alloys. It is known that the addition of $\mathrm{Mn}$ lowers both the $A_{e 3}$ and $M_{S}$ temperatures. The reduction of $A_{e 3}$ provides an increased driving force for phase transformation and thus a decreased driving force for grain growth. Similarly, a lower $M_{S}$ temperature leads to the refined packets of lath martensite. Therefore, finer prior austenite grains and martensite packets were observed in $12 \% \mathrm{Mn}$ alloy in the SHT state compared to other two alloys. 
Chapter 4 Brief study on microstructure and mechanical properties of Mn-based maraging steels
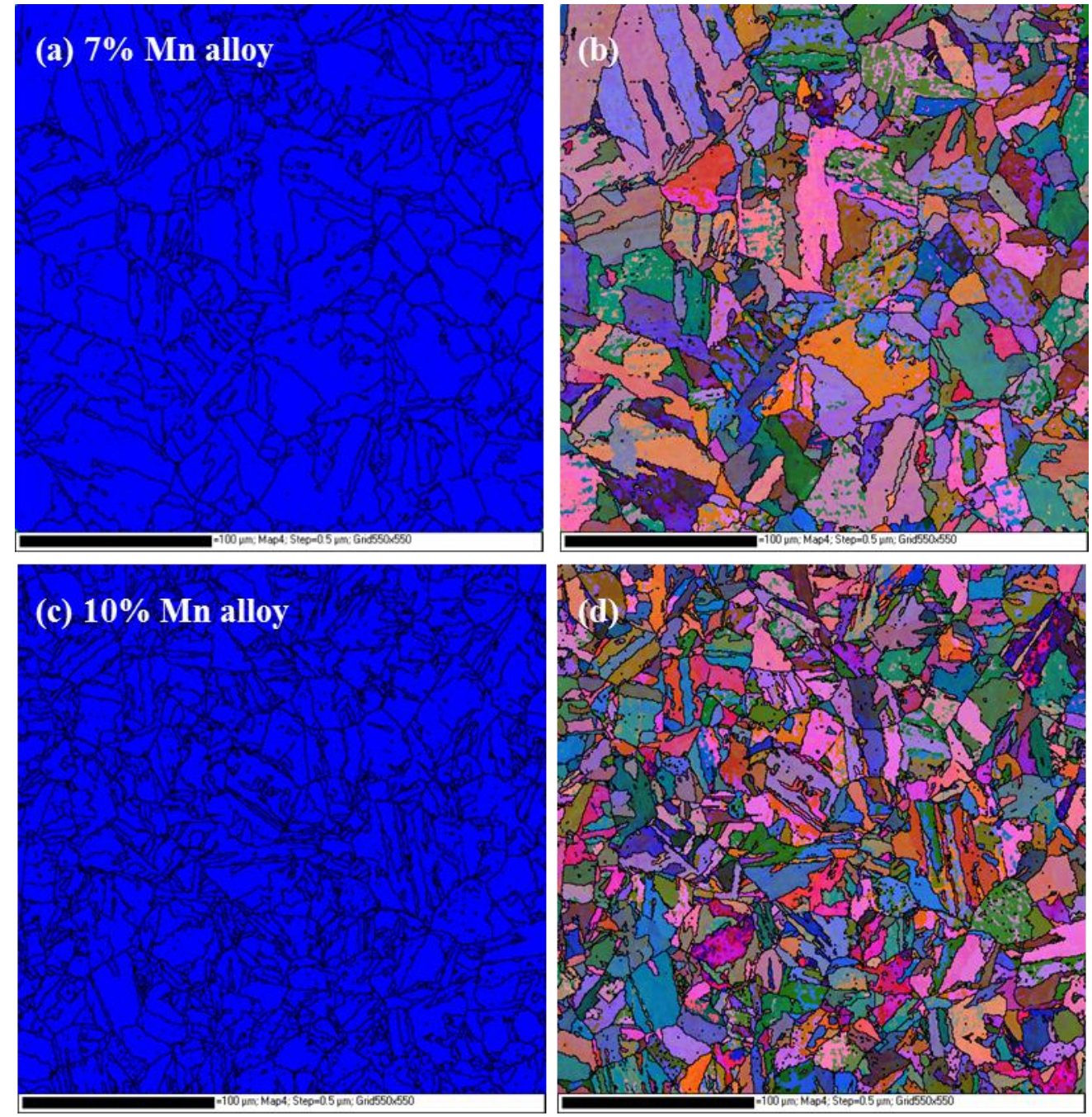

(e) $12 \% \mathrm{Mn}$ alloy

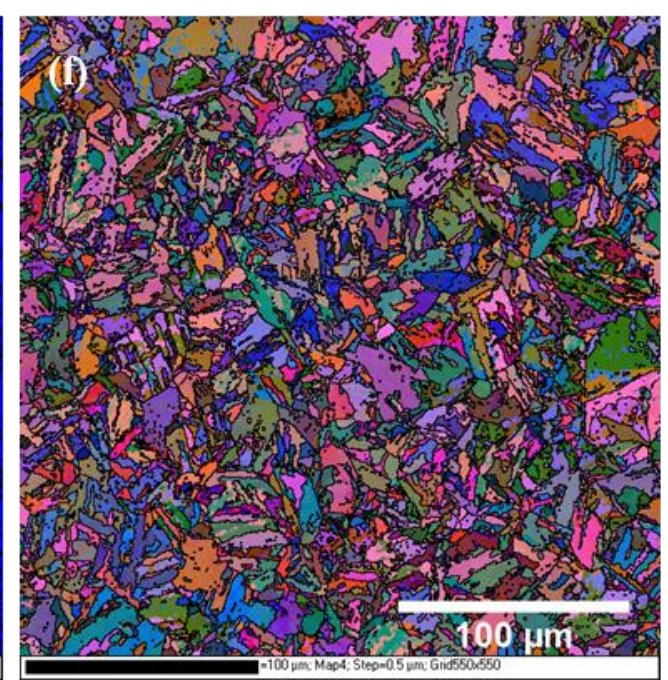

Figure 4-2 Microstructure of the three alloys in the SHT state by EBSD analyses. Left column: phase maps showing martensite phase (blue) with grain boundaries (black lines, $>15^{\circ}$ ). Right column: inverse pole maps of the same area, showing the orientation distribution of the martensite. 


\subsection{Phase identification by XRD and SEM}

X-ray diffraction (XRD) analyses were conducted on all the solution-treated and aged samples. Figure 4-3 displays the XRD results of the three alloys in the SHT state, which confirms only martensite could be detected in the three samples.

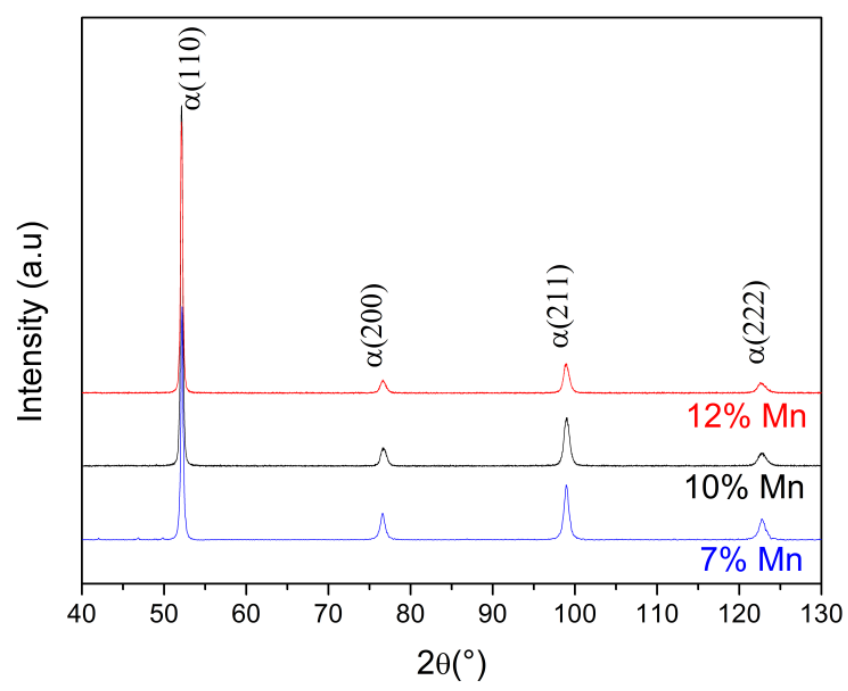

Figure 4-3 X-ray diffraction patterns of solution-treated 7\%, 10\% and 12\% Mn alloys.

Although both XRD and EBSD analyses did not show any evidence of carbide formation, the SEM micrographs in Figure 4-4(a) and (b) reveals the existence of carbide in the SHT state. The SEM-EDS and TEM-EDS analyses (Figure 4-4(b) and (c)) confirm that this type of carbide is Ti-rich as MatCalc predicts. It is shown in Figure 4-4(a) that both the size and the number density of $\mathrm{TiC}$ particles are small (0.15-0.22 vol.\% provided by MatCalc) and this is the reason why the XRD and EBSD failed to detect carbide. In addition, MatCalc indicated this kind of carbide is very stable and cannot be totally dissolved even heating up to $1300{ }^{\circ} \mathrm{C}$, so in this work the carbides were retained and could be observed in both SHT and aged samples (Figure 4-4(d)). 

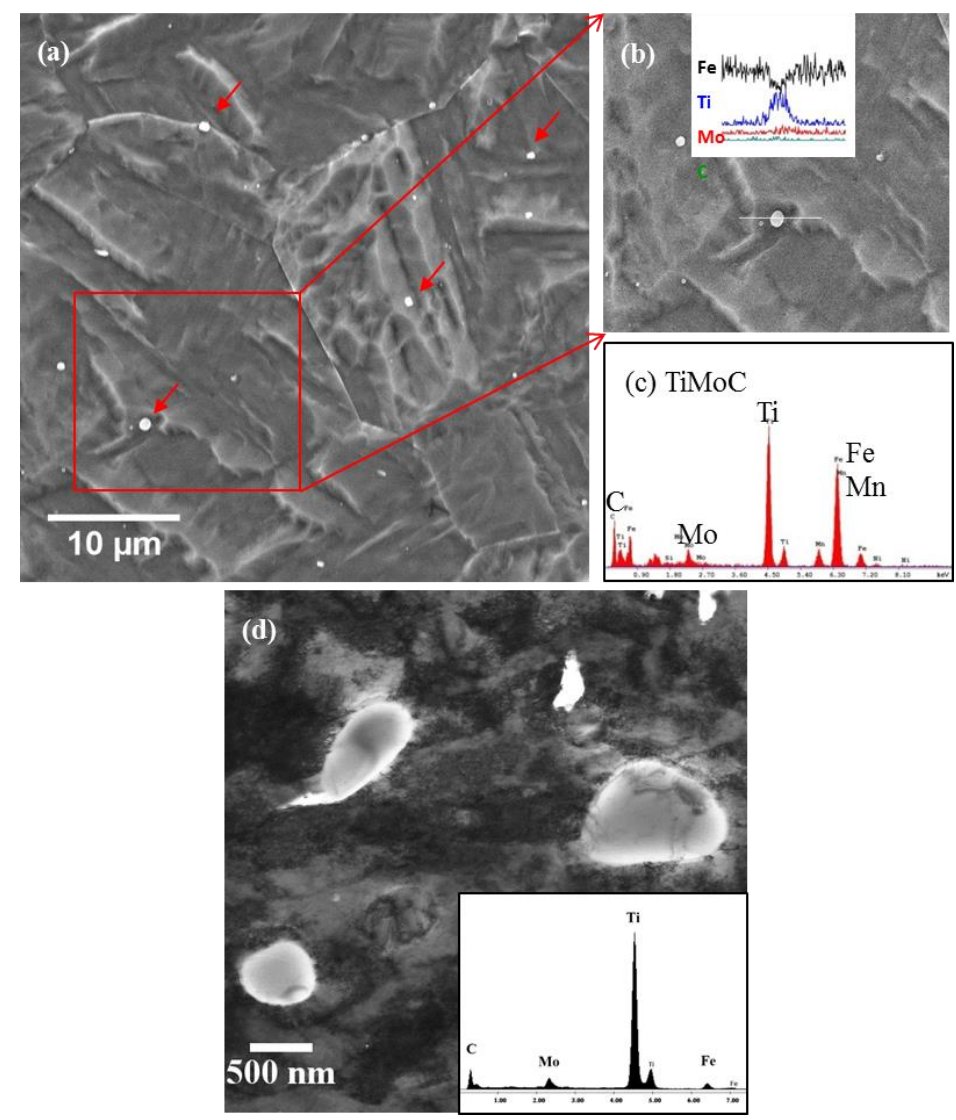

Figure 4-4 (a) SEM micrograph of Ti-rich carbide present in 10\% Mn alloy in the SHT state with (b) EDS line scan and (c) EDS spot detection results; (d) TEM micrograph of Ti-rich carbide existing in aged $7 \% \mathrm{Mn}$ alloy in $500{ }^{\circ} \mathrm{C} / 10080 \mathrm{~min}$ state.

Further aging treatment is supposed to lead to the precipitation and the formation of reverted austenite. However, the X-ray diffraction patterns of most aged samples only reveal the existence of martensite. Distinguishable austenite peaks were mainly observed in $10 \%$ and $12 \%$ Mn alloys in the overaged stage at $500{ }^{\circ} \mathrm{C}$, see Figure 4-5. At $460{ }^{\circ} \mathrm{C}$, the presence of austenite was only observed after long-term aging (not presented), while at $420{ }^{\circ} \mathrm{C}$, there was no evidence of the formation of reverted austenite in the three alloys (not presented). 

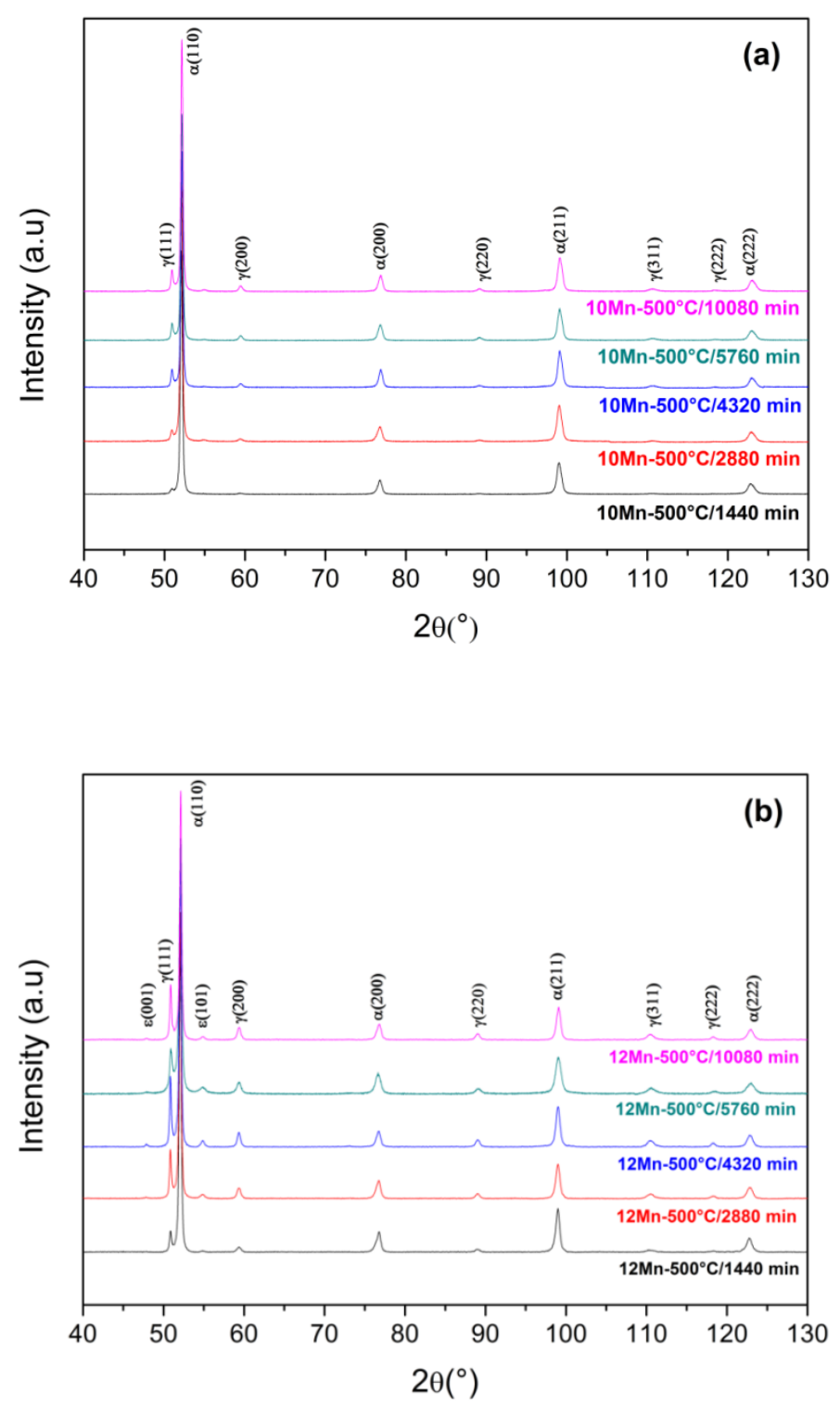

Figure 4-5 X-ray diffraction patterns of (a) $10 \% \mathrm{Mn}$ and (b) $12 \% \mathrm{Mn}$ alloys aged at $500{ }^{\circ} \mathrm{C}$ for various durations.

Apart from peaks of martensite and austenite, additional peaks were noticed in the samples aged at $500{ }^{\circ} \mathrm{C}$ for a long duration (Figure 4-5), which were later demonstrated to be $\varepsilon$-martensite phase. The $\varepsilon$-martensitic transformation upon cooling has been interpreted by Schumann in his study on Fe-Mn steels [214]. The Schumann's martensitic transformation diagrams are displayed in Figure 4-6 [154][215]. According to Schumann, the martensitic transformation in Fe-Mn steels 
can be summaries as: (i) when the Mn concentration of austenite is below $10 \mathrm{wt} \%$, $\gamma \rightarrow \alpha^{\prime}$ martensitic transformation occurs during cooling and only $\alpha^{\prime}$ martensite can be obtained at room temperature, (ii) when the Mn concentration is between $10 \mathrm{wt} . \%$ and $15 \mathrm{wt} . \%$, the $M_{s}$ temperatures of $\gamma \rightarrow \alpha^{\prime}$ and $\gamma \rightarrow \varepsilon$ transformations are close (Figure 4-6(a)). Although part of the $\varepsilon$-martensite may still transform into $\alpha^{\prime}$ martensite, the presence of both $\alpha^{\prime}$ - and $\varepsilon$-martensite along with untransformed $\gamma$ phase is expected after cooling, (iii) when the Mn concentration is between 15 wt.\% and $25 \mathrm{wt} . \%$, the thermally stable phases at room temperature are $\varepsilon$-martensite and $\gamma$ phase which is retained due to fast cooling rate, (iv) when the Mn concentration is over $\sim 25$ wt. $\%$, both the $M_{s}$ temperatures of $\gamma \rightarrow \alpha^{\prime}$ and $\gamma \rightarrow \varepsilon$ transformations have been reduced to below room temperature. In theory the alloy at room temperature should be constituted by fully austenite content, but some studies reported a trace of $\varepsilon$-martensite in alloys with Mn concentration higher than 25 wt.\% [216]. In addition, it has been demonstrated that the $\gamma \rightarrow \varepsilon$ martensitic transformations is controlled by a displacement mechanism and thus there is no change in the concentration after phase transformation [214].
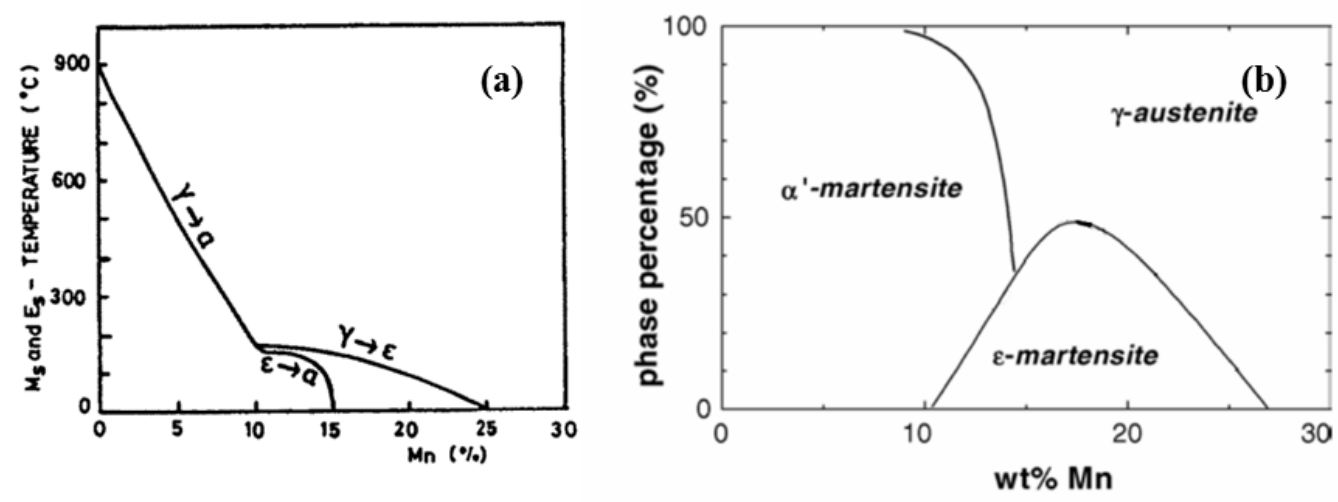

Figure 4-6 (a) Schumann's martensitic transformation diagram of the Fe-Mn system [154];

(b) schematic map of the phase fractions after cooling to room temperature based on Schumann's description [215].

It is worth noting that no peaks corresponding to precipitates were detected by XRD. But a large number of uniformly distributed precipitates were noticed in aged samples under microscope observation, as shown in Figure 4-7. The elongated 
islands with brighter contrast are the reverted austenite phase, whereas the nanoscaled spherical phases are the precipitates. A possible explanation is that the diffraction peaks of precipitates are overlapped by those of $\alpha^{\prime}$-martensite matrix. A more detailed study on precipitates will be given in Chapter 5 .
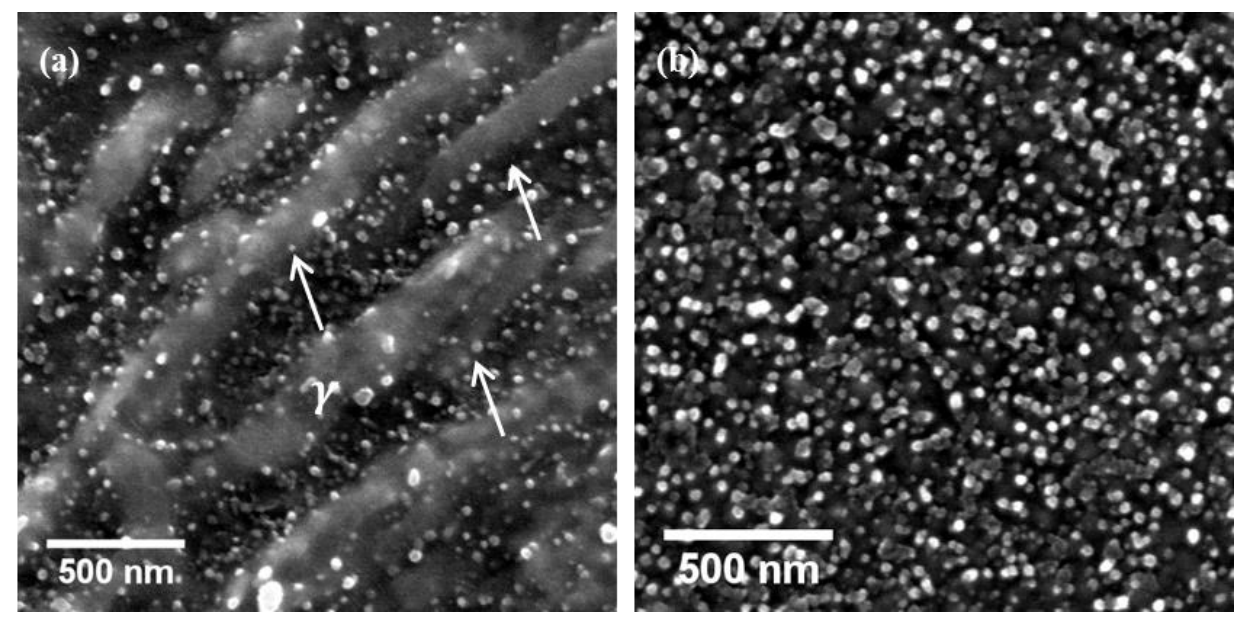

Figure 4-7 SEM micrographs of (a) lath-like austenite and precipitate-decorated martensite matrix and (b) homogenously distributed precipitates in $12 \% \mathrm{Mn}$ alloy in $500{ }^{\circ} \mathrm{C} / 2880 \mathrm{~min}$ state.

\subsection{Hardness evolution}

The hardness curves of precipitation-strengthened alloys generally exhibit four regions: (i) incubation; (ii) a rapid increase in hardness (under-aged region); (iii) a plateau of hardness (peak-aged point) and (iv) a decrease in hardness (overaged region). Figure 4-8 displays the hardness evolution of 7\%, 10\% and 12\% Mn alloys aged at different temperatures.

It is apparent that aging at $420^{\circ} \mathrm{C}$ for a limited time up to 10080 minutes did not lead to the peak hardness in the three alloys. As presented in Figure 4-8(a), the three alloys experienced a long incubation and under-aging process which indicated a very slow hardening kinetics in Mn-based maraging steels when aging at $420{ }^{\circ} \mathrm{C}$. It should be noted that higher hardness of alloys with higher Mn content (i.e. $H_{12 \% M n}>H_{10 \% M n}>H_{7 \% M n}$ ) can be observed for each state (Figure 4-8(a)).

Aging at $460{ }^{\circ} \mathrm{C}$ led to the peak hardness in $10 \% \mathrm{Mn}$ and $12 \% \mathrm{Mn}$ alloys (Figure 4-8(b)). Although the over-aged region was not that evident in 7\% Mn alloy, a 
decreasing slope of hardness curve leading to a plateau indicates that the peak hardness of $7 \% \mathrm{Mn}$ alloy aged at $460^{\circ} \mathrm{C}$ was achieved within 10080 minutes as well. As expected, $12 \% \mathrm{Mn}$ alloy was the first who reached the maximum hardness (453 $\mathrm{HV}, 480 \mathrm{~min}$ ), followed by 10\% Mn alloy approaching the peak (457 HV, $1440 \mathrm{~min}$ ) and then 7\% Mn alloy (454 HV, $5760 \mathrm{~min}$ ).

Figure 4-8(c) displays the hardness curves of the three alloys aged at $500{ }^{\circ} \mathrm{C}$, which exhibit a typical hardness evolution of precipitation-strengthened alloys. A very rapid increase of hardness was visible in the early aging region of the 10\% Mn and $12 \% \mathrm{Mn}$ alloys, over $90 \%$ of the total increase in hardness was achieved within the first 30 minutes. While an incubation in the first 30 minutes was evident in $7 \% \mathrm{Mn}$ alloy followed by a sluggish hardening process leading to the hardness peak. On the other hand, the maximum hardness of the three alloys are very close (7\% Mn alloy: $418 \mathrm{HV}$ at $240 \mathrm{~min} ; 10 \% \mathrm{Mn}$ alloy: $421 \mathrm{HV}$ at $240 \mathrm{~min} ; 12 \% \mathrm{Mn}$ alloy: $432 \mathrm{HV}$ at $120 \mathrm{~min})$. It is worth noting that in the over-aged region, what appears to be secondary hardening was observed in both $10 \% \mathrm{Mn}$ and $12 \% \mathrm{Mn}$ alloy. Actually, a less evident secondary hardening in the overaged stage could also be observed in the two alloys at $460{ }^{\circ} \mathrm{C}$. This secondary hardening indicates there might be a new phase formed during this period. 
Chapter 4 Brief study on microstructure and mechanical properties of Mn-based maraging steels
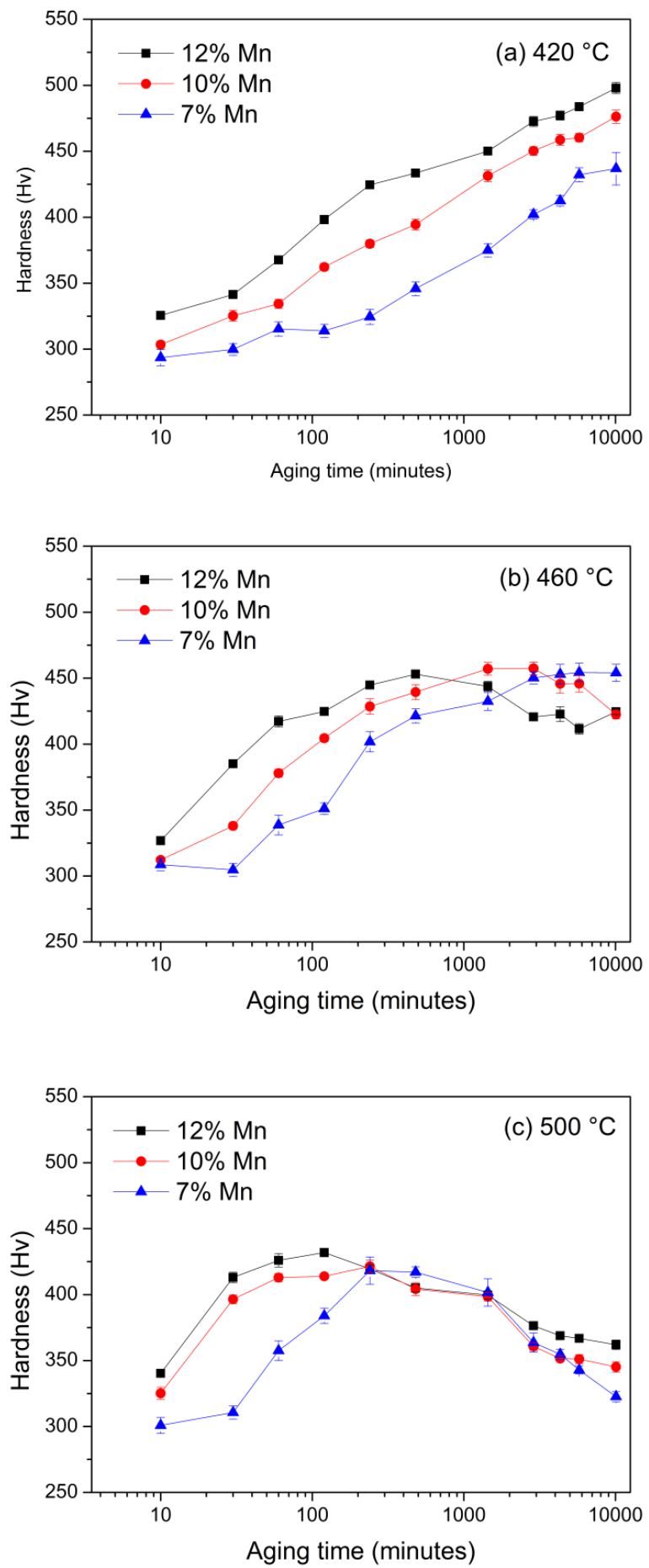

Figure 4-8 Vickers hardness as a function of aging time at (a) $420{ }^{\circ} \mathrm{C}$, (b) $460{ }^{\circ} \mathrm{C}$ and (c) $500{ }^{\circ} \mathrm{C}$ for Mn-based maraging steels, respectively. 


\subsection{Tensile results}

\subsubsection{Tensile behaviour of solution-treated samples}

Figure 4-9 displays the engineering stress-strain curves of $7 \%, 10 \%$ and $12 \% \mathrm{Mn}$ alloys in the SHT state and the summarized data are listed in Table 4-1. It is obvious that the yield strength (YS), ultimate tensile strength (UTS) and tensile elongation (TE) increase with the Mn content of alloys, but the differences among the three alloys are small. It is believed that the more prominent solid solution strengthening owing to higher Mn content is responsible for the higher UTS and TE of 12\% Mn alloy. In addition, the Mn addition leading to the refinement of both prior austenite grains and martensite laths which contributes to the improvement of strength and ductility. It should be noted that after reaching the yield point, the increase of engineering stress was small. Instead, an evident decrease of engineering stress as a function of strain was observed. This indicates that the Mn-based maraging steels in the SHT state did not possess a good work hardening during deformation.

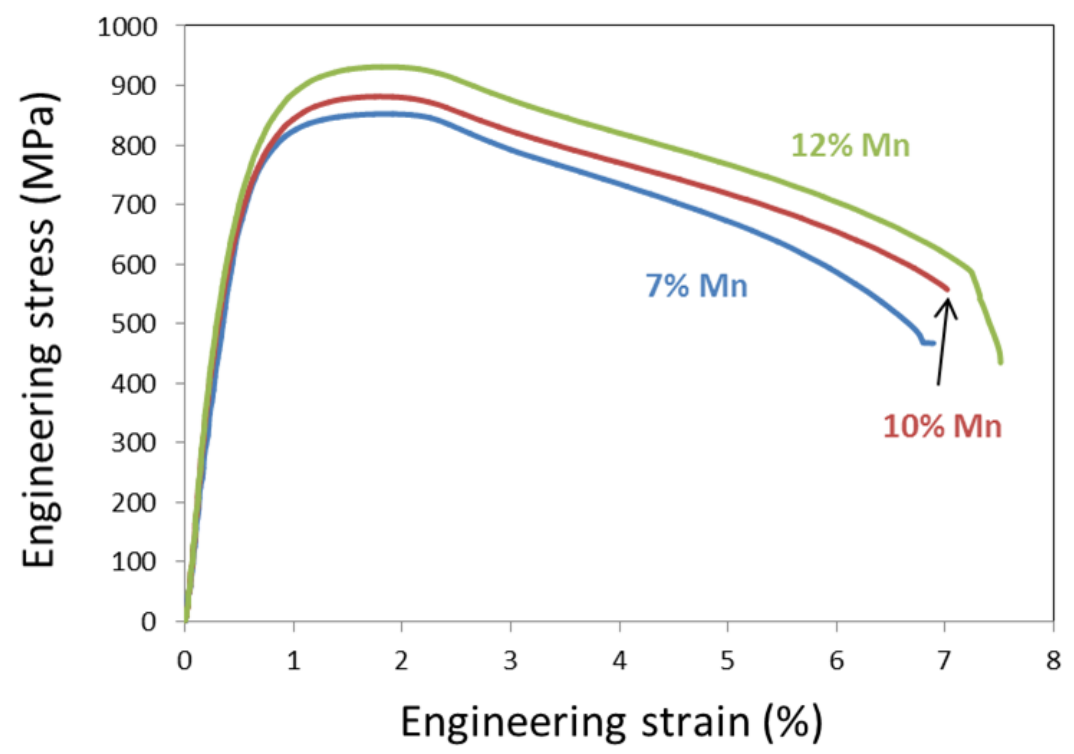

Figure 4-9 Engineering stress vs. strain of 7\%, 10\% and 12\% Mn alloys in the SHT state. 
Chapter 4 Brief study on microstructure and mechanical properties of Mn-based maraging steels

Table 4-1 Summary of the mechanical properties of 7\% Mn, 10\% Mn and 12\% Mn alloys in the SHT state.

\begin{tabular}{cccc}
\hline & YS (MPa) & UTS (MPa) & TE (\%) \\
\hline $7 \% \mathrm{Mn}$ & 770 & 852 & 6.9 \\
$10 \% \mathrm{Mn}$ & 790 & 882 & 7.1 \\
$12 \% \mathrm{Mn}$ & 820 & 931 & 7.5 \\
\hline
\end{tabular}

\subsubsection{The embrittlement of aged samples}

After aging, the three alloys suffered from poor ductility at lower aging temperature or at higher temperature but for short time. Most of them failed before approaching the yield point (see Figure 4-10(a)). The images of the fracture surface reveal that there was no visible necking (reduction of area), and cracks along grain boundaries can be clearly observed (Figure 4-10(b) and (c)). Aging at higher temperatures or for longer times were demonstrated to significantly improve the embrittlement. Besides, the Mn content of alloy was also found to affect the ductility. In the following section, the tensile behaviour of Mn-based maraging steels will be studied in terms of the aging time, aging temperature and the Mn content, respectively. 
Chapter 4 Brief study on microstructure and mechanical properties of Mn-based maraging steels
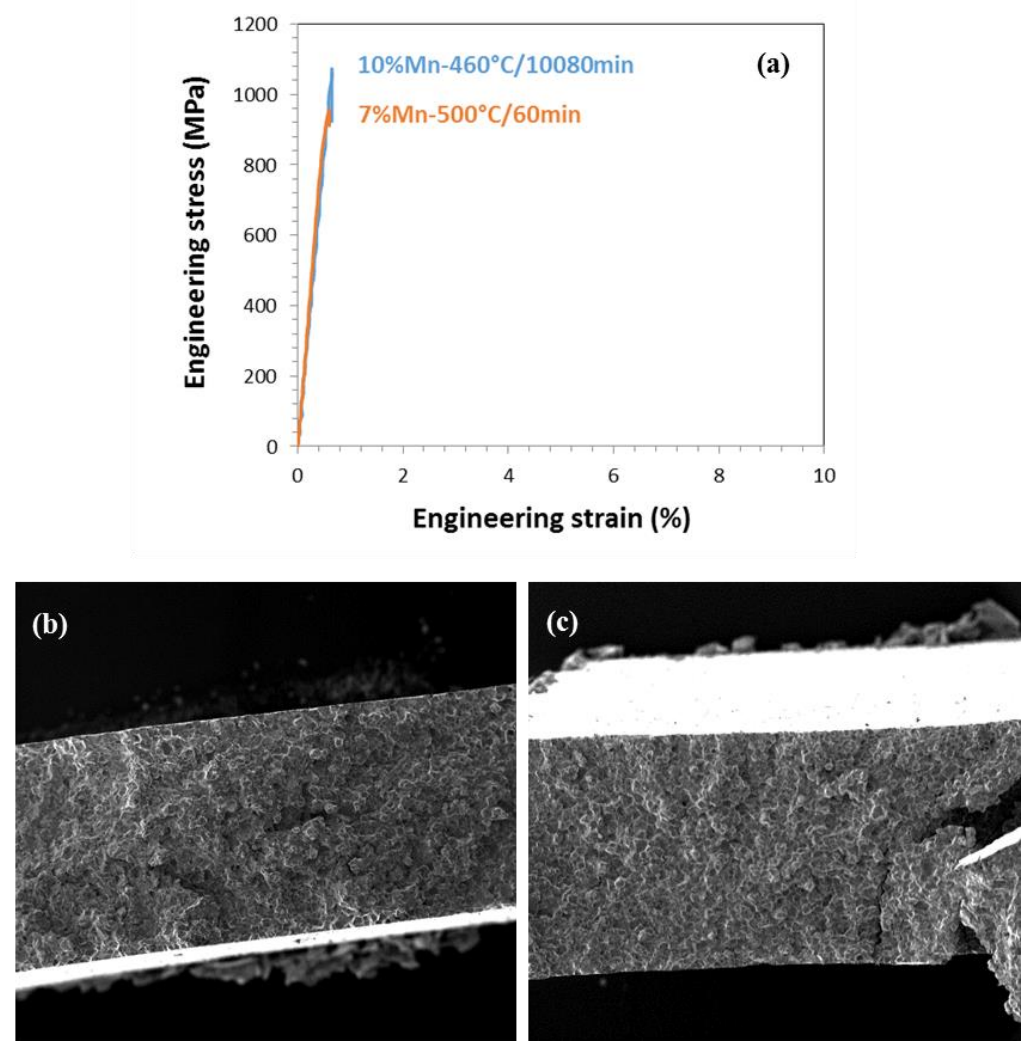

Figure 4-10 (a) Engineering stress-strain curves of $10 \% \mathrm{Mn}$ alloy in $460{ }^{\circ} \mathrm{C} / 10080 \mathrm{~min}$ state and $7 \% \mathrm{Mn}$ alloy in $500{ }^{\circ} \mathrm{C} / 60 \mathrm{~min}$ state with their corresponding fracture images: (b) $10 \% \mathrm{Mn}$ alloy in $460{ }^{\circ} \mathrm{C} / 10080 \mathrm{~min}$ state and (c) $7 \% \mathrm{Mn}$ alloy in $500{ }^{\circ} \mathrm{C} / 60 \mathrm{~min}$ state.

\subsubsection{The improvement of mechanical properties in overaged samples}

The engineering stress-strain curves of $10 \% \mathrm{Mn}$ alloys aged at $500{ }^{\circ} \mathrm{C}$ where the plastic deformation was clearly present are displayed in Figure 4-11. The samples showing early embrittlement are not displayed here. After aging at $500{ }^{\circ} \mathrm{C}$ for over 2880 minutes, both UTS and TE were considerably improved. The UTS was increased from $832 \mathrm{MPa}$ in the SHT state to $1113 \mathrm{MPa}$ in $500{ }^{\circ} \mathrm{C} / 2880 \mathrm{~min}$ state, whereas the TE increased from $7.0 \%$ to $12.1 \%$. Further aging for 5760 minutes led to the TE gradually increasing up to $17.0 \%$ but the UTS slightly reducing to $1062 \mathrm{MPa}$. Aging up to 10080 minutes unexpectedly resulted in a decrease in TE to $13.7 \%$, whereas the UTS stayed at the same level. A summary of YS, UTS and TE are listed 
Chapter 4 Brief study on microstructure and mechanical properties of Mn-based maraging steels

in Table 4-2. It is worth noting that, compared to the engineering stress-strain curves of the solution-treated samples, the engineering stress of aged samples in Figure 4-11 reached a plateau after the yield point and stayed at the plateau over a wide strain range.

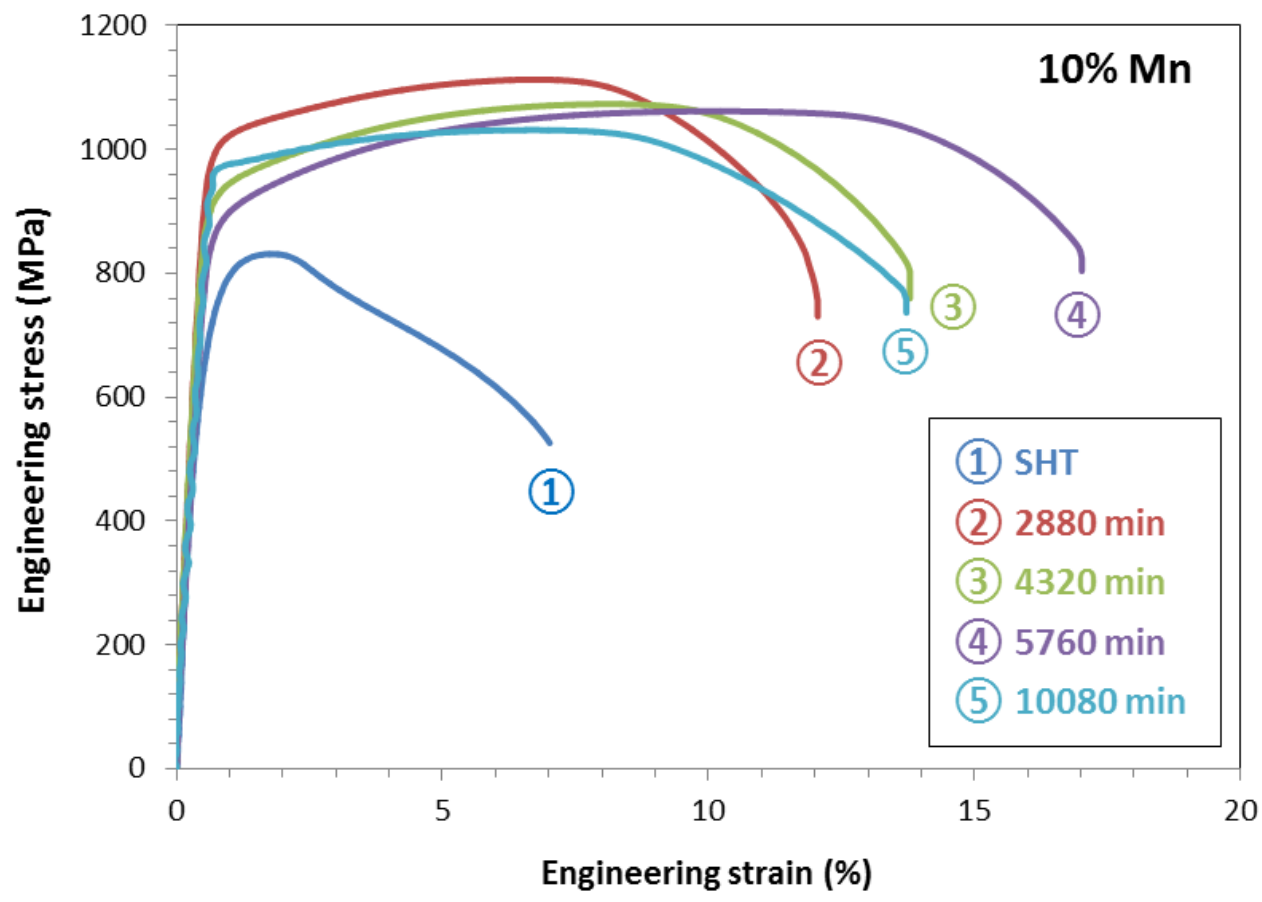

Figure 4-11 Engineering stress vs. engineering strain of $10 \% \mathrm{Mn}$ alloy aged at $500{ }^{\circ} \mathrm{C}$ for different durations.

Table 4-2 Summary of the mechanical properties of $10 \% \mathrm{Mn}$ alloys aged at $500{ }^{\circ} \mathrm{C}$ for different durations.

\begin{tabular}{cccc}
\hline & YS (MPa) & UTS (MPa) & TE (\%) \\
\hline 2880 min & 1010 & 1113 & 12.1 \\
$4320 \mathrm{~min}$ & 920 & 1074 & 13.8 \\
$5760 \mathrm{~min}$ & 860 & 1062 & 17.0 \\
$10080 \mathrm{~min}$ & 970 & 1032 & 13.7 \\
\hline
\end{tabular}


Similarly in the $12 \% \mathrm{Mn}$ alloy aged at $500{ }^{\circ} \mathrm{C}$, the aging led to the increase in strength but uncertain changes in ductility (Figure 4-12 and Table 4-3). In the early aging stage, samples failed without exhibiting any ductility (not displayed). After aging for $240 \mathrm{~min}$, an improved TE to $5.5 \%$ with a remarkable UTS of $1306 \mathrm{MPa}$ was noticed. Longer aging improved the ductility but was found detrimental to the strength (e.g. the sample in $500{ }^{\circ} \mathrm{C} / 2880 \mathrm{~min}$ state: UTS of $1142 \mathrm{MPa}$, TE of $17.8 \%$ ). However, further extended aging did not result in any obvious variation in the mechanical properties (4) (5) (6) (7) in Figure 4-12). What is worth emphasising is that similar but more flattened engineering stress-strain curves than $10 \% \mathrm{Mn}$ alloy was present in $12 \% \mathrm{Mn}$ alloy. It is evident that the decrease of engineering stress during the necking period was smaller than that in $12 \% \mathrm{Mn}$ alloy for the same conditions.

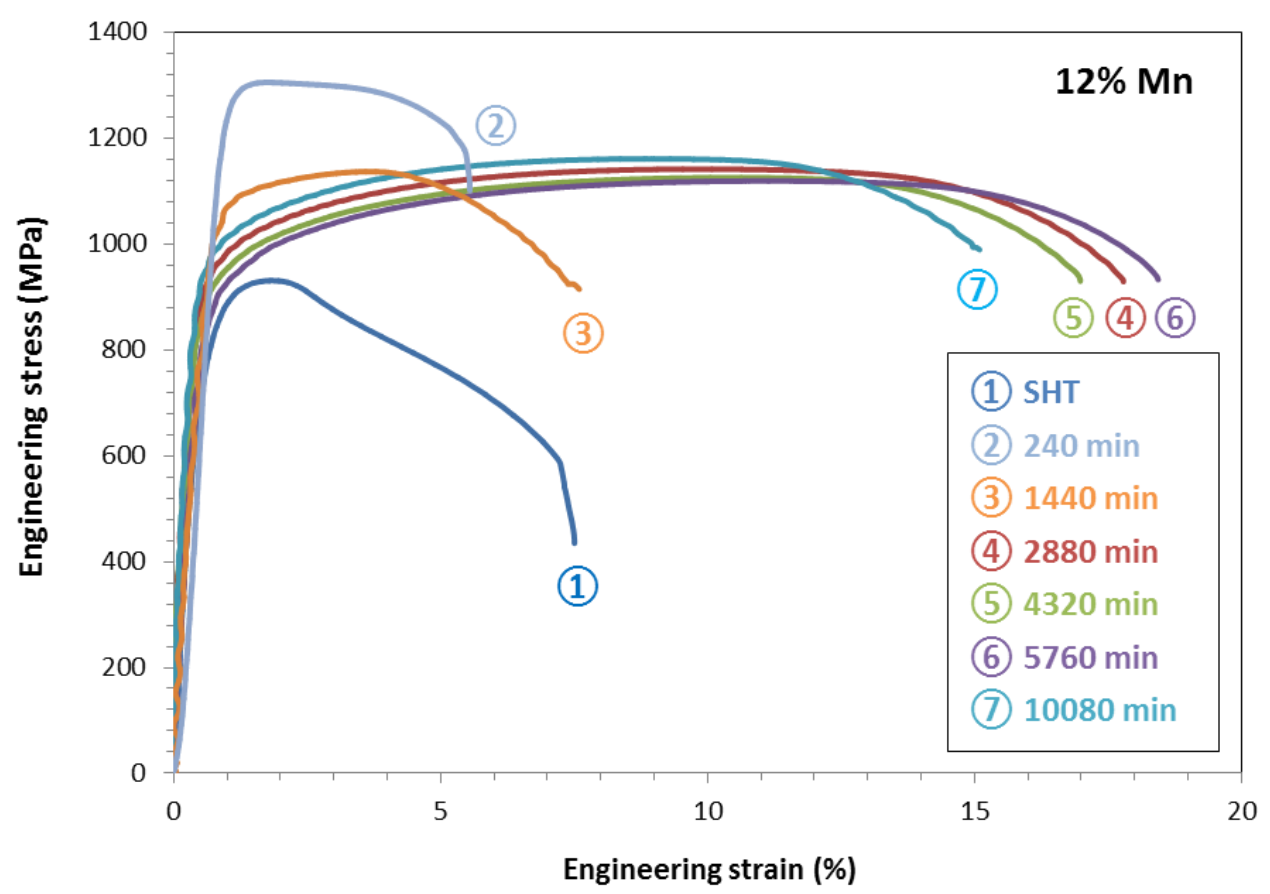

Figure 4-12 Engineering stress vs. strain of $12 \% \mathrm{Mn}$ alloy aged at $500{ }^{\circ} \mathrm{C}$ for different durations. 
Chapter 4 Brief study on microstructure and mechanical properties of Mn-based maraging steels

Table 4-3 Summary of mechanical properties of $12 \% \mathrm{Mn}$ alloy aged at $500{ }^{\circ} \mathrm{C}$ for different durations.

\begin{tabular}{cccc}
\hline & YS (MPa) & UTS (MPa) & TE (\%) \\
\hline 240 min & 1250 & 1306 & 5.5 \\
$1440 \mathrm{~min}$ & 1015 & 1137 & 7.6 \\
$2880 \mathrm{~min}$ & 940 & 1142 & 17.8 \\
$4320 \mathrm{~min}$ & 910 & 1126 & 17.0 \\
$5760 \mathrm{~min}$ & 870 & 1120 & 18.4 \\
$10080 \mathrm{~min}$ & 960 & 1162 & 15.1 \\
\hline
\end{tabular}

In terms of the effects of aging temperature, all the three alloys exhibited poor ductility when aging at $420{ }^{\circ} \mathrm{C}$ even for 10080 minutes (Figure 4-13 exhibits the tensile curve of $12 \% \mathrm{Mn}$ alloy in $420{ }^{\circ} \mathrm{C} / 10080 \mathrm{~min}$ state). Increasing aging temperature to $460{ }^{\circ} \mathrm{C}$ improved the embrittlement. The $12 \% \mathrm{Mn}$ alloy in $460{ }^{\circ} \mathrm{C}$ / 10080 min state attained a limited TE of $7.7 \%$ with a UTS of $1330 \mathrm{MPa}$. Significantly improved ductility but decreased strength existed when aging at $500{ }^{\circ} \mathrm{C}$.

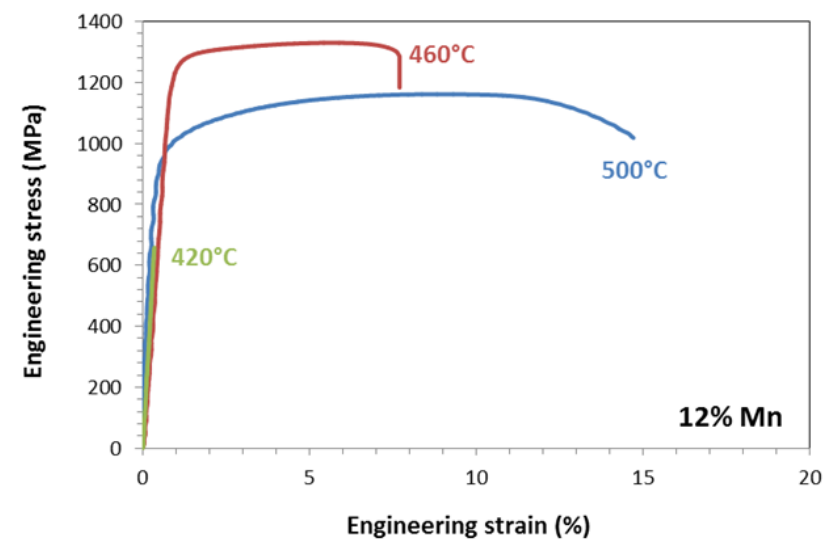

Figure 4-13 Engineering stress-strain curves of 12\% Mn alloy aged for 10080 minutes at different temperatures.

Increasing the Mn content of alloy is considered to another effective method to improve both the strength and ductility. As stated above, at lower aging temperatures 
$\left(420{ }^{\circ} \mathrm{C}\right.$ and $460{ }^{\circ} \mathrm{C}$ ), $7 \% \mathrm{Mn}$ and $10 \% \mathrm{Mn}$ alloy failed before plastic deformation initiated. Only the $12 \% \mathrm{Mn}$ alloy in $460{ }^{\circ} \mathrm{C} / 10080 \mathrm{~min}$ state exhibited an acceptable combination of strength and ductility (Figure 4-13). At the aging temperature of $500{ }^{\circ} \mathrm{C}$ where the embrittlement was supposed to be much improved, 7\% $\mathrm{Mn}$ alloy in $500{ }^{\circ} \mathrm{C} / 10080$ state yielded a TE of $6.6 \%$, while both the $10 \% \mathrm{Mn}$ and $12 \% \mathrm{Mn}$ alloys yielded even better ductility (13.7\% and 15.3\%, respectively). Moreover, the UTS was also increased from $1005 \mathrm{MPa}$ of $7 \% \mathrm{Mn}$ alloy to $1162 \mathrm{MPa}$ of $12 \% \mathrm{Mn}$ alloy (Figure 4-14). Figure 4-15 further compares the UTS and TE between 10\% Mn and $12 \% \mathrm{Mn}$ alloys aged at $500{ }^{\circ} \mathrm{C}$ for different durations, which reveals that both the UTS and TE of 12\% Mn alloy were higher than those of 10\% Mn alloy for the same conditions.

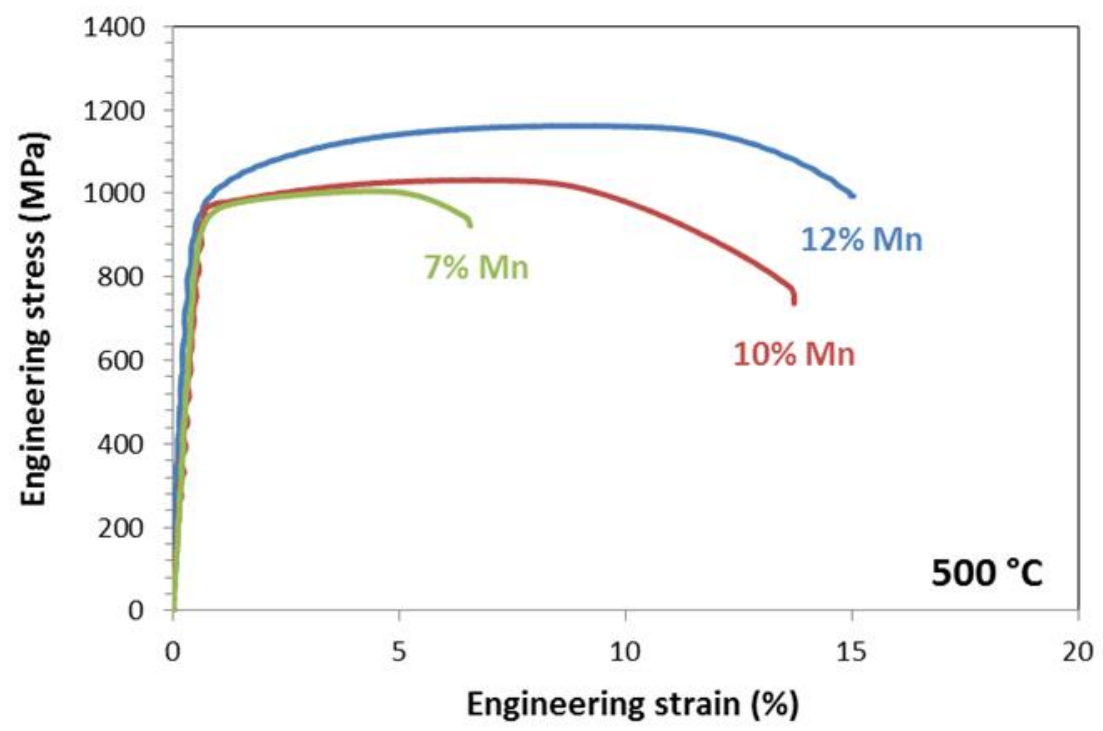

Figure 4-14 Engineering stress-strain curves of 7\% Mn, 10\% Mn and 12\% Mn alloys in $500{ }^{\circ} \mathrm{C} / 10080 \mathrm{~min}$ state. 


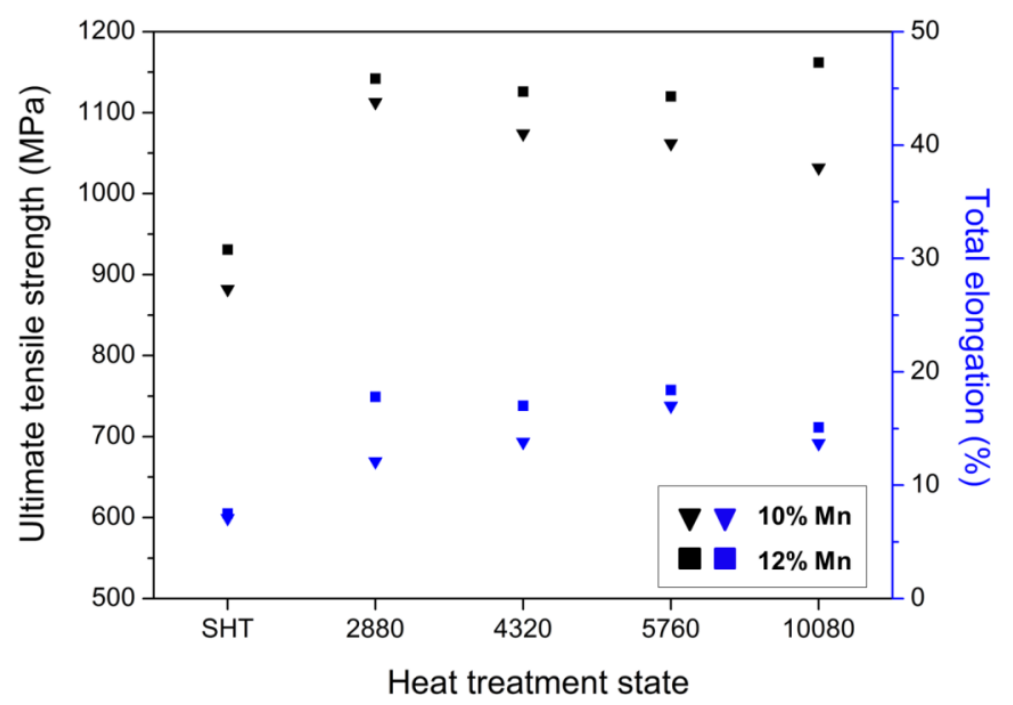

Figure 4-15 Comparison of UTS and TE between 10\% Mn and 12\% Mn alloys aged at $500{ }^{\circ} \mathrm{C}$ for different durations.

\subsection{Fractography analyses}

\subsubsection{The fracture surface after solution heat treatment}

Figure 4-16 displays the SEM fractographs of fracture surfaces in the SHT state. Figure 4-16(a) reveals a mixed fracture mode in solution-treated 7\% $\mathrm{Mn}$ alloy. Specifically, the flat facets exhibiting river patterns are typical of cleavage brittle fracture while cup-and-cone dimples are the characteristic feature of good, ductile fracture. Increasing Mn content led to the improvement of ductility. As shown in Figure 4-16(b), the proportion of dimple fracture in solution-treated $10 \% \mathrm{Mn}$ alloy is much higher than that in $7 \% \mathrm{Mn}$ alloy. $12 \% \mathrm{Mn}$ alloy exhibits a complete transgranular dimpled ductile fracture (Figure 4-16(c)). Compared to the uniform and shallow dimples in 7\% Mn and 10\% Mn alloys, the dimples in 12\% Mn alloy are relatively larger and deeper. Besides, bimodal dimples, i.e. both large and small dimples were observed in the $12 \%$ Mn alloy. 
Chapter 4 Brief study on microstructure and mechanical properties of Mn-based maraging steels
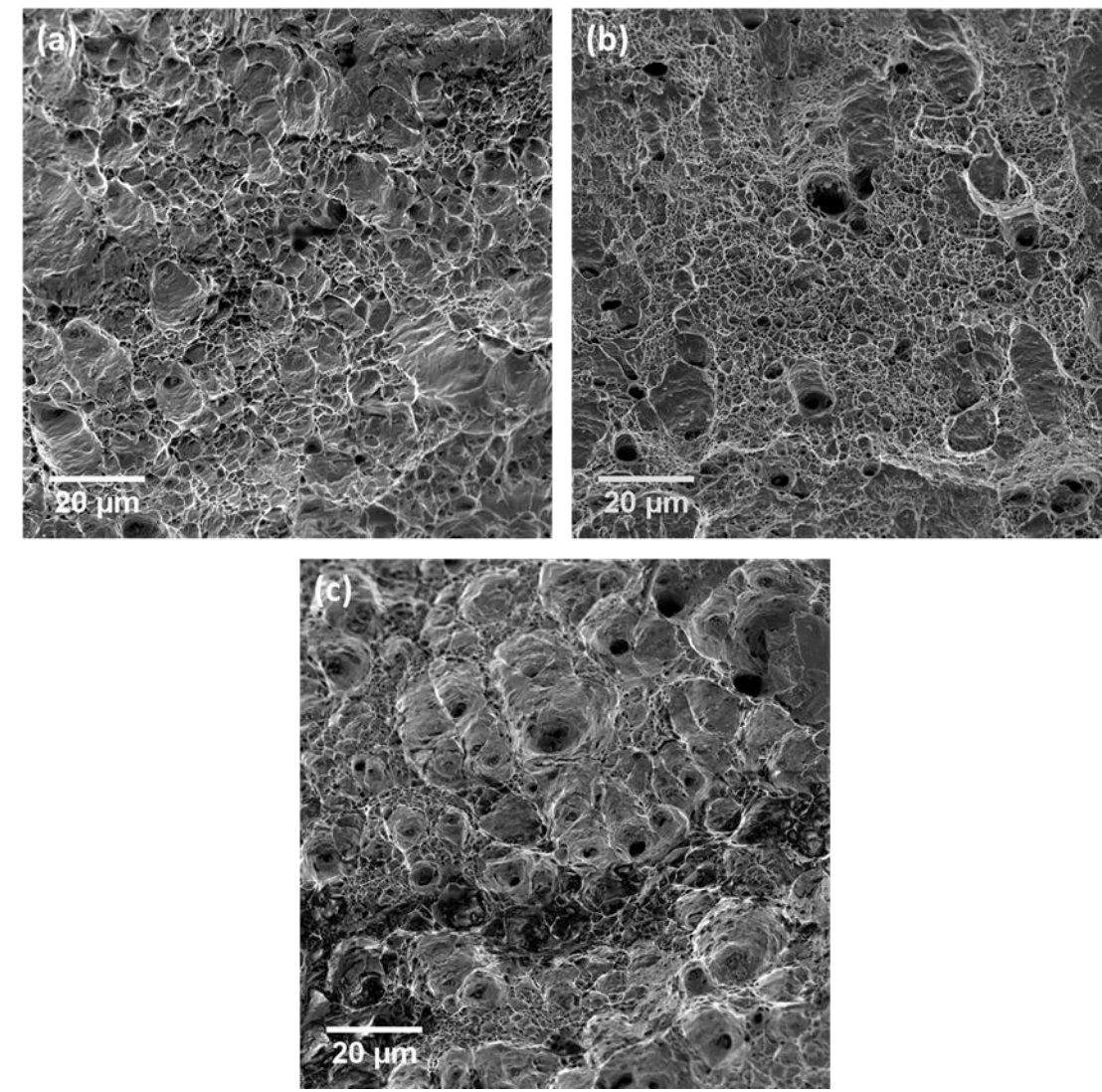

Figure 4-16 The fracture surface of solution-treated (a) $7 \% \mathrm{Mn}$; (b) $10 \% \mathrm{Mn}$ and (c) $12 \%$ Mn alloys.

\subsubsection{Intergranular brittleness in aged 7\% Mn alloy}

As the intergranular brittleness was most severe in 7\% $\mathrm{Mn}$ alloy, the 7\% $\mathrm{Mn}$ alloy was taken as example to study the effect of aging parameters on brittleness. Figure 4-17(a) reveals a nearly complete intergranular brittle fracture surface when aging at $420{ }^{\circ} \mathrm{C}$ for $10080 \mathrm{~min}$. Clear cracks along grain boundaries can be observed. Increasing aging temperature to $460{ }^{\circ} \mathrm{C}$ led to a small amount of cleavage fracture as shown in Figure 4-17(b). But the intergranular brittleness was still the dominant fracture mode. When subjected to the aging temperature of $500{ }^{\circ} \mathrm{C}$, the intergranular brittleness was still evident in the initial aging stage (Figure 4-17(c)), then the fracture mode gradually transformed into cleavage fracture as the aging time increases (Figure 4-17(d)). 

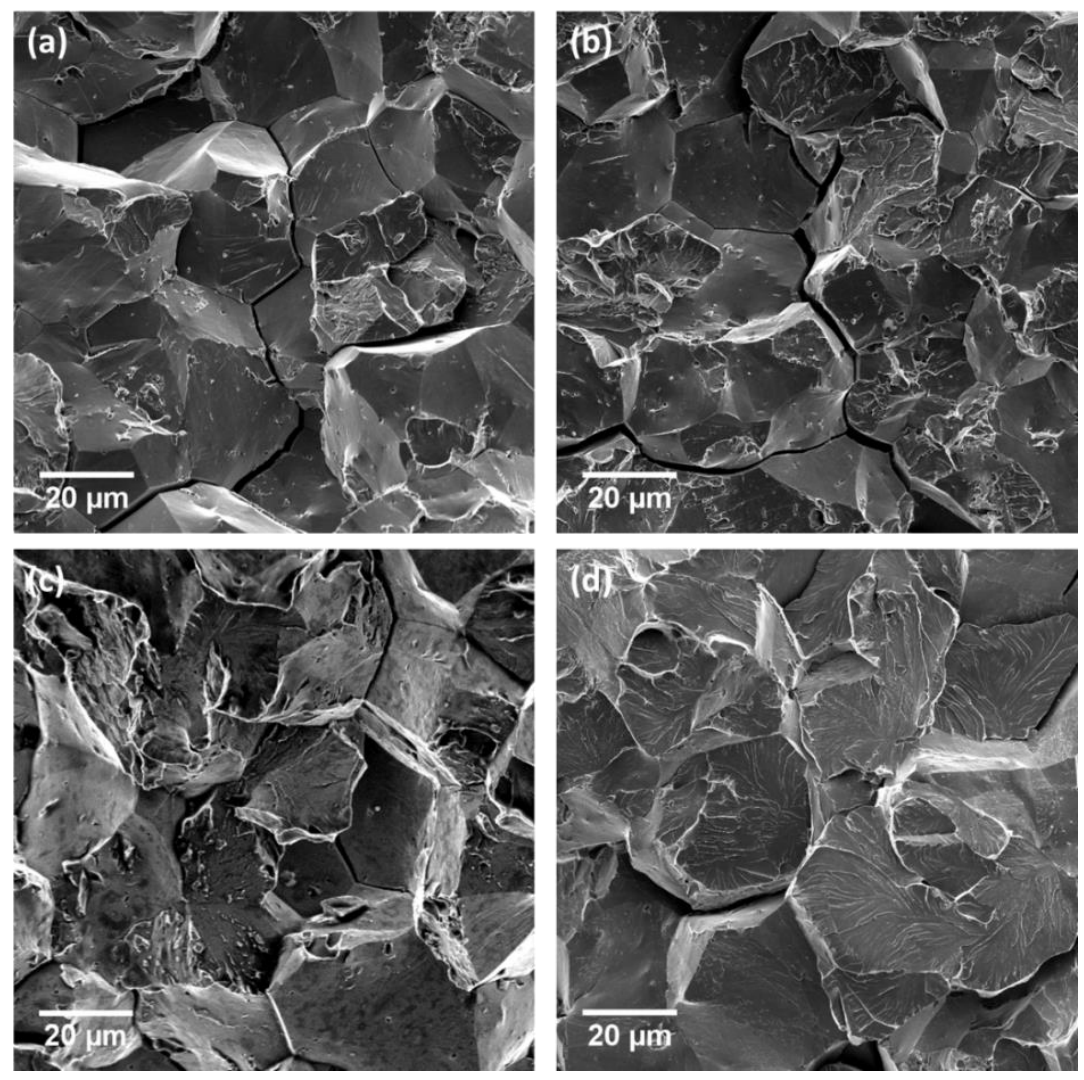

Figure 4-17 The fracture surface of $7 \% \mathrm{Mn}$ alloy aged at: (a) $420{ }^{\circ} \mathrm{C} / 10080 \mathrm{~min}$; (b) $460{ }^{\circ} \mathrm{C}$ / $10080 \mathrm{~min}$; (c) $500{ }^{\circ} \mathrm{C} / 60 \mathrm{~min}$ and (d) $500{ }^{\circ} \mathrm{C} / 10080 \mathrm{~min}$.

\subsubsection{Improved fracture behaviour in aged $12 \%$ Mn alloy}

As both the tensile result and microstructural observation indicated, $10 \%$ and $12 \%$ Mn alloys revealed similar mechanical properties and microstructure with a small variation in austenite fraction, the $12 \% \mathrm{Mn}$ alloy is representatively studied here to explore the effects of aging parameters on the fracture behaviour. At the aging temperature of $420{ }^{\circ} \mathrm{C}$, the $12 \% \mathrm{Mn}$ alloy revealed severe intergranular brittle fracture like 7\% Mn alloy shown in Figure 4-17(a). Increasing the aging temperature to $460{ }^{\circ} \mathrm{C}$ led to the improvement of fracture behaviour. The fracture mode transformed from cleavage fracture in the $460{ }^{\circ} \mathrm{C} / 4320 \mathrm{~min}$ state (Figure 4-18(a) and (b)) to quasi cleavage fracture mode in the $460{ }^{\circ} \mathrm{C} / 10080 \mathrm{~min}$ state (Figure 4-18(c) and (d)). The quasi cleavage is similar to the cleavage fracture but with an 
increased ductile proportion. As illustrated in Figure 4-18(c) and (d), small dimples seem to become dominant although intergranular fracture can still be observed.
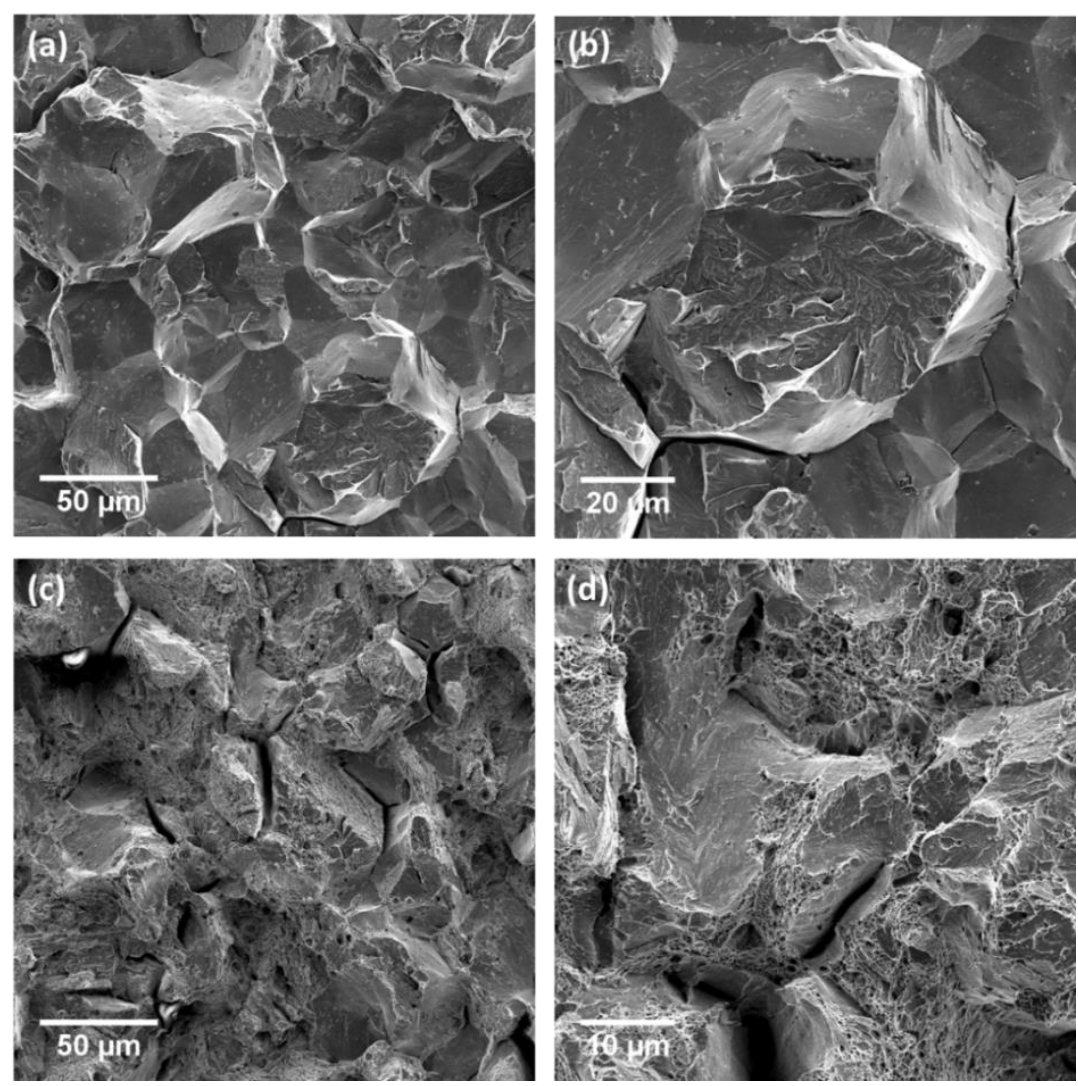

Figure 4-18 The fracture surface of $12 \% \mathrm{Mn}$ alloys aged at $460{ }^{\circ} \mathrm{C}$ for (a)(b) $4320 \mathrm{~min}$ and (c)(d) $10080 \mathrm{~min}$. The right column is the enlarged fractographs.

Although intergranular brittleness occurred at the onset of aging process $\left(500{ }^{\circ} \mathrm{C} / 10\right.$ min state, Figure 4-19(a)), further aging at $500{ }^{\circ} \mathrm{C}$ significantly improved the fracture performance of $12 \% \mathrm{Mn}$ alloy. After aging for $1440 \mathrm{~min}$, a microvoid coalescence fracture mode related to dimples was present with a small amount of river pattern (Figure 4-19(b)). In $500{ }^{\circ} \mathrm{C} / 10080$ min state, obvious necking and large reductions in area were clearly observed (not displayed here) and the fractograph is fully occupied by dimpled fracture mode (Figure 4-19(c)). It is found that the proportion of intergranular fracture increased immediately at the start of aging and with prolonged aging it gradually decreased. Besides, granular inclusions in the size range 100-200 $\mathrm{nm}$ were found at the bottom of some dimples as shown in Figure 4-19(d). It is worth noting that compared to the dimples in solution-treated 
stage (Figure 4-16), dimples were smoother and smaller in ductile aged samples (Figure 4-19(c) and (d)).
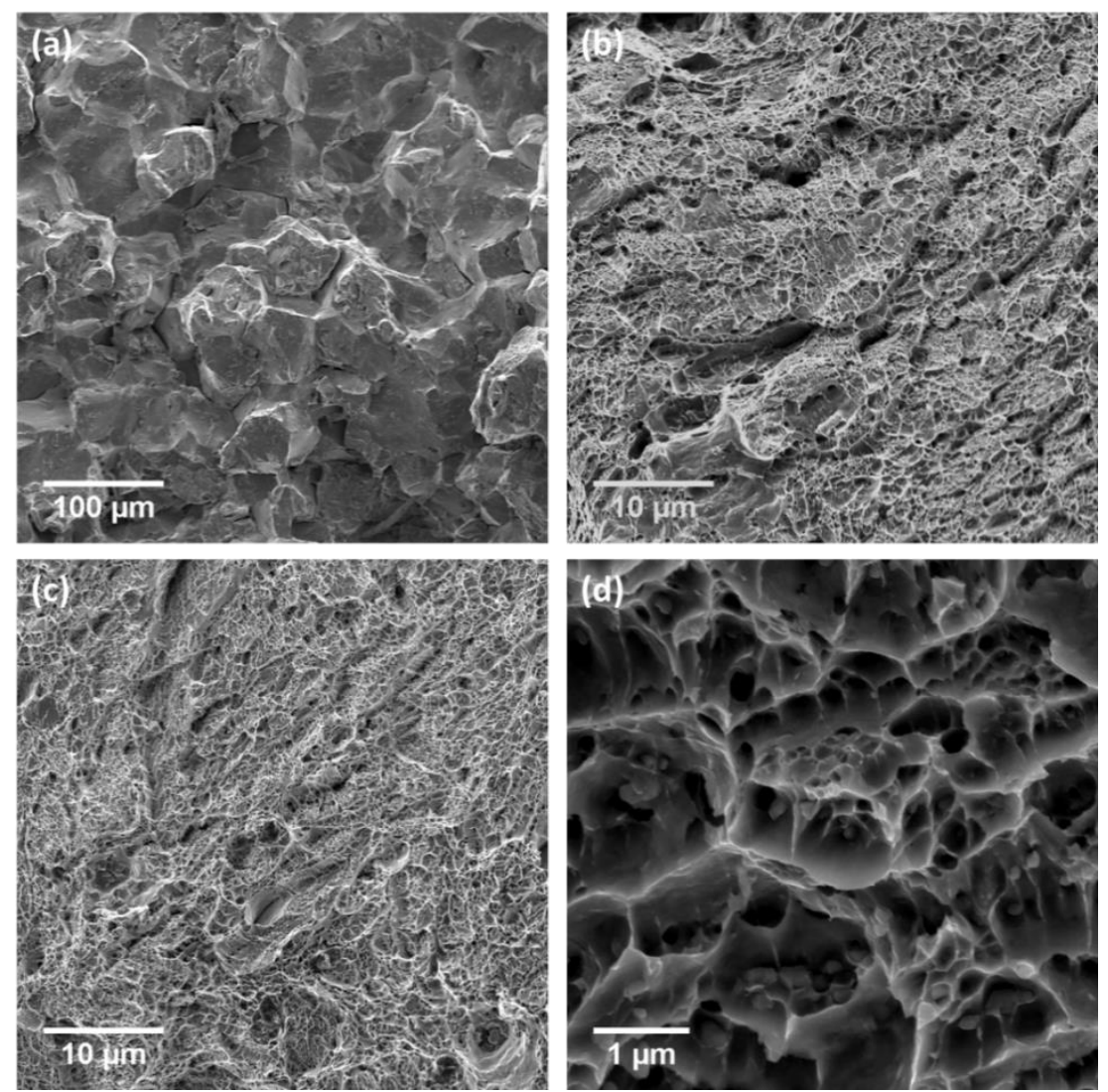

Figure 4-19 The fracture surface of $12 \% \mathrm{Mn}$ alloys aged at $500{ }^{\circ} \mathrm{C}$ for (a) $10 \mathrm{~min}$; (b) 1440 $\min$ and (c)(d) $10080 \mathrm{~min}$.

\subsection{Summary}

1. Thermodynamic calculation by MatCalc confirmed that the dominant equilibrium phases at aging temperature $420-500{ }^{\circ} \mathrm{C}$ are ferrite and austenite. The only precipitate phase predicted by MatCalc was Laves phase $\mathrm{Fe}_{3} \mathrm{Ti}(\mathrm{Mo})$. A trace of fcc phase $\mathrm{TiC}$ was found thermally stable over a wide temperature range up to $1300{ }^{\circ} \mathrm{C}$. The equilibrium $\gamma / \alpha$ transformation temperatures $\left(A_{e 3}\right)$ are calculated as: $7 \% \mathrm{Mn}$ alloy: $722{ }^{\circ} \mathrm{C} ; 10 \% \mathrm{Mn}$ alloy: $644{ }^{\circ} \mathrm{C} ; 12 \% \mathrm{Mn}$ alloy: $614^{\circ} \mathrm{C}$.

2. The addition of Mn has been demonstrated to refine both the prior austenite grains and martensite packets via reducing $A_{e 3}$ and $M_{s}$ temperatures. This 
grain refinement is partially responsible for the higher strength and ductility in $12 \% \mathrm{Mn}$ alloy in the SHT state.

3. Phase identification by XRD confirmed the formation of reverted austenite during aging. Besides, XRD analyses revealed the existence of $\varepsilon$-martensite in over-aged samples, but no evidence of precipitates was found in XRD patterns.

4. Aging at $460{ }^{\circ} \mathrm{C}$ or $500{ }^{\circ} \mathrm{C}$ exhibited a typical hardness evolution of precipitation-strengthened alloys. Higher aging temperature led to a more rapid hardening towards peak hardness followed by a moderate decrease in hardness in the overaged region. A slower hardening kinetics but more significant hardening effect existed at $420{ }^{\circ} \mathrm{C}$. In terms of the Mn content of alloy, a quicker response to thermal treatment and more significant hardening effect were observed in $12 \% \mathrm{Mn}$ alloy when compared to other two alloys.

5. Embrittlement occurred in the samples aged at lower temperatures or at higher temperatures but for short times. Increasing aging temperatures and durations could significantly improve the embrittlement phenomenon. Higher Mn content was also demonstrated to benefit the ductility as well. The $12 \%$ Mn alloy aged at $500{ }^{\circ} \mathrm{C}$ for 5760 min exhibited a UTS of $1120 \mathrm{MPa}$ with TE of $18.4 \%$.

6. The fracture mode of solution-treated samples is a mix of cleavage brittle fracture and dimples fracture. Higher Mn content corresponded to an increase of the proportion of dimples fracture. Intergranular brittle fracture was observed in the early aging stage. The embrittlement phenomena were much improved after prolonged aging process and the fracture mode gradually transformed to a transgranular dimpled ductile fracture. Both higher $\mathrm{Mn}$ content and higher aging temperature were found to accelerate the improvement of ductility. 


\section{Chapter 5 Characterization of L2 1 -ordered $\mathrm{Ni}_{2} \mathrm{TiAl}$ intermetallic phase and its precipitation behaviour}

\subsection{Introduction}

The previous hardness study in Section 4.4 revealed a rapid and significant hardening during aging in the three Mn-based maraging steels and evidence has suggested that the hardening is mainly attributed to the formation of an unknown type of precipitate. Therefore, the primary objective addressed in this study is to investigate the nature of the precipitate. In this chapter, the chemical composition, coarsening behaviour, crystal structure and coherency of precipitates will be studied aiming to understand their effects on the strengthening behaviour of Mn-based maraging steels respectively.

\subsection{Experimental details}

The precipitates formed in 7\%, 10\% and 12\% Mn alloys under different aging treatments were investigated. The solution-treated samples were aged at $420{ }^{\circ} \mathrm{C}$, $460{ }^{\circ} \mathrm{C}$ and $500{ }^{\circ} \mathrm{C}$ for $10,30,60,120,240,480,1440,2880,4320,5760$ and 10080 mins. Various TEM techniques including bright field, electron diffraction, dark field and EDS were employed to obtain the information about size, composition and the crystal structure of precipitates. Most of these investigations were carried out on overaged samples as the ultrafine precipitates formed prior to the peak strengthening were difficult to be identified under conventional TEM observation. In this case, carbon extracted replicas were used for the investigation of early precipitation. Complementary studies on the crystal structure and core/shell structure were performed on HREM and HAADF. 


\subsection{Microstructural evolution}

\subsubsection{The effect of Mn content on the precipitation}

Figure 5-1 presents the bright-field TEM micrographs of precipitates in 7\% $\mathrm{Mn}$ and $12 \% \mathrm{Mn}$ alloys aged at $500{ }^{\circ} \mathrm{C}$. The microstructural evolution of precipitates in the $7 \%$ Mn alloy aged at $500{ }^{\circ} \mathrm{C}$ is illustrated in the left column. The $480 \mathrm{~min}$ aged sample (Figure 5-1(a)), corresponding to the peak aging hardening, exhibited a fine, highly dispersed distribution of precipitates with weak contrast. Further aging led to the increase of the size of precipitates, as shown in the $2880 \mathrm{~min}$ aged sample (Figure 5-1(c)). Precipitate coarsening, which resulted in a significant decrease of the number density, was obvious in the 10080 min aged sample (Figure 5-1(e)). For the $12 \% \mathrm{Mn}$ alloy, the precipitates in the $480 \mathrm{~min}$ aged sample have a larger average size and higher number density compared to the precipitates in the $7 \% \mathrm{Mn}$ alloy for the same aging condition. Analogous to the $7 \% \mathrm{Mn}$ alloy, further aging to $2880 \mathrm{~min}$ and then $10080 \mathrm{~min}$ resulted in a significant increase in the precipitate size but a decrease in the number density (Figure 5-1(d) and (f)). 
Chapter 5 Characterization of $\mathrm{L2}_{1}$-ordered $\mathrm{Ni}_{2} \mathrm{TiAl}$ intermetallic phase and its precipitation behaviour
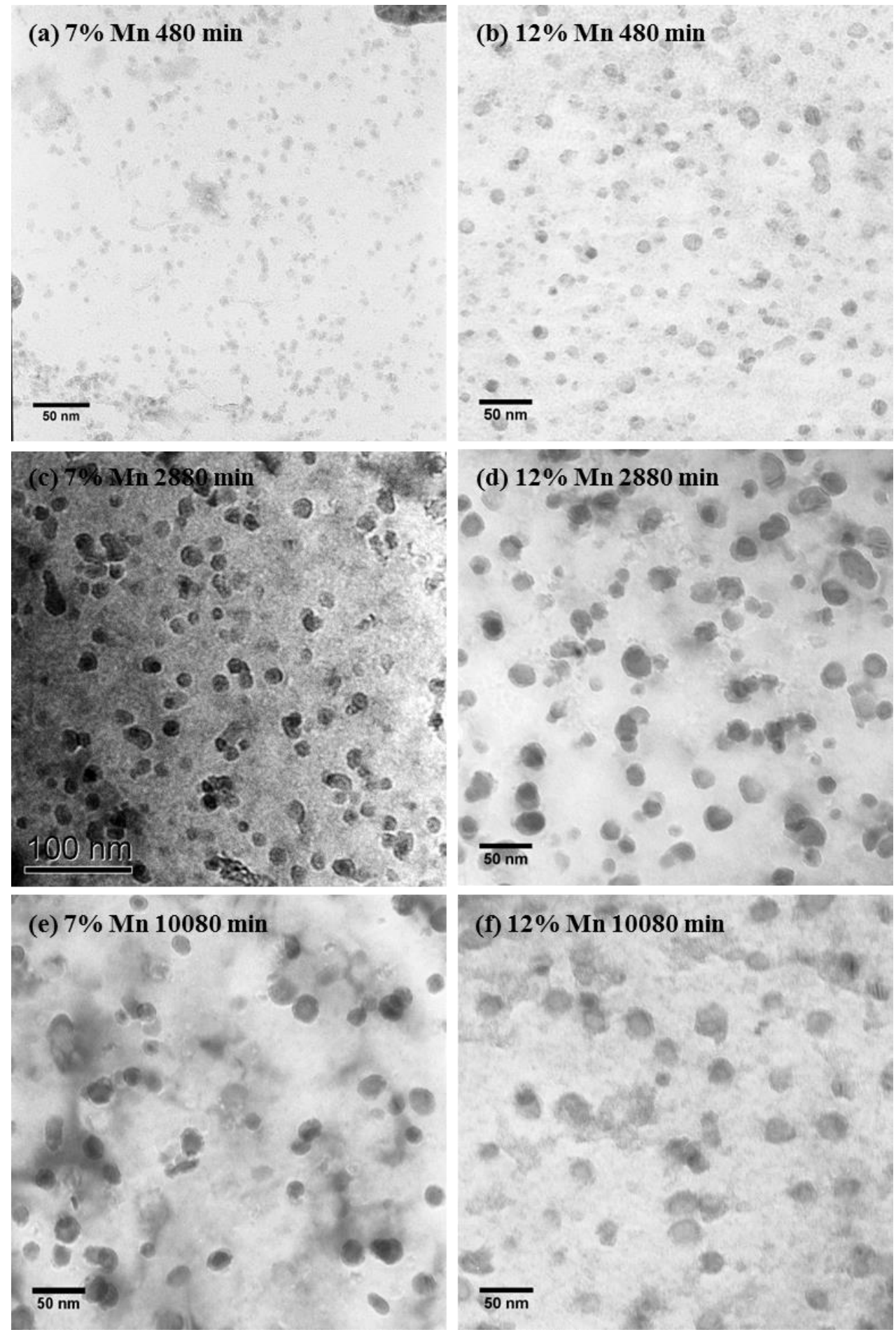

Figure 5-1 Bright-field TEM micrographs of precipitates formed at $500{ }^{\circ} \mathrm{C}$. Left: $7 \% \mathrm{Mn}$ alloy for (a) $480 \mathrm{~min}$ (c) $2880 \mathrm{~min}$ and (e) $10080 \mathrm{~min}$; right: 12\% Mn alloy for (b) $480 \mathrm{~min}$ (d) $2880 \mathrm{~min}$ and (f) $10080 \mathrm{~min}$. 


\subsubsection{The effect of aging temperature on precipitation}

The aging temperature is one of the key factors to determine the population and size of precipitates. It is believed that lower aging temperatures can introduce a high number density of fine precipitates, and thus a better strengthening contribution. On the other hand, the kinetics of precipitation is, to a large extent, also associated with the aging temperature. A relatively high temperature is generally desired by steelmakers to reduce the cycle time of aging process. Therefore, investigation is needed to determine the optimum aging temperature meeting both the thermodynamics and kinetics requirement.

The representative $12 \% \mathrm{Mn}$ alloy subjected to different aging temperatures was studied. As shown in Figure 5-2(a), the precipitates experienced an extremely slow growth process when aging at $420{ }^{\circ} \mathrm{C}$. A small average size of $\sim 5.5 \mathrm{~nm}$ was achieved after aging for $10080 \mathrm{~min}$. A more rapid growth was observed at $460{ }^{\circ} \mathrm{C}$ where the average size grew up to $\sim 7.5 \mathrm{~nm}$ after aging for $10080 \mathrm{~min}$ (Figure 5-2(b)), and a moderate increase in the number density is also visible. Further increasing the aging temperature to $500{ }^{\circ} \mathrm{C}$ led to a significant increase in the size but a decrease in the number density: the precipitates in the $10080 \mathrm{~min}$ aged sample have an average size of $19.4 \pm 6.4 \mathrm{~nm}$ (Figure 5-2(c)). 
Chapter 5 Characterization of $\mathrm{L}_{1}$-ordered $\mathrm{Ni}_{2} \mathrm{TiAl}$ intermetallic phase and its precipitation behaviour

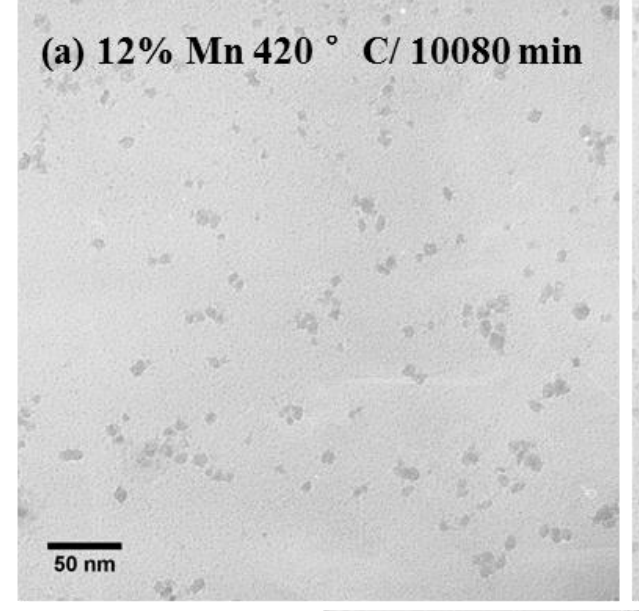

(b) $12 \% \mathrm{Mn} 460 \mathrm{C} / 10080 \mathrm{~min}$

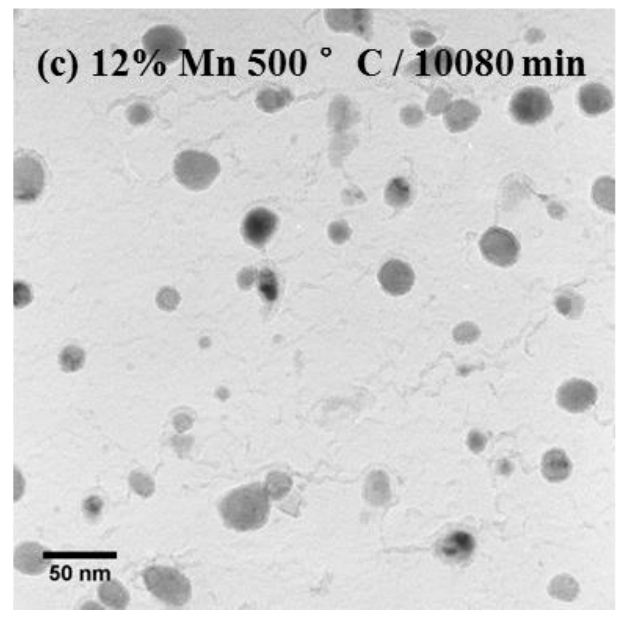

Figure 5-2 TEM micrographs of precipitates on carbon extraction replicas of $12 \% \mathrm{Mn}$ alloy aged at (a) $420{ }^{\circ} \mathrm{C}$, (b) $460{ }^{\circ} \mathrm{C}$ and (c) $500{ }^{\circ} \mathrm{C}$ for $10080 \mathrm{~min}$.

\subsection{Coarsening of precipitates}

\subsubsection{Size distribution of precipitates}

Based on the TEM observation of precipitates, the precipitate size distributions (PSDs) were analysed and the representative results are displayed in Figure 5-3. Due to the limitation of the resolution of extraction replicas under TEM observation, precipitates with an equivalent radius below $1 \mathrm{~nm}$ could not be counted. The PSD function (g) was plotted as a function of reduced precipitate radius $(\rho=r / \bar{r})$ to allow comparison with the LSW model expressed as [76]: 


$$
\int_{0}^{3 / 2} \rho^{2} h(\rho) d \rho=\frac{9}{4}
$$

where $\rho^{2} h(\rho)$ is the theoretical distribution function. As displayed in Figure 5-3, the experimental PSDs results roughly agree with the theoretical PSD function superimposed on the histograms and the agreement in the early aging stage is better than that after long-term aging. Despite the variation of the average precipitate size, the PSDs of the three alloys exhibit a similar shape for the same aging condition, which means that the effect of Mn content was not that significant on the precipitate size distributions. In terms of the aging time, the PSDs of the three alloys were all narrow in the beginning $\left(500{ }^{\circ} \mathrm{C} / 480 \mathrm{~min}\right)$ and fitted well with the PSD function predicted by LSW theory (Figure 5-3(a), (d) and (g)), but after aging for $2880 \mathrm{~min}$ the PSDs yielded a broader shape than the theoretical PSD distribution (Figure 5-3(b), (e) and (h)), and this discrepancy maintained at this level to the end of aging process (10080 min state), as shown in Figure 5-3(c), (f)and (i). 


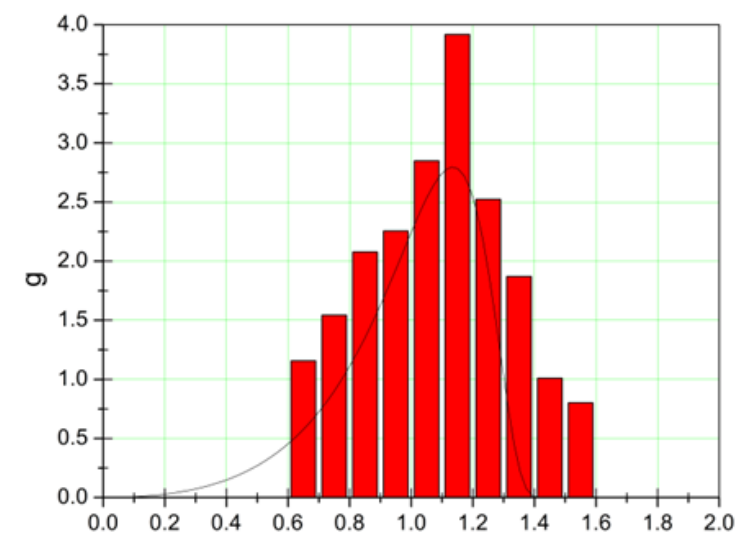

(a) $7 \% \mathrm{Mn}, 500^{\circ} \mathrm{C} / 480 \mathrm{~min}$

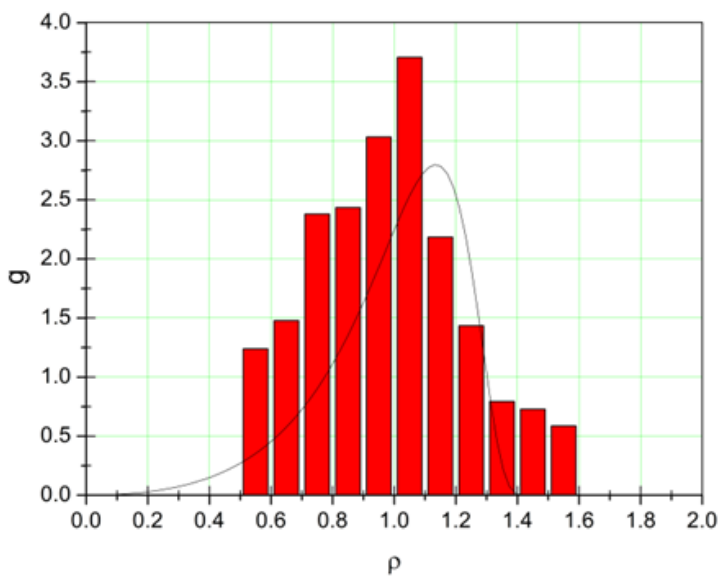

(d) $10 \% \mathrm{Mn}, 500^{\circ} \mathrm{C} / 480 \mathrm{~min}$

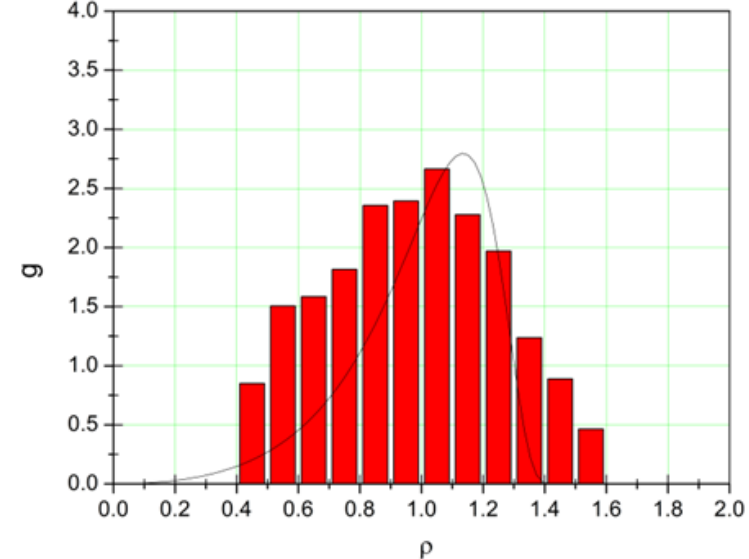

(b) $7 \% \mathrm{Mn}, 500^{\circ} \mathrm{C} / 2880 \mathrm{~min}$

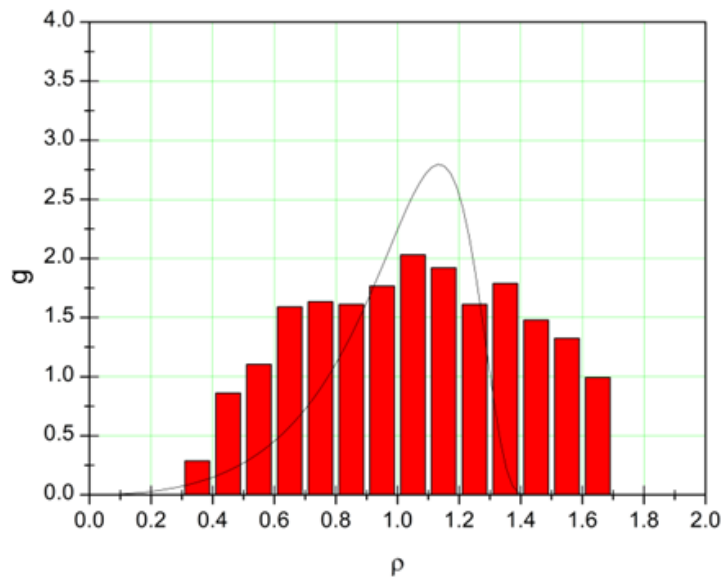

(e) $10 \% \mathrm{Mn}, 500^{\circ} \mathrm{C} / 2880 \mathrm{~min}$

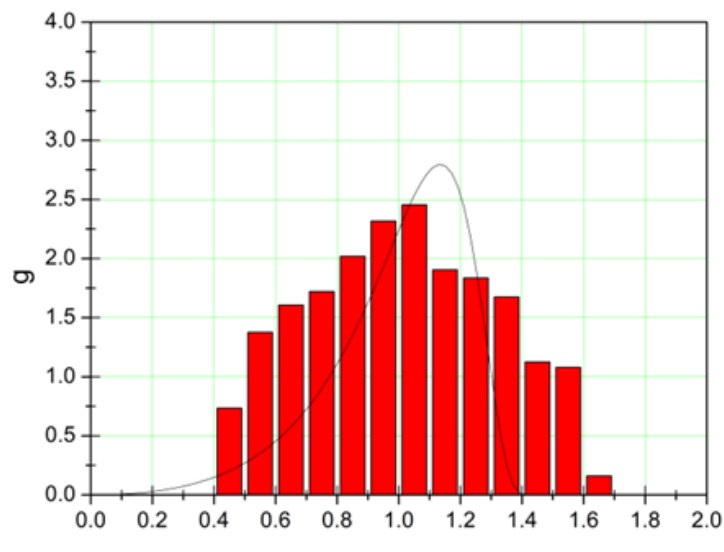

(c) $7 \% \mathrm{Mn}, 500^{\circ} \mathrm{C} / 10080 \mathrm{~min}$

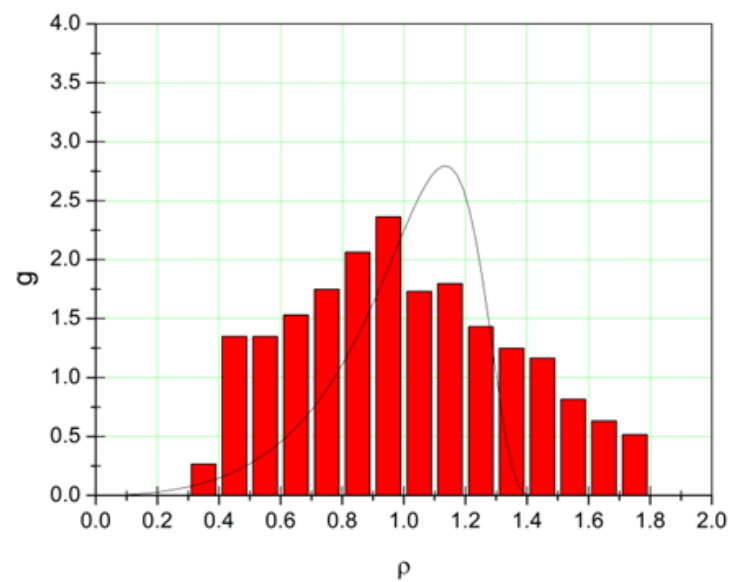

(f) $10 \% \mathrm{Mn}, 500^{\circ} \mathrm{C} / 10080 \mathrm{~min}$ 


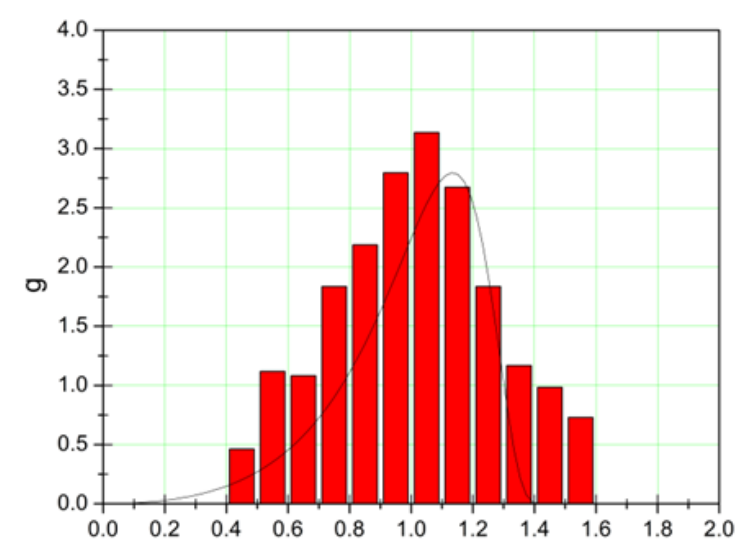

$\rho$

(g) $12 \% \mathrm{Mn}, 500^{\circ} \mathrm{C} / 480 \mathrm{~min}$

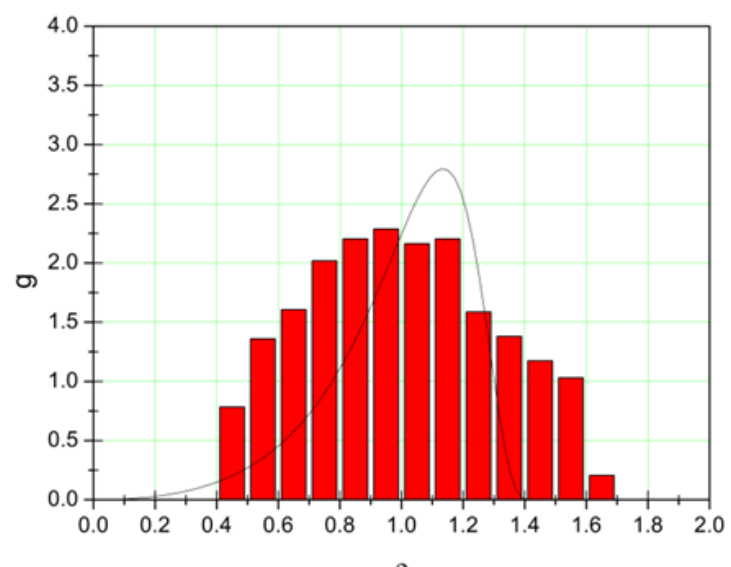

(h) $12 \% \mathrm{Mn}, 500^{\circ} \mathrm{C} / 2880 \mathrm{~min}$

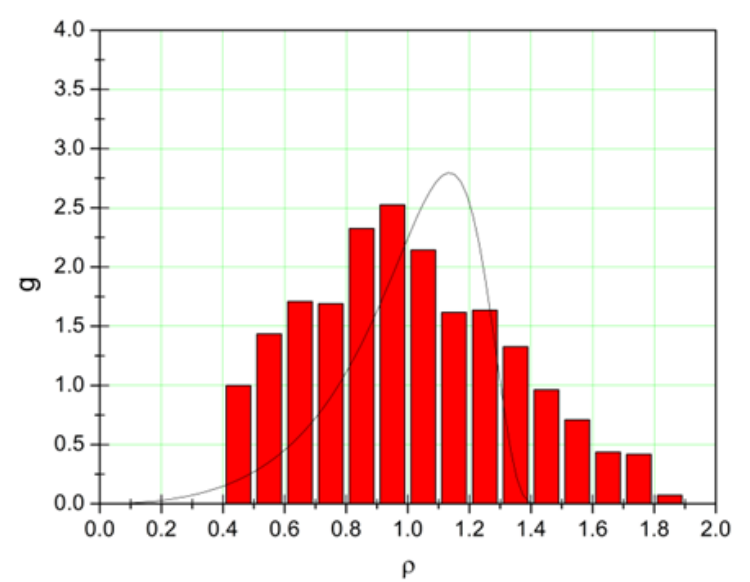

(i) $12 \% \mathrm{Mn}, 500^{\circ} \mathrm{C} / 10080 \mathrm{~min}$

Figure 5-3 Examples of PSDs plotted for various aged samples: $7 \% \mathrm{Mn}$ alloy (a) $500{ }^{\circ} \mathrm{C} / 480 \mathrm{~min}$, (b) $500{ }^{\circ} \mathrm{C} / 2880 \mathrm{~min}$ and (c) $500^{\circ} \mathrm{C} / 10080 \mathrm{~min}$; $10 \% \mathrm{Mn}$ alloy (d) $500{ }^{\circ} \mathrm{C} / 480 \mathrm{~min}$, (e) $500{ }^{\circ} \mathrm{C} / 2880 \mathrm{~min}$ and (f) $500{ }^{\circ} \mathrm{C} / 10080 \mathrm{~min} ; 12 \% \mathrm{Mn}$ alloy (g) $500{ }^{\circ} \mathrm{C} / 480 \mathrm{~min}$, (h) $500{ }^{\circ} \mathrm{C} / 2880 \mathrm{~min}$ and

(i) $500^{\circ} \mathrm{C} / 10080 \mathrm{~min}$. The histograms of the PSD function, $\mathrm{g}$, are plotted as a function of reduced precipitate radius $(\rho=r / \bar{r})$. Theoretical LSW distribution is presented as well for comparison. 
Chapter 5 Characterization of $\mathrm{L}_{1}$-ordered $\mathrm{Ni}_{2} \mathrm{TiAl}$ intermetallic phase and its precipitation behaviour

\subsubsection{The growth and coarsening kinetics of precipitate}

The growth kinetics at the early stage of precipitation was studied. It is found that the size of early precipitates followed a linear increase with aging time, after which the rate of increase appeared to change indicating the initiation of the coarsening process (Figure 5-4). The trend of curves is consistent with the description of precipitate size-time relationship in Figure 2-5. Besides, the growth rate of precipitates in $7 \%$ Mn alloy is found slower than that in $12 \%$ Mn alloy.
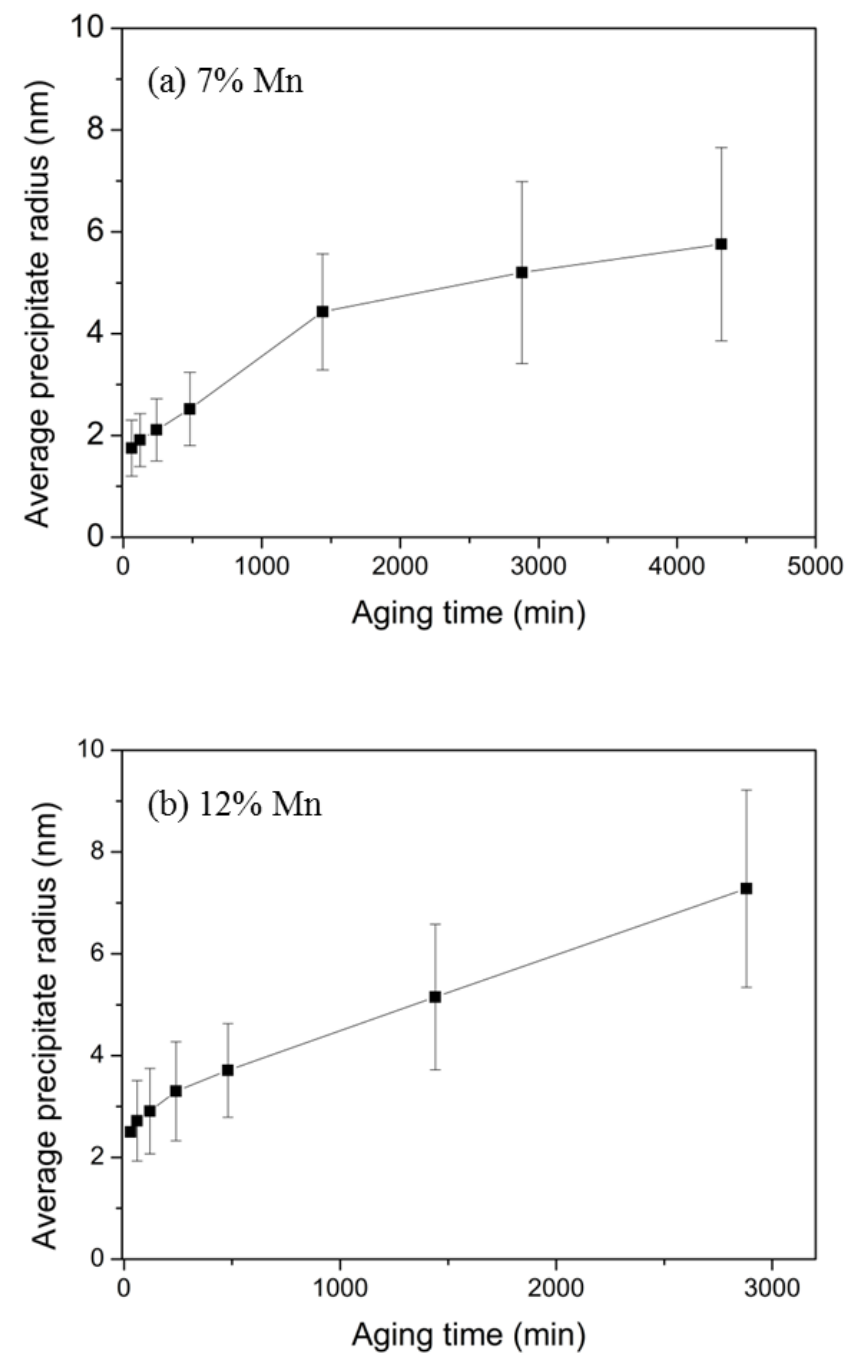

Figure 5-4 Evolution of the mean precipitate size $\overline{\mathrm{r}}$ as a function of aging time at $500{ }^{\circ} \mathrm{C}$ in (a) $7 \% \mathrm{Mn}$ alloy and (b) $12 \% \mathrm{Mn}$ alloy. 
The precipitate coarsening in the $7 \%, 10 \%$ and $12 \%$ Mn alloys aged at $500{ }^{\circ} \mathrm{C}$ was assessed by plotting mean precipitate radius $\bar{r}^{3}$ (Table 5-1) as a function of aging time $t$ (Figure 5-5). Except the point of $500{ }^{\circ} \mathrm{C} / 10080 \mathrm{~min}$ state in $12 \% \mathrm{Mn}$ alloy, the linear slopes in Figure 5-5 confirm to the relationship between the mean precipitate radius and aging time:

$$
\bar{r}^{3}-r_{c 5}^{3}=K_{R} t
$$

This indicates that the coarsening kinetics of precipitates in the three alloys follows the diffusion-controlled coarsening kinetics predicted by LSW theory. The slopes in Figure 5-5 reveal that the coarsening rate constants increase with the Mn contents of alloy. Besides, the $\bar{r}^{3}$ of $12 \% \mathrm{Mn}$ alloy aged at $500{ }^{\circ} \mathrm{C} / 10080 \mathrm{~min}$, which shows a deviation from the expected value by LSW model. This could indicate a change in coarsening mechanism or possibly a change in precipitate structure. Further work would be required to understand this change.

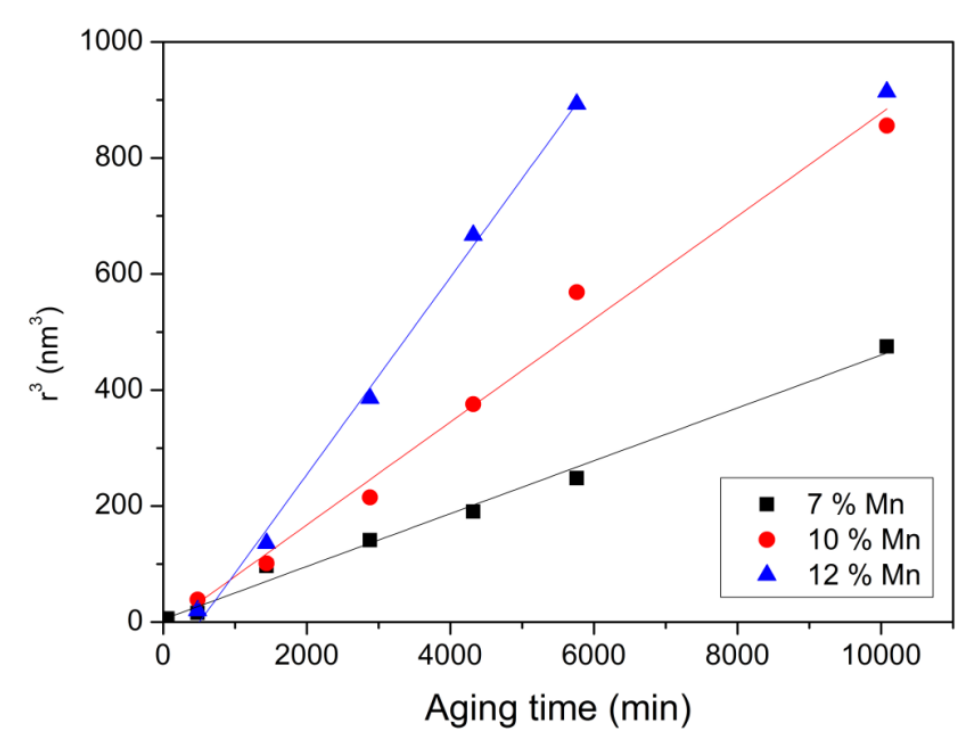

Figure 5-5 Evolution of the mean precipitate radius $\bar{r}^{3}$ as a function of aging time $t$, in 7\% $\mathrm{Mn}, 10 \% \mathrm{Mn}$ and $12 \% \mathrm{Mn}$ alloys aged at $500{ }^{\circ} \mathrm{C}$. 
Table 5-1 Experimental mean size of precipitates $(\bar{r})$ and coarsening rate constant $\left(K_{R}\right)$ of different alloys aged at $500{ }^{\circ} \mathrm{C}$.

\begin{tabular}{|c|c|c|c|c|c|c|}
\hline \multirow{2}{*}{$\begin{array}{l}\text { Aging time } \\
(\min )\end{array}$} & \multicolumn{2}{|c|}{$7 \% \mathrm{Mn}$} & \multicolumn{2}{|c|}{$10 \% \mathrm{Mn}$} & \multicolumn{2}{|c|}{$12 \% \mathrm{Mn}$} \\
\hline & $\bar{r}(\mathrm{~nm})$ & $K_{R}\left(\mathrm{~m}^{3} \mathrm{~s}^{-1}\right)$ & $\bar{r}(\mathrm{~nm})$ & $K_{R}\left(\mathrm{~m}^{3} \mathrm{~s}^{-1}\right)$ & $\bar{r}(\mathrm{~nm})$ & $K_{R}\left(\mathrm{~m}^{3} \mathrm{~s}^{-1}\right)$ \\
\hline 480 & $2.52 \pm 0.72$ & - & $3.39 \pm 1.28$ & - & $3.71 \pm 1.43$ & - \\
\hline 1440 & $4.43 \pm 1.14$ & - & $4.66 \pm 1.56$ & - & $5.15 \pm 1.94$ & - \\
\hline 2880 & $5.2 \pm 1.79$ & - & $5.99 \pm 2.41$ & - & $7.28 \pm 2.21$ & - \\
\hline 4320 & $5.76 \pm 1.9$ & - & $7.22 \pm 2.44$ & - & $8.74 \pm 3.15$ & - \\
\hline 5760 & $6.29 \pm 1.8$ & - & $8.29 \pm 2.78$ & - & $9.63 \pm 3.17$ & $2.83 \times 10^{-30}$ \\
\hline 10080 & $7.81 \pm 2.38$ & $0.76 \times 10^{-30}$ & $9.5 \pm 3.33$ & $1.48 \times 10^{-30}$ & $9.71 \pm 3.19$ & - \\
\hline
\end{tabular}




\subsection{Chemical composition of precipitates}

The chemical composition of precipitates in Mn-based maraging steels is under debate in the literature [18][44][135][217][218]. Apart from the commonly reported $\theta$-NiMn precipitates in Fe- Mn-Ni maraging steels, Heo et al. [217] found a type of precipitate with the chemical composition agreeing with $\mathrm{Ni}_{2} \mathrm{MnAl}$ phase in an $\mathrm{Fe}$ 8.3Mn-8.2Ni-4.2Al alloy. The investigation of the newly developed 9-12\% $\mathrm{Mn}$ maraging steels with minor additions of $\mathrm{Ni}, \mathrm{Ti}, \mathrm{Al}$ and $\mathrm{Mo}$, which are chemically similar to the materials in our study, reported the precipitates were enriched in $\mathrm{Ni}$, $\mathrm{Ti}$ and $\mathrm{Al}[18][44]$. It was initially speculated the precipitates might be $\gamma^{\prime}-\mathrm{Ni}_{3}(\mathrm{Ti}, \mathrm{Al})$ phase which is the most common precipitates in conventional $18 \mathrm{Ni}$ maraging steels. Later, APT analysis, revealed that the average chemical composition was close to $\mathrm{Ni}_{50}(\mathrm{Al}, \mathrm{Ti}, \mathrm{Mn})_{50}$ [135]. However, a more recent study proposed the formation of $\mathrm{NiMn}$ or/and $\mathrm{Ni}_{2} \mathrm{MnAl}$ precipitates depending on the $\mathrm{Al}$ contents in these $\mathrm{Mn}$ maraging steels [219]. Moreover, in a ternary Fe-10Mn-1Pd biodegradable maraging steel, the addition of $\mathrm{Pd}$ was found to promote the formation of ( $\mathrm{Pd}, \mathrm{Mn})$-rich precipitates. All the evidence suggests that the chemical compositions of precipitates in Mn-based maraging steels are variable and, to a large extent, depend on the elemental concentrations of alloys.

TEM-EDS analyses were conducted on thin foil samples and the representative spectra of both precipitates and matrix $\left(7 \% \mathrm{Mn}, 500{ }^{\circ} \mathrm{C} / 12960 \mathrm{~min}\right)$ are presented in Figure 5-6. The spectrum in Figure 5-6(b) indicates that the precipitate contained $\mathrm{Ni}$, Ti and Al when compared to the surrounding matrix (Figure 5-6(c)). The major elements of the matrix, Fe and Mn, were also detected by the EDS analysis of precipitates (Figure 5-6(b)). Some researchers suggested that the $\mathrm{Fe}$ and $\mathrm{Mn}$ concentrations were due to the residual matrix above or below precipitates [85], but this viewpoint is doubtable as the $\mathrm{Mn} / \mathrm{Fe}$ mass ratio in Figure 5-6(b) is much higher than that in Figure 5-6(c). 
Chapter 5 Characterization of $\mathrm{L}_{1}$-ordered $\mathrm{Ni}_{2} \mathrm{TiAl}$ intermetallic phase and its precipitation behaviour

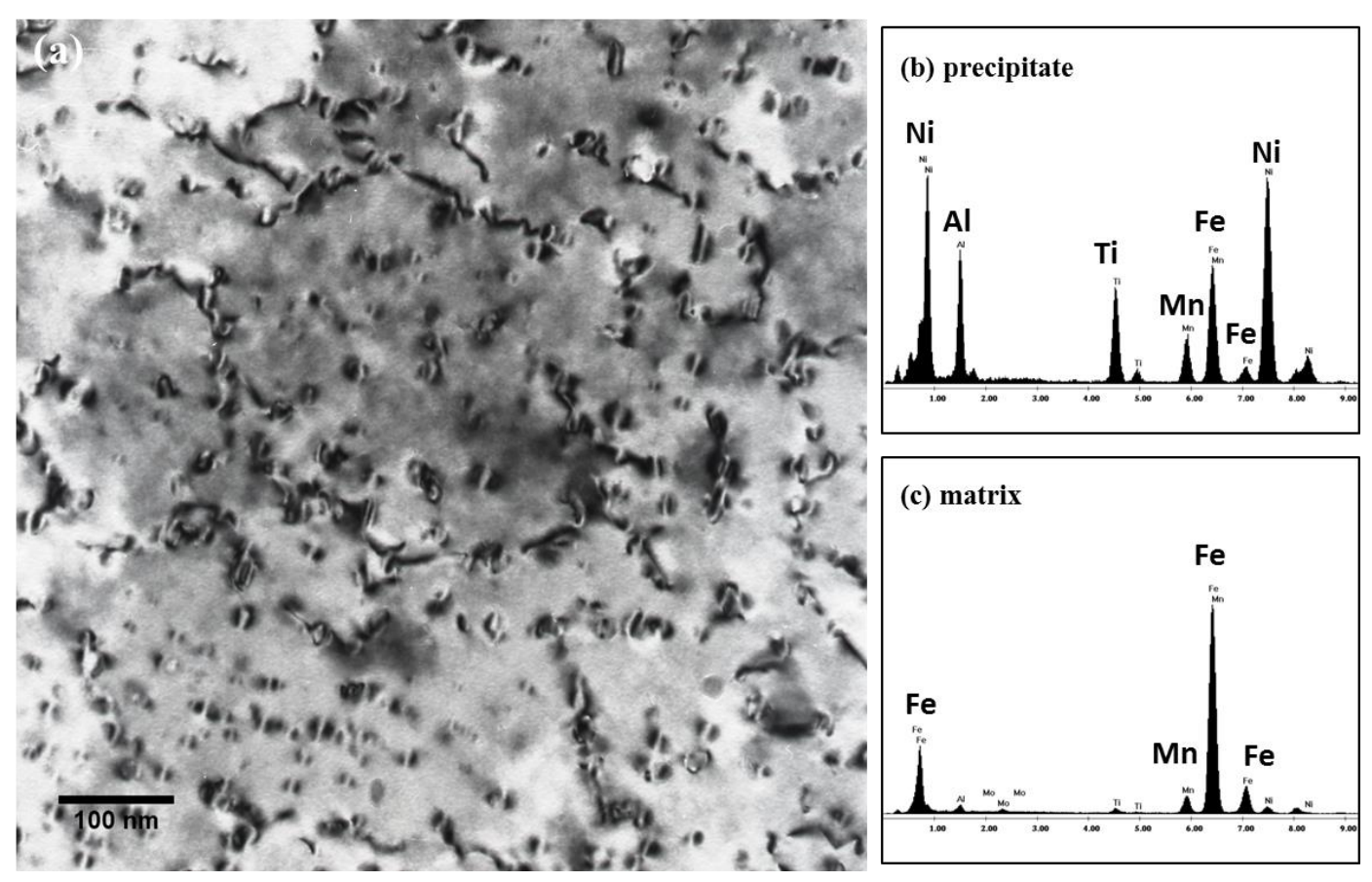

Figure 5-6 (a) Bright-field TEM image of 7\% Mn alloy aged at $500{ }^{\circ} \mathrm{C}$ for $12960 \mathrm{~min}$ and TEM-EDS spectra of (b) precipitate and (c) matrix.

In order to minimize the interference from the matrix, carbon extraction replicas were utilized for further compositional analyses. Representative STEM-EDS analysis was conducted on $12 \% \mathrm{Mn}$ alloy aged at $500{ }^{\circ} \mathrm{C}$ for $2880 \mathrm{~min}$ along the horizontal line crossing a precipitate, as shown in Figure 5-7. The corresponding concentration profiles of $\mathrm{Al}, \mathrm{Ti}, \mathrm{Mn}, \mathrm{Fe}$ and $\mathrm{Ni}$ are plotted below the STEM micrograph. 
Chapter 5 Characterization of $\mathrm{L}_{1}$-ordered $\mathrm{Ni}_{2} \mathrm{TiAl}$ intermetallic phase and its precipitation behaviour

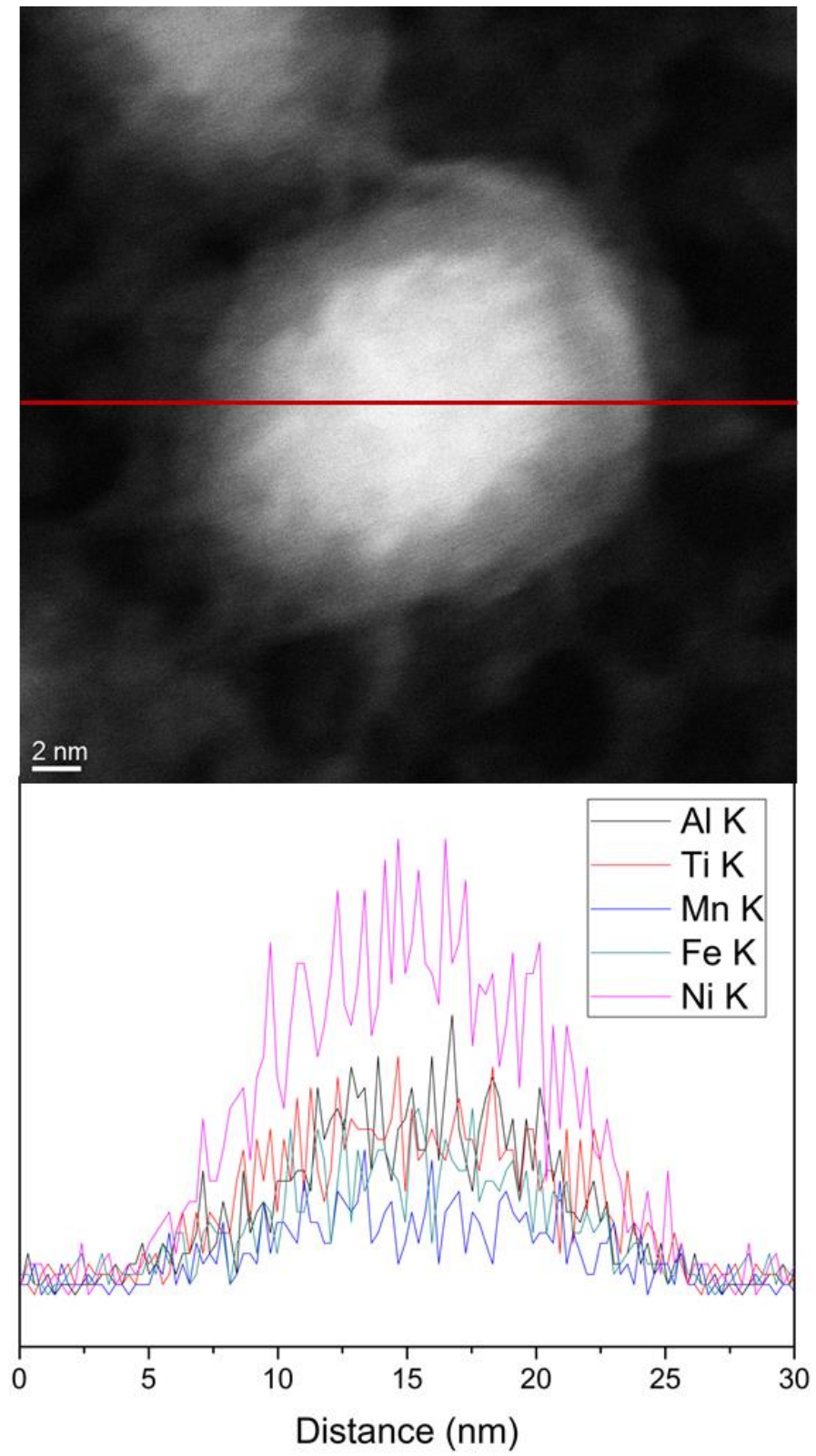

Figure 5-7 STEM micrograph of precipitates in $12 \% \mathrm{Mn}$ alloy aged at $500{ }^{\circ} \mathrm{C}$ for $2880 \mathrm{~min}$ and EDS results of concentration profiles measured along the red line.

As displayed in Figure 5-7, the STEM-EDS analysis on replica sample confirms that the precipitates mainly comprised $\mathrm{Ni}$, Ti and $\mathrm{Al}$ with some segregation of $\mathrm{Fe}$ and $\mathrm{Mn}$. The considerable $\mathrm{Fe}$ and $\mathrm{Mn}$ concentration detected demonstrates that the segregation of Fe and $\mathrm{Mn}$ in precipitates is not an artefact. The APT study conducted by Millán et al. [135] also confirmed the existence of $\mathrm{Fe}$ and $\mathrm{Mn}$ atoms in precipitates and 20 at.\% Fe and 25 at.\% Mn were detected by APT in the precipitates 
in a $9 \% \mathrm{Mn}$ alloy aged at $450{ }^{\circ} \mathrm{C}$ for $192 \mathrm{~h}$. As mentioned in Section 2.2.3.1, similar phenomenon of matrix elements segregating to precipitates has been reported in several NiAl-strengthened steels [32][40][60][78][81]. It is worth noting that in an $\mathrm{Fe}-\mathrm{Ni}$-Al-Ti-Cr alloy, two independent precipitates, $\mathrm{NiAl}$ and $\mathrm{Ni}_{3} \mathrm{Ti}$, were formed after a splitting of the precursor clusters. NiAl precipitates were found to contain a significant amount of $\mathrm{Fe}$ while $\mathrm{Ni}_{3} \mathrm{Ti}$ precipitates were $\mathrm{Fe}$-free [32]. It is generally believed that $\mathrm{NiAl}$ precipitates which are formed out of the solute-rich clusters have a high solubility of $\mathrm{Fe}$, thus the $\mathrm{Fe}$ atoms in precursor clusters tend to be retained in $\mathrm{NiAl}$ precipitates rather than in $\mathrm{Ni}_{3} \mathrm{Ti}$ precipitates [38][82]. Therefore, the presence of $\mathrm{Fe}$ atoms in the precipitates of Mn-based maraging steels in this study implies that the precipitate is more likely to be NiAl phase or similar phases with high $\mathrm{Fe}$ solubility, rather than the $\mathrm{Ni}_{3} \mathrm{Ti}$ phase in the conventional maraging steels. On the other hand, another possibility proposes that the existing of $\mathrm{Fe}$ and $\mathrm{Mn}$ in precipitates is associated with the lateral diffusion of Fe and $\mathrm{Mn}$ atoms from the matrix during aging [135]. Therefore, further investigation is needed to determine which viewpoint is more reliable.

In order to quantify the elemental constituents of precipitates, standardless quantitative analysis on at least 10 precipitates within each sample was undertaken and the results are summarized in Table 5-2. As discussed in Section 3.3.4.2, standardless quantitative analysis cannot provide accurate quantitative results, but given that the precipitate forming elements in this study are not trace and their atomic numbers are not small, the standardless quantitative results should be acceptable.

The data in Table 5-2 did not reveal significant variation in the chemical composition of precipitate among the three alloys. This suggests that the precipitates are quite chemically stable within the aging time up to $10080 \mathrm{~min}$. The $7 \% \mathrm{Mn}$ alloy is chosen to study the $\mathrm{Fe}$ evolution in precipitate in order to eliminate the interference from austenite reversion. In the $500{ }^{\circ} \mathrm{C} / 480 \mathrm{~min}$ state, 15.8 at.\% $\mathrm{Fe}$ is detected in the precipitate followed by a decrease to 9.8 at.\% Fe after aging for 2880 min. Further aging to $10080 \mathrm{~min}$ did not further change the Fe concentration which suggests that the $\mathrm{Fe}$ in precipitates had reached its equilibrium concentration after prolonged aging. Owing to the limitation of the TEM-EDS technique, compositional 
analysis was not carried out on the precipitates in the very beginning of aging process. But Millán et al. [135], who employed APT to measure the chemical composition of precipitates in a 9\% Mn maraging steel, reported the precipitates with an average composition of 35 at. $\% \mathrm{Ni}, 11$ at. $\% \mathrm{Mn}, 2$ at.\% Al, 2 at.\% Ti, and 50 at.\% $\mathrm{Fe}$ after aging for 0.5 hours. The evolution of $\mathrm{Fe}$ concentration in precipitates confirms our speculation that the $\mathrm{Fe}$ atoms in the precipitate are retained from the precursor clusters rather than via the diffusion from the Fe matrix during aging.

Owing to the partitioning of $\mathrm{Fe}$ and $\mathrm{Mn}$, the precipitates have a non-stoichiometric composition which can be described as $\mathrm{Ni}_{2} \mathrm{TiAl}$ or $\mathrm{Ni}(\mathrm{Ti}, \mathrm{Al})$ phase. This deviation from the stoichiometric composition due to the element substitution is proposed to increase the local disordering and dislocation climb or cutting, thereby enhancing the ductility of phases [103].

It is important to emphasize that in Raabe et al.'s APT study [135], the Al and Ti concentrations of precipitates were relatively low and these low levels maintained up to aging for $196 \mathrm{~h}$ which was contradictory to the present results. On the other hand, as the $\mathrm{Ni}$ and $\mathrm{Mn}$ concentrations in their study increased moderately with the aging time, the composition of the precipitates in their work was $\mathrm{Ni}_{44} \mathrm{Fe}_{20} \mathrm{Mn}_{24} \mathrm{Al}_{6} \mathrm{Ti}_{4}$ which, in their opinion, was more close to $\mathrm{Ni}_{50}(\mathrm{Mn}, \mathrm{Al}, \mathrm{Ti})_{50}$. 
Table 5-2 Average chemical composition in at.\% of the nano-sized precipitates evolved at $500{ }^{\circ} \mathrm{C}$ measured by TEM-EDS.

\begin{tabular}{|c|c|c|c|c|c|c|}
\hline & & Al-K & Ti-K & $\mathrm{Mn}-\mathrm{K}$ & $\mathrm{Fe}-\mathrm{K}$ & $\mathrm{Ni}-\mathrm{K}$ \\
\hline \multirow[t]{3}{*}{$7 \% \mathrm{Mn}$} & $480 \mathrm{~min}$ & $30.2 \pm 1.4$ & $17.4 \pm 1.0$ & $4.3 \pm 0.6$ & $15.8 \pm 0.6$ & $32.4 \pm 0.8$ \\
\hline & $2880 \mathrm{~min}$ & $33.1 \pm 1.2$ & $17.2 \pm 1.3$ & $5.6 \pm 0.5$ & $9.8 \pm 0.6$ & $34.3 \pm 0.9$ \\
\hline & $10080 \mathrm{~min}$ & $33.4 \pm 0.4$ & $14.9 \pm 0.5$ & $7.1 \pm 0.5$ & $9.8 \pm 0.3$ & $35.0 \pm 0.3$ \\
\hline \multirow[t]{3}{*}{$10 \% \mathrm{Mn}$} & $480 \mathrm{~min}$ & $30.4 \pm 0.5$ & $15.6 \pm 0.8$ & $6.5 \pm 0.6$ & $14.7 \pm 0.4$ & $32.8 \pm 0.8$ \\
\hline & $2880 \mathrm{~min}$ & $33.0 \pm 0.7$ & $17.7 \pm 0.6$ & $5.4 \pm 0.5$ & $12.2 \pm 0.4$ & $31.8 \pm 0.7$ \\
\hline & $10080 \mathrm{~min}$ & $33.5 \pm 0.7$ & $18.1 \pm 0.9$ & $4.7 \pm 0.5$ & $13.4 \pm 1.3$ & $30.3 \pm 1.5$ \\
\hline \multirow[t]{3}{*}{$12 \% \mathrm{Mn}$} & $480 \mathrm{~min}$ & $30.2 \pm 0.9$ & $15.6 \pm 1.1$ & $6.7 \pm 0.4$ & $15.1 \pm 0.9$ & $32.5 \pm 1.1$ \\
\hline & $2880 \mathrm{~min}$ & $32.9 \pm 0.2$ & $16.8 \pm 0.5$ & $5.7 \pm 0.4$ & $12.2 \pm 0.3$ & $32.5 \pm 0.5$ \\
\hline & $10080 \mathrm{~min}$ & $33.4 \pm 0.8$ & $17.3 \pm 1.1$ & $5.4 \pm 0.9$ & $12.7 \pm 1.0$ & $31.2 \pm 1.3$ \\
\hline
\end{tabular}




\subsection{Crystal structure of precipitates}

\subsubsection{Determination of crystal structure}

According to the compositional study, the intermetallic precipitates in Mn-based maraging steels may be $\mathrm{Ni}_{2} \mathrm{TiAl}$ or $\mathrm{Ni}(\mathrm{Al}, \mathrm{Ti})$. XRD analyses of the aged samples (see Section 4.3) did not reveal any diffraction signal of the precipitates even when the microstructural observation clearly showed a significant amount of precipitates, which further suggests that the precipitates are highly likely to be $\mathrm{B} 2-\mathrm{Ni}(\mathrm{Al}, \mathrm{Ti})$ or $\mathrm{L} 2{ }_{1}-\mathrm{Ni}_{2} \mathrm{TiAl}$ phase as most of their diffraction peaks overlap with the peaks of $\alpha^{\prime}$ martensite matrix. Since the crystallographic constitution of the phase determines its electron diffraction behaviour which plays an important role in the precipitate characterization, the crystallographic details of $\mathrm{B} 2-\mathrm{NiAl}$ and $\mathrm{L}_{2}-\mathrm{Ni}_{2} \mathrm{TiAl}$ are discussed.

- B2-ordered NiAl

Figure 5-8 displays the crystal structure of B2-NiAl phase and its simulated electron diffraction patterns along low-index zone axis directions. The B2 phase has a $\mathrm{CsCl}-$ type crystal structure. It is constituted by two primitive cubic lattices which are stacked alternatively along the vector $1 / 2<111\rangle$ [17]. This structure can also be described as a body-centred cubic (bcc) lattice of $\mathrm{Ni}$ with the centre positions being replaced by $\mathrm{Al}$ atoms. The lattice parameter of the stoichiometric composition is $0.2887 \mathrm{~nm}$ which is very close to that of $\alpha^{\prime}$-martensite $(0.2884 \mathrm{~nm})$ (see Appendix). 
(a)
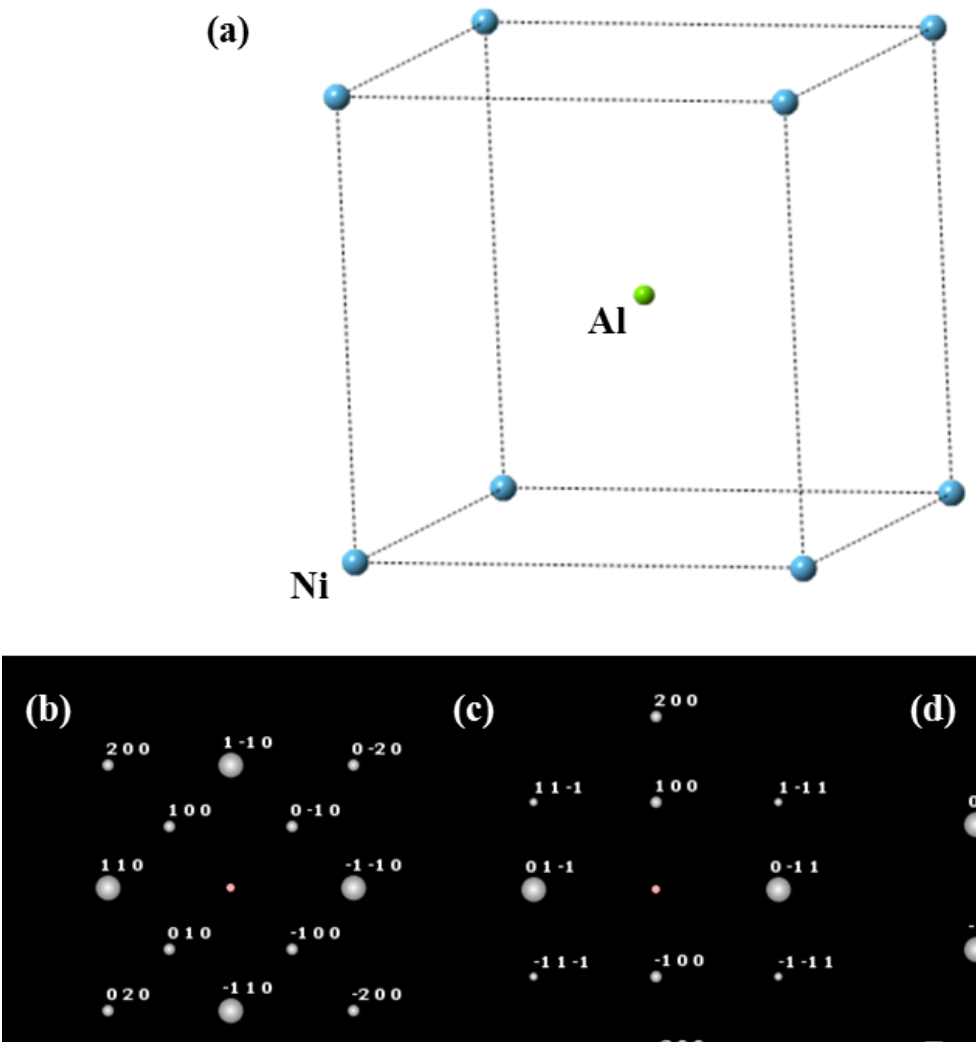

(c)

200
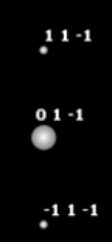

[011]
100

-

$-100$

$8^{200}$ (d)

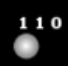

$0^{0} 1-1$

0.11

$i^{-111}$

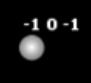

$\bullet$

$8^{-11}$

$0^{1 \cdot 10}$

$[\overline{1} 11]$

Figure 5-8 Crystal structure of the B2-ordered NiAl phase and simulated electron diffraction patterns along low-index zone axis directions simulated using ICDD PDF-4+ software.

- L21-ordered $\mathrm{Ni}_{2} \mathrm{TiAl}$

The $\mathrm{L} 2{ }_{1}$ structure simply involves a further ordering of the $\mathrm{Al}$ and $\mathrm{Ti}$ atoms on the Al sublattice in B2 structure. The structure can be regarded as possessing three sets of sublattice (Figure 5-9(a)), among which two interpenetrating face centred cubic lattices are occupied by $\mathrm{Al}$ and $\mathrm{Ti}$ atoms respectively and a primitive cubic lattice of $\mathrm{Ni}$ atoms is shifted by the vector $1 / 4<111>$. Figure $5-10$ (a) is an equivalent way to illuminate the $\mathrm{L} 2{ }_{1}$ structure but easier to understand. The unit cell is built up by eight small bcc lattices. The corners of each small cubic lattice are occupied by $\mathrm{Ni}$ atoms with the centre positions being orderly occupied by $\mathrm{Al}$ and $\mathrm{Ti}$ atoms. Thus, two different types of B2 structure are arranged successively in each direction. The lattice parameter of the small body centred cubic lattice $(0.295 \mathrm{~nm})$ is half that of the 
entire unit cell and is close to that of B2-NiAl phase $(0.2887 \mathrm{~nm})$. When the centre positions are fully occupied by one type of atom, for example $\mathrm{Al}$, the phase is transformed to $\mathrm{B} 2$-ordered NiAl phase; when the $\mathrm{Al}$ and $\mathrm{Ti}$ atoms are randomly arranged, the phase is degraded to $\mathrm{B} 2$-ordered $\mathrm{Ni}(\mathrm{Al}, \mathrm{Ti})$ phase.

(a)
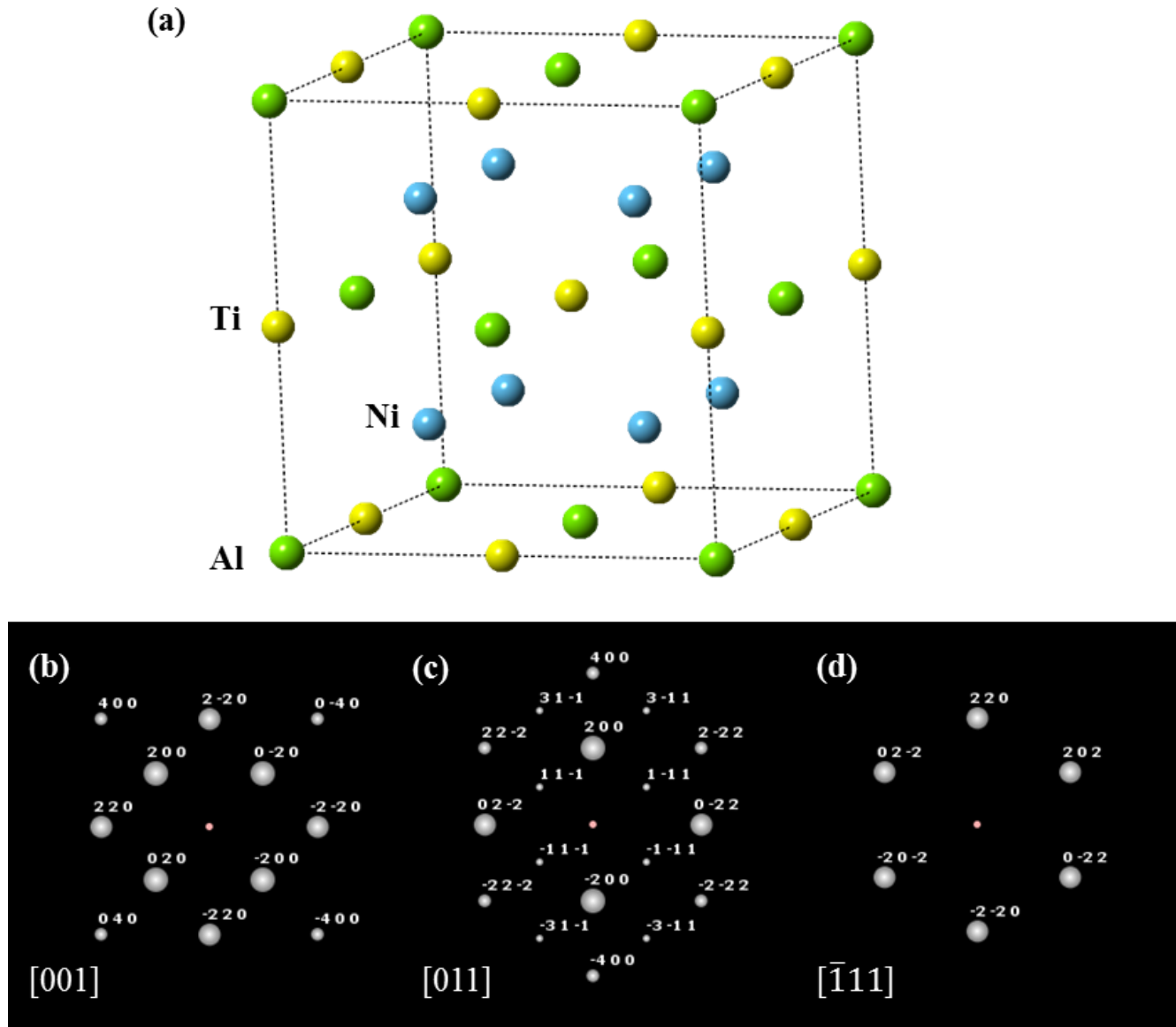

Figure 5-9 Crystal structure of the $\mathrm{L}_{1}$-ordered $\mathrm{Ni}_{2} \mathrm{TiAl}$ phase and its simulated electron diffraction patterns along low-index zone axis directions simulated using ICDD PDF-4+ software.

The phase with the fully ordered $\mathrm{L} 2_{1}$ structure is named as Heusler phase. The Heusler phases become strongly ferromagnetic after quenching from $800{ }^{\circ} \mathrm{C}$ [220]. The structure is body centred cubic but with a face centred superlattice, as illustrated in the electron diffraction patterns (Figure 5-9(b), (c) and (d)). Comparing their standard electron diffraction pattern, it is found that the B2 and $\mathrm{L} 22_{1}$ structures have 
similar diffraction patterns along the [001] and [111] zone axes. In the [001] zone axis, the $\{220\}$ and $\{002\}$ reflections of $\mathrm{L}_{1}$-ordered $\mathrm{Ni}_{2} \mathrm{TiAl}$ coincide with the $\{110\}$ and $\{001\}$ reflections of B2-ordered NiAl, respectively (Figure 5-8(b) and Figure $5-9(b))$. In the $[\overline{1} 11]$ zone axis, the $\{220\}$ reflections of $\mathrm{L}_{1}$-ordered $\mathrm{Ni}_{2} \mathrm{TiAl}$ coincide with the $\{110\}$ reflections of B2-ordered NiAl (Figure 5-8(d) and Figure 5-9(d)). Although the theoretical intensity difference exists between the two phases, it is difficult to distinguish it under the real beam condition. The characteristic reflections to distinguish the two phases are only visible along the [011] zone axis. Figure 5-10 presents the electron diffraction patterns of both $\mathrm{L}_{1}$-ordered $\mathrm{Ni}_{2} \mathrm{TiAl}$ and B2-ordered NiAl along the [011] zone axis for a better comparison. The $\{222\}$ and $\{002\}$ reflections of $\mathrm{L}_{2}{ }_{1}$-ordered $\mathrm{Ni}_{2} \mathrm{TiAl}$ coincide with the $\{111\}$ and $\{001\}$ reflections of B2-ordered NiAl, whereas the $\{111\}$ and $\{311\}$ reflections exclusively exist in the $\mathrm{L}_{1}$-ordered $\mathrm{Ni}_{2} \mathrm{TiAl}$ phase. Therefore, the $\{111\}$ and $\{311\}$ reflections along the [011] zone axis are considered as the characteristic diffraction spots for L2 1 structure. 
Chapter 5 Characterization of $\mathrm{L2}_{1}$-ordered $\mathrm{Ni}_{2} \mathrm{TiAl}$ intermetallic phase and its precipitation behaviour

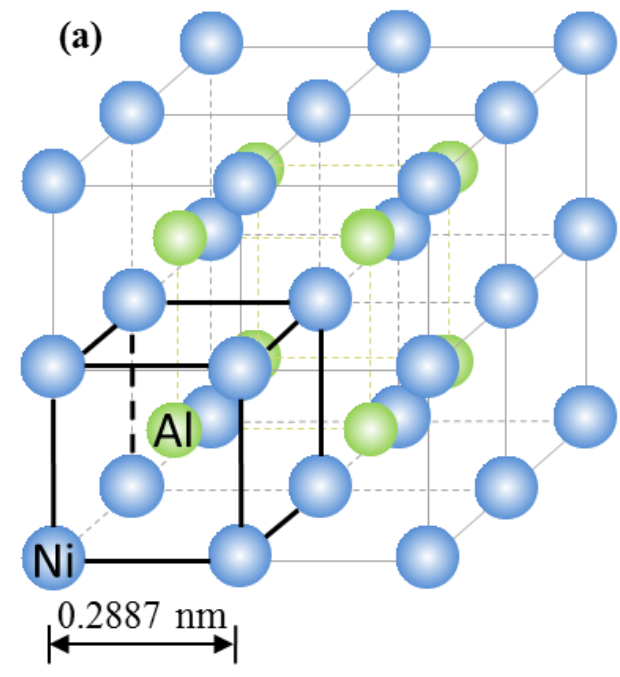

NiAl (B2)

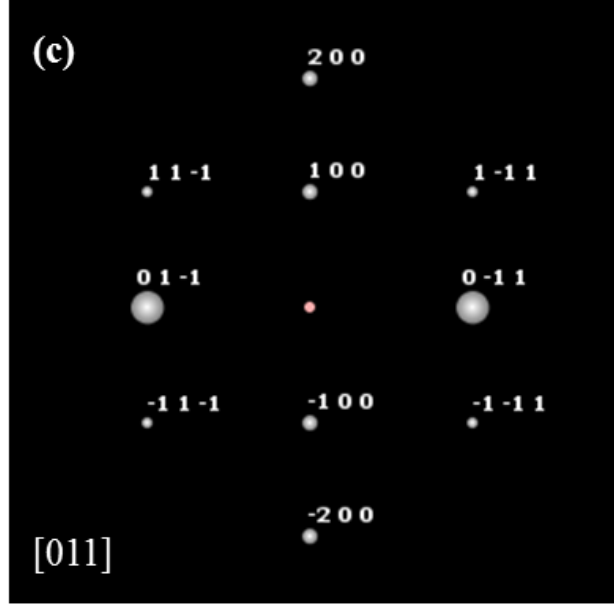

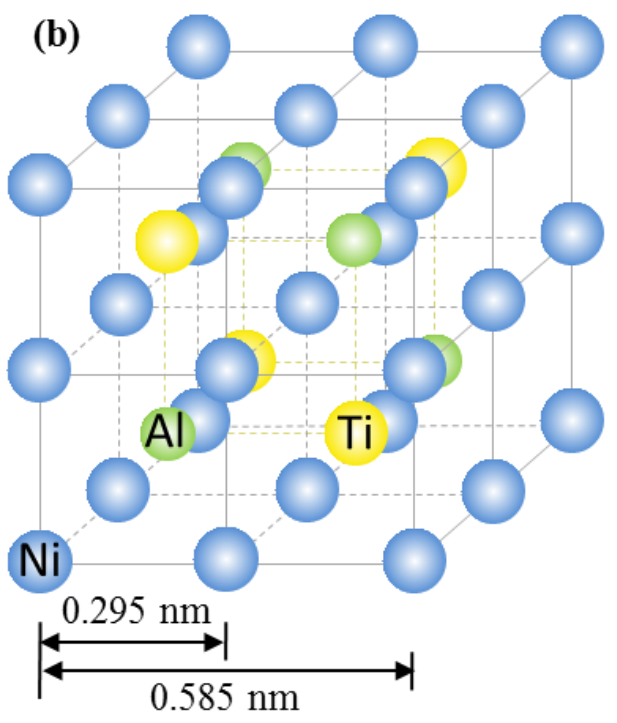

$\mathrm{Ni}_{2} \mathrm{TiAl}\left(\mathrm{L} 2_{1}\right)$

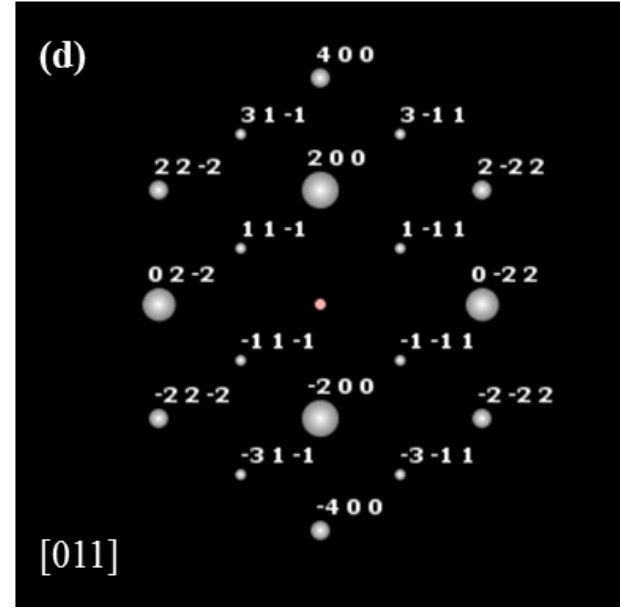

Figure 5-10 (a) Eight unit cells of the B2-ordered NiAl for a better comparison with (b) the unit cell of $\mathrm{L} 2{ }_{1}$-ordered $\mathrm{Ni}_{2} \mathrm{TiAl}$; diffraction patterns along the [011] zone axis of (c) B2ordered $\mathrm{NiAl}$ and (d) $\mathrm{L} 2{ }_{1}-\mathrm{Ni}_{2} \mathrm{TiAl}$ simulated using ICDD PDF-4+ software.

A HREM study was carried out to identify the crystal structure of the precipitates. Figure 5-11 shows the HREM micrographs of precipitate along the [011] and [111] zone axes, respectively. To better clarify the crystalline structure of the precipitate, fast Fourier transform (FFT) analysis is displayed as inset. The characteristic $\{111\}$ reflections along the [011] zone axis of $\mathrm{L}_{1}$-ordered $\mathrm{Ni}_{2} \mathrm{TiAl}$ phase are indexed (see inset). Figure 5-11(b) and (d) reveal the inverse fast Fourier transform (IFFT) image by removing the background noise. The atomic arrangement is coincident with that 
Chapter 5 Characterization of $\mathrm{L2}_{1}$-ordered $\mathrm{Ni}_{2} \mathrm{TiAl}$ intermetallic phase and its precipitation behaviour

of $\mathrm{Ni}_{2} \mathrm{TiAl}$; the indexed d-spacing of $0.3335 \mathrm{~nm}$ for the $\{111\}$ planes and $0.206 \mathrm{~nm}$ for the $\{220\}$ planes are very close to the standard d-spacing of $\mathrm{Ni}_{2} \mathrm{TiAl}$ phase (0.3377 $\mathrm{nm}$ for $\{111\}$ plane and $0.2068 \mathrm{~nm}$ for $\{220\}$ plane) given by the ICCD database. The slight deviation may be associated with either the non-stoichiometric composition or the error from the measurement and/or equipment.
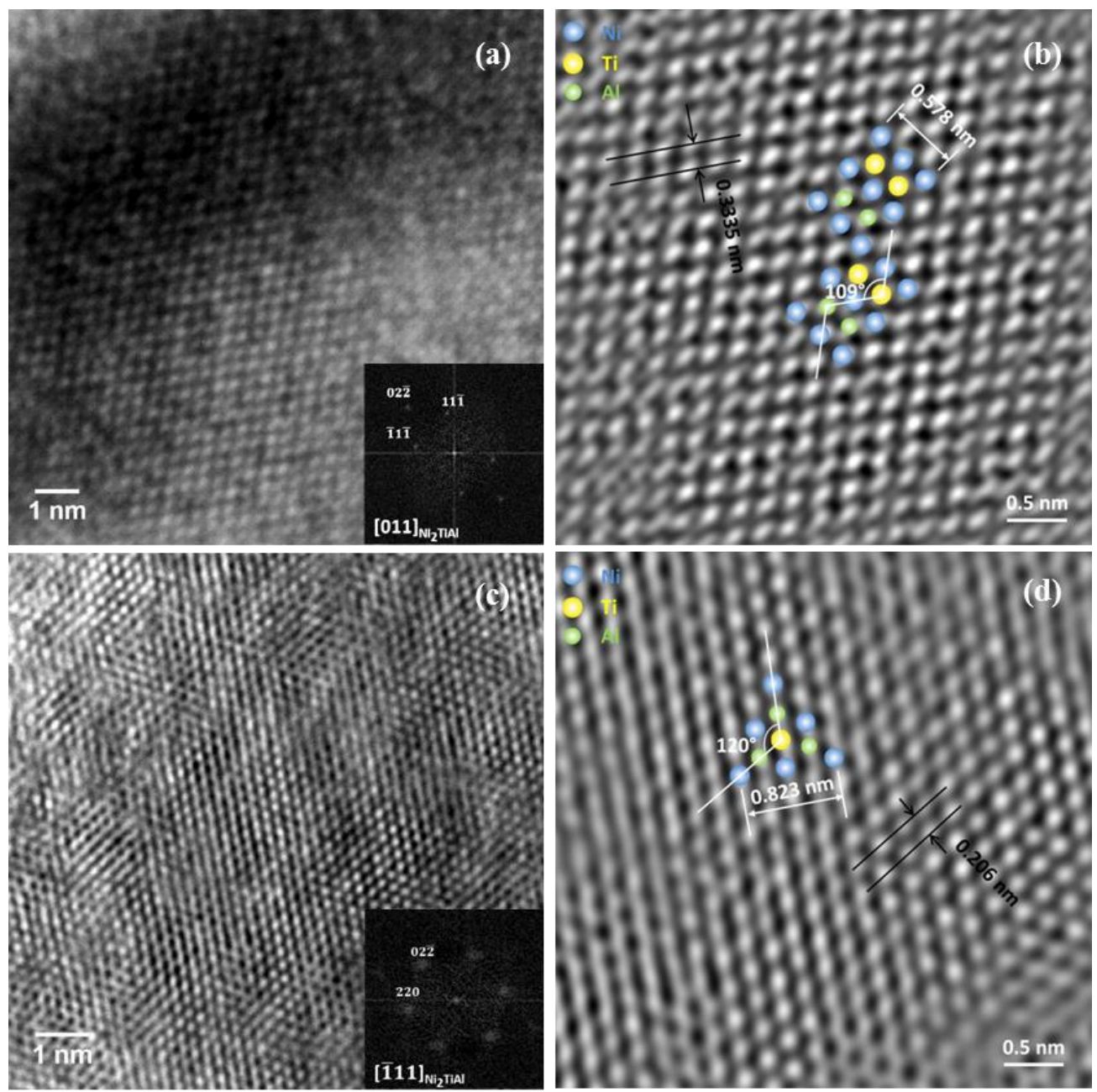

Figure 5-11 HREM micrographs of precipitates in $12 \% \mathrm{Mn}$ alloy aged at $500{ }^{\circ} \mathrm{C}$ for 10080 min, (a) [011] zone axis; (b) IFFT of (a); (c) [1111] zone axis; (d) IFFT of (c).

More information about the crystal structure is obtained by SAED analyses (Figure 5-12). The $\{111\}$ and $\{311\}$ reflections along the [011] zone axis, which are unique to the $\mathrm{L} 2_{1}$ structure, are displayed in the inset of Figure 5-12(b). The presence of $\mathrm{L} 2_{1}$-ordered $\mathrm{Ni}_{2} \mathrm{TiAl}$ phase in the dark-field images (white spherical phase in Figure 
Chapter 5 Characterization of $\mathrm{L2}_{1}$-ordered $\mathrm{Ni}_{2} \mathrm{TiAl}$ intermetallic phase and its precipitation behaviour

5-12(b)), which was acquired by using the (11) $)$ superlattice spots, further confirms the precipitate is, or at least consists of, $\mathrm{L} 2{ }_{1}$-ordered $\mathrm{Ni}_{2} \mathrm{TiAl}$ phase.
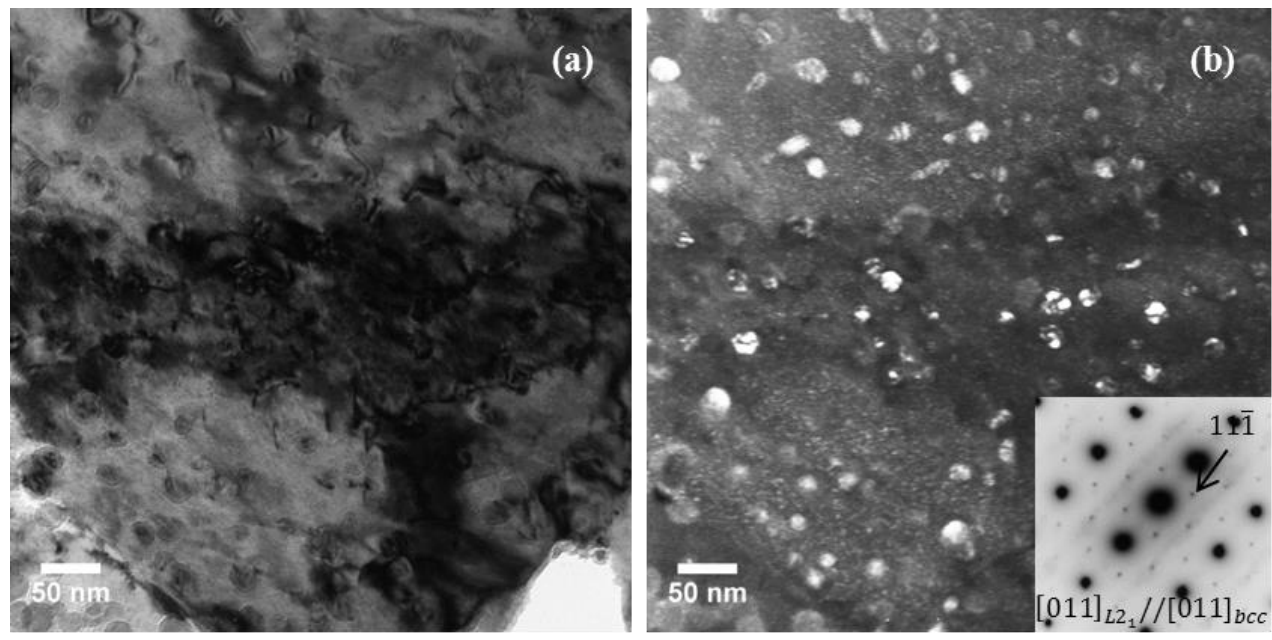

Figure 5-12 (a) Bright-field TEM micrograph of 7\% Mn alloy aged at $500{ }^{\circ} \mathrm{C}$ for $10080 \mathrm{~min}$ along [011] zone axis and (b) corresponding dark-field micrograph using the [11 $\overline{1}]$ superlattice spots of precipitates (inset is the corresponding diffraction pattern).

\subsubsection{The orientation relationship between the precipitate and martensite matrix}

Representative selected area diffraction patterns (SADPs) along low-index zone axis directions (10\% Mn alloy, $500{ }^{\circ} \mathrm{C} / 10080$ min state) are shown in Figure 5-13.

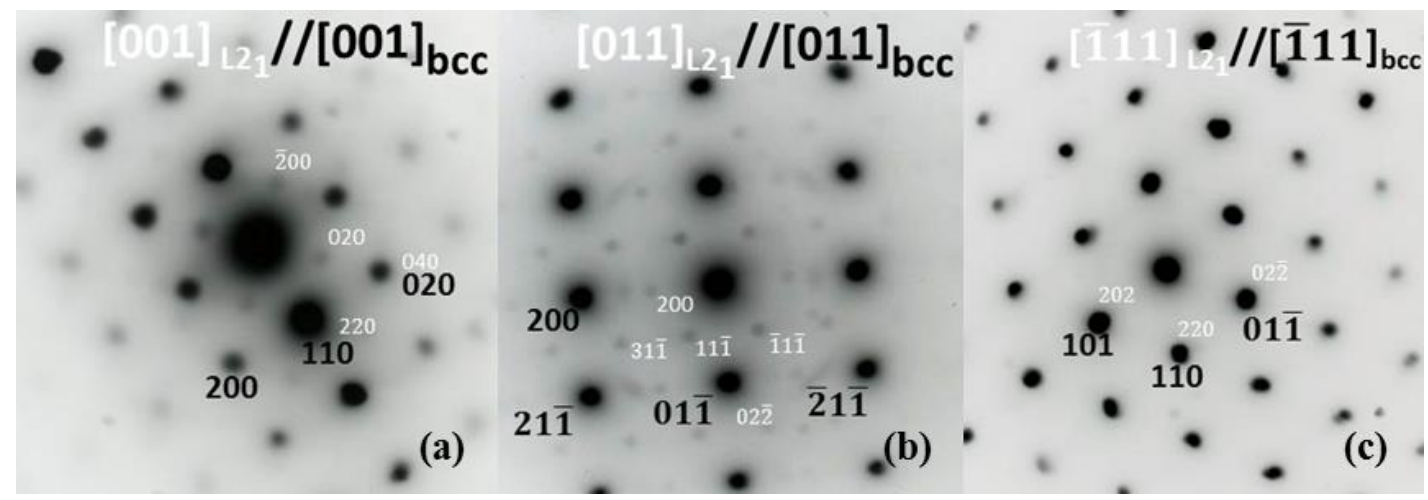

Figure 5-13 Selected area diffraction patterns taken on thin foil samples $\left(500{ }^{\circ} \mathrm{C} / 10080 \mathrm{~min}\right.$, 10\% Mn alloy) along (a) [001], (b) [011] and (c) [111] zone axes. 
The coherency between precipitates and martensite matrix was investigated. The SADPs reveal that the orientation of $\mathrm{L}_{1}$-ordered $\mathrm{Ni}_{2} \mathrm{TiAl}$ precipitate is exactly coincident with that of the $\alpha^{\prime}$-martensite matrix (Figure 5-13). In both the [001] and [011] zone axes (Figure 5-13(a) and (b)), the $\{040\}$ and $\{220\}$ reflections of L2 $1^{-}$ $\mathrm{Ni}_{2} \mathrm{TiAl}$ phase are overlapping with the $\{020\}$ and $\{110\}$ reflections of the bcc matrix, respectively; in the $[\overline{1} 11]$ zone axis, the diffraction pattern of $\mathrm{Ni}_{2} \mathrm{TiAl}$ exactly matches that of the matrix, so no extra spots originating from the precipitate were observed (Figure 5-13(c)).

The lattice parameter of $\mathrm{L} 2{ }_{1}$-ordered $\mathrm{Ni}_{2} \mathrm{TiAl}$ phase was determined according to the SADPs. The camera length was calibrated using the lattice parameter of $\alpha^{\prime}$ martensite matrix obtained from the XRD analysis. Based on this calibration, the lattice parameter of the $\mathrm{L} 2$-ordered $\mathrm{Ni}_{2} \mathrm{TiAl}$ phase was calculated as $0.5819 \mathrm{~nm}$. Half of the lattice parameter of $\mathrm{L} 2_{1}$-ordered $\mathrm{Ni}_{2} \mathrm{TiAl}$, which is the dimension of the small cubic lattice in Figure 5-10(b), is close to the lattice parameter of $\alpha^{\prime}$-martensite matrix. The misfit between them is calculated as

$$
\delta=\frac{2 \times\left|\frac{1}{2} \mathrm{a}_{\mathrm{Ni}_{2} \mathrm{TiAl}}-\mathrm{a}_{\mathrm{bcc}}\right|}{\frac{1}{2} \mathrm{a}_{\mathrm{Ni}_{2} \mathrm{TiAl}}+\mathrm{a}_{\mathrm{bcc}}} \times 100 \%=1.24 \%
$$

where $\mathrm{a}_{\mathrm{Ni}_{2} \mathrm{TiAl}}$ is the lattice parameter of $\mathrm{Ni}_{2} \mathrm{TiAl}$ and $\mathrm{a}_{\mathrm{bcc}}$ is the lattice parameter of the martensitic matrix measured by XRD. Owing to the small misfit of the lattice parameters and the special orientation relationship between the two phases, it is proposed that the $\mathrm{L} 2{ }_{1}$-ordered $\mathrm{Ni}_{2} \mathrm{TiAl}$ precipitate is coherent and coplanar with the martensite matrix. Figure 5-14 presents the nano-scale precipitates were uniformly dispersed in martensite matrix in both the $7 \% \mathrm{Mn}$ and $10 \% \mathrm{Mn}$ alloys aged at $500^{\circ} \mathrm{C}$ for $10080 \mathrm{~min}$. The dumbbell-shaped precipitates reveal that they remain coherent with the matrix even after aging at $500{ }^{\circ} \mathrm{C}$ for 10080 min, according to AshbyBrown contrast theory [221]. 
Chapter 5 Characterization of $\mathrm{L2}_{1}$-ordered $\mathrm{Ni}_{2} \mathrm{TiAl}$ intermetallic phase and its precipitation behaviour
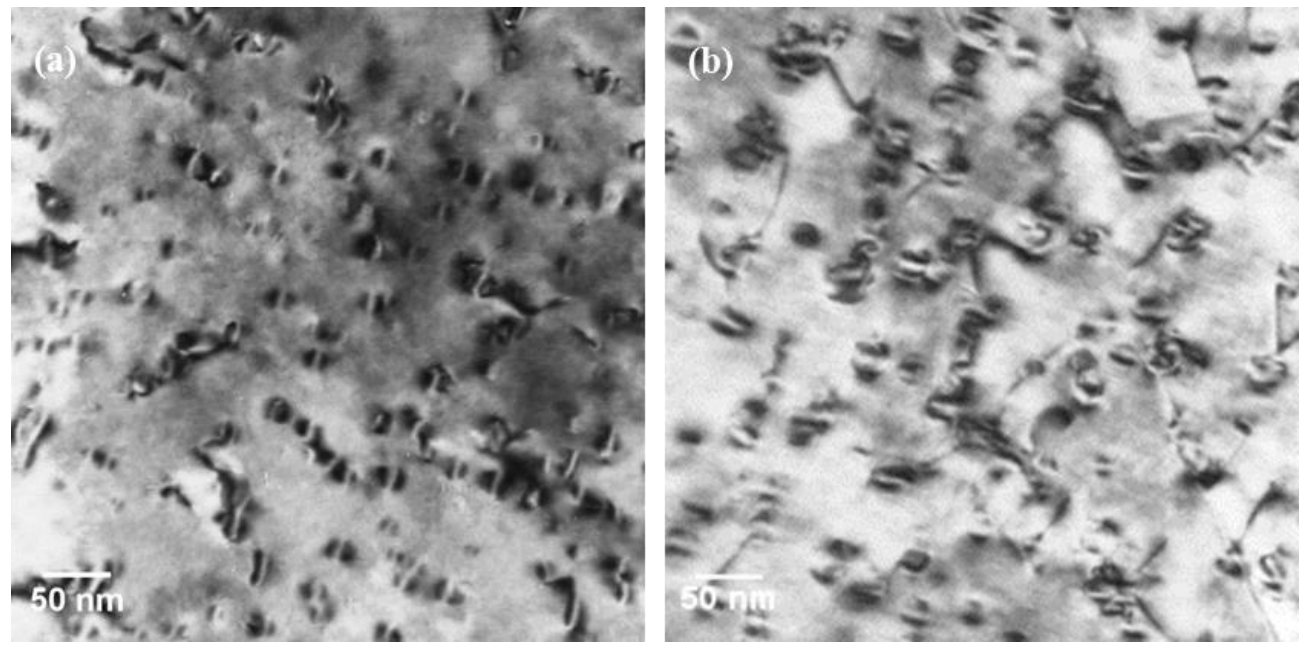

Figure 5-14 Two-beam bright field micrographs of (a) 7\% Mn and (b) 10\% Mn alloys after aging at $500{ }^{\circ} \mathrm{C}$ for $10080 \mathrm{~min}$.

\subsection{Core/shell precipitates}

Figure 5-15 shows the representative bright-field TEM micrographs (left column) of precipitates formed when aging at $500{ }^{\circ} \mathrm{C}$ for $10080 \mathrm{~min}$ in $7 \% \mathrm{Mn}$ alloy (Figure 5-15(a)), 10\% Mn alloy (Figure 5-15(b)) and 12\% Mn alloy (Figure 5-15(c)), respectively. Corresponding high-magnification TEM micrographs obtained from carbon extraction replicas are correspondingly displayed in the right column. As shown in Figure 5-15, precipitates with a well-developed core/shell structure can be distinguished by the darker central region surrounded by a periphery with brighter contrast. For the $7 \% \mathrm{Mn}$ alloy aged at $500{ }^{\circ} \mathrm{C}$ for $10080 \mathrm{~min}$ (Figure 5-15(a)), the average size of precipitates ranges from less than $10 \mathrm{~nm}$ to $\sim 20 \mathrm{~nm}$ with shells being 2-3 nm thick. Similar microstructure is found in the $10 \% \mathrm{Mn}$ alloy aged at $500{ }^{\circ} \mathrm{C}$ for $10080 \mathrm{~min}$ (Figure 5-15(b)). The thickness of the shell is approximately constant at 2-3 nm despite the sizes of precipitates varying from $12 \mathrm{~nm}$ to $24 \mathrm{~nm}$, which indicates that the thickness of the shell did not increase with the growth of the core. 
Chapter 5 Characterization of $\mathrm{L2}_{1}$-ordered $\mathrm{Ni}_{2} \mathrm{TiAl}$ intermetallic phase and its precipitation behaviour

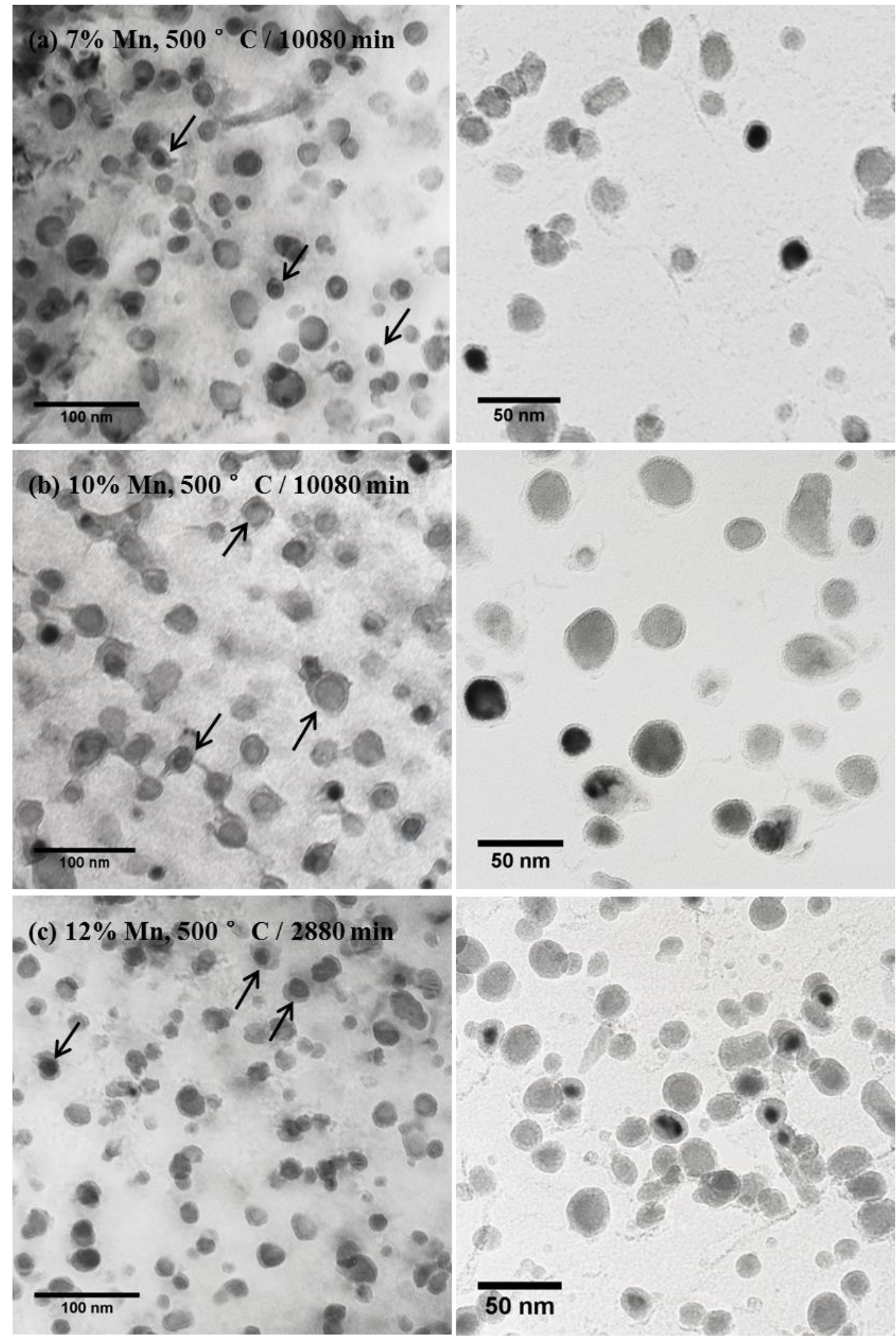

Figure 5-15 Left column: representative bright-field TEM micrographs. Right column: magnified carbon extraction replica TEM micrographs of precipitates. (a) 7\% Mn alloy, $500{ }^{\circ} \mathrm{C} / 10080 \mathrm{~min}$; (b) $10 \% \mathrm{Mn}$ alloy, $500{ }^{\circ} \mathrm{C} / 10080 \mathrm{~min}$ and (c) $12 \% \mathrm{Mn}$ alloy, $500{ }^{\circ} \mathrm{C} /$ $2880 \mathrm{~min}$. 
As shown in the HAADF micrograph (Figure 5-16), the contrast variation within each precipitate is evident. HAADF image was taken under conditions excluding any diffraction effect, and the image contrast under HAADF mode is $Z$ contrast which depends on the mass and thickness. For the thickness contrast, the following equation describes the relationship between the beam intensity detected by camera, $I^{\prime}$, and the thickness of the object, $t$ [222]:

$$
I^{\prime}=I_{0}(1-\exp (-\mu t))
$$

where $I_{0}$ is the beam intensity before it enters the object and $\mu$ is the attenuation coefficient. If the object is spherical like the precipitate in this study, the beam intensity detected $I^{\prime}$ should be continuous along the diameter. The mass contrast, on the other hand, is demonstrated to be roughly proportional to the square of atomic number [223]. Therefore, the sharp contrast at the periphery should be correlated to the mass contrast.

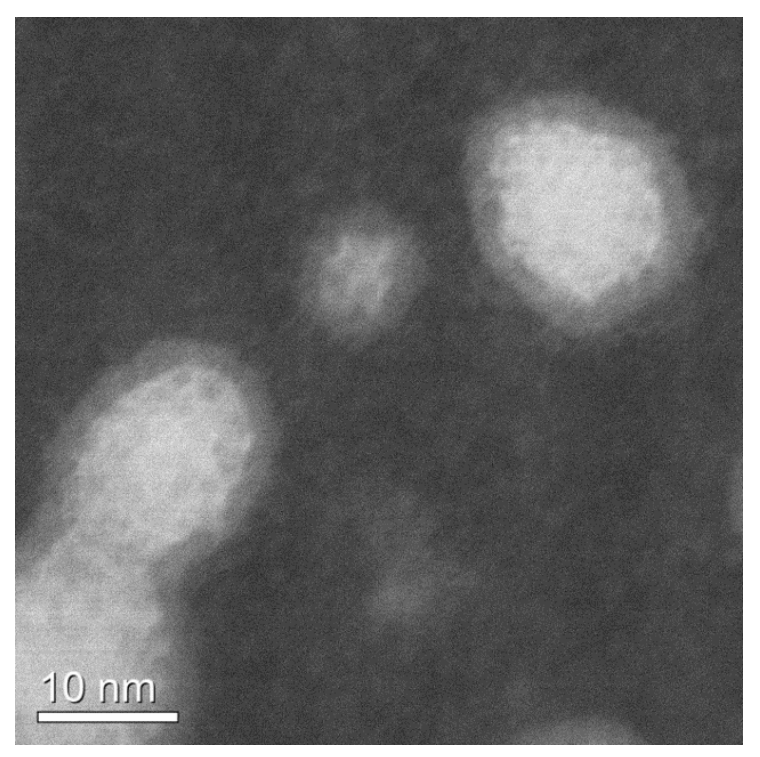

Figure 5-16 HAADF image of core/shell precipitates in $12 \% \mathrm{Mn}$ alloy aged at $500{ }^{\circ} \mathrm{C}$ for $2880 \mathrm{~min}$.

HREM micrographs of precipitates on carbon extraction replicas were acquired to study the core/shell structure. Precipitates with evident core/shell structure can be observed in Figure 5-17(a). Although the interface is visible between the core and shell, the shell is found coherent and coplanar with the core (Figure 5-17(b)). A 
magnified HREM micrograph at the periphery of precipitate (Figure 5-17(c)) suggests that the precipitate is one single crystal. The lattice structure is uniform despite a slight distortion at the smearing core/shell interface.

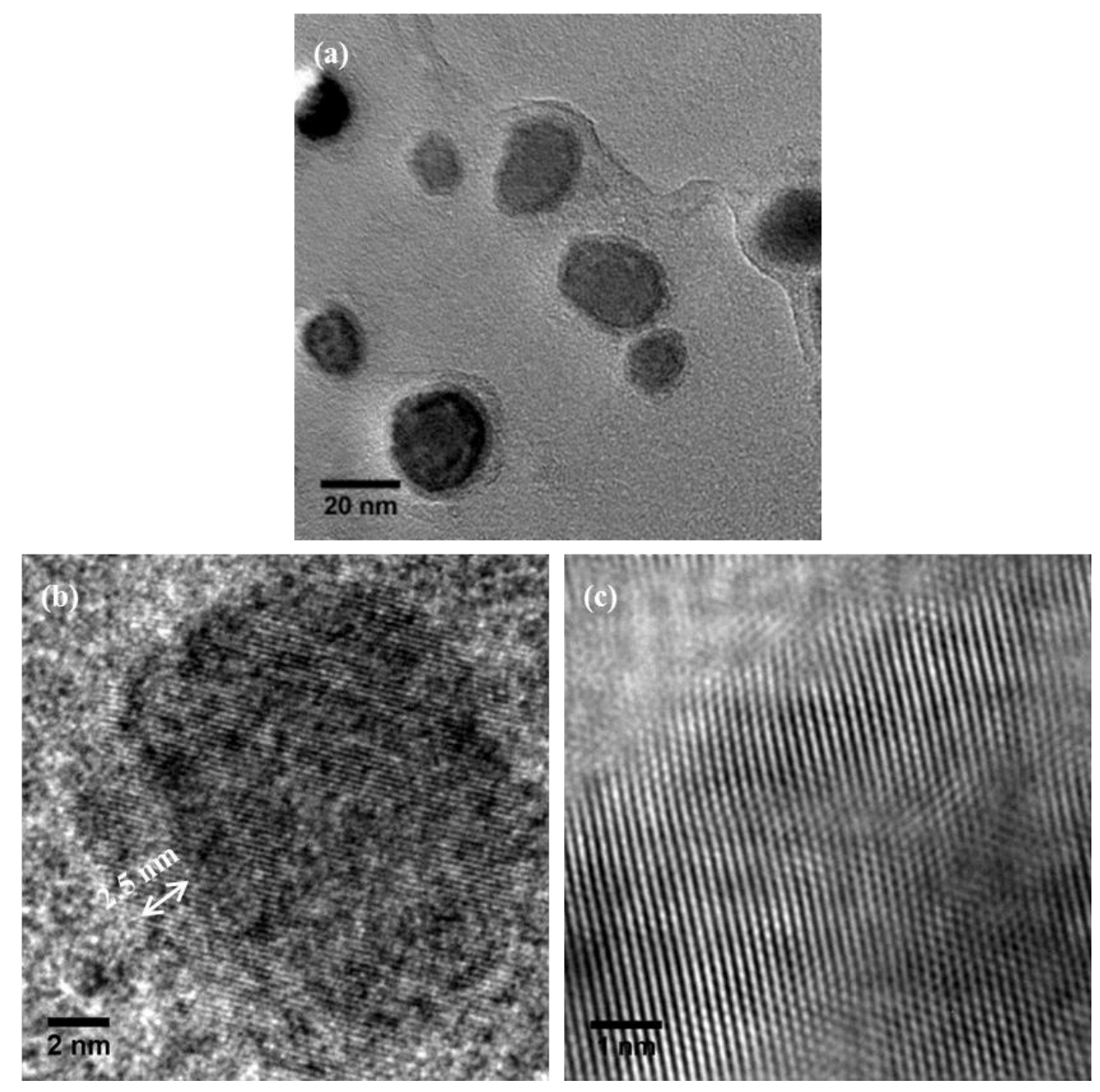

Figure 5-17 HREM micrographs of the precipitate in $12 \% \mathrm{Mn}$ alloy aged at $500{ }^{\circ} \mathrm{C}$ for 10080 min. (a) The low-magnification images of precipitates, (b) core/shell structure at a higher magnification and (c) lattice information at the periphery of precipitate.

The discussion on the non-stoichiometric composition of precipitates (especially the high $\mathrm{Al}$ concentration) in Section 5.5 implies the precipitates may be constituted by more than one phase. The existence of the core/shell structure in precipitates is another evidence for this speculation. It is proposed that the precipitate may possess a $\mathrm{NiAl} / \mathrm{Ni}_{2} \mathrm{TiAl}$ structure. As discussed in Section 5.6.1, these two phases have very similar crystal structure, so they can be regarded as one single crystal. As the spherical phase in the dark-field micrograph in Figure 5-12 was acquired by using 
the characteristic (11) superlattice spot of $\mathrm{L} 22_{1}$ structure, and the brighter core in HAADF micrograph (Figure 5-7) corresponds to a Ti-rich area of the precipitate as $\mathrm{Ti}$ has a higher atomic number than $\mathrm{Al}\left({ }^{22} \mathrm{Ti}\right.$ vs. $\left.{ }^{13} \mathrm{Al}\right)$, we further propose that the core should be $\mathrm{L} 2{ }_{1}-\mathrm{Ni}_{2} \mathrm{TiAl}$ phase and the shell should be B2-NiAl. A further investigation is required to examine the core/shell structure within precipitate.

\subsection{Discussion}

\subsubsection{The effects of volume fractions on the coarsening behaviour}

- Precipitate size distribution (PSD)

PSDs provide us a pathway to estimate the goodness of fit of the LSW theory to the precipitate coarsening. As discussed in Section 2.3.3, LSW model is strictly valid under the assumption that the volume fraction of precipitates $\left(f_{p}\right)$ is approaching zero and time-invariant. Modern theoretical and simulation studies, however, revealed a discrepancy between the LSW theory and experiment due to $f_{p} \neq 0$ in reality [224][225]. According to the modified LSW theories, the PSDs are predicted to broaden rapidly when $f_{p}$ is low and approach the distribution model for interfacedominated coarsening when the limit $f_{p} \rightarrow 1$ [224]. The TK model (Tokuyama and Kawasaki, [226]) includes a correction for $f_{p}$ leading to the broadening in PSD function and decrease in the peak height compared to those of LSW model (see Figure 5-18). The results of TK model are in good agreement with LSW theory in the limit $f_{p} \rightarrow 0$ [136]. 


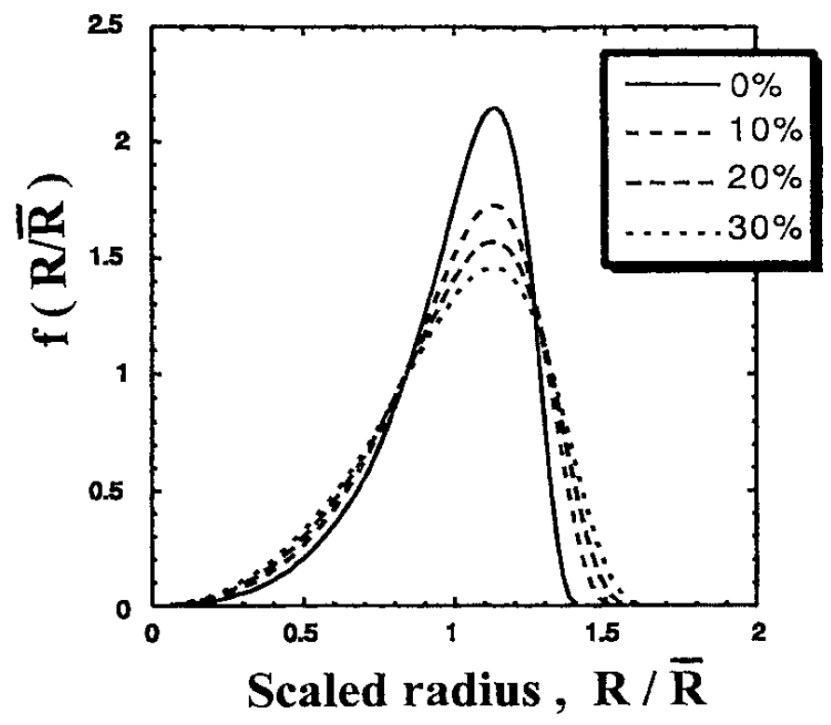

Figure 5-18 The precipitate size distribution as a function of reduced precipitate radius $R / \bar{R}$ for different volume fractions of precipitate $f_{p}$ [226].

The PSDs of the three alloys in different aging conditions were constructed to demonstrate the evolution of PSDs with aging time. As shown in Figure 5-3, the experimental PSD in the $500{ }^{\circ} \mathrm{C} / 480$ min state follows the LSW theory which indicates that $f_{p}$ can be roughly regarded as zero. After that the width of PSDs grew significantly $\left(500{ }^{\circ} \mathrm{C} / 2880 \mathrm{~min}\right.$ and $500{ }^{\circ} \mathrm{C} / 10080 \mathrm{~min}$ state) and the PSDs were more consistent with the TK model.

Apart from the volume fraction of precipitates, other attempts were also made to relate this discrepancy to the misfit of lattice parameter [224][227][228]. In this study, as the misfit of lattice parameters between phases has been demonstrated to be small, the broadening of the PSDs is not likely to be associated with the lattice misfit.

- Precipitate coarsening

Precipitate coarsening is controlled by either interface reaction or lattice diffusion, or sometimes by the combination of both. Generally the interface-dominated coarsening occurs at small size while the diffusion-dominated coarsening occurs at larger size. A combination of the two mechanisms takes effect at the transition stage. Experimental results in Section 5.4.2 reveal that at the early aging stage at $500{ }^{\circ} \mathrm{C}$, the precipitates exhibited an interface-dominated growth (coarsening) kinetics 
followed by a decrease in the growth rate indicating the initiation of the diffusiondominated coarsening kinetics (Figure 5-4). The coarsening evolution of precipitate size obeys the diffusion-controlled coarsening kinetics predicted by LSW theory (Figure 5-5). In contrast to the significant broadening of PSDs with the increase of $f_{p}$, experimental observation revealed that the dependence of coarsening rate constant on $f_{p}$ was small and the diffusion-controlled coarsening kinetic form $\bar{r}^{3} \sim K t$ can be maintained even when $f_{p}$ was high.

Many efforts have been made to investigate the effects of precipitated volume fraction on the coarsening kinetics and the shape of PSDs. Based on these studies, modified LSW theories, in which the precipitated volume fraction is taken into consideration, are established for the realistic case. All the modified theories agree that the non-zero volume fraction does not change the exponents of the temporal laws given by LSW (Equation (2.4), (2.5) and (2.6)) or the time-irrelevance of PSDs, but does change the amplitudes of the temporal power laws and the shapes of PSDs [136][137][138][139]. The presence of non-zero volume fraction, which leads to the interparticle diffusional interactions, can affect the coarsening of precipitates in two ways: (a) Different from the LSW theory claiming that the coarsening rate of a precipitate is solely a function of the precipitate size, the coarsening rate in the modified theories takes the surrounding precipitates into consideration. The precipitate will coarsen when surrounded by precipitates smaller than it and shrink when the surrounding precipitates are larger. (b) In the case where LSW theory is applied, the spatial distribution is considered as random. While in modified theory where the diffusional interaction is included, it is unlikely to find a small and large precipitate almost touching, as the small precipitate tends to shrink when it is close to a larger precipitate. Thus, the average interparticle separation is larger than that in a system with a random spatial distribution. Nevertheless, large precipitates prefer to locate near small precipitates as the small ones can feed solute for their coarsening. Therefore, both precipitate size and spatial distribution have to be considered when discussing the effect of volume fraction on the coarsening behaviours.

According to the modified theories, the coarsening rate constants $K_{R}$ increase with the volume fraction $f_{p}$, and the time-invariant PSDs become broader and more 
symmetric as the precipitated volume fraction increases. However, Brailsford and Wynblatt [136] suggested that the coarsening rate constants are much less sensitive to the volume fraction and thus are more compatible with experimental observation. For the evolution of PSDs, the situation is complicated. One possible reason for the broadening is that the continuous nucleation during the precipitation provides an increase in the population of fine precipitates. Secondly, the coarsening of large precipitates at the expense of fine ones results in the increase of the number of large precipitates.

In terms of this work, experimental observations on the coarsening behaviour of precipitates in Mn-based maraging steels revealed that the evolution of PSDs with the increasing volume fraction coincides with the prediction by modified LSW theories, which indicates the effect of precipitate volume fraction cannot be ignored. In the case of coarsening kinetics, the experimental evidence reveal that at the early aging stage (Figure 5-4), precipitates exhibited an interface-dominated growth followed by a decrease in the growth rate indicating the initiation of the diffusiondominated coarsening. The coarsening rate constant $K_{R}$ obeys the diffusioncontrolled coarsening kinetics predicted by LSW theory (Figure 5-5). It is also revealed that the Mn content of the alloy, which is not the main precipitate forming element, has a significant effect on the growth and coarsening kinetics of precipitates. This will be discussed in detail in Section 5.8.3.

\subsubsection{The formation mechanism of core/shell structure}

The most well-known precipitate with core/shell structure is, $\mathrm{Al}_{3}(\mathrm{Sc}, \mathrm{Zr})$ precipitates in Al-Zr-Sc alloys which have been widely investigated based on both experimental analyses and complementary first-principle calculations [229]. Elagin et al. first claimed that $\mathrm{Zr}$ could substitute the $\mathrm{Sc}$ atoms in $\mathrm{Al}_{3} \mathrm{Sc}$ precipitates in an $\mathrm{Al}-0.24 \mathrm{Sc}-$ 0.04Zr alloy [230]. Later, HREM studies [229][231][232] revealed that $\mathrm{Zr}$ was detected at the $\alpha-\mathrm{Al} / \mathrm{Al}_{3}\left(\mathrm{Sc}_{1-\mathrm{x}} \mathrm{Zr}_{\mathrm{x}}\right)$ interfaces. Then APT analyses further quantified an 8-10 at.\% $\mathrm{Zr}$ concentration at the interface [233]. It is suggested that $\mathrm{Zr}$ acts as a barrier to the diffusion of Sc, and contributes to lowering the coarsening rate of 
precipitates [234]. These experimental observations agree with the prediction by the thermodynamic calculation and model simulations [235].

Another well-known group is Cu-bearing precipitation-strengthening Fe alloys. In $\mathrm{Fe}-\mathrm{Cr}-\mathrm{Ni}-\mathrm{Al}-\mathrm{Cu}$ alloys [38][39], the decomposition of the $\mathrm{Ni}+\mathrm{Al}+\mathrm{Cu}$ clusters leads to a core/shell precipitates with $\mathrm{Cu}$-rich core surrounded by a NiAl shell. The thermally stable $\mathrm{NiAl}$ shells hinder the diffusion growth of $\mathrm{Cu}$ core and more importantly, reduce the misfit between the fcc $\mathrm{Cu}$-rich core and bcc matrix. While in the conventional maraging steels strengthened by $\mathrm{Ni}_{3} \mathrm{Ti}$ precipitates, the formation mechanism of core/shell structure is explained as: since the solubility of $\mathrm{Cu}$ in $\mathrm{Ni}_{3} \mathrm{Ti}$ phase is low, the $\mathrm{Cu}$-rich clusters usually act as nucleation sites for $\mathrm{Ni}_{3} \mathrm{Ti}$ precipitates and thus a unique core/shell precipitate with $\mathrm{Cu}$ embedded in $\mathrm{Ni}_{3} \mathrm{Ti}$ phase can be found in these maraging steels. Detailed discussion about the core/shell structure in $\mathrm{Cu}$-containing $\mathrm{Fe}$ alloys has been given in Section 2.2.3.5.

Both thermodynamic and kinetic factors were studied in order to understand the formation of the core/shell structure in precipitates. Table 5-3 presents the diffusion rates of the constituent elements in bcc-Fe at $500{ }^{\circ} \mathrm{C}$. Al possesses the highest diffusion rate followed by $\mathrm{Ti}$ and $\mathrm{Mn}$ which have the same order of magnitude. $\mathrm{Ni}$, together with $\mathrm{Fe}$ itself, has the slowest diffusion rates in bcc-Fe at $500{ }^{\circ} \mathrm{C}$. Except that $\mathrm{Al}$ has the highest diffusion rate which is about five or six orders of magnitude faster than other elements, the difference of the diffusion rates among Ti, Mn, Ni and Fe are not large. The APT analyses on a $9 \% \mathrm{Mn}$ maraging steel reported that at the very early stage of precipitation $\left(0.5 \mathrm{~h}\right.$ at $\left.450{ }^{\circ} \mathrm{C}\right), 50$ at. $\% \mathrm{Fe}, 35$ at. $\% \mathrm{Ni}, 11$ at.\% $\mathrm{Mn}, 2$ at.\% $\mathrm{Al}$ and 2 at.\% $\mathrm{Ti}$ were detected [135]. It is obvious that $\mathrm{Ni}$, which possesses the slowest diffusion rate at $450^{\circ} \mathrm{C}$, had a relatively high concentration in precipitates than other elements (expect the matrix element $\mathrm{Fe}$ ); while $\mathrm{Al}$ and $\mathrm{Ti}$ which possess the fastest diffusion rates, had very low concentrations, thus it is unlikely that the precipitate heterogeneity is associated with the kinetic effect. 
Chapter 5 Characterization of $\mathrm{L2}_{1}$-ordered $\mathrm{Ni}_{2} \mathrm{TiAl}$ intermetallic phase and its precipitation behaviour

Table 5-3 The diffusion rates of the constituent elements in bcc-Fe at $500{ }^{\circ} \mathrm{C}$

\begin{tabular}{cccc}
\hline Element & Diffusion rate $\left(\mathrm{cm}^{2} \mathrm{~s}^{-1}\right)$ & Equation & References \\
\hline $\mathrm{Al}$ & $6.05 \times 10^{-11}$ & $58600 \exp (-53000 / R T)$ & {$[236]$} \\
$\mathrm{Ti}$ & $7.97 \times 10^{-16}$ & $20.01 \exp (-58000 / R T)$ & {$[237]$} \\
$\mathrm{Mn}$ & $5.01 \times 10^{-16}$ & $0.35 \exp (-52500 / R T)$ & {$[238]$} \\
$\mathrm{Ni}$ & $3.54 \times 10^{-17}$ & $1.4 \exp (-58700 / R T)$ & {$[195]$} \\
$\mathrm{Fe}$ & $2.17 \times 10^{-17}$ & $2.0 \exp (-60000 / R T)$ & {$[239]$}
\end{tabular}

It is generally accepted that the precipitation process is governed by complex thermodynamic driving force [109]. The mixing enthalpy of elements, $\Delta H_{\text {mix }}$, are displayed in Table 5-4 to discuss the precipitate stability in Mn-based maraging steels [240]. The three largest negative mixing enthalpy belong to Al-Ti, Ni-Ti and $\mathrm{Al}-\mathrm{Ni}$, which means there is a high tendency that these three elements will segregate and form a second phase. This conclusion agrees with the experimental results that $\mathrm{Ni}_{2} \mathrm{TiAl}$ is the dominant precipitates in Mn-based maraging steels. In addition, no positive mixing enthalpy in Table 5-4 suggests the other two elements, $\mathrm{Fe}$ and $\mathrm{Mn}$, may also partition to the precipitates, especially considering that both of them have high concentrations in the matrix. In view of the negative mixing enthalpy between elements, the precipitates are not likely to decompose during aging. Therefore, additional factors, such as interfacial energy are taken into consideration. The lattice parameter of $\mathrm{B} 2-\mathrm{NiAl}$ phase $(\mathrm{a}=0.2887 \mathrm{~nm})$ is larger than that of bcc-Fe $(\mathrm{a}=$ $0.2884 \mathrm{~nm})$ but smaller than that of $1 / 8 \mathrm{Ni}_{2} \mathrm{TiAl}$ lattice $(\mathrm{a}=0.2925 \mathrm{~nm})$. Therefore, the formation of $\mathrm{NiAl}$ at the interface is believed to contribute to the further reduction of the lattice misfit between $\mathrm{Ni}_{2} \mathrm{TiAl}$ and bcc-Fe matrix. Previous studies have reported the similar formation of $\mathrm{B} 2$ phase from the parent $\mathrm{L} 2{ }_{1}$ phase in a $\mathrm{Ni}$ Ti-Al alloy [86]. Besides, a two phase $\mathrm{L}_{2}{ }_{1}-\mathrm{Ni}_{2} \mathrm{TiAl} / \mathrm{B} 2-\mathrm{NiAl}$ precipitates formed by spinodal decomposition: $L 2_{1} \rightarrow L 2_{1}+B 2$ were also observed in a ferritic alloy [85]. 
Chapter 5 Characterization of $\mathrm{L}_{1}$-ordered $\mathrm{Ni}_{2} \mathrm{TiAl}$ intermetallic phase and its precipitation behaviour

It is proposed that the $\mathrm{L}_{1}{ }_{1}-\mathrm{Ni}_{2} \mathrm{TiAl} / \mathrm{B} 2-\mathrm{NiAl}$ precipitate is a more thermodynamically stable structure in the Mn-based maraging steels.

Table 5-4 The mixing enthalpy of elements in the Mn-based maraging steels [240].

\begin{tabular}{cccccc}
\hline$\Delta H_{\text {mix }}\left(\mathrm{kJmol}^{-1}\right)$ & $\mathrm{Al}$ & $\mathrm{Ti}$ & $\mathrm{Mn}$ & $\mathrm{Fe}$ & $\mathrm{Ni}$ \\
\hline $\mathrm{Al}$ & - & -30 & -19 & -11 & -22 \\
$\mathrm{Ti}$ & - & - & -8 & -17 & -35 \\
$\mathrm{Mn}$ & - & - & - & 0 & -8 \\
$\mathrm{Fe}$ & - & - & - & - & -2 \\
$\mathrm{Ni}$ & - & - & - & - & - \\
\hline
\end{tabular}

\subsubsection{The effect of Mn on the precipitation}

The microstructural observation revealed that the nucleation of $\mathrm{Ni}_{2} \mathrm{TiAl}$ was promoted by the Mn content of the alloy. As shown in Figure 5-1, a higher number density of precipitates was observed in the $12 \% \mathrm{Mn}$ alloy compared to the $7 \% \mathrm{Mn}$ alloy for the same aging conditions. Osamura et al. [241] found that $\mathrm{Ni}$ and $\mathrm{Mn}$ additions promoted the precipitation reaction in a $\mathrm{Fe}-\mathrm{Cu}$ alloy. Jiao et al. [106] also reported that the additions of $\mathrm{Ni}$ and $\mathrm{Mn}$ in $\mathrm{Fe}-\mathrm{Cu}$ alloy were beneficial to increasing the number density of precipitates. Complementary Langer-Schwartz simulation and first-principles calculation further demonstrated that Ni had a more significant effect than $\mathrm{Mn}$ on decreasing the critical energy for nucleation [242][243].

According to the classical nucleation theory [244], the nucleation of $\mathrm{Ni}_{2} \mathrm{TiAl}$ precipitates can be described by:

$$
\frac{d N}{d t} \propto\left(\frac{-\sigma_{n}}{k T}\right)
$$

where $\sigma_{n}$ is the critical energy for nucleation, $k$ is the Boltzmann's constant and $T$ is the temperature in kelvin. The critical energy for nucleation is expressed as: 


$$
\sigma_{n}=\frac{16 \pi \sigma_{\alpha / \beta}^{3}}{3\left(\sigma_{V}+\sigma_{e l}\right)^{2}}
$$

where $\sigma_{\alpha / \beta}$ is the interfacial free energy per unit area between the precipitate and matrix, $\Delta G_{V}$ is the chemical driving force of precipitates per unit volume and $\sigma_{e l}$ is the elastic strain energy. Therefore, the effect of Mn content on reducing $\sigma_{n}$ can be discussed in two aspects: the elastic strain energy for nucleation and the precipitate/matrix interfacial energy. APT analyses on the early precipitation stage illustrated that the precipitates in a $9 \%$ Mn-based maraging steel contained a considerable Mn content (11 at. $\% \mathrm{Mn}$ at $450{ }^{\circ} \mathrm{C} / 0.5 \mathrm{~h}$ ) [135], which indicates that Mn was involved in the nucleation of precipitates. The study by Zhang et al. revealed that $\mathrm{Mn}$ has the capability of reducing the misfit strain energy at interface, namely, when $\mathrm{Mn}$ atoms enter the nuclei, the lattice parameter of the nuclei can be decreased and the strain energy barrier for nucleation is also decreased [242]. On the other hand, as the diffusivity of the constituent elements in Table 5-3 implies, Mn has a relatively fast diffusion rate. Moreover, the high mixing enthalpy of Fe/Mn (Table 54), indicates that $\mathrm{Mn}$ tends to segregate from the Fe matrix. Based on these two reasons, it is highly possible that these Mn-rich clusters acts as nucleation sites for further precipitation, and thus the number density of precipitate and its growth rate was largely dependent on the Mn content.

\subsection{Summary}

1. Conventional TEM-EDS and STEM-EDS analyses demonstrate that the nano-scale precipitates in Mn-based maraging steels is a non-stoichiometric $\mathrm{Ni}_{2}$ TiAl phase with a small amount of $\mathrm{Fe}$ and $\mathrm{Mn}$ substitution. This nonstoichiometric composition is believed to enhance the ductility of the phase [103].

2. HREM combined with SAED study identifies the crystal structure of the precipitate. The $\mathrm{L} 2{ }_{1}$-ordered $\mathrm{Ni}_{2} \mathrm{TiAl}$ phase is expected to have a better creep resistance than $\mathrm{B} 2-\mathrm{NiAl}$ phase [91]. The orientation relationship between the L2 ${ }_{1}$-ordered $\mathrm{Ni}_{2} \mathrm{TiAl}$ phase and bcc martensite matrix is found to be $(220)_{L 2_{1}}\left\|(110)_{b c c} \quad, \quad[001]_{L 2_{1}}\right\|[001]_{b c c} ; \quad(220)_{L 2_{1}} \|(110)_{b c c}$, 
$[011]_{L 2_{1}}\left\|[011]_{b c c} ;(220)_{L 2_{1}}\right\|(110)_{b c c},[\overline{1} 11]_{L 2_{1}} \|[\overline{1} 11]_{b c c}$. One eighth of the crystal structure of $\mathrm{L} 2{ }_{1}-\mathrm{Ni}_{2} \mathrm{TiAl}$ phase is a bcc cubic lattice which has a similar lattice parameter with the bcc matrix. The lattice misfit between them is calculated as $1.24 \%$. This small lattice misfit together with the special orientation relationship illustrates that the $\mathrm{L}_{1}{ }_{1}$-ordered $\mathrm{Ni}_{2} \mathrm{TiAl}$ precipitates is not only coherent but also coplanar with the matrix. The dumbbell-shaped precipitates in the 10080 min aged sample demonstrate that the precipitates maintained the coherency with the matrix even after long-term aging treatment (Figure 5-14).

3. The shell of the precipitates in Mn-based maraging steels is found to have a strong resistance to coarsening and maintain a thickness of 2-3 $\mathrm{nm}$ regardless of the growth of core during aging. The presence of core/shell structure indicates the heterogeneity in precipitates. A sharp step of contrast at the periphery under HAADF observation (Figure 5-16) excludes the possibility of thickness contrast (assuming the precipitates are spherical) and thus confirms the sharp contrast corresponds to the mass difference. HREM analyses at the periphery suggest that the lattice structure of the shell is uniform with that of the core despite a slight distortion at interface. Along with the evidence of HAADF and dark-field micrograph, it is proposed that the core is $\mathrm{Ni}_{2} \mathrm{TiAl}$ and the shell is $\mathrm{NiAl}$. It is believed that the $\mathrm{L} 2_{1}$ $\mathrm{Ni}_{2}$ TiAl/B2-NiAl structure can further decrease the lattice misfit between the precipitate and bcc-Fe matrix.

4. Owing to the non-zero volume fraction in reality, a broadening of the experimental PSDs than the theoretical PSD function is evident, especially at the longest aging time when the volume fraction of precipitates cannot be ignored. However, the discrepancy is not that obvious in the coarsening rate as the coarsening rate constants $\left(K_{R}\right)$ are much less sensitive to the volume fraction compared to the PSDs [136]. Experimental results reveal that coarsening rate constants of precipitates in the three alloys follow the diffusion-controlled coarsening kinetic form $\bar{r}^{3} \sim K t$ predicted by LSW theory. 
5. Microstructural observation reveals a higher number density and larger size of precipitates in $12 \% \mathrm{Mn}$ alloy compared to $7 \%$ and $10 \% \mathrm{Mn}$ alloys for the same aging condition. Both thermodynamic and kinetic analyses suggest that Mn atoms participated in the nucleation of precipitates and reduced the misfit strain energy at the interface [106]. Therefore, higher Mn content is believed to promote the nucleation and thus the growth and coarsening of precipitates. The faster coarsening rate as well as the higher volume fraction of precipitate in $12 \% \mathrm{Mn}$ alloy is correlated to the large number of nucleation sites due to Mn segregation. 


\section{Chapter 6 The formation mechanism of reverted austenite and its mechanical stability}

\subsection{Introduction}

As most Fe-Mn-(Ni) maraging alloys exhibit a poor ductility due to the $\mathrm{Mn}$ segregation at grain boundaries, many studies have been carried out to improve their ductility and some of them proposed that the ductility can be improved by generating reverted austenite via aging at higher temperatures or for longer times.

In terms of the Mn TRIP steels, in the 1960s Goldshtein et al. [12] first reported an excellent toughness in an $8 \% \mathrm{Mn}$ steel with a considerable amount of austenite. Later Miller found that, by intercritical annealing, 20-40\% austenite was obtained in 0.1-5 wt.\% Mn steels with excellent stability [24]. Based on Miller's work, Luo et al. modified the composition of $5 \mathrm{wt} \% \mathrm{Mn}$ steels and $30 \%$ austenite was formed in the new alloy, and thereby, the tensile strength was improved to 1-1.5 GPa and total elongation to $31-44 \%$ [27].

In this study, the approach to form Mn-rich austenite is different from the above intercritical annealing process in Mn TIRP steels. The austenite reversion in Mnbased maraging steels took place simultaneously with precipitation when aging at relatively high temperature. The difference in the heat treatment process led to the microstructure and mechanical properties of reverted austenite being different from those of retained austenite. It is worth emphasising that as the austenite reversion occurred simultaneously with the precipitation, with $\mathrm{Mn}$ atoms involved in both processes, and therefore there might expect to be a competition in the role of $\mathrm{Mn}$.

As discussed in Section 2.4.1, both the amount and stability of austenite are of great importance for the mechanical properties of steels containing austenite, based on which the optimization of microstructure/properties can be feasible. Therefore, the major aim of this chapter is to characterizing the austenite phase in different aging conditions and investigating its formation mechanisms. The influence of the quantity 
of reverted austenite on the mechanical properties will be discussed in the next chapter.

\subsection{SEM observation}

\subsubsection{The effect of aging parameters on austenite reversion}

The typical microstructure of reverted austenite in $\alpha^{\prime}$-martensite matrix along with uniformly distributed precipitates has been given in Figure 4-7. In this section, the representative $12 \% \mathrm{Mn}$ alloy was studied to explore the effects of aging temperatures/times on austenite reversion.

Aging at $420{ }^{\circ} \mathrm{C}$ even for 10080 minutes did not result in any detectable austenite phase, however, a very small amount of matrix austenite layers at the prior austenite grain boundaries at the martensite packet boundaries were observed (Figure 6-1(a)). In contrast to the sluggish austenite reversion, a large number density of nano-scaled precipitates has been formed at this stage (Figure 6-1(b)).

At $460{ }^{\circ} \mathrm{C}$, the sample after aged for 2880 minutes also exhibited a typical microstructure of the austenite layers along boundaries (Figure 6-1(c)). The matrix austenite at prior austenite boundaries, denoted ' $M$ ', appears thinner and smoother than austenite layers at martensite lath/packet boundaries. As expected, prolonged aging for 10080 minutes led to the austenite layers growing into lath-like austenite, as shown in Figure 6-1(d). 

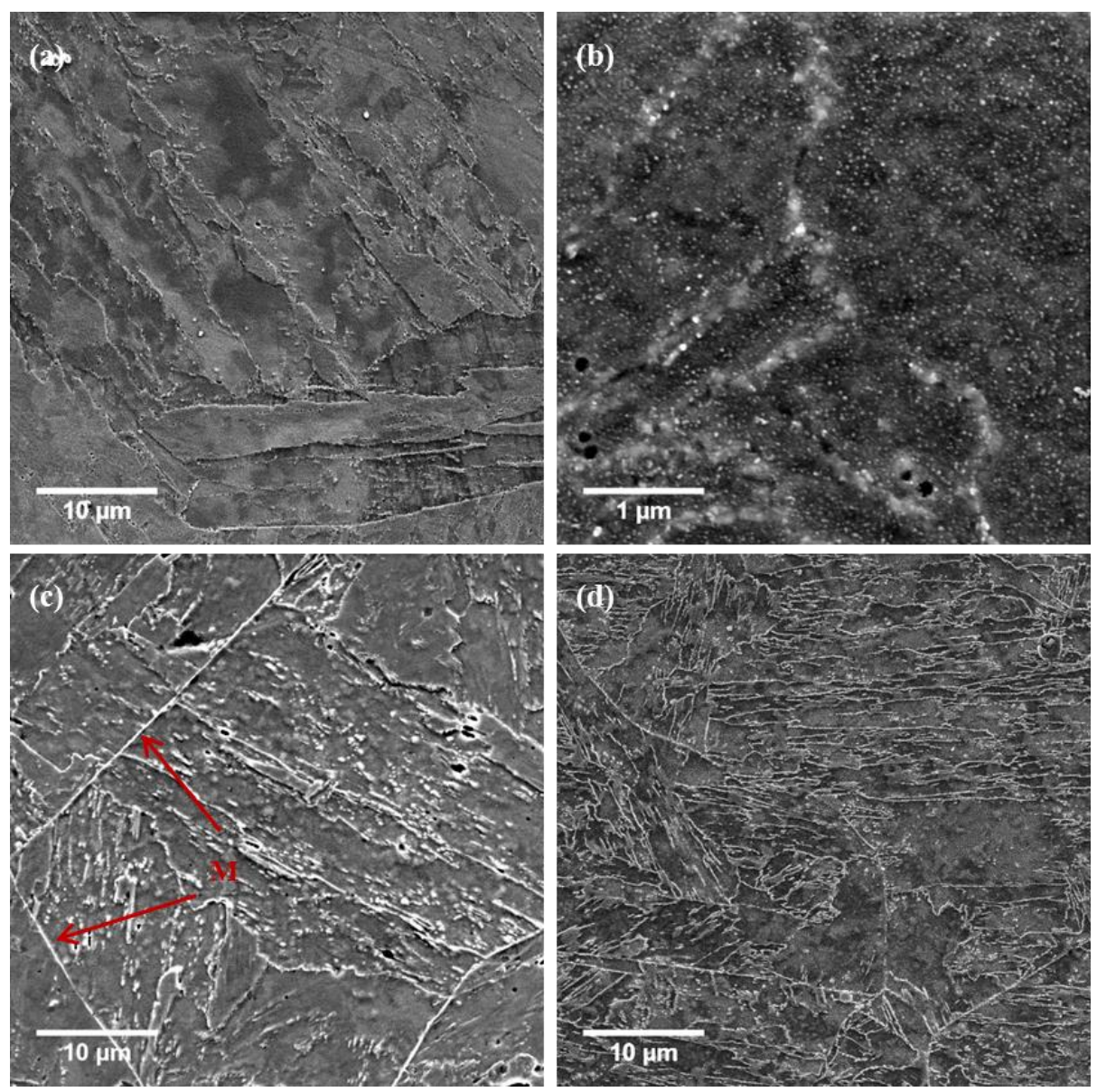

Figure 6-1 SEM micrographs showing the morphologies of reverted austenite in the $12 \% \mathrm{Mn}$

alloy during aging. (a) Thin austenite layers formed along boundaries and (b) highly dispersed fine precipitates at $420{ }^{\circ} \mathrm{C} / 10080 \mathrm{~min}$ state; (c) evident matrix austenite and austenite layers at $460{ }^{\circ} \mathrm{C} / 2880 \mathrm{~min}$ state; (d) the amount of austenite increased after aging at $460{ }^{\circ} \mathrm{C}$ for $10080 \mathrm{~min}$.

When increasing the aging temperature to $500{ }^{\circ} \mathrm{C}$, ultrafine precipitates were generated at the onset of aging, as shown in Figure 6-2(a). Thin matrix austenite and austenite layers at boundaries were also visible in the $10 \mathrm{~min}$ aged state (Figure 6-2(b)). After a longer aging time, the austenite layers gradually grew into film-like and then lath-like austenite (Figure 6-2(c) and (d)). The lath-like austenite was formed not only along the boundaries, but also within the martensite laths. It is found that the lath-like austenite and residual martensite present a lamellar structure (see inset in Figure 6-2(d)). 

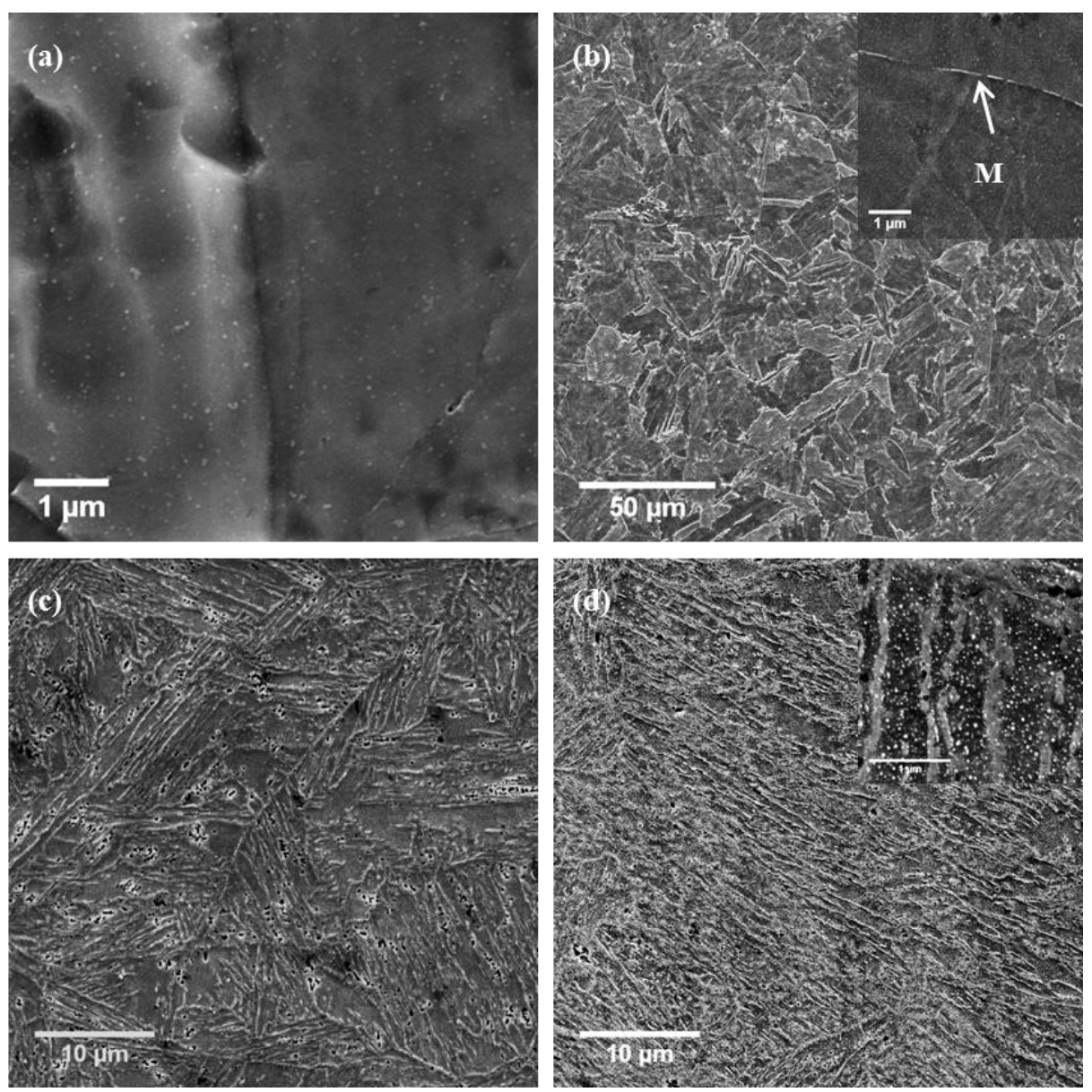

Figure 6-2 (a) Fine precipitates and (b) thin matrix austenite at the onset of aging in the $12 \%$ Mn alloy $\left(500{ }^{\circ} \mathrm{C} / 10 \mathrm{~min}\right.$ state); (c) the lath-like austenite became dominant after aging for $2880 \mathrm{~min}$; (d) a lamellar structure of alternative lath-like austenite and residual martensite in the 10080 min aged sample.

Raising the aging temperature to $540{ }^{\circ} \mathrm{C}$ led to a further increase in the driving force for austenite reversion. The initial point for austenite reversion was shorter than 10 minutes, so it was not captured. The amounts of reverted austenite formed at $540{ }^{\circ} \mathrm{C}$ were higher than that formed at $500{ }^{\circ} \mathrm{C}$ for the same durations (Figure 6-3(a)). In addition, the thickness of lath-like austenite was also larger (Figure 6-3(b)). 

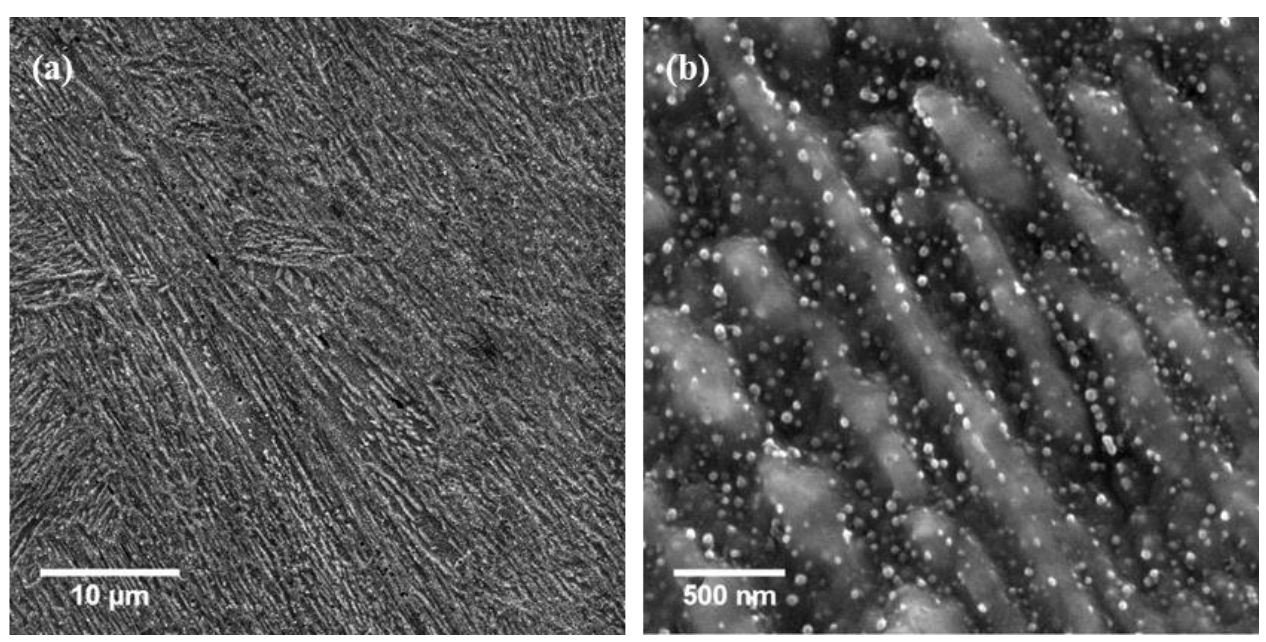

Figure 6-3 (a) Reverted austenite in the $12 \% \mathrm{Mn}$ alloy after aging at $540{ }^{\circ} \mathrm{C}$ for $10080 \mathrm{~min}$; (b) reverted austenite is free of precipitates.

\subsubsection{The effect of Mn content on austenite reversion}

The kinetics of austenite reversion in the $7 \% \mathrm{Mn}$ alloy was very slow. No reverted austenite was present within $2880 \mathrm{~min}$ when aging at $500{ }^{\circ} \mathrm{C}$, but nano-precipitates have been found uniformly distributed in $\alpha^{\prime}$-martensite matrix (Figure 6-4(a)). Even after aging for $10080 \mathrm{~min}$ did not lead to an evident increase of the amount of reverted austenite, only matrix austenite layers was observed (Figure 6-4(b)). In the $10 \% \mathrm{Mn}$ alloy, the amount of reverted austenite was much higher than that in $7 \% \mathrm{Mn}$ alloy for the same aging conditions. It also appears that the aging time did not appreciably affect the quantity of reverted austenite in the late aging stage, with the amount of reverted austenite in the 10080 min aged sample (Figure 6-4(d)) not much higher than that in the 2880 min aged sample (Figure 6-4(c)). In addition, the amount of reverted austenite in the $12 \% \mathrm{Mn}$ alloy (Figure 6-2) was also much higher than that in 10\% Mn alloy (Figure 6-4) for the same aging conditions. Clearly, therefore, the higher the Mn content of the alloy, the greater the austenite reversion. 

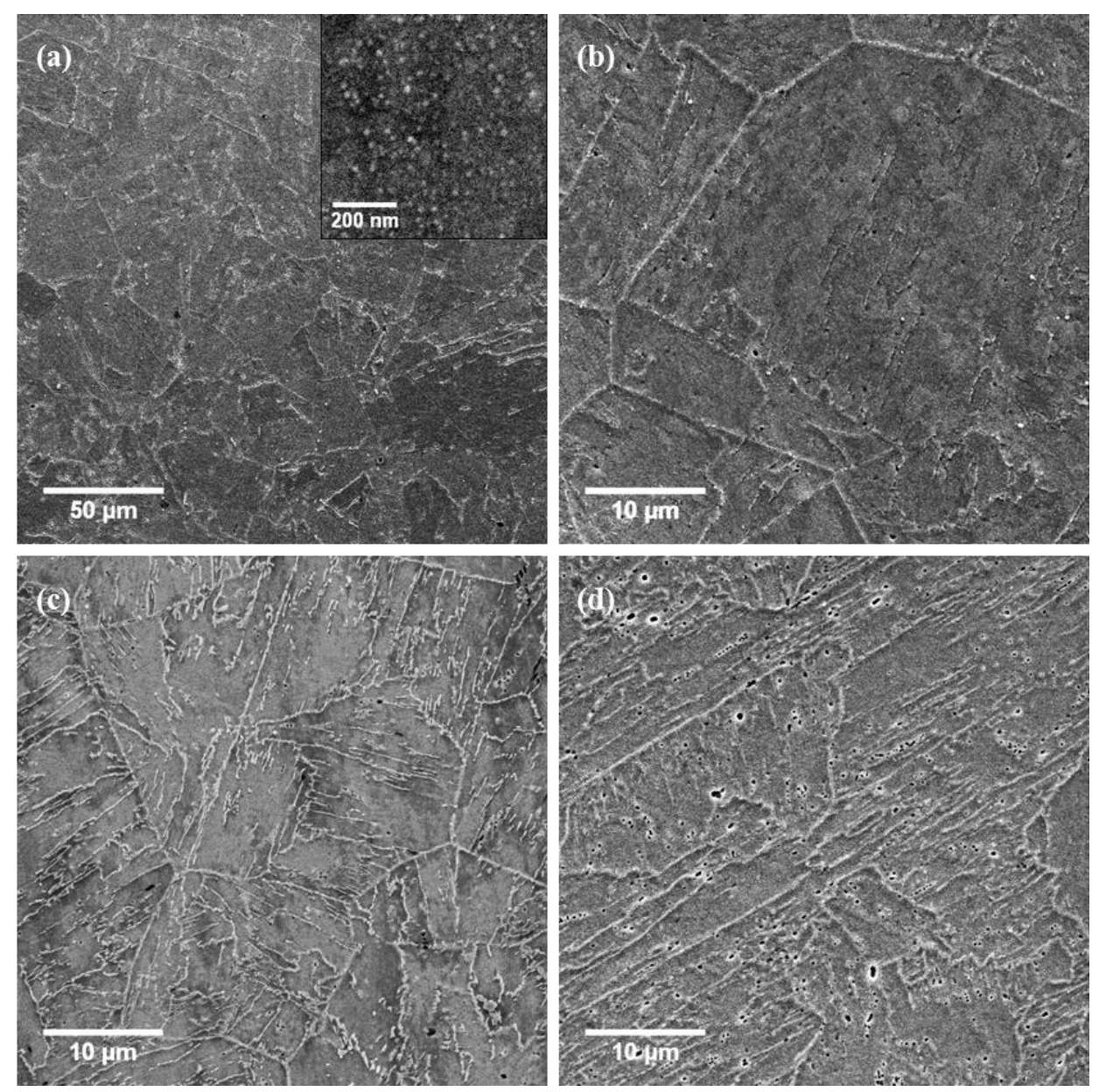

Figure 6-4 SEM observation of (a) 7\% Mn alloy (500 ${ }^{\circ} \mathrm{C} / 2880 \mathrm{~min}$ state); (b) $7 \% \mathrm{Mn}$ alloy $\left(500{ }^{\circ} \mathrm{C} / 10080 \mathrm{~min}\right.$ state); (c) $10 \% \mathrm{Mn}$ alloy $\left(500{ }^{\circ} \mathrm{C} / 2880 \mathrm{~min}\right.$ state); (d) $10 \% \mathrm{Mn}$ alloy $\left(500{ }^{\circ} \mathrm{C} / 10080 \mathrm{~min}\right.$ state).

\subsection{TEM investigation}

TEM investigations were conducted on samples in which reverted austenite could be observed. At the beginning of austenite reversion (e.g. $10 \% \mathrm{Mn}$ alloy, $500{ }^{\circ} \mathrm{C} / 1440$ min state (Figure 6-5(a)) or when the kinetics of austenite reversion was very slow, e.g. $7 \% \mathrm{Mn}$ alloy, $500{ }^{\circ} \mathrm{C} / 10080 \mathrm{~min}$ state (Figure 6-5(b))), only thin matrix austenite, which looked like nanolayer, could be found along the prior austenite grain boundaries and this was consistent with what had been observed under SEM observations. 

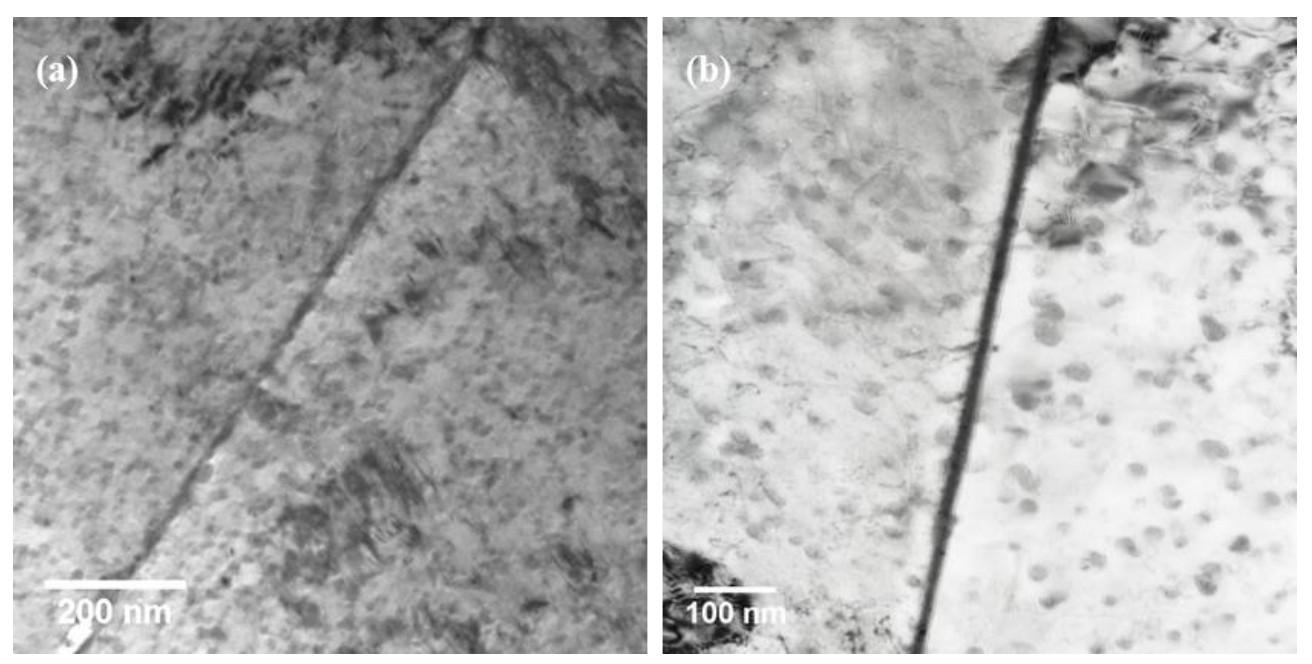

Figure 6-5 Matrix austenite formed along prior austenite grain boundaries in (a) $10 \% \mathrm{Mn}$ alloy at $500{ }^{\circ} \mathrm{C} / 1440 \mathrm{~min}$ state and (b) $7 \% \mathrm{Mn}$ alloy at $500{ }^{\circ} \mathrm{C} / 10080 \mathrm{~min}$ state.

With the growth of austenite, the dominant morphology of reverted austenite gradually became film-like and then lath-like. Images of lath-like austenite from different orientations parallel to the elongated austenite were shown in Figure 6-6. Besides, the TEM observation demonstrated that the reverted austenite was free of precipitates, whereas a high dislocation density induced from cooling was observed within the austenite grains. 

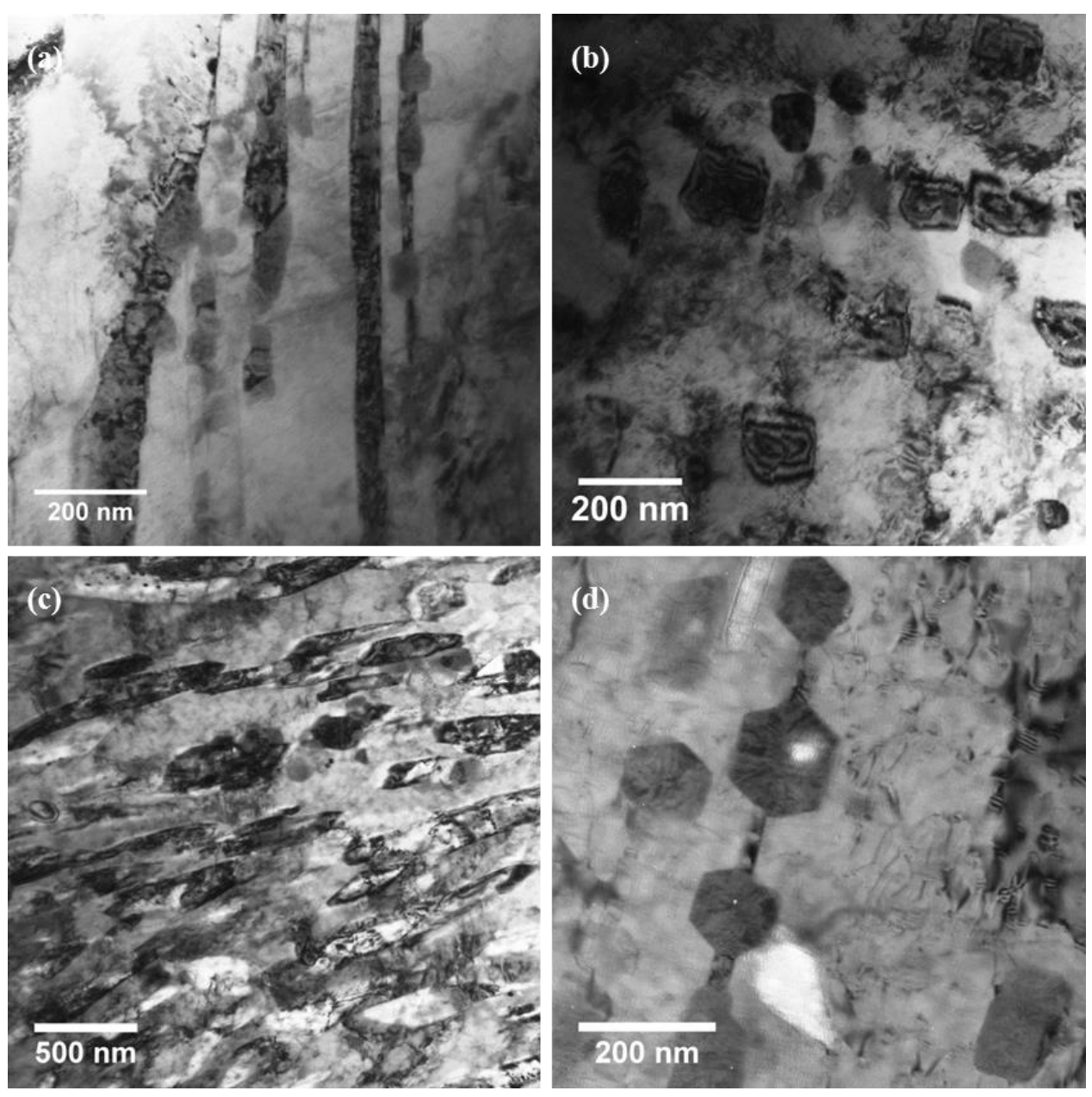

Figure 6-6 TEM micrographs of lath-like austenite in (a) $12 \% \mathrm{Mn}$ alloy aging at $500{ }^{\circ} \mathrm{C}$ for $2880 \mathrm{~min}$ and (b) $12 \% \mathrm{Mn}$ alloy aging at $540{ }^{\circ} \mathrm{C}$ for $2880 \mathrm{~min}$; (c) and (d) are the corresponding images from a different orientation.

The evolution of the chemical composition in reverted austenite and adjacent martensite was analysed by TEM-EDS. The EDS analyses of the three alloys are presented in Table 6-1 and Table 6-2. Each data is the average of at least 5 measurements.

It is worth noting that the $\mathrm{Al}$ contents of solution-treated matrix in the three alloys were higher than the nominal content of the alloys (Al: $\sim 2.5$ at.\%). This is probably due to the inaccuracy of the semi-quantitative analysis using EDS in the TEM, where $\mathrm{Al}$ gives the second highest peak per atomic fraction for the whole periodic table. Otherwise, the detected compositions of the martensite matrix in the SHT state are consistent with the nominal compositions of alloys. Schnitzer et al. [147] who 
compared the composition results measured by TEM-EDS and APT when studying the reverted austenite in a PH 13-8 maraging steels, also stated that there was no evident difference between the results obtained by the two methods, except that the Al concentration by the TEM-EDS measurement was slightly higher than that obtained by the APT measurement.

Table 6-1 presents the chemical compositions of austenite and martensite in the 7\% Mn alloy. As the kinetics of austenite reversion in the 7\% $\mathrm{Mn}$ alloy was slow, the composition analysis was only performed on film-like austenite (nanolayer) formed after aging at $500{ }^{\circ} \mathrm{C}$ for a long time. The Mn concentration of austenite nanolayer increased with aging time, from $12.9 \pm 0.6$ at.\% in the $500{ }^{\circ} \mathrm{C} / 5760 \mathrm{~min}$ state to $16.2 \pm 0.3$ at.\% in the $500{ }^{\circ} \mathrm{C} / 10080 \mathrm{~min}$ state. What was unexpected is that the austenite nanolayer was also enriched in $\mathrm{Ti}$ and Mo. The Ti concentration of the austenite maintained at a level of 12.4-14.5 at.\% and the Mo concentration maintained between 10.5 at.\% and 13.6 at.\%. A similar Ti and Mo enrichment in austenite was also observed in the $10 \% \mathrm{Mn}$ alloy in the early stage of austenite reversion. This finding is very interesting as $\mathrm{Ti}$ is generally considered to raise the $A_{e}^{3}$ temperature and therefore is not expected in the austenite phase. However, $\mathrm{Ni}$, as an austenite stabilizer element, was present at a low concentration of 1.2-1.9 at.\% in the reverted austenite. On the other hand, the composition of martensite matrix was approximately constant during the aging process, for example, the composition of matrix in the $500{ }^{\circ} \mathrm{C} / 10080$ min state was similar to that in the SHT state (Table 61). 


\section{Chapter 6 The formation mechanism of reverted austenite and its mechanical stability}

Table 6-1 Chemical compositions (at.\%) of reverted austenite and martensite in the 7\% $\mathrm{Mn}$ alloy obtained by TEM-EDS

\begin{tabular}{|c|c|c|c|c|}
\hline \multirow{3}{*}{ Elements } & \multirow{3}{*}{$\begin{array}{l}\text { SHT } \\
\text { Matrix }\end{array}$} & \multicolumn{3}{|c|}{$500^{\circ} \mathrm{C}$} \\
\hline & & \multirow{2}{*}{$\begin{array}{l}5760 \text { min } \\
\text { Austenite }\end{array}$} & \multicolumn{2}{|c|}{$10080 \mathrm{~min}$} \\
\hline & & & Matrix & Austenite \\
\hline $\mathrm{Al}$ & $5.0 \pm 0.6$ & $2.7 \pm 0.5$ & $3.7 \pm 0.6$ & $1.8 \pm 0.4$ \\
\hline Mo & $1.4 \pm 0.3$ & $13.6 \pm 1.0$ & $1.3 \pm 0.1$ & $10.5 \pm 0.3$ \\
\hline $\mathrm{Ti}$ & $1.1 \pm 0.1$ & $14.5 \pm 0.8$ & $1.1 \pm 0.3$ & $12.4 \pm 0.5$ \\
\hline $\mathrm{Mn}$ & $6.3 \pm 0.3$ & $12.9 \pm 0.6$ & $6.4 \pm 0.2$ & $16.2 \pm 0.3$ \\
\hline $\mathrm{Fe}$ & $84.4 \pm 0.6$ & $54.4 \pm 1.5$ & $85.3 \pm 1.5$ & $58.0 \pm 0.6$ \\
\hline $\mathrm{Ni}$ & $1.8 \pm 0.1$ & $1.9 \pm 0.4$ & $1.9 \pm 0.6$ & $1.2 \pm 0.4$ \\
\hline
\end{tabular}

The chemical compositions of lath-like reverted austenite in the $12 \% \mathrm{Mn}$ alloy are listed in Table 6-2. The lath-like reverted austenite formed in the 12\% Mn alloy had a considerably higher Mn concentration (24-26.1 at.\%) compared to that in the $7 \%$ Mn alloy. While the Ti and Mo concentrations in the reverted austenite (Mo: 2.9-7.3 at.\%, Ti: 2.9-8.0 at.\%) were lower than those in the 7\% Mn alloy. On the other hand, there was an inversed relationship between the Mn concentration of the matrix and the aging time (the Mn concentration reduced from 11.5 at.\% in the SHT state to 6.9 at. $\%$ after aging at $500{ }^{\circ} \mathrm{C}$ for a long time), which is reasonable as the formation of reverted austenite consumed a large amount of $\mathrm{Mn}$ from the martensite matrix. 
Table 6-2 Chemical compositions (at.\%) of reverted austenite and martensite in the 12\% Mn alloy under various heat treatment conditions obtained by TEM-EDS

\begin{tabular}{|c|c|c|c|c|c|c|c|c|}
\hline \multirow{3}{*}{ Elements } & \multirow{3}{*}{$\begin{array}{c}\text { SHT } \\
\text { Matrix }\end{array}$} & \multirow{2}{*}{\multicolumn{2}{|c|}{$\begin{array}{c}460{ }^{\circ} \mathrm{C} \\
10080 \mathrm{~min}\end{array}$}} & \multicolumn{4}{|c|}{$500^{\circ} \mathrm{C}$} & \multirow{3}{*}{$\begin{array}{c}540{ }^{\circ} \mathrm{C} \\
2880 \mathrm{~min} \\
\text { Austenite }\end{array}$} \\
\hline & & & & \multicolumn{2}{|c|}{$2880 \mathrm{~min}$} & \multicolumn{2}{|c|}{$10080 \mathrm{~min}$} & \\
\hline & & Matrix & Austenite & Matrix & Austenite & Matrix & Austenite & \\
\hline $\mathrm{Al}$ & $3.8 \pm 0.8$ & $5.1 \pm 1.4$ & $1.5 \pm 0.2$ & $5.3 \pm 1.0$ & $2.8 \pm 0.4$ & $7.0 \pm 1.5$ & $4.4 \pm 1.0$ & $3.6 \pm 0.7$ \\
\hline Mo & $1.6 \pm 0.2$ & $1.5 \pm 0.4$ & $4.4 \pm 0.5$ & $1.5 \pm 0.6$ & $2.5 \pm 0.6$ & $1.6 \pm 0.5$ & $7.3 \pm 1.6$ & $2.9 \pm 0.4$ \\
\hline $\mathrm{Ti}$ & $1.1 \pm 0.4$ & $1.2 \pm 0.2$ & $3.3 \pm 0.6$ & $1.0 \pm 0.3$ & $2.9 \pm 0.8$ & $1.5 \pm 0.4$ & $8.0 \pm 0.6$ & $5.0 \pm 0.6$ \\
\hline $\mathrm{Mn}$ & $11.5 \pm 0.5$ & $9.8 \pm 0.8$ & $26.1 \pm 1.0$ & $9.6 \pm 0.5$ & $24.1 \pm 1.5$ & $6.9 \pm 0.8$ & $24.7 \pm 1.1$ & $24 \pm 0.8$ \\
\hline $\mathrm{Fe}$ & $80.1 \pm 1.4$ & $80.2 \pm 1.5$ & $63.5 \pm 1.7$ & $81.7 \pm 1.9$ & $66.4 \pm 1.6$ & $80.3 \pm 1.4$ & $53.0 \pm 1.0$ & $63.5 \pm 1.7$ \\
\hline $\mathrm{Ni}$ & $1.9 \pm 0.2$ & $2.2 \pm 0.4$ & $1.2 \pm 0.3$ & $1.7 \pm 0.2$ & $1.3 \pm 0.2$ & $2.6 \pm 0.6$ & $2.5 \pm 0.9$ & $1.0 \pm 0.2$ \\
\hline
\end{tabular}


The SAED analyses reveal that the K-S relationship $\left((\overline{1} 11)_{\gamma} \|(01 \overline{1})_{\alpha}\right.$, $\left.[110]_{\gamma} \|[111]_{\alpha}\right)$ and Nishiyama relationship $\left((\overline{1} 11)_{\gamma}\left\|(0 \overline{1} \overline{1})_{\alpha},[110]_{\gamma}\right\|[100]_{\alpha}\right)$ coexisted between reverted austenite and martensite (Figure 6-7). Some researchers suggested that adjacent austenite grains had nearly the same orientation in low carbon steels and were twin-related [54][171][185]. Due to the limited statistics of the TEM observations, so far there was no evidence from the TEM investigation to prove this. An analysis technique which can provide better statistics, such as electron backscatter diffraction (EBSD), is required for the further study of the orientation relationships between austenite and martensite.
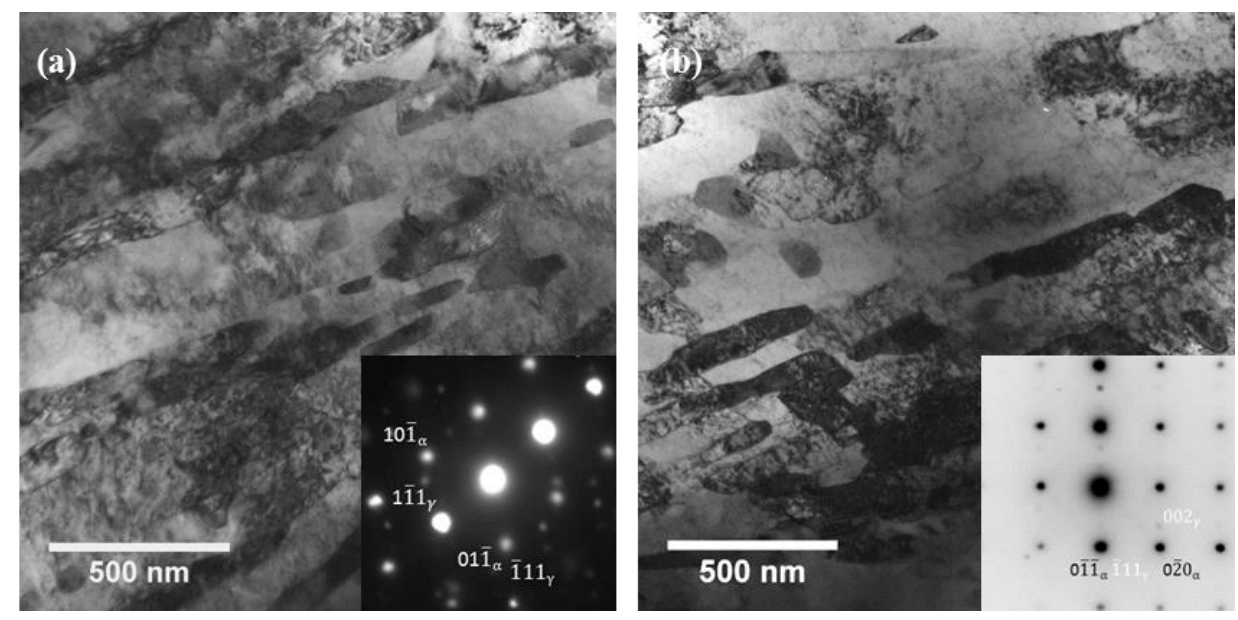

Figure 6-7 Bright-field TEM images of austenite in the martensite matrix and the corresponding selected area diffraction patterns presenting (a) K-S relationship and (b)

Nishiyama relationship between austenite and martensite.

\subsection{XRD quantitative analyses}

As revealed by previous study, Mn-based maraging steels possessed a multi-phase structure comprised of $\alpha^{\prime}$-martensite matrix, $\mathrm{Ni}_{2} \mathrm{TiAl}$ precipitates, reverted austenite and $\varepsilon$-martensite in some conditions. In this section, the volume fractions of reverted austenite formed in different aging conditions were measured by XRD and the results are presented in Table 6-3. In order to better understand the austenite reversion, the evolution of austenite phase fraction in the $10 \%$ and $12 \% \mathrm{Mn}$ alloys aged at $500{ }^{\circ} \mathrm{C}$ is illustrated in Figure 6-8. As shown in Figure 6-8, aging at $500{ }^{\circ} \mathrm{C}$ 
led to a nearly linear increase in the volume fraction of reverted austenite as a function of time up to the maximum was reached for both alloys. In the $10 \% \mathrm{Mn}$ alloy, the austenite phase fraction reached a maximum of $\sim 10 \mathrm{vol} . \%$ in the $500{ }^{\circ} \mathrm{C} /$ 5760 min state after which the content remained approximately constant; while in the $12 \% \mathrm{Mn}$ alloy, the austenite fraction increased linearly up to 25 vol.\% after aging for 4320 min and then slightly decreased with longer aging time.

Table 6-3 The volume fractions (vol.\%) of reverted austenite formed in different aging conditions.

\begin{tabular}{cccccc}
\hline $\begin{array}{c}\text { Aging time } \\
(\min )\end{array}$ & \multicolumn{2}{c}{$10 \% \mathrm{Mn}$} & & \multicolumn{2}{c}{$12 \% \mathrm{Mn}$} \\
\cline { 2 - 3 } \cline { 5 - 6 } \cline { 5 - 6 } & $500{ }^{\circ} \mathrm{C}$ & $460{ }^{\circ} \mathrm{C}$ & & $500{ }^{\circ} \mathrm{C}$ & $460{ }^{\circ} \mathrm{C}$ \\
\hline 1440 & $2.92 \pm 0.67$ & - & & $8.48 \pm 0.73$ & - \\
2880 & $6.03 \pm 0.65$ & - & & $17.13 \pm 1.76$ & - \\
4320 & $7.99 \pm 0.49$ & - & & $24.78 \pm 1.31$ & - \\
5760 & $10.35 \pm 0.73$ & - & & $24.62 \pm 1.49$ & $5.58 \pm 1.24$ \\
10080 & $10.69 \pm 0.45$ & $4.14 \pm 1.03$ & & $23.06 \pm 1.42$ & $9.32 \pm 2.48$ \\
\hline
\end{tabular}

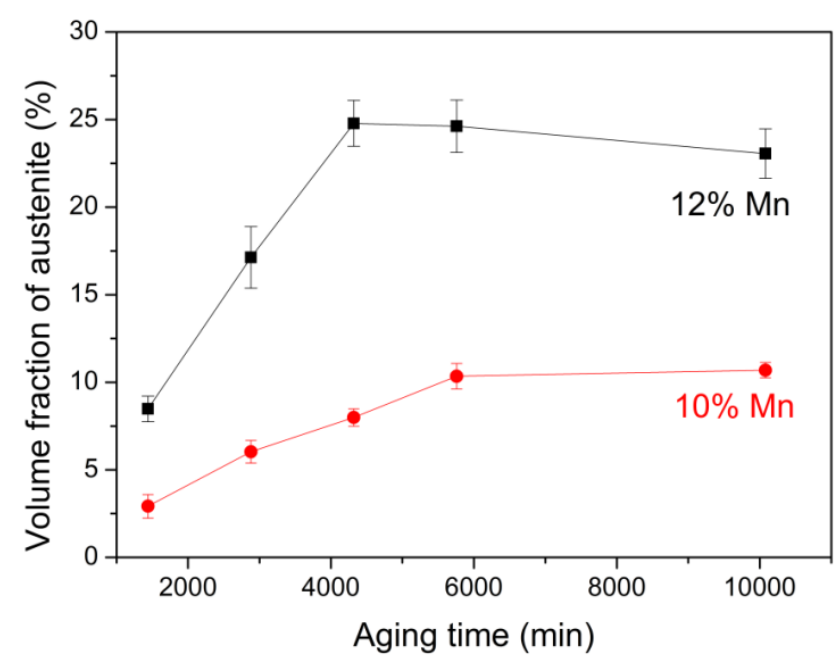

Figure 6-8 The evolution of volume fractions (vol.\%) of reverted austenite formed as a function of aging time at the temperature of $500{ }^{\circ} \mathrm{C}$. 


\subsection{Nanoindentation}

As previously discussed, the key parameters of tensile deformation, i.e. yield strength (YS), ultimate tensile strength (UTS) and total elongation (TE) in multiphase steels, are determined by the fractions of phases and their mechanical stability [140]. Therefore, the contribution of each phase to the strength of the Mn-based maraging steels is the main focus of this study.

In this study, the Mn-based maraging steels in aged conditions were mainly composed of tempered martensite decorated with uniformly dispersed nanoprecipitates and various amount of elongated reverted austenite which depended on the aging conditions and Mn contents. In addition, a trace of $\varepsilon$-martensite or coarse carbide might be present in some cases. The following discussion only considers the martensite (with precipitates embedded within it) and reverted austenite, as previous studies have suggested that these two phases constituted the most relevant microstructure relating to the mechanical properties of materials.

As the mechanical characteristic of reverted austenite (with the size in the range 70$200 \mathrm{~nm}$ ) in this study is hard to obtain by conventional testing methods, the nanoindentation combined with atomic-force microscopy (AFM) was utilized. A cube corner tip, which has a three-sided pyramidal with an apex angle of $90^{\circ}$ and is much sharper than the conventional Berkovich tip, was selected to perform the indentation. In addition, a small load of $70 \mu \mathrm{N}$ was applied. Nanoindentation tests were performed on a number of austenite grains and martensite matrix separately. The typical load-displacement $(P-h)$ curves of the austenite and martensite in the $12 \%$ Mn alloy in the $500{ }^{\circ} \mathrm{C} / 10080$ min state are given in Figure 6-9. Different phases were clearly characterized by different load-displacement relationships. Subject to the maximum load of $70 \mu \mathrm{N}$, the maximum penetration displacement was $10-15 \mathrm{~nm}$ for austenite phase and $\sim 25 \mathrm{~nm}$ for martensite phase. This indicated that in this study the reverted austenite was unexpectedly harder than the martensite. It is generally accepted that the austenite phase, as a fcc crystal, has a low yield strength [245], especially compared to the martensite with highly dispersed nanoprecipitates in maraging steels [52][246][247]. Despite the low yield strength, the working hardening of austenite is remarkable and the higher load required for martensitic 
transformation of austenite phase further increased the strength. In terms of martensite, its high strength is generally due to the high dislocation density and tetragonal lattice containing supersaturated carbon atoms (supersaturated $\mathrm{Mn}, \mathrm{Ni}$, Mo, Ti and Al atoms in Mn-based maraging steels). In this study, as these solidsolution strengthening elements in the martensite matrix were largely depleted by precipitation and austenite reversion and the dislocation density also reduced during the thermal aging process, the martensite could essentially be regarded as ferrite. In addition, a nanoindentation study on a similar medium-Mn TRIP steel revealed that retained austenite was significantly harder than ferrite (austenite: 7.7 GPa, ferrite: 3.4 GPa) [188]. Therefore, it is not surprising to see that the hardness of tempered martensite after a long-term aging was lower than that of austenite in this study.

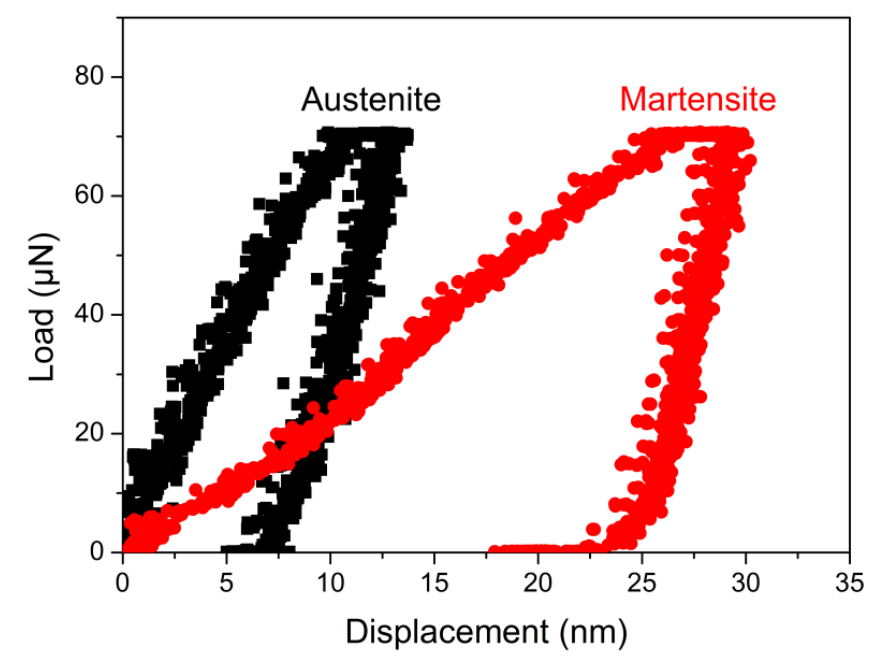

Figure 6-9 Load-displacement curves of martensite and austenite in the 12\% Mn alloy in the $500{ }^{\circ} \mathrm{C} / 10080 \mathrm{~min}$ state.

The representative hardness values of the $10 \% \mathrm{Mn}$ and $12 \% \mathrm{Mn}$ alloys in the $500{ }^{\circ} \mathrm{C}$ / 10080 min state are given in Figure 6-10. In the 10\% Mn alloy, the hardness of austenite and martensite are $8.5 \pm 1.0$ and $5.5 \pm 0.3 \mathrm{GPa}$, respectively; in the $12 \%$ Mn alloy, the hardness of austenite and martensite are 9.0 \pm 1.1 and $4.9 \pm 0.5 \mathrm{GPa}$, respectively. It should be noted that the variation of austenite hardness in both alloys is larger than that of martensite. 

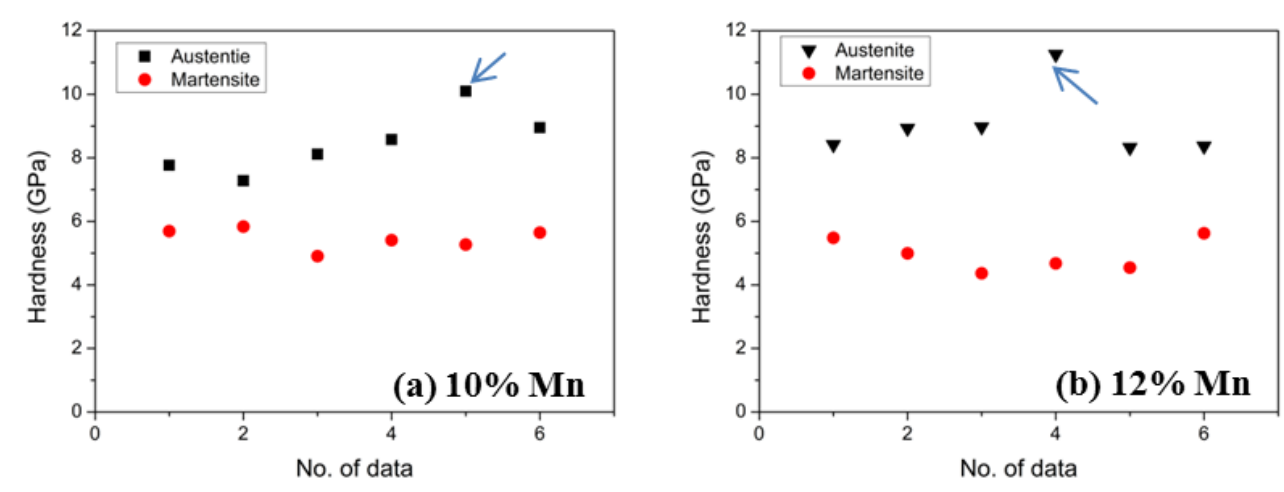

Figure 6-10 Hardness distribution of austenite and martensite phases in (a) 10\% Mn alloy, in $500{ }^{\circ} \mathrm{C} / 10080 \mathrm{~min}$ state and (b) $12 \% \mathrm{Mn}$ alloy, in $500{ }^{\circ} \mathrm{C} / 10080 \mathrm{~min}$ state.

The $P$ - $h$ curve of the indexed point in Figure 6-10(b) is representatively illustrated in Figure 6-11. A pop-in point near the top of the curve is visible. According to the study by $\mathrm{He}$ et al. [188], the pop-in point may correspond to a phase transformation. It is believed there is a critical load for the martensitic transformation $\left(P_{c}\right)$, which is regarded as an indication of the mechanical stability of single austenite grain [188]. As the load applied in this study was small, most indentations on austenite did not lead to the martensitic transformation. Even in the indentation displayed in Figure 6-11, the martensitic transformation occurred when just approaching the maximum load, so the deformation behaviour of transformed martensite was not revealed. Otherwise a martensitic transformation strengthening is expected after the pop-in point. Furnemont et al. [245] reported that the slope of $P$ - $h$ curve after the pop-in point should be higher than that before the point. The martensitic transformation strengthening can be interpreted by dispersion hardening mechanism [248]. Accompanying with the phase transformation, a geometrically necessary dislocation (GND) prismatic loops [249] generate within the plastic zone close to the transformed martensite, leading to the increase of dislocation density and thereby strengthening the austenite phase [250]. During the loading, the untransformed austenite can be further strengthened when the GND prismatic loops bypass martensite and leave dislocation debris around the martensite. In terms of the martensite, the transformed martensite may have subjected severe deformation during the indentation which also contributes to the increase of hardness as higher 
load is required to deform martensite. Another mechanism related to the pop-in point in $P$ - $h$ curves was proposed by Wo et al. [251]. According to these researchers, the high-angle grain boundaries of austenite phase can prevent dislocations from passing through grain boundaries and thus leads to the grain boundary strengthening. Therefore, when the indentation position is just located at high-angle grain boundaries, a higher hardness than that on lower-angle grain boundaries or within plastic zone is expected. Both these two mechanisms may explain the pop-in point shown in Figure 6-11.

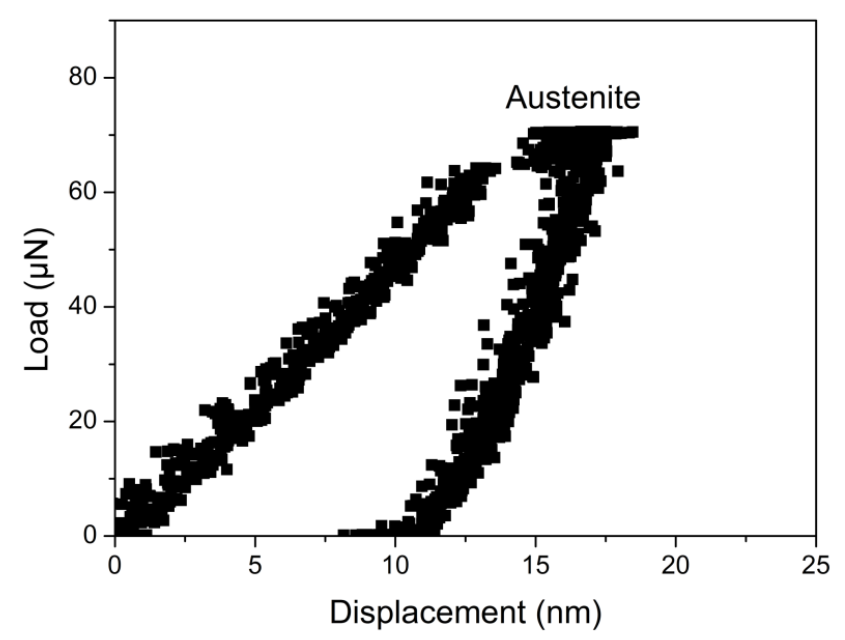

Figure 6-11 Load-displacement curve recording the martensitic transformation of an austenite grain.

\subsection{Discussion}

In the following sections the evolution of the grain size, chemical composition and volume fraction of reverted austenite will be discussed. Based on the discussion, the formation mechanism of reverted austenite is determined. Then the discussion will focus on the relationship between the mechanical stability of reverted austenite and its microstructure. 


\subsubsection{The size of reverted austenite grains}

The average sizes of reverted austenite formed under different aging conditions were measured based on the SEM observation. Although the austenite reversion occurred in the very beginning of aging process, the size evolution of reverted austenite nanolayers in the early aging stage was difficult to capture due to the resolution limits of the microscope, and therefore, the discussion inevitably focuses on the size of lath-like reverted austenite in the over-aged condition. On the other hand, as lathlike austenite has a wide range of length and the length of lath-like reverted austenite may vary with different orientations, it is difficult to measure the average length accurately. Therefore, only the width of reverted austenite is analysed here. In Figure 6-12 and Figure 6-13, the width of reverted austenite was averaged from at least 80 measurements.

As shown in Figure 6-12, at $500{ }^{\circ} \mathrm{C}$ there was a slow increase in the average width of austenite grains with aging time for both the $10 \% \mathrm{Mn}$ and $12 \% \mathrm{Mn}$ alloys from 2880 min to $10080 \mathrm{~min}$ (Figure 6-12). This limited increase in the width is attributed to two reasons: (i) the decelerating kinetics of austenite growth for the over-aged stage, as a result of the slow diffusion of Mn atoms (EDS analyses revealed that the austenite formed at this stage had a high Mn concentration ( 24 at.\%)) [27]; (ii) thermodynamic resistance to coalescence with adjacent reverted austenite grains as discussed in Section 2.4.2. In addition, the influence of the Mn content of alloy on the austenite width was not evident, and the widths of reverted austenite seemed to be irrelevant to the Mn content and are almost the same between the $10 \% \mathrm{Mn}$ and $12 \%$ Mn alloys (Figure 6-12). 


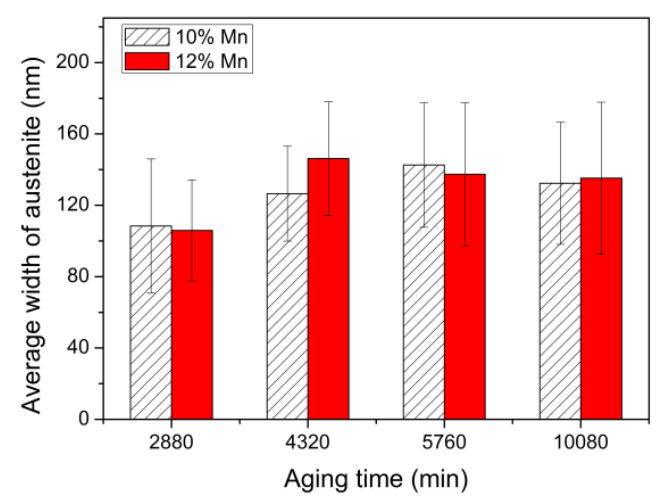

Figure 6-12 Average width of lath-like reverted austenite after aging at $500{ }^{\circ} \mathrm{C}$ for different times in the $10 \% \mathrm{Mn}$ and $12 \% \mathrm{Mn}$ alloys.

In contrast to the aging duration and alloying composition, the aging temperature had a more evident influence on the width of reverted austenite as presented in Figure 6-13, which showed an approximately linear increase with the aging temperature. It is not difficult to understand as the higher driving force for Mn diffusion at elevated temperature promoted the growth of reverted austenite.

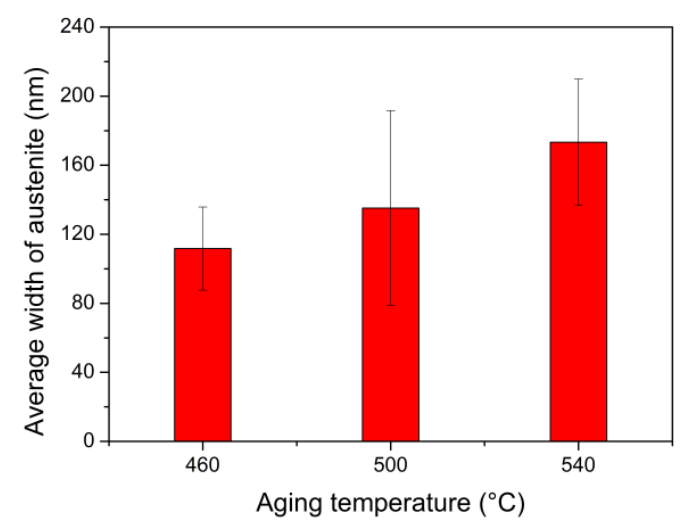

Figure 6-13 Average width of reverted austenite aged at different temperatures for 10080 $\min$ in the $12 \%$ Mn alloy.

\subsubsection{Chemical composition of reverted austenite}

In this study, Mo- and Ti-rich austenite was observed in some samples (see Table 61). This phenomenon is contradictory to the common knowledge that $\mathrm{Ti}$ is regarded 
as ferrite stabilizing elements. A similar finding on the M-A-M (M: martensite, A: austenite) region using APT was reported in a 9 wt.\% Mn maraging steel by Raabe et al. [50]. They summarized the experimental grain boundary segregation data of various elements [50] and reported that the grain boundary enrichment factor of $\mathrm{Mn}$ is $2.3, \mathrm{Ni}$ is $1.7, \mathrm{Mo}$ is 2.3 and $\mathrm{Ti}$ is 5.2 . Therefore, in the current study, it is assumed that the $\mathrm{Ti}$ and Mo-rich austenite nanolayers in the 7\% Mn and 10\% Mn alloys was due to the fact that most of the austenite phase was formed along the prior austenite grain boundaries and martensite packet/lath boundaries. These interfaces were also the preferential segregation sites for Mo and Ti. As Ti has a higher grain boundary enrichment factor and a faster diffusivity, the notably high Ti concentrations in the austenite cannot be avoided. Subsequently, continuous diffusion of Mn led to the newly-formed Mn-rich austenite on top of the preceding Ti, Mo-rich grain boundaries. On the other hand, in this type of austenite, there was no segregation of $\mathrm{Ti}$ and Mo, so these austenite grains were observed with higher Mn concentration. Another reason relating to the $\mathrm{Ti}$ and Mo enrichment in austenite was proposed by Schnitzer et al. who suggested that the high solubility of $\mathrm{C}$ in austenite drove the formation of Ti- and Mo-rich carbides within the austenite phase [147]. However in the present Mn-based maraging steels, as the nominal carbon content of the alloys was very low (0.02-0.03 wt.\%) and no carbide was observed, it is not likely that the limited carbon atoms within austenite could lead to such high concentrations of $\mathrm{Ti}$ and Mo in austenite.

The equilibrium binary Fe-Mn phase diagram is presented in Figure 6-14 to study the effects of temperature and nominal composition on the Mn concentration of austenite. According to the Fe-Mn phase diagram, the equilibrium compositions of $\mathrm{Fe}-10 \mathrm{Mn}$ alloy at $500{ }^{\circ} \mathrm{C}$ are ferrite with 3.7 at.\% $\mathrm{Mn}$ and austenite with 21.3 at.\% Mn. Increasing the Mn content of the alloy does not lead to the composition change in the ferrite or austenite, but different aging temperatures result in the variation of austenite composition. For example at $460{ }^{\circ} \mathrm{C}$, ferrite with 3.9 at.\% $\mathrm{Mn}$ and austenite with 25.0 at.\% Mn can be formed regardless of the nominal Mn content. 


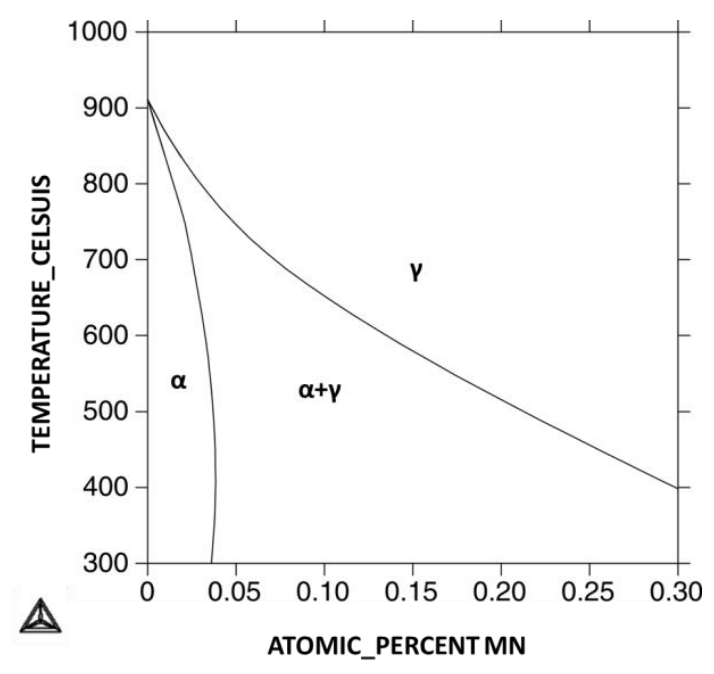

Figure 6-14 Equilibrium phase diagram of the Fe-Mn system.

The chemical composition profiles obtained by TEM-EDS are partially consistent with Fe-Mn phase diagram. The segregation of $\mathrm{Ti}$ and Mo on grain boundaries resulted in the Mn concentration of austenite nanolayers being far from that indicated by the equilibrium phase diagram. Although the phase diagram suggests that the $\mathrm{Mn}$ concentration of austenite is independent on the nominal Mn content of the alloy, experimental results reveal that higher Mn content contributed to a fast kinetics for the formation of reverted austenite and thus higher Mn concentration of reverted austenite for the same aging conditions. Otherwise, the chemical composition of ferrite and lath-like austenite exhibited good agreement with the phase diagram.

It is worth discussing the thermodynamic possibility of the austenite formation with relatively low Mn concentration along the grain boundaries in the initial stage of aging. Wilson and his colleagues [121][122] worked on the grain boundary segregation of $\mathrm{Mn}$ in a Fe-8Mn alloys at $450{ }^{\circ} \mathrm{C}$ and revealed by Auger spectroscopy that about 18 at.\% Mn enrichment at the grain boundaries upon aging at $450{ }^{\circ} \mathrm{C}$ for 10-12 min. They suggested the Mn segregation to prior austenite grain boundaries supported the initial formation of reverted austenite at such sites. In our study, the Mn concentration of austenite nanolayers formed in the very beginning of aging was even lower owing to the Ti and Mo segregation at the grain boundaries. Raabe et al. [50] calculated the chemical driving force for the local austenite formation at $450{ }^{\circ} \mathrm{C}$ in different conditions. In the binary Fe-Mn system, a minimum 13.3 at.\% $\mathrm{Mn}$ is required to approach the critical chemical driving force for austenite reversion. If 
other alloying elements (Mo, Ti and $\mathrm{Si}$ ) are included, the Mn concentration above 12.4 at.\% Mn has the potential to form austenite. In the case with the local segregation of ferrite stabilizing elements, higher Mn concentration (above 15.3 at.\%) is needed for the phase transformation to austenite. However, structural defects such as interface disorder, which is a significant factor, are not taken into consideration in the above calculations. Therefore, Raabe et al. [50] used a phase field model to model both the segregation and phase transformation, which revealed a high driving force for the formation of austenite at grain boundaries. The low nucleation energy at interface $\left(0.5-1 \mathrm{eV}\right.$ based on 24 at.\% Mn concentration at $\left.450{ }^{\circ} \mathrm{C}\right)$ is attributed to not only the enrichment of Mn at grain boundaries, but also the low energy of K-S austenite/martensite interface and specifically, the high elastic relaxation energy of martensite induced by phase transformation.

In the present study, as the austenite reversion at the interface would be suppressed by the segregation of ferrite stabilizing elements ( $\mathrm{Ti}$ and $\mathrm{Mo}$ ), the critical $\mathrm{Mn}$ concentration for austenite reversion at the interface should be higher than that calculated on the basis of the nominal chemical composition. However, in view of the low nucleation energy at the interface due to structural defects, austenite reversion can still be realized with relatively low $\mathrm{Mn}$ concentrations at the grain boundaries. A detailed discussion on the formation of reverted austenite will be given in Section 6.6.4.

In addition, Raabe et al. performed an APT analysis on the M-A-M interface where retained austenite served as a template for further austenite reversion to study the difference between retained austenite and reverted austenite in maraging TRIP steels [50]. They reported that this special M-A-M interface presented a different compositional profile from the self-nucleated reverted austenite. Retained austenite in the centre region of $\mathrm{Mn}$ profile revealed a Mn concentration of 12 at.\%, while the reverted austenite formed on top of it had a Mn concentration of $\sim 25$ at.\%. This indicates that the Mn concentration of reverted austenite was much higher than that of retained austenite. 


\subsubsection{The volume fraction of reverted austenite}

According to the Fe-Mn phase diagram, a sufficient long-time aging at $500{ }^{\circ} \mathrm{C}$ allows the formation of up to $36 \mathrm{vol} . \%$ austenite for a $10 \% \mathrm{Mn}$ alloy. Increasing the Mn content of the alloy leads to an increase of the austenite fraction, so that at equilibrium, the volume fraction of austenite is 47.5 vol.\% for the $12 \% \mathrm{Mn}$ alloy. Different aging temperatures also result in the variation in composition and volume fraction of austenite. A summary of the equilibrium composition and volume fractions of phase as a function of aging temperature is presented in Table 6-4.

Table 6-4 A summary of the equilibrium Mn concentration (at.\%) and volume fraction (vol.\%) of austenite and ferrite phases at different aging temperatures based on the Fe-Mn phase diagram in Figure 6-14.

\begin{tabular}{rccccccc}
\hline & & \multicolumn{2}{c}{$7 \% \mathrm{Mn}$} & \multicolumn{2}{c}{$10 \% \mathrm{Mn}$} & \multicolumn{2}{c}{$12 \% \mathrm{Mn}$} \\
\cline { 3 - 8 } & & at.\% & vol.\% & at.\% & vol.\% & at.\% & vol.\% \\
\hline \multirow{2}{*}{$420{ }^{\circ} \mathrm{C}$} & Austenite & 28.4 & 13.1 & 28.4 & 25.0 & 28.4 & 33.3 \\
& Ferrite & 4.0 & 86.9 & 4.0 & 75.0 & 4.0 & 66.7 \\
& Austenite & 25.0 & 15.4 & 25.0 & 29.3 & 25.0 & 39.0 \\
$460{ }^{\circ} \mathrm{C}$ & Ferrite & 3.9 & 84.6 & 3.9 & 70.7 & 3.9 & 61.0 \\
& Austenite & 21.3 & 18.7 & 21.3 & 36.0 & 21.3 & 47.5 \\
\hline \multirow{2}{*}{$500{ }^{\circ} \mathrm{C}$} & Ferrite & 3.7 & 81.3 & 3.7 & 64.0 & 3.7 & 52.5 \\
& & & & & & & \\
\hline
\end{tabular}

The experimental results of the volume fraction of reverted austenite (Table 6-3) are compared to the data from the equilibrium Fe-Mn phase diagram (Table 6-4). It is obvious that the maximum austenite phase fractions (up to $\sim 10$ vol. $\%$ for $10 \% \mathrm{Mn}$ alloy aged at $500{ }^{\circ} \mathrm{C}$; up to $\sim 24$ vol. $\%$ for $12 \% \mathrm{Mn}$ alloy aged at $500{ }^{\circ} \mathrm{C}$ ) are significantly lower than the values predicted by the Fe-Mn phase diagram ( 36.0 vol.\% for $10 \% \mathrm{Mn}$ alloy at $500{ }^{\circ} \mathrm{C}$; $\sim 4.5 \mathrm{vol} . \%$ for $12 \% \mathrm{Mn}$ alloy at $500{ }^{\circ} \mathrm{C}$ ). This may be attributed to the fact that a small amount of reverted austenite transformed to $\varepsilon$ martensite during cooling. In addition, the addition of $\mathrm{Ti}$ and $\mathrm{Al}$ could raise the $A_{e}^{3}$ 
and reduced the maximum volume fraction of austenite. The segregation of $\mathrm{Ti}$ and Mo at grain boundaries is considered to suppress the formation of reverted austenite as well.

\subsubsection{The formation mechanism of reverted austenite}

As revealed in Section 6.2, in the initial aging stage, austenite nanolayers were preferentially formed along prior austenite grain boundaries and martensite packet boundaries due to low nucleation energy at interface. Further aging led to those austenite nanolayers gradually growing to film-like and then lath-like austenite. In some cases (e.g. 12\% Mn alloy after prolonged/high-temperature aging), lath-like reverted austenite was also found within martensite laths. In addition, as both the prior austenite grains and martensite packets were largely refined due to higher $\mathrm{Mn}$ content (see Figure 4-2), the 12\% Mn alloy provided more nucleation sites for austenite formation and this partially explains why the austenite reversion of $12 \%$ Mn alloy kinetically exceed that of the other two alloys.

As discussed in Section 2.4.2, the formation of reverted austenite is generally considered to be dominated by a diffusion process [27][193][194]. but some researchers proposed that austenite reversion is shear-dominated and assisted by a prior or simultaneous diffusion mechanism [52][146]. In order to study the formation mechanism of reverted austenite in Mn-based maraging steels, the results of reverted austenite fraction were analysed. As the experimental study has confirmed that there was no retained austenite formed by quenching from solution heat treatment, the entire austenite detected in the aged samples is regarded as reverted austenite.

It is worth noting that the complexity of austenite reversion process in maraging steels, such as the competition with precipitation and the presence of coherency strains, may have led to the experimental results that are contradictory to the common knowledge [54]. On the other hand, previous microstructural observation has demonstrated that the formation of precipitates and reverted austenite started from the very beginning of aging simultaneously, and the computer simulation also indicates that the dissolution of precipitates is not necessary for the initial driving force for austenite reversion in maraging steels [147]. Therefore, it is assumed that 
the formation of precipitates and reverted austenite are independent processes in this study. It should be highlighted that this analysis of the kinetics is only regarded as a semi-quantitative description of austenite reversion.

When the austenite reversion process is diffusion-controlled, its activation energy $Q$, can be calculated by the following equation [147]:

$$
\ln (t)=\frac{Q}{R T}+c
$$

where $t$ is the time to form certain volume fraction of reverted austenite, $T$ is the specific temperature at which austenite reversion occurs, and $c$ is the constant. In the current study, the analyses of austenite formation were performed on the $10 \%$ and $12 \%$ Mn alloys by measuring the time required to form the certain amount of reverted austenite at $460{ }^{\circ} \mathrm{C}, 500{ }^{\circ} \mathrm{C}$ and $540{ }^{\circ} \mathrm{C}$, respectively. The volume fraction for Equation (6.1) was set as 4 vol.\% for the $10 \% \mathrm{Mn}$ alloy and 9 vol.\% for the $12 \% \mathrm{Mn}$ alloy. These figures were chosen as the point before which $\varepsilon$-martensite was formed. Thus, the time to attain the certain amounts of reverted austenite as a function of aging temperature could be determined for both alloys. The results are presented in Figure 6-15 and a linear correlation is found between $1 / T^{*} 1000$ and $\ln (t)$. Based on the slope of fitted line, the activation energy for austenite reversion was estimated as $63.5 \mathrm{~kJ} / \mathrm{mol}$ for the $10 \% \mathrm{Mn}$ alloy and $85.7 \mathrm{~kJ} / \mathrm{mol}$ for the $12 \% \mathrm{Mn}$ alloy. These values are significantly lower than those reported in conventional maraging steels and the activation energy for $\mathrm{Mn}$ diffusion in pure iron as well. The activation energy for Mn diffusion in Fe is reported to be 219.8-261.7 kJ/mol [252]. Nakagawa et al. [20] reported the activation energy for austenite reversion was about 240 $\mathrm{kJ} / \mathrm{mol}$ in a $1.8 \mathrm{Cu}-7.3 \mathrm{Ni}-15.9 \mathrm{Cr}-1.2 \mathrm{Mo}$ steel. Schntizer et al. [147] reported the activation energy was $234 \pm 20 \mathrm{~kJ} / \mathrm{mol}$ in PH 13-8 Mo maraging steels. However in this study, the volume fraction of austenite chosen for the calculation is very low, which means that the calculation is mainly based on the early stage of austenite reversion. Therefore, it is considered that the austenite reversion in the early aging stage was not controlled by Mn diffusion. On the other hand, as the austenite reversion occurred at the onset of aging and the compositional change was small, the formation of reverted austenite is proposed to be a shear mechanism accompanied by a small amount of Mn diffusion. 


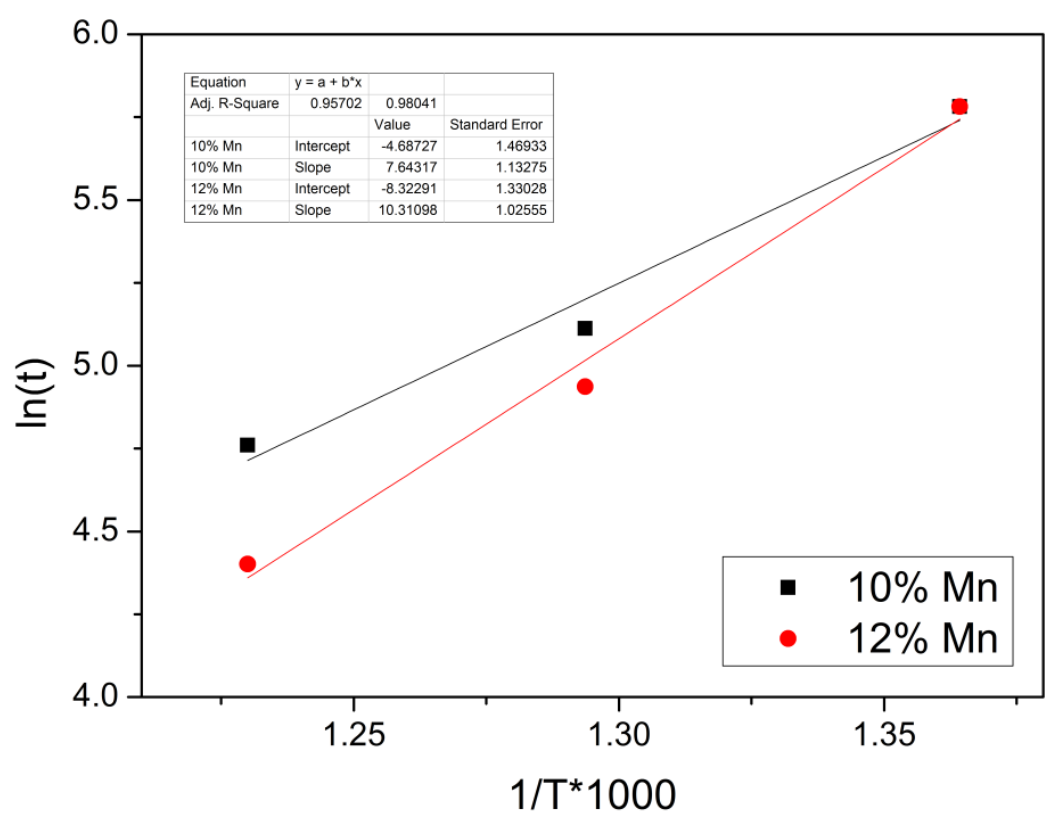

Figure 6-15 Arrhenius plot for the determination of the activation energy of austenite formation in the $10 \% \mathrm{Mn}$ and $12 \% \mathrm{Mn}$ alloy.

On the other hand, our TEM-EDS investigation on austenite reversion reveals that the $\mathrm{Mn}$ concentration of lath-like reverted austenite in the later aging stage ( 24 at.\%) was much higher than that of austenite nanolayers formed in the early aging stage ( 12 at.\%). The high concentration of Mn indicates that Mn was indeed involved in the formation of reverted austenite. Therefore, the variation of Mn concentration is considered as the result of the transition of austenite formation mechanism from diffusionless-dominant mechanism to diffusion-dominant mechanism.

Based on the experimental results and discussion presented above, the sequence for reverted austenite formation in these Mn-based maraging steels is proposed as following:

1) The nucleation of reverted austenite at prior austenite grain boundaries and martensite packet boundaries occurred at the onset of aging followed by the nucleation of austenite within martensite laths. The nucleation process was governed by a shear mechanism assisted by a prior or simultaneous diffusion of a small amount of Mn. Due to the structural disorder at interface, there is a higher 
driving force for the segregation of $\mathrm{Mn}$ and phase transformation, which made the rapid nucleation of reverted austenite at grain boundaries easier. While the nucleation of reverted austenite within martensite laths needed higher Mn segregation and hence was kinetically slower.

2) During the further aging process, the accumulated $\mathrm{Mn}$ segregation to grain boundaries led to the austenite nanolayers growing to lath-like reverted austenite, which means the lateral growth of austenite was mainly supported by the diffusion of $\mathrm{Mn}$. As the diffusion rate of $\mathrm{Mn}$ in the martensite matrix was relatively low, the diffusion-controlled austenite growth was fairly slow. In addition, the thermodynamic resistance to coalescence between adjacent reverted austenite is another reason why the reverted austenite could maintain nano-scale dimensions for a long time.

\subsubsection{The stability of reverted austenite}

As discussed in Section 2.4.1, studies on reverted austenite suggest that the size effect and enriched Mn concentration of austenite grains are the two main intrinsic factors that affect the stability of reverted austenite.

\section{- Chemical composition}

Yen et al. [26] stated that the essential concept of austenite engineering in Mn-based TRIP steels is to tailor the Mn concentration in austenite in order to attain metastable austenite after heat treatment. This metastable austenite can therefore lead to the TRIP effect which enables strain hardening.

The martensite transformation start temperature $\left(M_{S}\right)$ is regarded as a parameter that directly controls the stability of the austenite. The $M_{S}$ temperature based purely on

the chemical compositions is designated $M_{S}^{0}$. Therefore, the $M_{S}^{0}$ of the $\alpha^{\prime}$-martensite can be calculated by the following equation [253]:

$$
M_{s}^{0}\left({ }^{\circ} \mathrm{C}\right)=545-330 \mathrm{C}+2 \mathrm{Al}-23 \mathrm{Mn}-5 \mathrm{Mo}-13 \mathrm{Ni}+3 \mathrm{Ti}
$$

where the elements stand for their concentrations in wt.\%. The equation is simplified to only present the primary elements involved in this study. 
According to Equation (6.2), the relationship between the austenite stability and chemical composition is quite straightforward. C and Mn significantly contribute to the stability of austenite while the $\mathrm{Al}$ and $\mathrm{Ti}$ additions lower the austenite stability. The influence of Ti and Mo enrichment on the initial formation of reverted austenite along the grain boundaries was discussed in Section 6.6.2. Here we mainly focus on the effects of Mn concentration on the austenite stability.

Figure 6-16 presents the average hardness values of austenite and martensite in various aged samples. As displayed in Figure 6-16(a), the austenite hardness of the $12 \% \mathrm{Mn}$ alloy aged at $500{ }^{\circ} \mathrm{C}$ for $10080 \mathrm{~min}$ was higher than that of the $10 \% \mathrm{Mn}$ alloy for the same aging condition, which is believed to be mainly attributed to the higher $\mathrm{Mn}$ concentration of austenite in the $12 \% \mathrm{Mn}$ alloy (revealed by previous TEM-EDS analyses). On the other hand, the martensite hardness of the $12 \% \mathrm{Mn}$ alloy was slightly lower than that of the $10 \%$ Mn alloy. This might be a result of the faster kinetics of the coarsening of precipitates and softening of martensite matrix in the $12 \% \mathrm{Mn}$ alloy. In terms of the effect of aging temperature, it is clearly shown in Figure 6-16(b) that the hardness of both the austenite and martensite decreased as the aging temperature increased. The decrease of martensite hardness mainly resulted from the coarsening of precipitates and softening of martensite matrix at higher temperature. While the reverse relationship between the austenite hardness and aging temperature is considered to be related to the decreased Mn concentration of reverted austenite with the increase of aging temperature.

It should be noted that the above discussion is based on the average statistics. Due to the relatively low diffusion rate of $\mathrm{Mn}$, the Mn concentration of each single austenite grain may vary from grain to grain, leading to the variation of austenite hardness which manifests the large scatter in Figure 6-10. Therefore, a detailed analysis on the hardness and $\mathrm{Mn}$ concentration of each single austenite grain may be a more direct method to explore the relationship between Mn concentration and the mechanical stability of austenite phase. 

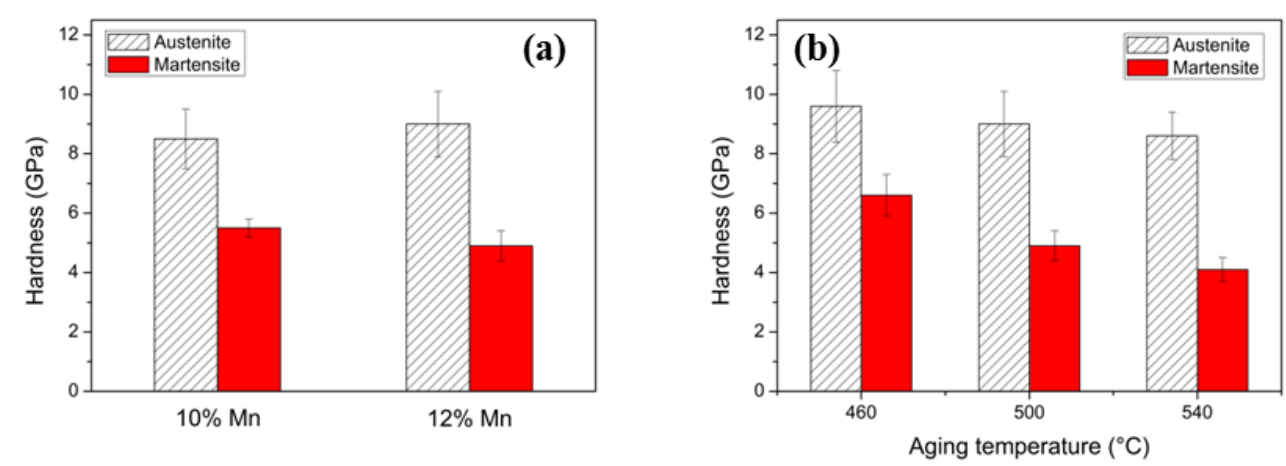

Figure 6-16 (a) The average hardness of austenite and martensite in the 10\% Mn and 12\% Mn alloys aged at $500{ }^{\circ} \mathrm{C}$ for $10080 \mathrm{~min}$; (b) the hardness of austenite and martensite in the $12 \% \mathrm{Mn}$ alloy as a function of aging temperature.

\section{- Austenite grain size}

It is generally accepted that the austenite with a small grain size has a high stability [154][34][158][159][162][163]. Although the interface is always a good site for heterogeneous nucleation, phase transformation is suppressed in materials with small grain sized even when there is a strong thermodynamic driving force. This has been shown to be true for both metals and ceramics.

In contrast, Wang et al. [171] reported a 'smaller is less stable' effect of austenite in a 9Mn maraging steel. They stated that the mechanical twinning was activated in larger austenite grains within which the deformation substructures stabilized grains. While for smaller austenite grains, mechanical twinning was less likely to occur. Another study by Wang et al. [166] proposed it is easier for the outer layer of reverted austenite to transforms into martensite during deformation owing to the higher stress and lower concentration of austenite stabilizing elements. As the fraction of outer layer region is smaller the austenite grain with a larger dimension, the larger reverted austenite grains are more stable than the smaller ones. However, our study on Mn-based maraging steels did not find any evidence of mechanical twinning in the reverted austenite. Besides, the nanoindentation analysis confirmed that in the over-aged state when reverted austenite could be detected by AFM, reverted austenite was harder than the adjacent martensite matrix. On the other hand, the concentration variation between the outer layer and centre can be negligible 
when the grain size of reverted austenite was so fine (e.g. < $200 \mathrm{~nm}$ in this study). Therefore, the above two 'smaller is less stable' effects apparently cannot be applied in the present work.

In this study, since the reverted austenite exhibited an elongated shape with a wide range of lengths, it was not possible to obtain an accurate equivalent size of reverted austenite grains. On the other hand, lath-like reverted austenite exhibited a strong resistance to coarsening in the lateral direction, maintaining a size in the range 70$200 \mathrm{~nm}$ under various aging conditions. Due to the resolution limit, nanoindentation study did not reveal a direct relationship between the stability and austenite size in this study. Although higher austenite hardness was found at lower aging temperature (Figure 6-12 (b)) where the average austenite size was smaller (Figure 6-13), it cannot be concluded that the smaller size of reverted austenite was the reason for the higher mechanical stability. Other factors, such as the higher Mn concentration at lower aging temperature (Table 6-4), have been demonstrated to have a more evident effect on the mechanical stability. Therefore, detailed investigation by performing nanoindentation on individual single austenite grain with measurable sizes and known Mn concentration is needed to understand the relationship between the size and the stability of austenite.

\subsection{Summary}

1. Owing to the high grain boundary enrichment factor of Ti and Mo, Ti and Morich austenite along the grain boundaries was observed in the initial aging stage. The high concentrations of Ti and Mo gradually vanished with the enrichment of Mn during the succeeding aging process.

2. The experimental chemical composition profile of reverted austenite was partially consistent with that given by the Fe-Mn phase diagram. The segregation of Ti and Mo on grain boundaries resulted in the Mn concentration of austenite nanolayers in the initial aging stage being far from the value indicated by the equilibrium phase diagram. Otherwise, the chemical composition of ferrite and lath-like austenite exhibited good agreement with the phase diagram. The TEM-EDS analyses revealed the Mn concentration of 
lath-like austenite was at the level of $\sim 24$ at.\% which is much higher than that of retained austenite (8-12 at.\%) reported in conventional Mn-based TRIP or Q\&P steels [27][154][34][50]. The volume fraction of reverted austenite increased almost linearly with the logarithm of aging time until it was saturated. The experimental results of the equilibrium austenite fractions (e.g. $\sim 10$ vol.\% for $10 \% \mathrm{Mn}$ alloy aged at $500{ }^{\circ} \mathrm{C}$ and $\sim 24$ vol. $\%$ for $12 \% \mathrm{Mn}$ alloy aged at $500{ }^{\circ} \mathrm{C}$ ) were significantly lower than the values predicted by the Fe-Mn phase diagram (36.0 vol.\% for Fe-10Mn at $500{ }^{\circ} \mathrm{C}$ and $\sim 47.5$ vol.\% for Fe-12Mn at $500{ }^{\circ} \mathrm{C}$ ). This is mainly attributed to the fact that an amount of reverted austenite transformed to $\varepsilon$-martensite during cooling. The addition of $\mathrm{Ti}$ and $\mathrm{Al}$ may be another reason for the discrepancy.

3. The formation of reverted austenite nanolayers initiated at the onset of aging by a diffusionless shear mechanism accompanied by a small amount of $\mathrm{Mn}$ diffusion like bainite. The nucleation of reverted austenite within martensite laths was kinetically slower due to a lower driving force for phase transformation. In the following aging process, the accumulated $\mathrm{Mn}$ segregation to the newly-formed austenite/martensite grain boundaries led to the austenite nanolayers growing to lath-like reverted austenite, which means the lateral growth of austenite in the later aging stage was supported by the diffusion of Mn.

4. As the diffusion rate of $\mathrm{Mn}$ in the martensite matrix was relatively low, the diffusion-controlled austenite growth was fairly slow. In addition, the thermodynamic resistance to coalescence between adjacent reverted austenite grains is another reason why the width of reverted austenite could maintain in the range 70-200 $\mathrm{nm}$ for a long time. The width of reverted austenite was not closely associated with the nominal Mn content of the alloy, but it is found that the width increased with the increase of aging temperature owing to the higher driving force for Mn diffusion at higher temperature.

5. Most of the reverted austenite in Mn-based maraging steels exhibited a K-S or $\mathrm{N}$ orientation relationship with the residual martensite. It is believed that both the K-S and $\mathrm{N}$ orientation relationships contributed to the mechanical stability of reverted austenite. 
6. Nanoindentation testing revealed the high stability of reverted austenite in Mnbased maraging steels was mainly attributed to the high Mn concentration of austenite. The nano-size of reverted austenite was also considered to be responsible for the high stability, but more evidence is needed to support this viewpoint. 


\section{Chapter 7 Strengthening mechanisms of $\mathrm{Mn}$ - based maraging steels}

Table 7-1 summarizes the tensile properties of Mn-based maraging steels in this study together with other research results on medium Mn steels (mainly with 5-7 wt.\% Mn). As shown in Table 7-1, impressive tensile properties have been reported in other Mn-based steels subjected to various processing paths. Nevertheless, in view of the low carbon content $(<0.03 \mathrm{wt} . \%)$ of the steels in this work, the tensile strength of 9-12\% Mn steels are still remarkable compared to steels with the same level of carbon content in Table 7-1. It is proposed that the high UTS is attributed to the joint effect of the precipitation strengthening of $\mathrm{Ni}_{2} \mathrm{TiAl}$ intermetallic phase and the work hardening of reverted austenite. On the other hand, the ductility of the $10 \%$ and $12 \%$ Mn alloys in this study is relatively mediocre. One possible reason is the relatively lower austenite fraction than other alloys. Another reason we speculated is associated with the high mechanical stability of reverted austenite. In this chapter, the strengthening mechanisms of Mn-based maraging steels will be investigated and based on the discussion, the optimal microstructure and relevant processing paths to obtain the microstructure will be determined with the aim to achieve better mechanical properties.

The strengthening mechanism for steels is complex and generally it can be expressed as:

$$
\sigma_{y}=\sigma_{B}+\sigma_{S S}+\sigma_{G R}+\sigma_{P H}+\sigma_{\text {dislocation }}
$$

where $\sigma_{y}$ is the total yield strength, $\sigma_{B}$ is the yield strength of base steel, $\sigma_{S S}$ is the solid-solution strengthening, $\sigma_{G R}$ is the grain refinement strengthening, $\sigma_{P H}$ is the precipitation strengthening and $\sigma_{\text {dislocation }}$ is the dislocation strengthening. In the following sections, the dominant strengthening mechanism in different heat treatment conditions will be discussed. 
Table 7-1 Summary of the tensile properties of Mn-based steels.

\begin{tabular}{|c|c|c|c|c|c|c|}
\hline Materials (wt.\%) & Heat treatment & $V_{\gamma}(\operatorname{vol} . \%)$ & YS (MPa) & UTS (MPa) & TE (\%) & Ref. \\
\hline Fe-0.015C-10Mn-2Ni-1.2Mo-1Al-0.8Ti & $500^{\circ} \mathrm{C} / 5760 \mathrm{~min}, \mathrm{WQ}$ & 10.4 & 860 & 1062 & 17 & $*$ \\
\hline $\mathrm{Fe}-0.022 \mathrm{C}-11.8 \mathrm{Mn}-2 \mathrm{Ni}-1.2 \mathrm{Mo}-1 \mathrm{Al}-0.8 \mathrm{Ti}$ & $500{ }^{\circ} \mathrm{C} / 5760 \mathrm{~min}, \mathrm{WQ}$ & 24.6 & 870 & 1120 & 18.4 & $*$ \\
\hline $\mathrm{Fe}-0.007 \mathrm{C}-8.86 \mathrm{Mn}-2.0 \mathrm{Ni}-1.07 \mathrm{Mo}-1.04 \mathrm{Ti}$ & $450{ }^{\circ} \mathrm{C} / 48 \mathrm{~h}$ & - & 984 & $\sim 1000$ & $\sim 15$ & {$[18][26]$} \\
\hline $\mathrm{Fe}-0.007 \mathrm{C}-8.86 \mathrm{Mn}-2.0 \mathrm{Ni}-1.07 \mathrm{Mo}-1.04 \mathrm{Ti}$ & $450{ }^{\circ} \mathrm{C} / 48 \mathrm{~h}^{[\mathrm{a}]}$ & - & 978 & 1011 & 12.7 & {$[18][26]$} \\
\hline $\mathrm{Fe}-0.01 \mathrm{C}-11.9 \mathrm{Mn}-2.06 \mathrm{Ni}-1.12 \mathrm{Mo}-1.09 \mathrm{Ti}$ & $450{ }^{\circ} \mathrm{C} / 48 \mathrm{~h}$ & - & 1142 & $\sim 1300$ & 21 & {$[18][26]$} \\
\hline $\mathrm{Fe}-0.01 \mathrm{C}-11.9 \mathrm{Mn}-2.06 \mathrm{Ni}-1.12 \mathrm{Mo}-1.09 \mathrm{Ti}$ & $450{ }^{\circ} \mathrm{C} / 48 \mathrm{~h}^{\text {[a] }}$ & - & 1394 & $\sim 1500$ & $\sim 10$ & {$[18][26]$} \\
\hline $\mathrm{Fe}-0.01 \mathrm{C}-11.9 \mathrm{Mn}-2.06 \mathrm{Ni}-1.12 \mathrm{Mo}-1.09 \mathrm{Ti}$ & $650{ }^{\circ} \mathrm{C} / 144 \mathrm{~h}, \mathrm{AC}$ & 32.7 & $\sim 500$ & $\sim 980$ & $\sim 45$ & [27] \\
\hline $\mathrm{Fe}-0.11 \mathrm{C}-5.7 \mathrm{Mn}$ & $560^{\circ} \mathrm{C} / 1 \mathrm{~h}, \mathrm{AC}$ & 11 & 955 & 981 & 12 & [24] \\
\hline $\mathrm{Fe}-0.11 \mathrm{C}-5.7 \mathrm{Mn}$ & $520^{\circ} \mathrm{C} / 4 \mathrm{~h}, \mathrm{AC}$ & 10 & 934 & 956 & 19.5 & [24] \\
\hline $\mathrm{Fe}-0.11 \mathrm{C}-5.7 \mathrm{Mn}$ & $600^{\circ} \mathrm{C} / 1 \mathrm{~h}, \mathrm{AC}$ & 23 & 874 & 914 & 18.5 & [24] \\
\hline $\mathrm{Fe}-0.11 \mathrm{C}-5.7 \mathrm{Mn}$ & $640^{\circ} \mathrm{C} / 1 \mathrm{~h}, \mathrm{AC}$ & 30 & 798 & 1145 & 30.5 & {$[24]$} \\
\hline $\mathrm{Fe}-0.11 \mathrm{C}-5.7 \mathrm{Mn}$ & $600{ }^{\circ} \mathrm{C} / 16 \mathrm{~h}, \mathrm{AC}$ & 29 & 727 & 879 & 34 & {$[24]$} \\
\hline $\mathrm{Fe}-0.11 \mathrm{C}-5.7 \mathrm{Mn}$ & $640^{\circ} \mathrm{C} / 16 \mathrm{~h}, \mathrm{AC}$ & 30 & 87 & 174 & 18.5 & [24] \\
\hline
\end{tabular}




\begin{tabular}{|c|c|c|c|c|c|c|}
\hline $\mathrm{Fe}-0.04 \mathrm{C}-5 \mathrm{Mn}$ & $590{ }^{\circ} \mathrm{C} / 16 \mathrm{~h}^{[\mathrm{b}]}$ & 75.5 & 952 & 1049 & 39.5 & [13] \\
\hline $\mathrm{Fe}-0.045 \mathrm{C}-5 \mathrm{Mn}-3 \mathrm{Ni}$ & $590^{\circ} \mathrm{C} / 4 \mathrm{~h}^{[\mathrm{b}]}$ & 76.4 & 1104 & 1249 & 39.9 & {$[13]$} \\
\hline Fe-0.2C-4.72Mn & $650^{\circ} \mathrm{C} / 1 \mathrm{~min}, \mathrm{AC}$ & 4.54 & $\sim 830$ & $\sim 970$ & $\sim 20$ & [27] \\
\hline $\mathrm{Fe}-0.01 \mathrm{C}-7.09 \mathrm{Mn}$ & $575^{\circ} \mathrm{C} / 1$ week, WQ & 26 & 766 & 800 & 33 & {$[23]$} \\
\hline Fe-0.01C-7.09Mn & $625^{\circ} \mathrm{C} / 1$ week, WQ & 40 & 954 & 503 & 22 & {$[23]$} \\
\hline $\mathrm{Fe}-0.01 \mathrm{C}-7.09 \mathrm{Mn}$ & $675^{\circ} \mathrm{C} / 1$ week, WQ & 1.4 & 775 & 1368 & 7 & {$[23]$} \\
\hline Fe-0.08C-10.6Mn-0.36Si & $550{ }^{\circ} \mathrm{C} / 2 \mathrm{~h}, \mathrm{WQ}$ & 47 & 1240 & 1360 & 8.5 & [26] \\
\hline Fe-0.08C-10.6Mn-0.36Si & $610^{\circ} \mathrm{C} / 8 \mathrm{~min}, \mathrm{WQ}$ & 54 & 1080 & 1390 & 26.7 & [26] \\
\hline $\mathrm{Fe}-0.12 \mathrm{C}-4.6 \mathrm{Mn}-1.1 \mathrm{Al}-0.55 \mathrm{Si}$ & $720^{\circ} \mathrm{C} / 2 \mathrm{~min}, 10^{\circ} \mathrm{C} / \mathrm{s}^{[\mathrm{c}]}$ & 28 & 766 & 1204 & 12.9 & [16] \\
\hline $\mathrm{Fe}-0.12 \mathrm{C}-4.6 \mathrm{Mn}-1.1 \mathrm{Al}-0.55 \mathrm{Si}$ & $780{ }^{\circ} \mathrm{C} / 2 \mathrm{~min}, 10^{\circ} \mathrm{C} / \mathrm{s}^{[\mathrm{c}]}$ & 6 & 940 & 1461 & 6.2 & [16] \\
\hline $\mathrm{Fe}-0.12 \mathrm{C}-5.8 \mathrm{Mn}-3.1 \mathrm{Al}-0.47 \mathrm{Si}$ & $720^{\circ} \mathrm{C} / 2 \min ^{[\mathrm{c}]}$ & 26 & 814 & 854 & 14.7 & [16] \\
\hline $\mathrm{Fe}-0.12 \mathrm{C}-5.8 \mathrm{Mn}-3.1 \mathrm{Al}-0.47 \mathrm{Si}$ & $780{ }^{\circ} \mathrm{C} / 2 \mathrm{mi}^{[\mathrm{c}]}$ & 31 & 714 & 994 & 23.8 & [16] \\
\hline $\mathrm{Fe}-0.12 \mathrm{C}-5.8 \mathrm{Mn}-3.1 \mathrm{Al}-0.47 \mathrm{Si}$ & $840{ }^{\circ} \mathrm{C} / 2 \min ^{[c]}$ & 18 & 444 & 1161 & 11.2 & [16] \\
\hline Fe-0.05C-9Mn & $620^{\circ} \mathrm{C} / 600 \mathrm{~s}, \mathrm{FC}$ & 30 & 830 & 1034 & 27 & {$[254]$} \\
\hline $\mathrm{Fe}-0.05 \mathrm{C}-9 \mathrm{Mn}$ & $620^{\circ} \mathrm{C} / 600 \mathrm{~s}, \mathrm{FC}^{[\mathrm{d}]}$ & 37 & 1110 & 1193 & 25 & {$[254]$} \\
\hline
\end{tabular}




\begin{tabular}{ccccccc}
\hline Fe-0.1C-5.22Mn & $650{ }^{\circ} \mathrm{C} / 1 \mathrm{~h}, \mathrm{WQ}$ & - & - & 794 & 29 & {$[14]$} \\
Fe-0.1C-5.25Mn-2.09Si & $675{ }^{\circ} \mathrm{C} / 1 \mathrm{~h}, \mathrm{FC}$ & - & - & 1029 & 27 & {$[14]$} \\
Fe-0.01C-5Mn & $625^{\circ} \mathrm{C} / 3 \mathrm{~h}, \mathrm{WQ}$ & $\sim 4$ & $\sim 500$ & $\sim 800$ & $\sim 10$ & {$[255]$} \\
Fe-0.1C-5Mn & $650{ }^{\circ} \mathrm{C} / 3 \mathrm{~h}, \mathrm{WQ}$ & $\sim 30$ & $\sim 450$ & $\sim 1050$ & $\sim 24$ & {$[255]$} \\
$\mathrm{Fe}-0.4 \mathrm{C}-5 \mathrm{Mn}$ & $675{ }^{\circ} \mathrm{C} / 3 \mathrm{~h}, \mathrm{WQ}$ & $\sim 56$ & $\sim 550$ & $\sim 1700$ & $\sim 20$ & {$[255]$} \\
$\mathrm{Fe}-0.4 \mathrm{C}-5 \mathrm{Mn}$ & $675^{\circ} \mathrm{C} / 3 \mathrm{~h}, \mathrm{FC}$ & $\sim 15$ & $\sim 350$ & $\sim 1550$ & $\sim 10$ & {$[255]$}
\end{tabular}

AC: air cooling; WQ: water quenching; FC: furnace cooling.

* Tensile results of this work;

[a] Cold rolled before annealing

[b] four-step thermal cycling 2BT treatment, detail see [13]

[c] single-step heat treatment of intercritical annealing

[d] Cold rolled after hot rolling 


\subsection{The solid-solution and grain refinement strengthening}

As a large amount of solute atoms (e.g. Mn, Ni, Ti, Al and Mo) were added, the strength of alloys in the SHT state was increased when compared to that of the base steel. The strengthening achieved by substitutional solute atoms is, in general, greater the larger the difference in atomic size of the solute from that of iron. On the other hand, the differences in the elastic behaviour of solute and solvent atoms are also important in determining the overall solid solution strengthening. $\mathrm{Mn}$ is an effective strengthener, but other elements, such as Mo and Al, have more significant effect [197]. Therefore, increasing Mn content did not result in a significant increase in the lattice distortions (Table 7-2). Hence, there is no evident difference of the solid-solution strengthening among the three alloys. While after aging for a long time, owing to the precipitation and the formation of reverted austenite which consumed a large number of solute atoms, the supersaturated $\alpha^{\prime}$-martensite matrix gradually restored (Table 7-2). This indicates that the solid-solution strengthening after long-term aging was less important than that in the SHT and earlier aging state.

Table 7-2 Lattice parameter $(\AA)$ of $\alpha^{\prime}$-martensite in the three alloy in different heat treatment conditions.

\begin{tabular}{rcc}
\hline & SHT & $500^{\circ} \mathrm{C} / 10080 \mathrm{~min}$ \\
\hline $7 \% \mathrm{Mn}$ & 2.8820 & 2.8753 \\
$10 \% \mathrm{Mn}$ & 2.8863 & 2.8776 \\
$12 \% \mathrm{Mn}$ & 2.8878 & 2.8789 \\
\hline
\end{tabular}

EBSD maps of the 7\%, 10\% and 12\% Mn alloys in the SHT state (Figure 4-2) have demonstrated that increasing the Mn content of alloy significantly refined both the prior austenite grain size and packet size of lath martensite. Therefore, finer prior austenite grains and martensite packets were observed in the $12 \% \mathrm{Mn}$ alloy in the SHT state compared to other alloys. Despite this, the tensile curves in Figure 4-9 reveal that the differences of YS, UTS and TE among the three alloys are small. It is 
proposed that this is because the solid-solution strengthening prevailed in the SHT state. Another reason is the grain boundaries of martensite packets are too weak to resist dislocation. As experimental evidence reveals that the effective grain sizes had a good resistance against coarsening during aging owing to the segregation of $\mathrm{Mn}$ and the formation of reverted austenite (Figure 7-1), the grain refinement strengthening in the aged conditions was considered to be the same as in the SHT state.
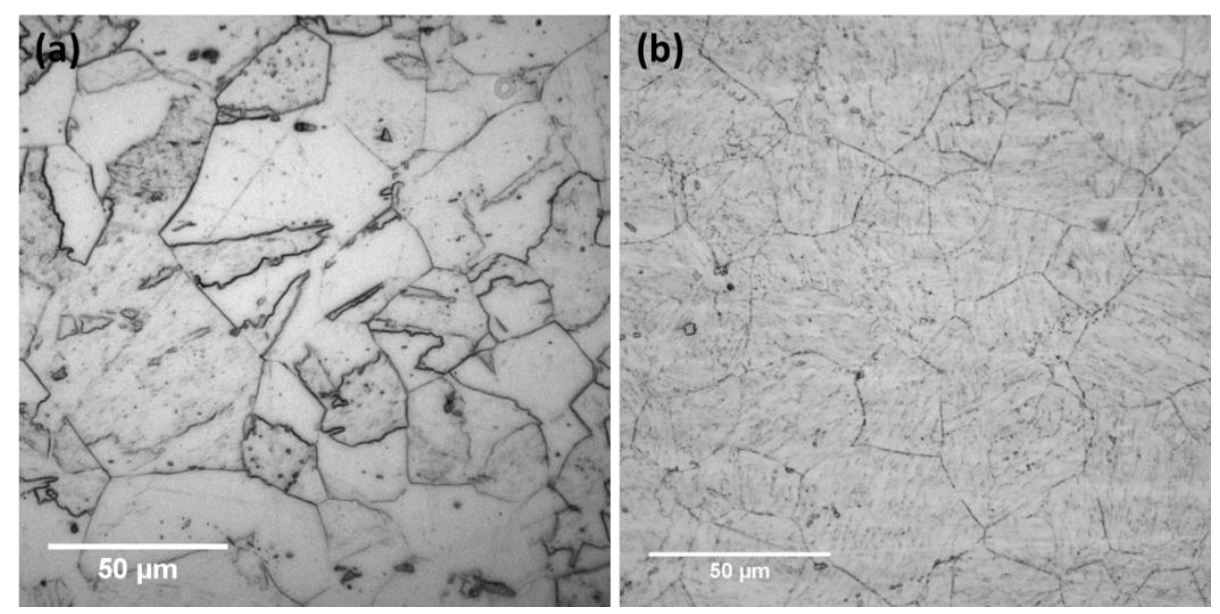

Figure 7-1 The optical micrographs of 7\% Mn alloy (a) at SHT state and (b) $500{ }^{\circ} \mathrm{C} / 2880$ min state.

\subsection{Precipitation strengthening mechanism}

For precipitation-strengthened alloys, if the sum of $\sigma_{B}+\sigma_{S S}+\sigma_{G R}+\sigma_{T R}$ is defined as $\sigma_{0}$, the strengthening can be accounted for an Orowan mechanism [48]:

$$
\sigma_{y}=\sigma_{0}+\frac{T}{b \Lambda}
$$

where $T$ is the line tension, $b$ is the Burgers vector and $\Lambda$ is the interparticle spacing. After examining Orowan theory in detail, Ansell and his colleagues [256][257] proposed a modified form of the Orowan equation in which $\sigma_{y}$ is expressed as:

$$
\sigma_{y}=\sigma_{0}+\frac{2 G b \emptyset \ln (\Lambda-d / 2 b)}{4 \pi(\Lambda-d)}
$$


where $G$ is the shear modulus of matrix, $\varnothing$ is related to the Poisson's ratio of matrix by $\varnothing=\left[1+\frac{1}{1-v}\right] / 2$ and $d$ is the mean size of precipitates. In the present study, the peak hardness condition is considered to be strengthened mainly by the precipitation effects. However, in order to avoid the intergranular brittleness, a slightly overaged sample (12\% Mn alloy, $500{ }^{\circ} \mathrm{C} / 240 \mathrm{~min}$ state) was studied to estimate the precipitation strengthening mechanism (see Figure 7-2(a)). Since this sample had a small amount of reverted austenite (below 3 vol.\%), the effect of reverted austenite on strengthening would have been small. The value of $\sigma_{0}$ in peak hardness condition is assumed to be equal to that of $\sigma_{y}$ in the SHT state. The average precipitate size of this sample is $7.4 \mathrm{~nm}$ (Table 5-1) and the interparticle spacing was $\sim 40 \mathrm{~nm}$. Taking $G=71 \mathrm{GPa}$ [33], $|b|=0.249 \mathrm{~nm}$ [48], $v=0.3$ [54] and $\sigma_{0}=820 \mathrm{MPa}$, the calculated $\sigma_{y}$ is $1258 \mathrm{MPa}$ which is in good agreement with the experimental value $(\sim 1250 \mathrm{MPa})$. Moreover, this does suggest that the small amount of reverted austenite in this sample did not contribute greatly to strength.

Based on the Orowan mechanism, it is not surprising to see a decrease in the YS during the overaged period. However, the experimental $\sigma_{y}$ in the $500{ }^{\circ} \mathrm{C} / 5760 \mathrm{~min}$ state (882 MPa) is much lower than the value calculated by Equation (7.3) (1090 $\mathrm{MPa}$ ) and is almost at the same level with the YS in the SHT state (834 MPa). This discrepancy is attributed to two factors: (i) the precipitation and austenite reversion for the longer aging times consumed a large amount of solute atoms, which weakened the solid-solution strengthening of the $\alpha^{\prime}$-martensite matrix; (ii) the annihilation of dislocation slips as a result of the recovery of $\alpha^{\prime}$-martensite matrix after long-term aging will have reduced its strength. The softening of $\alpha^{\prime}$ martensite matrix led to the fact that the hardness of $\alpha^{\prime}$ martensite matrix was even lower than that of reverted austenite in the overaged stage.

Based on the above discussion, the precipitation strengthening mechanism is summarized as follows. At the early stage of aging, strengthening is associated with the stress required for dislocations to cut through coherent $\mathrm{L} 2{ }_{1}$-ordered $\mathrm{Ni}_{2} \mathrm{TiAl}$ precipitates. The stress to cut through the precipitate is assumed to be governed by the coherency stresses and internal ordering of precipitates [33]. With the growth of the precipitates, the stress required for dislocation to cut through them increases and 
so the yield strength increases. Unfortunately, it was not possible to investigate the strengthening behaviour at this stage due to the intergranular brittleness in this study. Further increasing in the precipitate size led to the initiation of Orowan mechanism. According to Orowan mechanism, the coarsening of precipitates featured with the increase of interparticle spacing after long-term aging result in the decrease of strength.
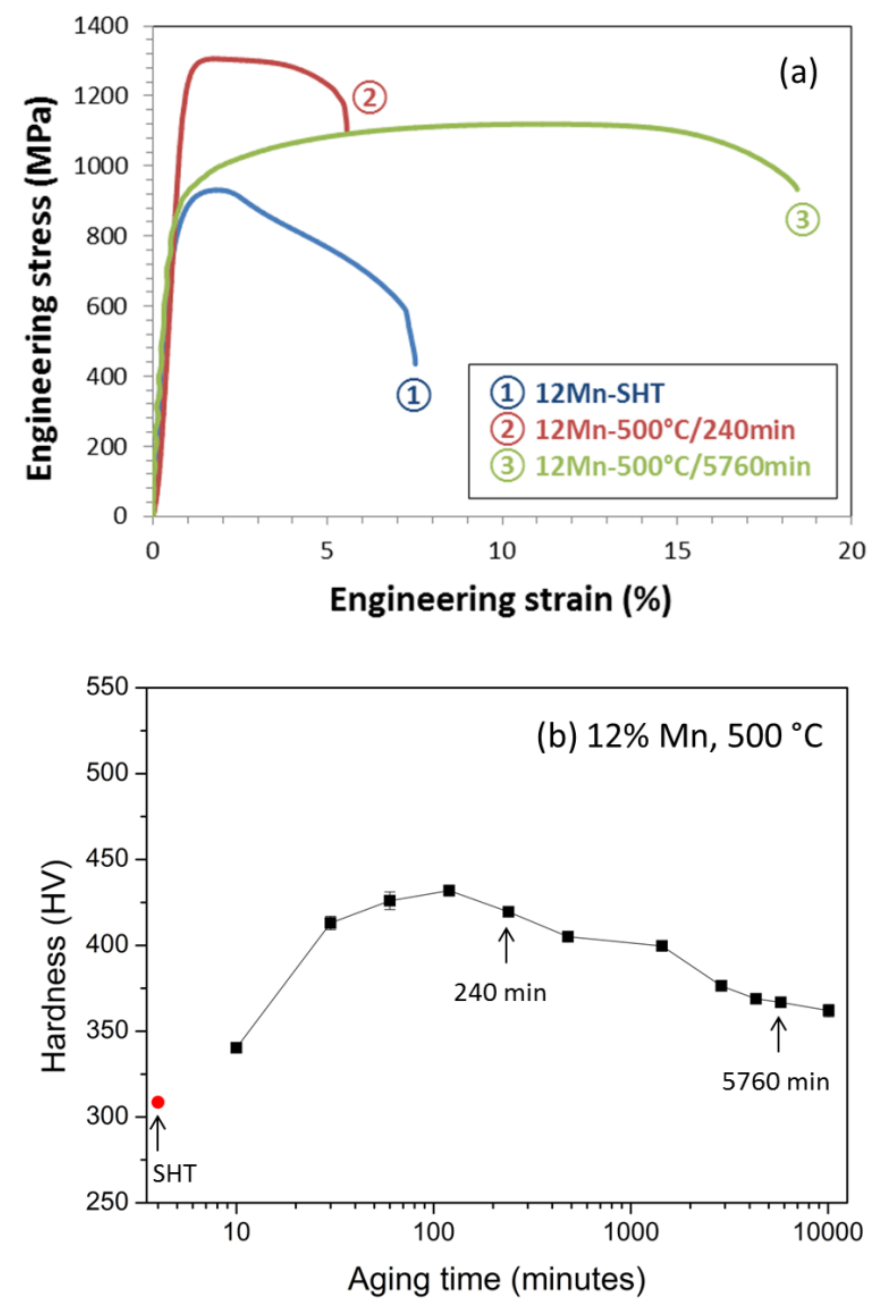

Figure 7-2 (a) The engineering stress-strain curves of 12\% Mn alloy in different heat treatment conditions; (b) the corresponding hardness evolution of $12 \% \mathrm{Mn}$ alloy aged at $500{ }^{\circ} \mathrm{C}$. 


\subsection{Austenite engineering in overaged stage}

There are three essential plastic deformation mechanisms in austenite at room temperature: dislocation slip, deformation-induced martensitic transformation and mechanical twinning [26]. In this section, an attempt will be made to understand which deformation modes occurred in these Mn-based maraging steels. This is significant if further improvement of the mechanical properties is required, as both theoretical and experimental studies have demonstrated that invoking the desired operation modes of plastic deformation in austenite can avoid the loss of ductility shortly after yielding [26].

Figure 7-3 displays the microstructural observation and XRD quantitative analysis of the over-aged $10 \%$ and $12 \% \mathrm{Mn}$ alloys $\left(500{ }^{\circ} \mathrm{C} / 5760 \mathrm{~min}\right.$ state) after tensile testing. The plastic deformation did not lead to a significant decrease in the amount of austenite. A more evident decrease in the volume fraction of austenite after tensile testing was observed in the $10 \% \mathrm{Mn}$ alloy, which might be associated with a lower stability of the austenite due to lower Mn concentration. All the evidence indicates that the reverted austenite possessed such a high stability against deformationinduced martensitic transformation that the TRIP effect was not a strong factor in the two alloys. This conclusion is in agreement with our previous nanoindentation study (Section 6.5) in which the TRIP effect was seldom observed during indentation. 

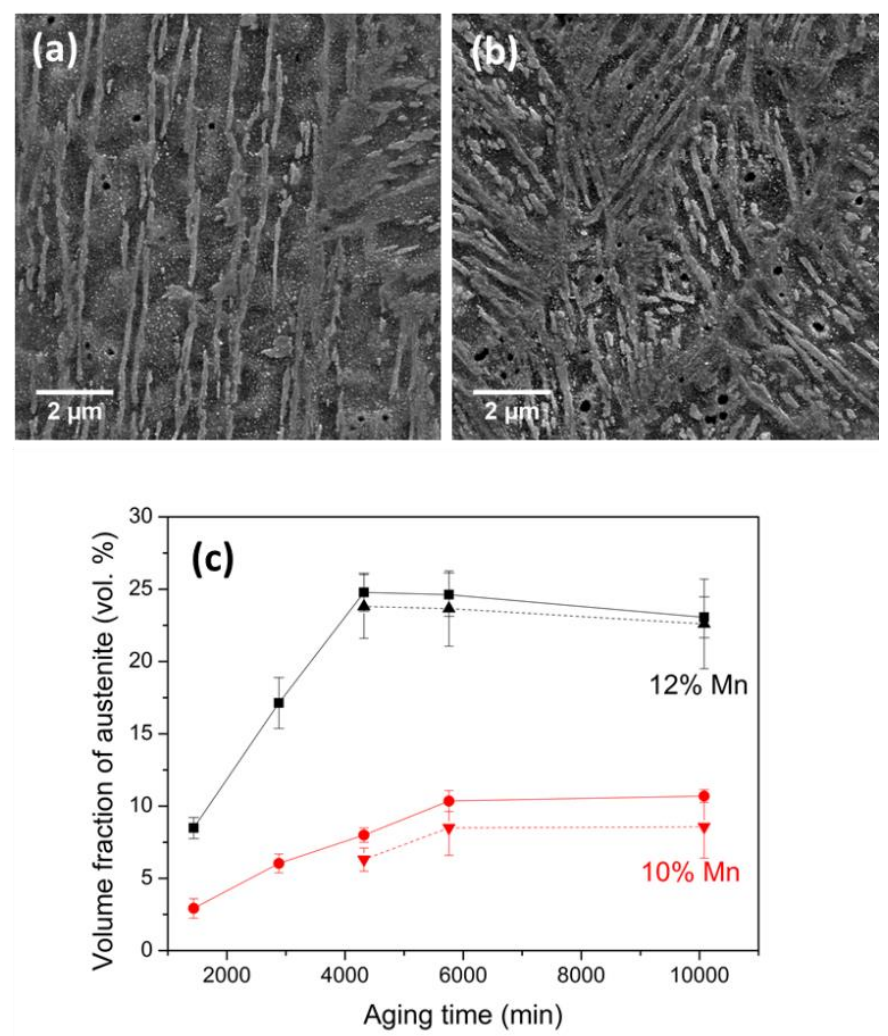

Figure 7-3 SEM images of samples after-tensile (a) $10 \% \mathrm{Mn}$ and (b) $12 \% \mathrm{Mn}$ alloys aged at $500{ }^{\circ} \mathrm{C}$ for $5760 \mathrm{~min}$. (c) The volume fractions (vol.\%) of reverted austenite as a function of aging time at the temperature of $500{ }^{\circ} \mathrm{C}$ (solid lines: before tensile testing; dash lines: after tensile testing).

\subsubsection{The effect of reverted austenite on strengthening}

As shown in Figure 4-8, when aging at $500{ }^{\circ} \mathrm{C}$, the hardness of the 10 and $12 \% \mathrm{Mn}$ alloys moderately decreased during the overaged stage. On the other hand, secondary hardening was observed at this stage in both alloys, which suggested the possible formation of an additional phase. Experimental evidence showed that it was associated with the austenite reversion in both alloys. As Figure 7-4(a) shows, it is clear that the formation of austenite (can be detected by XRD (> 3 vol. \%)) in the $10 \%$ Mn alloy started from aging for $1440 \mathrm{~min}$, which coincided with the onset of secondary hardening. Although the austenite nanolayers were observed earlier before the $500^{\circ} \mathrm{C} / 1440 \mathrm{~min}$ state, e.g. austenite layers along the prior austenite grain boundaries at the early aging stage in Figure 6-1, it is not believed that the plastic deformation mechanisms could occurred in the austenite nanolayers. A similar 
coincidence of detecting reverted austenite formation and secondary hardening also existed in the $12 \% \mathrm{Mn}$ alloys aged at $500{ }^{\circ} \mathrm{C}$ (Figure 7-4(b)).

In order to discuss the work hardening behaviour, true stress-strain curves are introduced in this section. Figure 7-5(a) displays the representative true stress-strain curves of $12 \% \mathrm{Mn}$ alloy when aged at $500{ }^{\circ} \mathrm{C}$ for $480 \mathrm{~min}$ and $5760 \mathrm{~min}$. True stress-strain curves of the $12 \% \mathrm{Mn}$ alloy in the SHT state and 10\% Mn alloy at $500{ }^{\circ} \mathrm{C} / 5760 \mathrm{~min}$ state are also displayed in Figure 7-5(a) for a comparison. The following discussion about the tensile properties in this section is all based on the true stress-strain data.
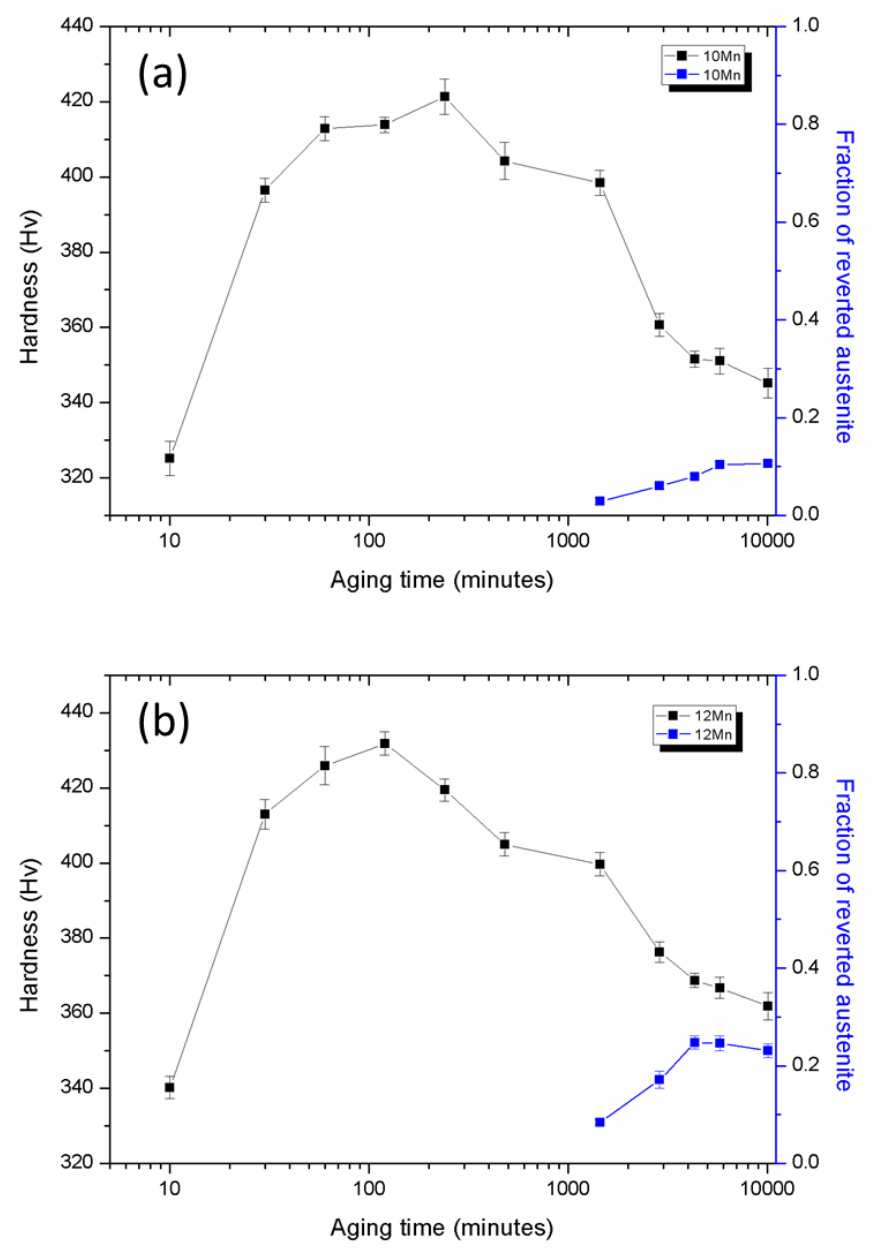

Figure 7-4 Hardness evolution vs. volume fraction of austenite as a function of time in (a) $10 \%$ $\mathrm{Mn}$ and (b) $12 \% \mathrm{Mn}$ alloys when aged at $500{ }^{\circ} \mathrm{C}$. 
As shown in Figure 7-5, the $12 \% \mathrm{Mn}$ alloy in the $500{ }^{\circ} \mathrm{C} / 480 \mathrm{~min}$ state exhibited an impressive yield stress of $\sim 1320 \mathrm{MPa}$ but failed shortly after yielding with a maximum true strain of $5.4 \%$. The high yield stress was attributed to the precipitation strengthening mechanism which, however, could not provide sufficient work hardening after yielding, although the work hardening was still evident when compared to that of the SHT state. In contrast, the $12 \% \mathrm{Mn}$ alloy in the $500{ }^{\circ} \mathrm{C} /$ 5760 min state exhibited steady work hardening after reaching the yield stress of $900 \mathrm{MPa}$. This subsequent work hardening greatly enhanced both the strength (up to $1490 \mathrm{MPa})$ and ductility ( 16.9\%). A significant but lower level of work hardening (maximum true stress: $1366 \mathrm{MPa}$, maximum true strain: $15.7 \%$ ) existed in the $10 \%$ $\mathrm{Mn}$ alloy in the $500{ }^{\circ} \mathrm{C} / 5760 \mathrm{~min}$ state (Figure 7-5). This work hardening apparently compensated the loss of yield stress after long-term aging, and more importantly, it was accompanied by an increase of ductility. As it has been shown that there was limited transformation of the reverted austenite to martensite during the deformation of both alloys, it is not likely that the work hardening could be primarily attributed to the TRIP effect. Given that the twinned structure of austenite was not observed in the two samples either, it is proposed that the deformation mechanism of reverted austenite was dominated by the dislocation slip with a small amount of the TRIP effect. This is quite possible when considering the high stability of the reverted austenite in the two alloys aged at $500{ }^{\circ} \mathrm{C}$. 


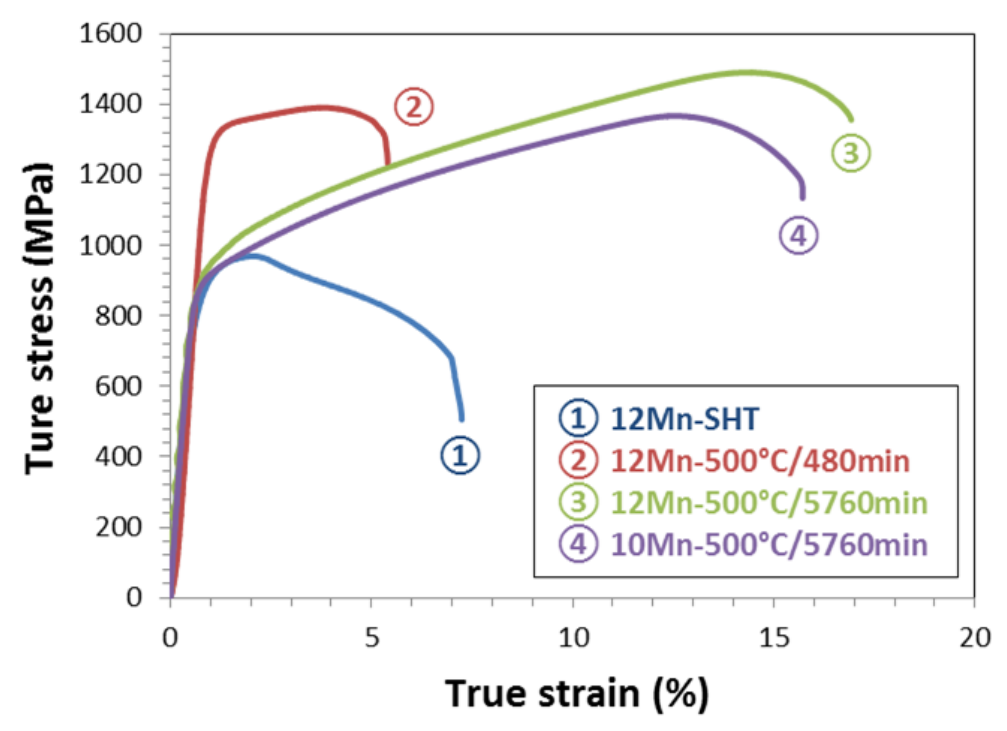

Figure 7-5 (a) The true stress-strain curves of the $12 \% \mathrm{Mn}$ alloy in different heat treatment conditions; the true stress-strain curves of the $10 \% \mathrm{Mn}$ alloy in the $500{ }^{\circ} \mathrm{C} / 5760 \mathrm{~min}$ state is also displayed for comparison.

It is known that $\alpha^{\prime}$-martensite tends to nucleate at grain boundaries by a straincontrolled mechanism, or in some cases by stress-assisted nucleation process. Yen et al. [26] pointed out that when the grain size of austenite is small, it is difficult to form the multiple-variant fault structure which serve as the dominant sites for the nucleation of strain-induced martensite and therefore the stress-assisted martensite nucleation would became the predominant mechanism in this situation. In this work, a lot of evidence has confirmed that the reverted austenite formed in Mn-based maraging steels aged at $500{ }^{\circ} \mathrm{C}$ was very stable. Deformation-induced transformation to martensite did not occur by a large scale even when subjected to a true strain up to $16.9 \%$. As the deformation behaviours of nano-scale austenite in this work coincided with the factors required for the stress-assisted nucleation mechanism in Ref. [26], it is proposed that the limited TRIP effect in this work was the result of a stressassisted martensitic transformation process rather than the well-known straininduced martensitic transformation.

The thermodynamic modelling has demonstrated that the stress-assisted mechanism for the deformation-induced $\alpha^{\prime}$-martensite transformation is very sensitive to the 
chemical composition of austenite, particularly the Mn concentration [26]. Based on the principles of stress-assisted martensitic transformation, Yen et al. [26] suggested that the compositional differences in austenite could lead to a remarkable difference in the efficiency of TRIP effect. It has been demonstrated by experiment that an additional stress of $587 \mathrm{MPa}$ is required to trigger the deformation-induced martensitic transformation in austenite with $2.6 \mathrm{wt}$.\% higher of Mn concentration. Therefore, reverted austenite with relatively high Mn concentrations (in the range of 20-26.1 wt.\%) in this study is believed to require a correspondingly high stress to induce the martensitic transformation. In reality, most austenite grains in Mn-based maraging steels reached the plastic instability before the stress threshold required for martensitic transformation was reached. Only a small amount of austenite grains with lower Mn concentrations had the martensitic transformation during deformation.

\subsubsection{The effect of reverted austenite on ductility}

In contrast to some literature suggesting that the brittleness was associated with the formation of reverted austenite [113], the present work suggests that the ductility is significantly improved by the formation of ductile austenite at grain boundaries.

Basically, the embrittlement is caused by two factors, first, the segregation of impurity or alloying elements along grain boundaries and second, preferential precipitation of second phase(s) at grain boundaries. As discussed in Section 2.2.4, researches presented numerous experimental results to support both mechanisms in Fe-Mn alloys [92][95][113][116][117][118][119][121]. The main difficulty in studying the embrittlement phenomenon is the observation of atomic scale segregation at boundaries. Initially, the results were only speculative. Later, Auger electron spectroscopy (AES) was applied to elucidate possible segregation at grain boundaries and these investigations indeed identified the segregation of $\mathrm{Mn}$ to boundaries [49][121][122]. In this study, the Auger electron spectroscopy was not performed to study the elemental segregation at the interface. However, highresolution TEM-EDS analysis has confirmed the presence of Mn-rich nanolayer at grain boundaries in the early aging stage (Section 6.3). 
The tensile results in conjunction with the fractographs of aged 10 and $12 \% \mathrm{Mn}$ alloys revealed that the aging treatment resulted in the intergranular brittleness in the initial stage of aging. While after longer aging durations, or higher aging temperatures or increasing $\mathrm{Mn}$ content led to a transition in the fracture mode to transgranular dimpled ductile fracture. These factors have been demonstrated to benefit the formation of reverted austenite as well. For example, 12\% Mn alloy aged at $500{ }^{\circ} \mathrm{C}$ exhibited intergranular embrittlement in the initial aging stage until the ductility suddenly increased to $5.5 \%$ after aging for $240 \mathrm{~min}$. At this time, the volume fraction of austenite could not be detected by XRD, only thin austenite nanolayers were visible under SEM and TEM observations. As the increasing of aging time, the ductility gradually increased to $17.8 \%$ in the $500{ }^{\circ} \mathrm{C} / 2880 \mathrm{~min}$ state with the austenite fraction of $17.2 \%$. The whole transformation process can be described as: in the initial aging stage, the segregation of $\mathrm{Mn}$ at the grain boundaries increased the DBTT and thus gave rise to the intergranular embrittlement under external load. Although the subsequent formation of austenite nanolayers removed the intergranular embrittlement, the ductility was still unsatisfactory. Thanks to the growth of austenite layers via accumulated Mn segregation, the presence of ductile lath-like austenite significantly improved the ductility of these alloys. On the other hand, once the evident ductility was achieved, the further increase of austenite fraction did not lead to a corresponding improvement in ductility (Figure 4-11 and Figure 4-12). This finding once again confirmed that the TRIP effect was not likely to be extensive in these alloys, as the TRIP effect of austenite is known to improve the ductility and it is supposed that there is an approximately linear relationship between the volume fraction of austenite and the increment of ductility in the case of TRIP steels [15].

\subsection{Summary}

Studies on newly-developed materials mainly focus on optimizing the microstructure by tailoring the heat treatment parameters and chemical composition with the aim of obtaining the desired properties. Specifically in this work, the Mn content of the alloy, aging temperature and duration are the most important factors. Based on the 
above discussion, the $12 \% \mathrm{Mn}$ alloy, which had a relatively smaller grain size and higher amount of reverted austenite after aging, had many advantages. Further increasing the Mn content beyond 12 wt.\%, however, would lead to the TWIP austenitic steels [18]. In terms of aging conditions, the effort to attain a good balance between the aging temperature and duration has been made. The $12 \% \mathrm{Mn}$ alloy in the $500{ }^{\circ} \mathrm{C} / 480 \mathrm{~min}$ state exhibits a high yield stress ( 1320 MPa) which is attributed to the interaction between dense precipitates and dislocations in the martensite matrix, whereas the poor ductility (5.4\%) and limited work hardening is due to the small amount of reverted austenite nanolayers which cannot provide plastic deformation. In contrast, the lower yield stress ( $900 \mathrm{MPa})$ of the $12 \% \mathrm{Mn}$ alloy in the $500{ }^{\circ} \mathrm{C} / 5760 \mathrm{~min}$ state is the result of coarse precipitates and martensite softening after long-term aging. But owing to the considerable amount of lath-like reverted austenite formed at grain boundaries, the alloys exhibited a good work hardening leading to a maximum stress of $1490 \mathrm{MPa}$ after yielding. Therefore, there is a contradiction between the requirement for short aging time to avoid the coarsening of precipitates and the softening of martensite matrix and the requirement for long-term aging to form a certain amount of lath-like austenite. Previous studies have revealed that the precipitation kinetics of $\mathrm{Ni}_{2} \mathrm{TiAl}$ was very fast due to the rapid diffusivity of participating elements at $500{ }^{\circ} \mathrm{C}$, whereas the formation of lath-like austenite was limited by the diffusion of Mn. The analyses on the formation of precipitates and reverted austenite have demonstrated that the kinetics of both processes were strongly associated with the aging temperature. Elevating aging temperature accelerated both processes, but some evidence indicates that the influence of aging temperature on the austenite formation was more prominent than that on the precipitation. For example, decreasing the aging temperature to $420{ }^{\circ} \mathrm{C}$ led to the volume fraction reducing to almost zero even after aged for $10080 \mathrm{~min}$, whereas a number of precipitates with a size of $\sim 5.5 \mathrm{~nm}$ could be observed in the $12 \%$ Mn alloy aged at $420{ }^{\circ} \mathrm{C}$ for 10080 min (Figure 5-2). Based on this discussion, the idea of aging at a higher temperature for a shorter duration is proposed. Most importantly, it is suggested by the MatCalc calculation that higher aging temperature corresponds to a lower Mn concentration of austenite. As it has been demonstrated that the stability of austenite is closely dependent on the concentration of austenite 
stabilizing elements, the increase of aging temperature is believed to lead to a less stable austenite in which deformation-induced martensitic transformation is possible. In this case, a further improvement of both strength and ductility due to TRIP effect can be expected in these alloys.

Figure 7-6 shows the SEM images of the $12 \%$ Mn alloy aged at $540{ }^{\circ} \mathrm{C}$ for $360 \mathrm{~min}$. It is clear that the reverted austenite had been formed at grain boundaries in this stage (Figure 7-6(a)). Besides, a large number of fine precipitates were observed as well (Figure 7-6(b)). In the future study, the tensile testing and nanoindentation will be carried out on this sample to examine its mechanical properties.
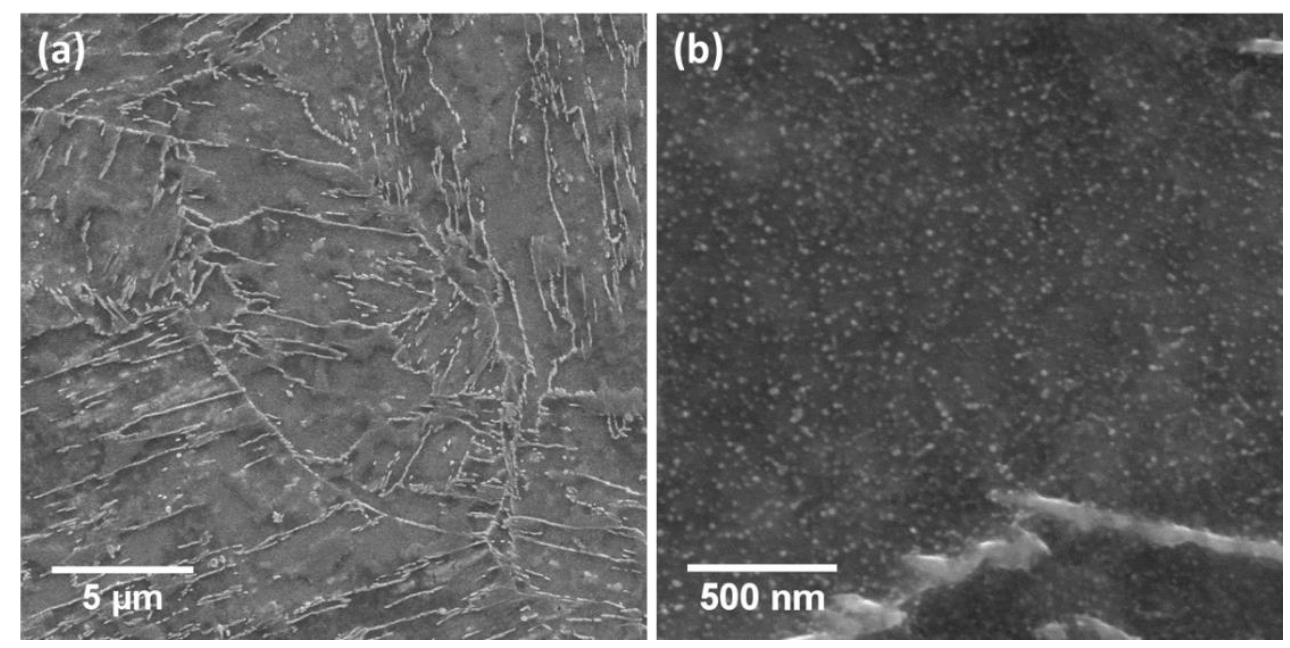

Figure 7-6 SEM images of $12 \% \mathrm{Mn}$ alloy aged at $540{ }^{\circ} \mathrm{C}$ for $360 \mathrm{~min}$ : (a) reverted austenite formed along grain boundaries and (b) nano-precipitates. 


\section{Chapter 8 Conclusions and future work}

\subsection{Phase identification}

Thermodynamic calculations by MatCalc indicated that the dominant equilibrium phases of Mn-based maraging steels at the aging temperatures $420-500{ }^{\circ} \mathrm{C}$ are ferrite and austenite. But it did not predict the existence of $\mathrm{Ni}_{2} \mathrm{TiAl}$ intermetallic phase. Instead, a laves phase $\mathrm{Fe}_{3} \mathrm{Ti}(\mathrm{Mo})$ is considered to be the precipitate phase according to the calculation. This discrepancy implies that an improvement to complete the thermodynamic database is required. Another reason may be that the real microconstituent is far from the equilibrium and is difficult to comprehensively predict. The situation is further complicated when considering the variety of alloying elements in this alloying system and the substantial variations in heat treatment paths. Phase identification by XRD confirmed the formation of reverted austenite during aging at $420-500{ }^{\circ} \mathrm{C}$. The amount of reverted austenite increased with the aging temperature and duration. Higher $\mathrm{Mn}$ content of the alloy also promoted the austenite reversion. In addition, XRD analyses revealed the existence of $\varepsilon$-martensite in the over-aged samples, but no evidence of precipitates was found in XRD patterns.

\subsection{Hardness and tensile results}

In the SHT state, the addition of Mn has been demonstrated to refine both the prior austenite grains and martensite packets via reducing the $A_{e 3}$ and $M_{s}$ temperatures. This grain refinement is believed to contribute to the higher hardness of $12 \% \mathrm{Mn}$ alloy in the SHT state.

Aging at $460{ }^{\circ} \mathrm{C}$ and $500{ }^{\circ} \mathrm{C}$ exhibited a typical hardness evolution of the precipitation-strengthened alloys. Increasing the aging temperature led to a more rapid hardening towards the peak hardness followed by a moderate decrease of hardness in the over-aged regime. Slower hardening kinetics but more significant hardening effect existed at $420{ }^{\circ} \mathrm{C}$. In terms of the effect of Mn content, a quicker 
response to thermal treatment and more significant hardening was found in the $12 \%$ Mn alloy.

Embrittlement occurred in samples aged at lower temperatures or for shorter times. Increasing the aging temperature and duration significantly improved the embrittlement phenomena. Increasing the Mn content of the alloy also benefited the ductility. The fracture mode of the solution-treated samples was a mix of cleavage brittle fracture and dimple ductile fracture. Intergranular brittle fracture was observed in the early aging stage. The embrittlement phenomenon was much improved after prolonged aging process and the fracture mode gradually transformed to a transgranular dimpled ductile fracture. Both increasing Mn content and aging temperature accelerated the improvement in the ductility. The $12 \% \mathrm{Mn}$ alloy aged at $500{ }^{\circ} \mathrm{C}$ for $5760 \mathrm{~min}$ exhibited a UTS of $1120 \mathrm{MPa}$ with TE of $18.4 \%$.

\subsection{Precipitation behaviour of $\mathrm{L}_{1}$-ordered $\mathrm{Ni}_{2} \mathrm{TiAl}$ phase}

The compositional analyses identified that the nano-scale precipitates in the Mnbased maraging steels are a non-stoichiometric $\mathrm{Ni}_{2} \mathrm{TiAl}$ intermetallic phase with minor Fe and Mn substitution. This non-stoichiometric composition is considered to enhance the ductility of the $\mathrm{Ni}_{2} \mathrm{TiAl}$ phase.

HREM in conjunction with SAED study revealed the crystal structure of $\mathrm{Ni}_{2} \mathrm{TiAl}$ phase is $\mathrm{L} 2{ }_{1}$-ordered which is expected to have a better creep resistance than B2$\mathrm{NiAl}$ phase. The orientation relationship between the $\mathrm{L}_{1}$-ordered $\mathrm{Ni}_{2} \mathrm{TiAl}$ phase and $b c c$ martensite matrix was: $(220)_{L 2_{1}}\left\|(110)_{b c c},[001]_{L 2_{1}}\right\|[001]_{b c c}$; $(220)_{L 2_{1}}\left\|(110)_{b c c},[011]_{L 2_{1}}\right\|[011]_{b c c} ;(220)_{L 2_{1}}\left\|(110)_{b c c},[\overline{1} 11]_{L 2_{1}}\right\|[\overline{1} 11]_{b c c}$. One eighth of the crystal structure of the $\mathrm{L} 2_{1}-\mathrm{Ni}_{2} \mathrm{TiAl}$ phase is a bcc cubic lattice which has a similar lattice parameter with the bcc matrix. The lattice misfit between them is calculated as $1.24 \%$. This small lattice misfit together with the special orientation relationship indicates that the $\mathrm{L} 2{ }_{1}$-ordered $\mathrm{Ni}_{2} \mathrm{TiAl}$ precipitates are not only coherent but also coplanar with the $\alpha^{\prime}$-martensite matrix. The coherent relationship is confirmed by the bright-field TEM observation which exhibited 
classic dumb-bell dynamical diffraction contrast from the precipitates under twobeam conditions.

Various microstructural observations, such as by bright-field TEM, HREM and HAADF, suggested the existence of core/shell structure in the precipitates. So far, the exact nature of the heterogeneity is still not clear. The current evidence indicates that the two-phase $\mathrm{L} 2{ }_{1}-\mathrm{Ni}_{2} \mathrm{TiAl} / \mathrm{B} 2-\mathrm{NiAl}$ precipitates might be possible.

Experimental results revealed that the coarsening rate constants of precipitates in Mn-based maraging steels obey the diffusion-controlled coarsening kinetic form $\bar{r}^{3} \sim K t$ predicted by LSW theory (even though the alloy clearly broke the assumption of the LSW theory of a zero volume fraction of precipitates). On the other hand, the experimental particle size distributions (PSDs) were much broader than the theoretical PSD function, as the PSDs are believed to be more sensitive to the volume fraction than the coarsening rate constants.

Both thermodynamics and kinetics analyses supported the view that the Mn atoms participated in the nucleation of precipitation and reduced the misfit strain energy at the interface. Hence, higher Mn content is considered to promote the nucleation and increase the number density of precipitates, which has been confirmed by the microstructural observations.

\subsection{Reverted austenite and its mechanical stability}

The formation of reverted austenite nanolayers at grain boundaries initiated at the onset of aging by a diffusionless shear mechanism since the critical $\mathrm{Mn}$ concentration for austenite reversion at the interface was very low. The nucleation of reverted austenite within martensite laths was kinetically slower due to a lower driving force for phase transformation. In the following aging process, the accumulated Mn segregation to the new martensite/austenite grain boundaries led to the austenite nanolayers growing to lath-like reverted austenite, which means the lateral growth of austenite was supported by the diffusion of Mn. As the diffusion rate of $\mathrm{Mn}$ in the martensite matrix was relatively low, the diffusion-controlled austenite growth was fairly slow. In addition, the thermodynamic resistance to 
coalescence between adjacent reverted austenite grains is another reason why the width of reverted austenite could maintain in the range 70-200 nm for a long time.

The width of reverted austenite was not closely associated with the nominal Mn content of the alloy, but it is found that it slightly increased with the increase of aging temperature owing to the higher driving force for $\mathrm{Mn}$ diffusion at higher temperature.

The experimental chemical composition profile of reverted austenite was partially consistent with that given by the Fe-Mn phase diagram. The segregation of Ti and Mo on grain boundaries in the initial aging stage resulted in the Mn concentration of austenite nanolayers being far from that indicated by the equilibrium phase diagram. The high concentration of $\mathrm{Ti}$ and Mo gradually vanished with the enrichment of $\mathrm{Mn}$ during the succeeding aging process. Therefore, the chemical composition of ferrite and lath-like austenite exhibited good agreement with the phase diagram. The TEMEDS analyses revealed the Mn concentration of lath-like austenite was at the level of $\sim 24$ at.\% which is higher than that of retained austenite (8-12 at.\%) reported in conventional Mn-based TRIP or Q\&P steels [27][154][34][50].

The volume fraction of reverted austenite increased almost linearly with the logarithm of aging time until it was saturated. The experimental results of the maximum austenite fractions (e.g. $\sim 10$ vol. $\%$ for $10 \% \mathrm{Mn}$ alloy aged at $500{ }^{\circ} \mathrm{C}$ and $\sim 24$ vol.\% for $12 \% \mathrm{Mn}$ alloy aged at $500{ }^{\circ} \mathrm{C}$ ) were significantly lower than the values predicted by the Fe-Mn phase diagram (36.0 vol.\% for $\mathrm{Fe}-10 \mathrm{Mn}$ at $500{ }^{\circ} \mathrm{C}$ and $\sim 47.5$ vol. $\%$ for $\mathrm{Fe}-12 \mathrm{Mn}$ at $500{ }^{\circ} \mathrm{C}$ ). This is mainly attributed to the fact that an amount of reverted austenite transformed to $\varepsilon$-martensite during cooling. The addition of $\mathrm{Ti}$ and $\mathrm{Al}$ may also be a reason for the discrepancy.

Most of the reverted austenite in the Mn-based maraging steels exhibited a K-S or N orientation relationships with the residual martensite. It is believed that both the K-S and $\mathrm{N}$ orientation relationships contributed to the mechanical stability of reverted austenite. However, nanoindentation results revealed that the high stability of reverted austenite in Mn-based maraging steels was mainly attributed to the high $\mathrm{Mn}$ concentration of austenite. The nano-size of reverted austenite was also considered to 
be responsible for the high stability, but more evidence is needed to support this viewpoint.

\subsection{Optimal heat treatment suggestions}

Based on the above discussion, the $12 \% \mathrm{Mn}$ alloy, which had a relatively smaller grain size together with a higher potential for austenite reversion, has been demonstrated to possess advantages for the automotive applications. In terms of the aging conditions, the optimum aging temperature for Mn-based maraging steels is $460{ }^{\circ} \mathrm{C}$ if the balance between the strengthening effect and production efficiency is considered, but in reality the serious embrittlement that occurs at this temperature would limit its use. Therefore, the aging temperature primarily studied in this work was $500{ }^{\circ} \mathrm{C}$. The $12 \% \mathrm{Mn}$ alloy aged at $500{ }^{\circ} \mathrm{C} / 480 \mathrm{~min}$ state exhibited a high yield stress (true stress: $\sim 1320 \mathrm{MPa}$ ) but poor ductility (true strain: 5.4\%), which resulted from the interaction between dense precipitates and dislocations. In contrast, the $12 \%$ Mn alloy aged at $500{ }^{\circ} \mathrm{C} / 5760$ min state exhibited a lower yield stress (true stress: 900 MPa) but good work hardening leading to a UTS of $1490 \mathrm{MPa}$ (true stress), which was attributed to the formation of reverted austenite. Therefore, there is a contradiction between the requirement for a short aging duration to avoid the softening of martensite matrix (including the coarsening of precipitates) and the requirement for a long-term aging to form lath-like reverted austenite. Experimental studies revealed that the kinetics of both the precipitation and austenite reversion were strongly dependent on the aging temperature, but the influence of aging temperature on austenite formation was more prominent than that on precipitation. Therefore, it is proposed that aging at higher temperature for shorter duration might be a method to address the conflicting requirements. More importantly, higher aging temperature corresponds to a lower Mn concentration of austenite which made the occurrence of TRIP effect possible. 


\subsection{Future work}

Based on the findings and discussions in this study, the following suggestions for future research are proposed:

1. It has been proposed that the $12 \% \mathrm{Mn}$ alloy aged at higher temperature for a shorter duration to obtain a better combination of strength and ductility. The next step therefore is to define the optimal aging temperature and duration. Dilatometry measurements can be utilized to determine the austenite transformation kinetics instead of by trial and error method. A volume fraction of austenite ( 20 vol.\%) with a mechanical stability which enhances the capacity for work hardening during deformation is the main aim when designing the processing paths.

2. Atom probe analysis which provides the information about the chemical composition, particle size and number density of precipitates at atomic scale, should be used for further characterization of precipitates formed in early aging stage. In addition, APT in combination with HREM can assist the investigation on the core/shell structure.

3. The orientation relationship between the austenite and martensite needs to be further studied by EBSD which can provide better statistics than TEM observations, although this technique will be limited by its resolution.

4. Detailed nanoindentation study on the hardness of each single austenite grain with known Mn concentration and grain size is a direct method to explore the relationship between the mechanical stability and Mn concentration/grain size of austenite phase. Besides, nanoindentation is also an effective technology to estimate the occurrence of martensitic transformation under deformation (a higher maximum force at least above $70 \mu \mathrm{N}$ should be applied to trigger TRIP effect).

5. As $\varepsilon$-martensite in Mn-based maraging steels vanished after tensile testing, investigation on its role in the deformation mechanism is required. 


\section{References}

[1] S. Keeler and M. Kimchi, Eds., Advanced High-Strength Steels Application Guidelines Version 5.0. WorldAutoSteel, 2014.

[2] R. G.Davies, "The deformation behavior of a vanadium-strengthened dual phase steel," Metall. Trans. A, vol. 9, no. 1, pp. 41-52, Jan. 1978.

[3] R. G. Davies, "Influence of martensite composition and content on the properties of dual phase steels," Metall. Trans. A, vol. 9, no. 5, pp. 671-679, May 1978.

[4] N. J. Kim and G. Thomas, "Effects of morphology on the mechanical behavior of a dual phase Fe/2Si/0.1C steel," Metall. Trans. A, vol. 12, no. 3, pp. 483-489, Mar. 1981.

[5] M. Sarwar and R. Priestner, "Influence of ferrite-martensite microstructural morphology on tensile properties of dual-phase steel," J. Mater. Sci., vol. 31, no. 8, pp. 2091-2095, 1996.

[6] G. B. Olson and M. Cohen, "Kinetics of strain-induced martensitic nucleation," Metall. Trans. A, vol. 6, no. 4, pp. 791-795, Apr. 1975.

[7] G. B. Olson and M. Azrin, "Transformation behavior of TRIP steels," Metall. Trans. A, vol. 9, no. 5, pp. 713-721, May 1978.

[8] G. B. Olson and M. Cohen, "Stress-assisted isothermal martensitic transformation: Application to TRIP steels," Metall. Trans. A, vol. 13, no. 11, pp. 1907-1914, Nov. 1982.

[9] S. Vercammen, B. Blanpain, B. C. De Cooman, and P. Wollants, "Cold rolling behaviour of an austenitic $\mathrm{Fe}-30 \mathrm{Mn}-3 \mathrm{Al}-3 \mathrm{Si}$ TWIP-steel: the importance of deformation twinning," Acta Mater., vol. 52, no. 7, pp. 20052012, Apr. 2004.

[10] B. C. De Cooman, O. Kwon, and K.-G. Chin, "State-of-the-knowledge on TWIP steel," Mater. Sci. Technol., vol. 28, no. 5, pp. 513-527, May 2012.

[11] B. C. De Cooman, "Phase transformations in high manganese twinninginduced plasticity (TWIP) steels," in Phase Transformations in Steels, D. V. Edmonds and E. Pereloma, Eds. Woodhead Publishing, 2012.

[12] Y. E. Goldshtein and G. A. Charushnikova, Russ. Metall. Min., vol. 4, p. 72, 1963. 
[13] M. Niikura and J. W. Morris, "Thermal processing of ferritic 5Mn steel for toughness at cryogenic temperatures," Metall. Trans. A, vol. 11, no. 9, pp. 1531-1540, Sep. 1980.

[14] T. Furukawa, "Dependence of strength-ductility characteristics on thermal history in lowcarbon, 5 wt-\%Mn steels," Mater. Sci. Technol., vol. 5, no. 5, p. 465, Jul. 1989.

[15] H. Huang, O. Matsumura, and T. Furukawa, "Retained austenite in low carbon, manganese steel after intercritical heat treatment," Mater. Sci. Technol., vol. 10, no. 7, p. 621, Jul. 1994.

[16] D.-W. Suh, S.-J. Park, T.-H. Lee, C.-S. Oh, and S.-J. Kim, "Influence of Al on the Microstructural Evolution and Mechanical Behavior of Low-Carbon, Manganese Transformation-Induced-Plasticity Steel," Metall. Mater. Trans. A, vol. 41, no. 2, pp. 397-408, Dec. 2009.

[17] J. Shi, X. Sun, M. Wang, W. Hui, H. Dong, and W. Cao, "Enhanced workhardening behavior and mechanical properties in ultrafine-grained steels with large-fractioned metastable austenite," Scr. Mater., vol. 63, no. 8, pp. 815-818, Oct. 2010.

[18] D. Raabe, D. Ponge, O. Dmitrieva, and B. Sander, "Designing Ultrahigh Strength Steels with Good Ductility by Combining Transformation Induced Plasticity and Martensite Aging," Adv. Eng. Mater., vol. 11, no. 7, pp. 547555, Jul. 2009.

[19] U. K. Viswanathan, G. K. Dey, and V. Sethumadhavan, "Effects of austenite reversion during overageing on the mechanical properties of $18 \mathrm{Ni}$ (350) maraging steel," Mater. Sci. Eng. A, vol. 398, no. 1-2, pp. 367-372, 2005.

[20] H. Nakagawa, T. Miyazaki, and H. Yokota, "Effects of aging temperature on the microstructure and mechanical properties of $1.8 \mathrm{Cu}-7.3 \mathrm{Ni}-15.9 \mathrm{Cr}-1.2 \mathrm{Mo}-$ low C, N martensitic precipitation hardening stainless steel," J. Mater. Sci., vol. 35, no. 9, pp. 2245-2253, 2000.

[21] A. Haldar, S. Suwas, and D. Bhattacharjee, Eds., Microstructure and Texture in Steels. London: Springer London, 2009.

[22] B. C. De Cooman, P. Gibbs, S. Lee, and D. K. Matlock, "Transmission Electron Microscopy Analysis of Yielding in Ultrafine-Grained Medium Mn Transformation-Induced Plasticity Steel," Metall. Mater. Trans. A, vol. 44, no. 6, pp. 2563-2572, Feb. 2013.

[23] P. J. Gibbs, E. De Moor, M. J. Merwin, B. Clausen, J. G. Speer, and D. K. Matlock, "Austenite Stability Effects on Tensile Behavior of ManganeseEnriched-Austenite Transformation-Induced Plasticity Steel," Metall. Mater. Trans. A, vol. 42, no. 12, pp. 3691-3702, Apr. 2011. 
[24] R. L. Miller, "Ultrafine-grained microstructures and mechanical properties of alloy steels," Metall. Trans., vol. 3, no. 4, pp. 905-912, Apr. 1972.

[25] J.-M. Jang, S.-J. Kim, N. H. Kang, K.-M. Cho, and D.-W. Suh, "Effects of annealing conditions on microstructure and mechanical properties of low carbon, manganese transformation-induced plasticity steel," Met. Mater. Int., vol. 15, no. 6, pp. 909-916, Dec. 2009.

[26] H.-W. Yen, S. W. Ooi, M. Eizadjou, A. Breen, C.-Y. Huang, H. K. D. H. Bhadeshia, and S. P. Ringer, "Role of stress-assisted martensite in the design of strong ultrafine-grained duplex steels," Acta Mater., vol. 82, pp. 100-114, Jan. 2015.

[27] H. Luo, J. Shi, C. Wang, W. Cao, X. Sun, and H. Dong, "Experimental and numerical analysis on formation of stable austenite during the intercritical annealing of 5Mn steel," Acta Mater., vol. 59, no. 10, pp. 4002-4014, Jun. 2011.

[28] S. Floreen, "The physical metallurgy of maraging steels," Int. Mater. Rev., vol. 13, no. 1, pp. 115-128, 1968.

[29] W. Sha and Z. Guo, Maraging Steels: Modelling of Microstructure, Properties, and Applications. Woodhead, Oxford, 2009.

[30] R. F. Decker, J. T. Eash, and A. J. Goldman, "18\% Nickel maraging steel," Trans. ASM, vol. 55, no. 1, p. 58, 1962.

[31] Y. He, K. Yang, and W. Sha, "Microstructure and mechanical properties of a 2000 MPa grade co-free maraging steel," Metall. Mater. Trans. A, vol. 36, no. 9, pp. 2273-2287, 2005.

[32] H. Leitner, M. Schober, and R. Schnitzer, "Splitting phenomenon in the precipitation evolution in an $\mathrm{Fe}-\mathrm{Ni}-\mathrm{Al}-\mathrm{Ti}-\mathrm{Cr}$ stainless steel," Acta Mater., vol. 58, no. 4, pp. 1261-1269, 2010.

[33] S. J. Kim and C. M. Wayman, "Strengthening behaviour and embrittlement phenomena in Fe-Ni-Mn-(Ti) maraging alloys," Mater. Sci. Eng. A, vol. 207, no. 1, pp. 22-29, 1996.

[34] S. Lee, S.-J. Lee, and B. C. De Cooman, "Austenite stability of ultrafinegrained transformation-induced plasticity steel with Mn partitioning," Scr. Mater., vol. 65, no. 3, pp. 225-228, Aug. 2011.

[35] Y. Tomita and T. Okawa, "Effect of microstructure on mechanical properties of isothermally bainite-transformed 300M steel," Mater. Sci. Eng. A, vol. 172, no. 1-2, pp. 145-151, Nov. 1993. 
[36] H. Springer, M. Belde, and D. Raabe, "Bulk combinatorial design of ductile martensitic stainless steels through confined martensite-to-austenite reversion," Mater. Sci. Eng. A, vol. 582, pp. 235-244, Oct. 2013.

[37] E. A. Wilson, "Discussion of 'precipitation of austenite particles at grain boundaries during aging of Fe-Mn-Ni steel,"' Metall. Mater. Trans. A, vol. 35, no. 1, pp. 352-355, Jan. 2004.

[38] H. Leitner, R. Schnitzer, M. Schober, and S. Zinner, "Precipitate modification in PH13-8 Mo type maraging steel," Acta Mater., vol. 59, no. 12, pp. 50125022, 2011.

[39] R. Schnitzer, M. Schober, S. Zinner, and H. Leitner, "Effect of Cu on the evolution of precipitation in an $\mathrm{Fe}-\mathrm{Cr}-\mathrm{Ni}-\mathrm{Al}-\mathrm{Ti}$ maraging steel," Acta Mater., vol. 58, no. 10, pp. 3733-3741, 2010.

[40] S. Höring, N. Wanderka, and J. Banhart, "The influence of $\mathrm{Cu}$ addition on precipitation in Fe-Cr-Ni-Al-(Cu) model alloys.," Ultramicroscopy, vol. 109, no. 5, pp. 574-9, Apr. 2009.

[41] F. G. Wilson and F. B. Pickering, "A study of precipitation following reversion and high temperature ageing in a $25 \% \mathrm{Ni}, 15 \% \mathrm{Cr}, 4 \% \mathrm{Ti}$ steel," Acta Metall., vol. 18, no. 7, pp. 775-783, 1970.

[42] M. Hättestrand, J.-O. Nilsson, K. Stiller, P. Liu, and M. Andersson, "Precipitation hardening in a $12 \% \mathrm{Cr}-9 \% \mathrm{Ni}-4 \% \mathrm{Mo}-2 \% \mathrm{Cu}$ stainless steel," Acta Mater., vol. 52, no. 4, pp. 1023-1037, 2004.

[43] P. Liu, A. H. Stigenberg, and J. O. Nilsson, "Quasicrystalline and crystalline precipitation during isothermal tempering in a $12 \mathrm{Cr}-9 \mathrm{Ni}-4 \mathrm{Mo}$ maraging stainless steel," Acta Metall. Mater., vol. 43, no. 7, pp. 2881-2890, 1995.

[44] D. Raabe, D. Ponge, O. Dmitrieva, and B. Sander, "Nanoprecipitate-hardened 1.5 GPa steels with unexpected high ductility," Scr. Mater., vol. 60, no. 12, pp. 1141-1144, 2009.

[45] Y. R. Wen, A. Hirata, Z. W. Zhang, T. Fujita, C. T. Liu, J. H. Jiang, and M. W. Chen, "Microstructure characterization of $\mathrm{Cu}$-rich nanoprecipitates in a $\mathrm{Fe}-$ 2.5 Cu-1.5 Mn-4.0 Ni-1.0 Al multicomponent ferritic alloy," Acta Mater., vol. 61, no. 6, pp. 2133-2147, 2013.

[46] E. V Pereloma, R. A. Stohr, M. K. Miller, and S. P. Ringer, “Observation of precipitation evolution in $\mathrm{Fe}-\mathrm{Ni}-\mathrm{Mn}-\mathrm{Ti}-\mathrm{Al}$ maraging steel by atom probe tomography," Metall. Mater. Trans. A Phys. Metall. Mater. Sci., vol. 40, no. 13, pp. 3069-3075, 2009. 
[47] L.-T. Shiang and C. M. Wayman, "Maragin behavior of an Fe-19.5Ni-5Mn alloy. III: Mechanical properties," Metallography, vol. 22, no. 1, pp. 15-36, Jan. 1989.

[48] E. V Pereloma, A. Shekhter, M. K. Miller, and S. P. Ringer, "Ageing behaviour of an $\mathrm{Fe}-20 \mathrm{Ni}-1.8 \mathrm{Mn}-1.6 \mathrm{Ti}-0.59 \mathrm{Al}$ (wt\%) maraging alloy: clustering, precipitation and hardening," Acta Mater., vol. 52, no. 19, pp. 5589-5602, 2004.

[49] N. H. Heo, J. W. Nam, Y. U. Heo, and S. J. Kim, "Grain boundary embrittlement by $\mathrm{Mn}$ and eutectoid reaction in binary Fe-12Mn steel," Acta Mater., vol. 61, no. 11, pp. 4022-4034, 2013.

[50] D. Raabe, S. Sandlöbes, J. Millán, D. Ponge, H. Assadi, M. Herbig, and P.-P. Choi, "Segregation engineering enables nanoscale martensite to austenite phase transformation at grain boundaries: A pathway to ductile martensite," Acta Mater., vol. 61, no. 16, pp. 6132-6152, Sep. 2013.

[51] W. Sha, A. Cerezo, and G. Smith, "Phase chemistry and precipitation reactions in maraging steels: Part I. Introduction and study of Co-containing C-300 steel," Metall. Mater. Trans. A, vol. 24, no. 6, pp. 1221-1232, 1993.

[52] S.-J. Kim and C. M. Wayman, "Precipitation behavior and microstructural changes in maraging Fe-Ni-Mn-Ti alloys," Mater. Sci. Eng. A, vol. 128, no. 2, pp. 217-230, 1990.

[53] A. Shekhter, H. Aaronson, M. Miller, S. Ringer, and E. Pereloma, "Effect of aging and deformation on the microstructure and properties of $\mathrm{Fe}-\mathrm{Ni}-\mathrm{Ti}$ maraging steel," Metall. Mater. Trans. A, vol. 35, no. 3, pp. 973-983, 2004.

[54] V. K. Vasudevan, S. J. Kim, and C. M. Wayman, "Precipitation reactions and strengthening behavior in $18 \mathrm{Wt}$ Pct nickel maraging steels," Metall. Trans. A, vol. 21, no. 10, pp. 2655-2668, Oct. 1990.

[55] R. J. Rioja and D. E. LAughlin, "The early stages of GP zone formation in naturally aged Ai-4 wt pet cu alloys," Metall. Trans. A, vol. 8, no. 8, pp. 1257-1261, Aug. 1977.

[56] W. Sha, A. Cerezo, and G. D. W. Smith, "Phase chemistry and precipitation reactions in maraging steels: Part IV. Discussion and conclusions," Metall. Trans. A, vol. 24, no. 6, pp. 1251-1256, 1993.

[57] C. Servant, G. Maeder, and G. Cizeron, "A small-angle x-ray scattering investigation of the zone formation of 'maraging' type alloys," Metall. Trans. A, vol. 6, no. 5, pp. 981-990, May 1975. 
[58] C. Servant, N. Bouzid, and O. Lyon, "Small-angle X-ray scattering investigation of the martensite unmixing in maraging alloy using synchrotron radiation," Philos. Mag. A, vol. 56, no. 5, pp. 565-582, Nov. 1987.

[59] A. Gemperle, J. Gemperlova, W. Sha, and G. D. W. Smith, "Aging behaviour of cobalt free chromium containing maraging steels," Mater. Sci. Technol., vol. 8, no. 6, pp. 546-554, 1992.

[60] Z. K. Teng, C. T. Liu, G. Ghosh, P. K. Liaw, and M. E. Fine, "Effects of Al on the microstructure and ductility of NiAl-strengthened ferritic steels at room temperature," Intermetallics, vol. 18, no. 8, pp. 1437-1443, Aug. 2010.

[61] Z. K. Teng, F. Zhang, M. K. Miller, C. T. Liu, S. Huang, Y. T. Chou, R. H. Tien, Y. A. Chang, and P. K. Liaw, "New NiAl-strengthened ferritic steels with balanced creep resistance and ductility designed by coupling thermodynamic calculations with focused experiments," Intermetallics, vol. 29, pp. 110-115, Oct. 2012.

[62] Z. Sun, C. H. Liebscher, S. Huang, Z. Teng, G. Song, G. Wang, M. Asta, M. Rawlings, M. E. Fine, and P. K. Liaw, "New design aspects of creep-resistant NiAl-strengthened ferritic alloys," Scr. Mater., vol. 68, no. 6, pp. 384-388, 2013.

[63] H. K. D. H. Bhadeshia, "Design of ferritic creep-resistant steels," ISIJ Int., vol. 41, no. 6, pp. 626-640, 2001.

[64] R. Viswanathan, J. F. Henry, J. Tanzosh, G. Stanko, J. Shingledecker, B. Vitalis, and R. Purgert, "U.S. Program on Materials Technology for UltraSupercritical Coal Power Plants," J. Mater. Eng. Perform., vol. 14, no. 3, pp. 281-292, Jun. 2005.

[65] G. Eggeler, "The effect of long-term creep on particle coarsening in tempered martensite ferritic steels," Acta Metall., vol. 37, no. 12, pp. 3225-3234, Dec. 1989.

[66] J. Pešička, R. Kužel, A. Dronhofer, and G. Eggeler, "The evolution of dislocation density during heat treatment and creep of tempered martensite ferritic steels," Acta Mater., vol. 51, no. 16, pp. 4847-4862, Sep. 2003.

[67] A. Kostka, K. Tak, R. Hellmig, Y. Estrin, and G. Eggeler, "On the contribution of carbides and micrograin boundaries to the creep strength of tempered martensite ferritic steels," Acta Mater., vol. 55, no. 2, pp. 539-550, Jan. 2007.

[68] Y. Wang, K.-H. Mayer, A. Scholz, C. Berger, H. Chilukuru, K. Durst, and W. Blum, "Development of new $11 \% \mathrm{Cr}$ heat resistant ferritic steels with enhanced creep resistance for steam power plants with operating steam 
temperatures up to $650^{\circ}$ C," Mater. Sci. Eng. A, vol. 510-511, pp. 180-184, Jun. 2009.

[69] A. Aghajani, C. Somsen, and G. Eggeler, "On the effect of long-term creep on the microstructure of a $12 \%$ chromium tempered martensite ferritic steel," Acta Mater., vol. 57, no. 17, pp. 5093-5106, Oct. 2009.

[70] K. SC, "Precipitation processes in Fe-base ferritic NiAl alloys," Northwestern University, 1971.

[71] H. A. Calderon, M. E. Fine, and J. R. Weertman, "Coarsening and morphology of $\beta^{\prime}$ particles in Fe-Ni-Al-Mo ferritic alloys," Metall. Trans. A, vol. 19, no. 5, pp. 1135-1146, May 1988.

[72] S. M. Zhu, S. C. Tjong, and J. K. L. Lai, "Creep behavior of a $\beta^{\prime}(\mathrm{NiAl})$ precipitation strengthened ferritic $\mathrm{Fe}-\mathrm{Cr}-\mathrm{Ni}-\mathrm{Al}$ alloy," Acta Mater., vol. 46, no. 9, pp. 2969-2976, May 1998.

[73] C. Stallybrass and G. Sauthoff, "Ferritic Fe-Al-Ni-Cr alloys with coherent precipitates for high-temperature applications," Mater. Sci. Eng. A, vol. 387389, pp. 985-990, Dec. 2004.

[74] C. Stallybrass, A. Schneider, and G. Sauthoff, "The strengthening effect of $(\mathrm{Ni}, \mathrm{Fe}) \mathrm{Al}$ precipitates on the mechanical properties at high temperatures of ferritic Fe-Al-Ni-Cr alloys," Intermetallics, vol. 13, no. 12, pp. 1263-1268, Dec. 2005.

[75] W. B. Pearson, Handbook of Lattice Spacing and Structure of Metals. Pergamon Press, 1958.

[76] R. Taillard, A. Pineau, and B. J. Thomas, "The precipitation of the intermetallic compound NiAl in Fe-19wt.\%Cr alloys," Mater. Sci. Eng., vol. 54, no. 2, pp. 209-219, Jul. 1982.

[77] Z. K. Teng, G. Ghosh, M. K. Miller, S. Huang, B. Clausen, D. W. Brown, and P. K. Liaw, "Neutron-diffraction study and modeling of the lattice parameters of a NiAl-precipitate-strengthened Fe-based alloy," Acta Mater., vol. 60, no. 13-14, pp. 5362-5369, Aug. 2012.

[78] M. Schober, R. Schnitzer, and H. Leitner, "Precipitation evolution in a Ti-free and Ti-containing stainless maraging steel," Ultramicroscopy, vol. 109, no. 5, pp. 553-562, 2009.

[79] H. C. Burnett, R. H. Duff, and H. C. Vacher, "Identification of Metallurgical Reactions and Their Effect on the Mechanical Properties of 17-7 PH Stainless Steel," J. Res. Natl. Bur. Stand., vol. 66C, no. 2, 1962. 
[80] V. Seetharaman, M. Sundararaman, and R. Krishnan, "Precipitation hardening in a PH 13-8 Mo stainless steel," Mater. Sci. Eng., vol. 47, no. 1, pp. 1-11, 1981.

[81] Z. Guo, W. Sha, and D. Vaumousse, "Microstructural evolution in a PH13-8 stainless steel after ageing," Acta Mater., vol. 51, no. 1, pp. 101-116, 2003.

[82] D. H. Ping, M. Ohnuma, Y. Hirakawa, Y. Kadoya, and K. Hono, "Microstructural evolution in $13 \mathrm{Cr}-8 \mathrm{Ni}-2.5 \mathrm{Mo}-2 \mathrm{Al}$ martensitic precipitationhardened stainless steel," Mater. Sci. Eng. A, vol. 394, no. 1-2, pp. 285-295, 2005 .

[83] A. Gemperle, J. Gemperlová, W. Sha, and G. D. W. Smith, “Aging behaviour of cobalt free chromium containing maraging steels," Mater. Sci. Technol., vol. 8, no. 6, pp. 546-554, Jun. 1992.

[84] H. Leitner, M. Schober, R. Schnitzer, and S. Zinner, "Strengthening behavior of $\mathrm{Fe}-\mathrm{Cr}-\mathrm{Ni}-\mathrm{Al}-(\mathrm{Ti})$ maraging steels," Mater. Sci. Eng. A, vol. 528, no. 15, pp. 5264-5270, Jun. 2011.

[85] C. H. Liebscher, V. Radmilovic, U. Dahmen, M. Asta, and G. Ghosh, "On the formation of hierarchically structured L2 1 -Ni2TiAl type precipitates in a ferritic alloy," J. Mater. Sci., vol. 48, no. 5, pp. 2067-2075, 2013.

[86] L. A. Bendersky, P. W. Voorhees, W. J. Boettinger, and W. C. Johnson, "The role of elastic energy in the morphological development of a NiTiAl alloy," Scr. Metall., vol. 22, no. 7, pp. 1029-1034, Jan. 1988.

[87] W. J. Boettinger, L. A. Bendersky, F. S. Biancaniello, and J. W. Cahn, "Rapid solidification and ordering of B2 and L21 phases in the NiAl-NiTi system," Mater. Sci. Eng., vol. 98, pp. 273-276, Feb. 1988.

[88] R. D. Field, R. Darolia, and D. F. Lahrman, "Precipitation in NiAl/Ni2AlTi alloys," Scr. Metall., vol. 23, no. 9, pp. 1469-1474, Sep. 1989.

[89] J. Jung, G. Ghosh, D. Isheim, and G. B. Olson, "Precipitation of heusler phase (Ni2TiAl) from B2-TiNi in Ni-Ti-Al and Ni-Ti-Al-X (X=Hf, Zr) alloys," Metall. Mater. Trans. A, vol. 34, no. 6, pp. 1221-1235, 2003.

[90] J. Jung, G. Ghosh, and G. B. Olson, “A comparative study of precipitation behavior of Heusler phase (Ni2TiAl) from B2-TiNi in Ni-Ti-Al and Ni-Ti$\mathrm{Al}-\mathrm{X}$ (X=Hf, Pd, Pt, Zr) alloys," Acta Mater., vol. 51, no. 20, pp. 6341-6357, 2003.

[91] P. R. Strutt, R. S. Polvani, and J. C. Ingram, "Creep behavior of the heusler type structure alloy Ni2AlTi," Metall. Trans. A, vol. 7, no. 1, pp. 23-31, Dec. 1976. 
[92] N.-H. Heo, "Theory of nonequilibrium segregation in an Fe-Mn-Ni ternary alloy and a ductile-brittle-ductile transition," Acta Mater., vol. 44, no. 7, pp. 3015-3023, Jul. 1996.

[93] Y. M. Kim, C. D. Yim, and B. S. You, "Grain refining mechanism in Mg-Al base alloys with carbon addition," Scr. Mater., vol. 57, no. 8, pp. 691-694, Oct. 2007.

[94] R.-Y. Li, J.-Y. Qin, T.-K. Gu, and X.-F. Bian, "Structure of liquid Al80Mn20 alloy by Reverse Monte Carlo simulation," J. Non. Cryst. Solids, vol. 354, no. 15-16, pp. 1736-1739, Mar. 2008.

[95] D. R. Squires and E. A. Wilson, "Aging and brittleness in an Fe-Ni-Mn alloy," Metall. Trans., vol. 3, no. 2, pp. 579-585, Feb. 1972.

[96] D. R. Squires, F. G. Wilson, and E. A. Wilson, "The influence of Mo and Co on the embrittlement of an Fe-Ni-Mn alloy," Metall. Trans., vol. 5, no. 12, pp. 2569-2578, Dec. 1974.

[97] Z. W. Zhang, C. T. Liu, X.-L. Wang, K. C. Littrell, M. K. Miller, K. An, and B. A. Chin, "From embryos to precipitates: A study of nucleation and growth in a multicomponent ferritic steel," Phys. Rev. B, vol. 84, no. 17, p. 174114, Nov. 2011.

[98] G. Fourlaris, A. J. Baker, and G. D. Papadimitriou, "Microscopic characterisation of $\varepsilon$-Cu interphase precipitation in hypereutectoid $\mathrm{Fe}-\mathrm{C}-\mathrm{Cu}$ alloys," Acta Metall. Mater., vol. 43, no. 7, pp. 2589-2604, Jul. 1995.

[99] M. D. Mulholland and D. N. Seidman, "Nanoscale co-precipitation and mechanical properties of a high-strength low-carbon steel," Acta Mater., vol. 59, no. 5, pp. 1881-1897, Mar. 2011.

[100] N. Maruyama, M. Sugiyama, T. Hara, and H. Tamehiro, "Precipitation and Phase Transformation of Copper Particles in Low Alloy Ferritic and Martensitic Steels," Mater. Trans. JIM, vol. 40, 1999.

[101] A. Ghosh, S. das, and S. Chatterjee, "Ageing behavior of a Cu-bearing ultrahigh strength steel," Mater. Sci. Eng. A, vol. 486, no. 1-2, pp. 152-157, Jul. 2008 .

[102] R. Prakash Kolli and D. N. Seidman, "The temporal evolution of the decomposition of a concentrated multicomponent Fe-Cu-based steel," Acta Mater., vol. 56, no. 9, pp. 2073-2088, May 2008.

[103] Z. Zhang, C. T. Liu, M. K. Miller, X.-L. Wang, Y. Wen, T. Fujita, A. Hirata, M. Chen, G. Chen, and B. A. Chin, "A nanoscale co-precipitation approach for property enhancement of Fe-base alloys.," Sci. Rep., vol. 3, p. 1327, Jan. 2013. 
[104] M. Kapoor, "Design and Development of bcc - Cu - and B2 NiAl Precipitation - Strengthened Ferritic Steel," Northwestern University, 2013.

[105] P. J. Othen, M. L. Jenkins, and G. D. W. Smith, "High-resolution electron microscopy studies of the structure of Cu precipitates in $\alpha$-Fe," Philos. Mag. A, vol. 70, no. 1, pp. 1-24, Jul. 1994.

[106] Z. B. Jiao, J. H. Luan, Z. W. Zhang, M. K. Miller, W. B. Ma, and C. T. Liu, "Synergistic effects of $\mathrm{Cu}$ and $\mathrm{Ni}$ on nanoscale precipitation and mechanical properties of high-strength steels," Acta Mater., vol. 61, no. 16, pp. 59966005, Sep. 2013.

[107] K. Stiller, M. Hättestrand, and F. Danoix, "Precipitation in $9 \mathrm{Ni}-12 \mathrm{Cr}-2 \mathrm{Cu}$ maraging steels," Acta Mater., vol. 46, no. 17, pp. 6063-6073, 1998.

[108] N. Maruyama, M. Sugiyama, T. Hara, and H. Tamehiro, "Precipitation and phase transformation of copper particles in low alloy ferritic and martensitic steels," Mater. Trans. JIM, vol. 40, no. 4, pp. 268-277, 1999.

[109] Y. R. Wen, Y. P. Li, A. Hirata, Y. Zhang, T. Fujita, T. Furuhara, C. T. Liu, A. Chiba, and M. W. Chen, "Synergistic alloying effect on microstructural evolution and mechanical properties of $\mathrm{Cu}$ precipitation-strengthened ferritic alloys," Acta Mater., vol. 61, no. 20, pp. 7726-7740, Dec. 2013.

[110] M. K. Miller, B. D. Wirth, and G. R. Odette, "Precipitation in neutronirradiated $\mathrm{Fe}-\mathrm{Cu}$ and $\mathrm{Fe}-\mathrm{Cu}-\mathrm{Mn}$ model alloys: a comparison of APT and SANS data," Mater. Sci. Eng. A, vol. 353, no. 1-2, pp. 133-139, Jul. 2003.

[111] L. T. Shiang and C. M. Wayman, "Maraging behavior in an Fe-19.5Ni-5Mn alloy I: Precipitation characteristics," Metallography, vol. 21, no. 4, pp. 399423, Nov. 1988.

[112] S.-J. Kim and C. M. Wayman, "Electron microscopy study of precipitates in Fe-Ni-Mn maraging alloys," Mater. Sci. Eng. A, vol. 136, pp. 121-129, Apr. 1991.

[113] S.-H. Mun, M. Watanabe, D. B. Williams, X. Li, K. Hwan Oh, and H.-C. Lee, "Precipitation of austenite particles at grain boundaries during aging of $\mathrm{Fe}$ Mn-Ni steel," Metall. Mater. Trans. A, vol. 33, no. 4, pp. 1057-1067, Apr. 2002.

[114] J. Singh and C. M. Wayman, "Age-hardening characteristics of a martensitic Fe-Ni-Mn alloy," Mater. Sci. Eng., vol. 94, pp. 233-242, Oct. 1987.

[115] H. C. Feng, E. A. Wilson, and C. J. McMahon, in 3rd Int. Conf. on the Strength of Metals and Alloys, 1973, p. 129. 
[116] H. Suto and T. Murakami, "On the Grain Boundary Embrittlement in an Aged 12Ni-6Mn-Fe Martensite,” Trans. Japan Inst. Met., vol. 20, p. 365, 1979.

[117] N.-H. Heo and H.-C. Lee, "The embrittlement and de-embrittlement of grain boundaries in an Fe-Mn-Ni alloy due to grain boundary segregation of Mn," Metall. Mater. Trans. A, vol. 27, no. 4, pp. 1015-1020, Apr. 1996.

[118] H.-C. Lee, S.-H. Mun, and D. McKenzie, "Electron microscopy study on the grain-boundary precipitation during aging of Fe-10Ni-5Mn steel," Metall. Mater. Trans. A, vol. 34, no. 11, pp. 2421-2428, Nov. 2003.

[119] S. Hossein Nedjad, M. Nili Ahmadabadi, and T. Furuhara, "Transmission Electron Microscopy Study on the Grain Boundary Precipitation of an Fe-NiMn Maraging Steel," Metall. Mater. Trans. A, vol. 39, no. 1, pp. 19-27, 2008.

[120] S. Hossein Nedjad, M. Nili Ahmadabadi, and T. Furuhara, "Correlation between the intergranular brittleness and precipitation reactions during isothermal aging of an Fe-Ni-Mn maraging steel," Mater. Sci. Eng. A, vol. 490, no. 1-2, pp. 105-112, 2008.

[121] F. Nikbakht, M. Nasim, C. Davies, E. A. Wilson, and H. Adrian, "Isothermal embrittlement of Fe-8Mn alloys at $450^{\circ} \mathrm{C}$," Mater. Sci. Technol., vol. 26, no. 5, pp. 552-558, May 2010.

[122] M. Nasim, B. C. Edwards, and E. A. Wilson, "A study of grain boundary embrittlement in an Fe-8\%Mn alloy," Mater. Sci. Eng. A, vol. 281, no. 1-2, pp. 56-67, Apr. 2000.

[123] J. K. Tien, P. G. Shewmon, and J. S. Foster, "On evaluating the duration of the interfacial rate controlling step in reactions involving diffusion," Scr. Metall., vol. 7, no. 11, pp. 1171-1174, Nov. 1973.

[124] R. Wagner, R. Kampmann, and P. W. Voorhees, "Homogeneous SecondPhase Precipitation, in Phase Transformations in Materials," G. Kostorz, Ed. Weinheim, FRG: Wiley-VCH Verlag GmbH \& Co. KGaA, 2001.

[125] R. Kampmann and R. Wagner, Decomposition of Alloys: the Early Stages. Elsevier, 1984.

[126] I. M. Lifshitz and V. V. Slyozov, "The kinetics of precipitation from supersaturated solid solutions," J. Phys. Chem. Solids, vol. 19, no. 1-2, pp. 35-50, Apr. 1961.

[127] C. Wagner, "Theorie der Alterung von Niederschlägen durch Umlösen (Ostwald-Reifung)," Zeitschrift für Elektrochemie, Berichte der Bunsengesellschaft für Phys. Chemie, vol. 65, no. 7-8, pp. 581-591, Sep. 1961. 
[128] L. Ratke and P. W. Voorhees, Growth and Coarsening: Ripening in Materials Processing, Springer V. Berlin, 2002.

[129] A. J. Ardell, "An application of the theory of particle coarsening: The $\gamma$ ' precipitate in Ni-Al alloys," Acta Metall., vol. 16, no. 4, pp. 511-516, Apr. 1968.

[130] C. FULLER and D. SEIDMAN, "Temporal evolution of the nanostructure of $\mathrm{Al}(\mathrm{Sc}, \mathrm{Zr})$ alloys: Part II-coarsening of $\mathrm{Al}(\mathrm{ScZr})$ precipitates," Acta Mater., vol. 53, no. 20, pp. 5415-5428, Dec. 2005.

[131] N. Bouzid, C. Servant, and O. Lyon, "Anomalous small-angle X-ray scattering from a maraging alloy during martensite unmixing," Philos. Mag. Part B, vol. 57, no. 3, pp. 343-359, Mar. 1988.

[132] E.-W. Huang, P. K. Liaw, L. Porcar, Y. Liu, Y.-L. Liu, J.-J. Kai, and W.-R. Chen, "Study of nanoprecipitates in a nickel-based superalloy using smallangle neutron scattering and transmission electron microscopy," Appl. Phys. Lett., vol. 93, no. 16, p. 161904, Oct. 2008.

[133] a. Deschamps, L. Lae, and P. Guyot, "In situ small-angle scattering study of the precipitation kinetics in an $\mathrm{Al}-\mathrm{Zr}-\mathrm{Sc}$ alloy," Acta Mater., vol. 55, no. 8, pp. 2775-2783, May 2007.

[134] P. Donnadieu, Y. Shao, F. De Geuser, G. A. Botton, S. Lazar, M. Cheynet, M. de Boissieu, and A. Deschamps, "Atomic structure of T1 precipitates in Al$\mathrm{Li}-\mathrm{Cu}$ alloys revisited with HAADF-STEM imaging and small-angle X-ray scattering," Acta Mater., vol. 59, no. 2, pp. 462-472, 2011.

[135] J. Millán, D. Ponge, D. Raabe, P. Choi, and O. Dmitrieva, "Characterization of Nano-Sized Precipitates in a Mn-Based Lean Maraging Steel by Atom Probe Tomography," Steel Res. Int., vol. 82, no. 2, pp. 137-145, Feb. 2011.

[136] A. . Brailsford and P. Wynblatt, "The dependence of ostwald ripening kinetics on particle volume fraction," Acta Metall., vol. 27, no. 3, pp. 489-497, Mar. 1979.

[137] J. A. Marqusee and J. Ross, "Theory of Ostwald ripening: Competitive growth and its dependence on volume fraction," J. Chem. Phys., vol. 80, no. 1, p. 536, Jan. 1984.

[138] K. Tsumuraya and Y. Miyata, "Coarsening models incorporating both diffusion geometry and volume fraction of particles," Acta Metall., vol. 31, no. 3, pp. 437-452, Mar. 1983.

[139] S. P. Marsh and M. E. Glicksman, "Kinetics of phase coarsening in dense systems," Acta Mater., vol. 44, no. 9, pp. 3761-3771, Sep. 1996. 
[140] H. Ghassemi-Armaki, R. Maaß, S. P. Bhat, S. Sriram, J. R. Greer, and K. S. Kumar, "Deformation response of ferrite and martensite in a dual-phase steel," Acta Mater., 2013.

[141] J.-B. Seol, D. Raabe, P.-P. Choi, Y.-R. Im, and C.-G. Park, "Atomic scale effects of alloying, partitioning, solute drag and austempering on the mechanical properties of high-carbon bainitic-austenitic TRIP steels," Acta Mater., vol. 60, no. 17, pp. 6183-6199, Oct. 2012.

[142] S. Zhang and K. O. Findley, "Quantitative assessment of the effects of microstructure on the stability of retained austenite in TRIP steels," Acta Mater., vol. 61, no. 6, pp. 1895-1903, Apr. 2013.

[143] S. Zaefferer, J. Ohlert, and W. Bleck, "A study of microstructure, transformation mechanisms and correlation between microstructure and mechanical properties of a low alloyed TRIP steel," Acta Mater., vol. 52, no. 9, pp. 2765-2778, May 2004.

[144] W. Bleck, "Proceedings of the International Conference on TRIP-Aided High Strength Ferrous Alloys," 2002, p. 13.

[145] A. Markfeld and A. Rosen, "The effect of reverted austenite on the plastic deformation of maraging steel," Mater. Sci. Eng., vol. 46, no. 2, pp. 151-157, Dec. 1980.

[146] L. T. Shiang and C. M. Wayman, "Maraging behavior of an Fe-19.5Ni-5Mn alloy II: Evolution of reverse-transformed austenite during overaging," Metallography, vol. 21, no. 4, pp. 425-451, 1988.

[147] R. Schnitzer, R. Radis, M. Nöhrer, M. Schober, R. Hochfellner, S. Zinner, E. Povoden-Karadeniz, E. Kozeschnik, and H. Leitner, "Reverted austenite in PH 13-8 Mo maraging steels," Mater. Chem. Phys., vol. 122, no. 1, pp. 138-145, 2010 .

[148] S. Hossein Nedjad, M. R. Movaghar Garabagh, M. Nili Ahmadabadi, and H. Shirazi, "Effect of further alloying on the microstructure and mechanical properties of an Fe-10Ni-5Mn maraging steel," Mater. Sci. Eng. A, vol. 473, no. 1-2, pp. 249-253, 2008.

[149] A. Shekhter, H. I. Aaronson, M. K. Miller, S. P. Ringer, and E. V. Pereloma, "Effect of aging and deformation on the microstructure and properties of Fe-Ni-Ti maraging steel," Metall. Mater. Trans. A, vol. 35, no. 13, pp. 973983, Mar. 2004.

[150] Y. He, K. Yang, W. Sha, and D. J. Cleland, "Microstructure and mechanical properties of a $2000 \mathrm{MPa}$ Co-free maraging steel after aging at $753 \mathrm{~K}$," Metall. Mater. Trans. A, vol. 35, no. 9, pp. 2747-2755, 2004. 
[151] Y. He, K. Yang, K. Liu, W. Sha, and Z. Guo, "Age hardening and mechanical properties of a $2400 \mathrm{MPa}$ grade cobalt-free maraging steel," Metall. Mater. Trans. A, vol. 37, no. 4, pp. 1107-1116, 2006.

[152] R. Schnitzer, G. A. Zickler, E. Lach, H. Clemens, S. Zinner, T. Lippmann, and H. Leitner, "Influence of reverted austenite on static and dynamic mechanical properties of a PH 13-8 Mo maraging steel," Mater. Sci. Eng. A, vol. 527, no. 7-8, pp. 2065-2070, 2010.

[153] V. P. Vylezhnev, A. A. Sukhikh, V. G. Bragin, and S. A. Kokovyakina, "Mechanical properties of maraging N18K9M5T steel with residual and reverted austenite," Phys. Met. Metallogr., vol. 75, p. 448, 1993.

[154] D. Duchateau and M. Guttmann, “An X-ray microanalysis study of the partition of manganese in 6 and 9\% Mn cryogenic steels and its influence on the stability of austenite dispersed in a tempered martensitic matrix," Acta Metall., vol. 29, no. 7, pp. 1291-1297, Jul. 1981.

[155] G. N. Haidemenopoulos and A. N. Vasilakos, "On the thermodynamic stability of retained austenite in 4340 steel," J. Alloys Compd., vol. 247, no. 1-2, pp. 128-133, Jan. 1997.

[156] C. K. Syn, B. Fultz, and J. W. Morris, "Mechanical stability of retained austenite in tempered 9Ni steel," Metall. Trans. A, vol. 9, no. 11, pp. 16351640, Nov. 1978.

[157] P. J. Jacques, F. Delannay, and J. Ladrière, "On the influence of interactions between phases on the mechanical stability of retained austenite in transformation-induced plasticity multiphase steels," Metall. Mater. Trans. A, vol. 32, no. 11, pp. 2759-2768, Nov. 2001.

[158] J. Wang and S. Van Der Zwaag, "Stabilization mechanisms of retained austenite in transformation-induced plasticity steel," Metall. Mater. Trans. A, vol. 32, no. 6, pp. 1527-1539, Jun. 2001.

[159] D. Q. Bai, A. Di Chiro, and S. Yue, "Stability of Retained Austenite in a Nb Microalloyed Mn-Si TRIP Steel," in Materials Science Forum, 1998, vol. 284-286, pp. 253-262.

[160] J. A. Jiménez, M. Carsí, O. A. Ruano, and G. Frommeyer, "Effect of testing temperature and strain rate on the transformation behaviour of retained austenite in low-alloyed multiphase steel," Mater. Sci. Eng. A, vol. 508, no. 12, pp. 195-199, May 2009.

[161] L. Samek, E. Moor, J. Penning, and B. C. Cooman, "Influence of alloying elements on the kinetics of strain-induced martensitic nucleation in low-alloy, multiphase high-strength steels," Metall. Mater. Trans. A, vol. 37, no. 1, pp. 109-124, Jan. 2006. 
[162] H. YANG and H. BHADESHIA, "Austenite grain size and the martensitestart temperature," Scr. Mater., vol. 60, no. 7, pp. 493-495, Apr. 2009.

[163] E. Jimenez-Melero, N. H. van Dijk, L. Zhao, J. Sietsma, S. E. Offerman, J. P. Wright, and S. van der Zwaag, "Martensitic transformation of individual grains in low-alloyed TRIP steels," Scr. Mater., vol. 56, no. 5, pp. 421-424, Mar. 2007.

[164] X. C. Xiong, B. Chen, M. X. Huang, J. F. Wang, and L. Wang, "The effect of morphology on the stability of retained austenite in a quenched and partitioned steel," Scr. Mater., vol. 68, no. 5, pp. 321-324, Mar. 2013.

[165] I. B. Timokhina, P. D. Hodgson, and E. V. Pereloma, "Effect of microstructure on the stability of retained austenite in transformation-inducedplasticity steels," Metall. Mater. Trans. A, vol. 35, no. 8, pp. 2331-2341, Aug. 2004.

[166] P. Wang, N. Xiao, S. Lu, D. Li, and Y. Li, "Investigation of the mechanical stability of reversed austenite in $13 \% \mathrm{Cr}-4 \% \mathrm{Ni}$ martensitic stainless steel during the uniaxial tensile test," Mater. Sci. Eng. A, vol. 586, pp. 292-300, Dec. 2013.

[167] J. I. Suk, S. H. Hong, and S. W. Nam, "Crystallographic orientation relationships among $\eta$-Ni3Ti precipitate, reverted austenite, and martensitic matrix in Fe-10Cr-10Ni-2W maraging alloy," Metall. Trans. A, vol. 24, no. 12, pp. 2643-2652, Dec. 1993.

[168] G. Brückner, J. Pospiech, I. Seidl, and G. Gottstein, "Orientation correlation during diffusional $\alpha \rightarrow \gamma$ phase transformation in a ferritic low carbon steel," Scr. Mater., vol. 44, no. 11, pp. 2635-2640, Jun. 2001.

[169] K. Verbeken, L. Barbé, and D. Raabe, "Evaluation of the Crystallographic Orientation Relationships between FCC and BCC Phases in TRIP Steels," ISIJ Int., vol. 49, no. 10, pp. 1601-1609, 2009.

[170] G. N. Haidemenopoulos and A. N. Vasilakos, "Modelling of austenite stability in low-alloy triple-phase steels," Steel Res., vol. 67, no. 11, pp. 513519, 1995.

[171] M.-M. Wang, C. C. Tasan, D. Ponge, A. Kostka, and D. Raabe, "Smaller is less stable: Size effects on twinning vs. transformation of reverted austenite in TRIP-maraging steels," Acta Mater., vol. 79, pp. 268-281, Oct. 2014.

[172] X. Li and Z. Yin, "Reverted austenite during aging in $18 \mathrm{Ni}(350)$ maraging steel," Mater. Lett., vol. 24, no. 4, pp. 239-242, 1995. 
[173] A. Grajcar, "Morphological features of retained austenite in thermomechanically processed C-Mn-Si-Al-Nb-Ti multiphase steel," J. Achiev. Mater. Manuf. Eng., vol. 39, no. 1, pp. 7-18, 2010.

[174] G. Kurdjumov and G. Sachs, "Over the mechanisms of steel hardening," $Z$. Phys, vol. 64, p. 325, 1930.

[175] Y. Mishima, R. M. Horn, V. F. Zackay, and E. R. Parker, "Direct decomposition of austenite in two Fe-V-C alloys," Metall. Trans. A, vol. 11, no. 3, pp. 431-440, Mar. 1980.

[176] Z. Nishiyama, "X-ray investigation of the mechanism of the transformation from face centered cubic lattice to body centered cubic," Sci. Rep. Tohoku Univ, vol. 23, p. 638, 1934.

[177] G Wassermann, "Influence of the--transformation of an irreversible Ni steel onto crystal orientation and tensile strength," Arch. Eisenhüttenwes, vol. 16, p. 647, 1933.

[178] W. Pitsch, "Der Orientierungszusammenhang zwischen Zementit und Austenit," Acta Metall., vol. 10, no. 9, pp. 897-900, 1962.

[179] L. Sandoval, H. M Urbassek, and P. Entel, "The Bain versus NishiyamaWassermann path in the martensitic transformation of Fe," New J. Phys., vol. 11, no. 10, p. 103027, Oct. 2009.

[180] E. C. Bain, Trans. AIME, vol. 70, p. 25, 1924.

[181] J. J. Jonas, Y. He, and S. Godet, "The possible role of partial dislocations in facilitating transformations of the Nishiyama-Wassermann type," Scr. Mater., vol. 52, no. 3, pp. 175-179, Feb. 2005.

[182] B. Verlinden, P. Bocher, E. Girault, and E. Aernoudt, "Austenite texture and bainite/austenite orientation relationships in TRIP steel," Scr. Mater., vol. 45, no. 8, pp. 909-916, Oct. 2001.

[183] D. Raabe, "Texture and microstructure evolution during cold rolling of a strip cast and of a hot rolled austenitic stainless steel," Acta Mater., vol. 45, no. 3, pp. 1137-1151, Mar. 1997.

[184] Y. Katz, H. Mathias, and S. Nadiv, "Mechanical stability of austenite in maraging steels," Metall. Trans. A, Phys. Metall. Mater. Sci., vol. 14 A, no. 5, pp. 801-808, 1983.

[185] J. I. Suk, S. H. Hong, and S. W. Nam, "The formation of twinned austenite in Fe10Cr10Ni2W maraging steel," Scr. Metall. Mater., vol. 25, no. 12, pp. 2651-2656, Dec. 1991. 
[186] L. T. Shiang and C. M. Wayman, "The formation of twinned Widmanstätten austenite," Metallography, vol. 18, no. 2, pp. 193-198, May 1985.

[187] K. Ameyama, T. Maki, and I. Tamura, "Morphology and crystallographic feature of intragranular gamma phase in (alpha+ gamma) two phase stainless steel," Nippon Kinzoku Gakkai-si, vol. 50, no. 1, pp. 10-19, 1986.

[188] B. B. He, M. X. Huang, Z. Y. Liang, A. H. W. Ngan, H. W. Luo, J. Shi, W. Q. Cao, and H. Dong, "Nanoindentation investigation on the mechanical stability of individual austenite grains in a medium-Mn transformation-induced plasticity steel," Scr. Mater., vol. 69, no. 3, pp. 215-218, Aug. 2013.

[189] J. J. Vlassak and W. D. Nix, "Measuring the elastic properties of anisotropic materials by means of indentation experiments," J. Mech. Phys. Solids, vol. 42, no. 8, pp. 1223-1245, Aug. 1994.

[190] M. Karlsen, Ø. Grong, M. Søfferud, J. Hjelen, G. Rørvik, and R. Chiron, "Scanning Electron Microscopy/Electron Backscatter Diffraction-Based Observations of Martensite Variant Selection and Slip Plane Activity in Supermartensitic Stainless Steels during Plastic Deformation at Elevated, Ambient, and Subzero Temperatures," Metall. Mater. Trans. A, vol. 40, no. 2, pp. 310-320, Jan. 2009.

[191] Y. You, C. Shang, L. Chen, and S. Subramanian, "Investigation on the crystallography of reverted structure and its effect on the properties of low carbon steel," Mater. Sci. Eng. A, vol. 546, pp. 111-118, Jun. 2012.

[192] T. Hara, N. Maruyama, Y. Shinohara, H. Asahi, G. Shigesato, M. Sugiyama, and T. Koseki, "Abnormal $\alpha$ to $\gamma$ Transformation Behavior of Steels with a Martensite and Bainite Microstructure at a Slow Reheating Rate," ISIJ Int., vol. 49, no. 11, pp. 1792-1800, 2009.

[193] M. R. Plichata and H. I. Aaronson, "Influence of Alloying Elements upon the Morphology of Austenite Formed From Martensite in Fe-C-X Alloys," Metall. Trans., vol. 5, no. 12, p. 2611, 1974.

[194] P. P. Sinha, D. Sivakumar, N. S. Babu, K. T. Tharian, and A. Natarajan, "Austenite reversion in $18 \mathrm{Ni}$ Co-free maraging steel," Steel Res., vol. 66, no. 11, pp. 490-494, 1995.

[195] K. Hirano, M. Cohen, and B. . Averbach, "Diffusion of nickel into iron," Acta Metall., vol. 9, no. 5, pp. 440-445, May 1961.

[196] J. Speer, D. K. Matlock, B. C. De Cooman, and J. G. Schroth, "Carbon partitioning into austenite after martensite transformation," Acta Mater., vol. 51, no. 9, pp. 2611-2622, May 2003. 
[197] R. W. K. Honeycombe and H. K. D. H. Bhadeshia, Steels: Microstructure and Properties. Oxford: Butterworth-Heinemann, 2006.

[198] N. Hansen, "Hall-Petch relation and boundary strengthening," Scr. Mater., vol. 51, no. 8, pp. 801-806, Oct. 2004.

[199] A. H. Cottrell, "Theory of brittle fracture in steel and similar metals," Trans. Metall. Soc. AIME, vol. 212, 1958.

[200] N. J. Petch, "The ductile-brittle transition in the fracture of $\alpha$-iron: I," Philos. Mag., vol. 3, no. 34, pp. 1089-1097, Oct. 1958.

[201] A.A. Griffith, "The theory of rupture," in Proc. 1st Int. Congr. Appl. Mech, 1924.

[202] N. S. Stoloff, in Fracture, H. Liebowitz, Ed. New York: Academic Press, 1969.

[203] Z. Guo, "The Limit of Strength and Toughness of Steel," University of California, Berkeley, 2001.

[204] J. W. Morris, Z. Guo, C. R. Krenn, and Y.-H. Kim, "The limits of strength and toughness in steel," ISIJ Int., vol. 41, no. 6, pp. 599-611.

[205] Z. Guo, K. Sato, T. K. Lee, and J. W. Morris, "Ultrafine grain size through thermal treatment of lath martensitic steels," in Ultrafine grained materials, 2000 .

[206] B. Fultz, J. I. Kim, Y. H. Kim, and J. W. Morris, "The chemical composition of precipitated austenite in 9Ni steel," Metall. Trans. A, vol. 17, no. 6, pp. 967-972, Jun. 1986.

[207] D. B. Williams and C. B. Carter, Transmission Electron Microscopy. New York: Springer Science, 2009.

[208] C. Yang, S. Luo, and W. Yu, GB: Retained austenite in steel-Quantitative determination-method of $X$-ray diffractometer. Standardization Administration of the People's Republic of China, 2006.

[209] W. Conshohocken, ASTM Standard, Standard Practice for X-Ray Determination of Retained Austenite in Steel with Near Random Crystallographic Orientation" Standard E975-03, vol. 03, no. Reapproved 2008. American Society for Testing and Materials, 2003.

[210] G. E. Dieter, Mechanical Metallurgy. NEW: New York: McGraw-Hill, 1986.

[211] J. R. Cahoon, "An improved equation relating hardness to ultimate strength," Metall. Trans., vol. 3, no. 11, p. 3040, 1972. 
[212] A. C. Fischer-Cripps, Nanoindentation. Springer New York, 2011.

[213] Y. Matsuoka, T. Iwasaki, N. Nakada, T. Tsuchiyama, and S. Takaki, "Effect of Grain Size on Thermal and Mechanical Stability of Austenite in Metastable Austenitic Stainless Steel," ISIJ Int., vol. 53, no. 7, pp. 1224-1230, Aug. 2013.

[214] H. Schumann, Neue Hütte, p. 12, 1970.

[215] J. Martínez, S. M. Cotes, A. F. Cabrera, J. Desimoni, and A. Fernández Guillermet, "On the relative fraction of $\varepsilon$ martensite in $\gamma$-Fe-Mn alloys," Mater. Sci. Eng. A, vol. 408, no. 1-2, pp. 26-32, 2005.

[216] P. Marinelli, A. Baruj, A. Fernández Guillermet, and M. Sade, "Lattice parameters of metastable structures in quenched Fe-Mn alloys. Part II: hcp phase," Zeitschrift fuer Met. Res. Adv. Tech., vol. 92, no. 5, pp. 489-493, 2001.

[217] Y.-U. Heo, M. Takeguchi, K. Furuya, and H.-C. Lee, "Discontinuous coarsening behavior of $\mathrm{Ni} 2 \mathrm{MnAl}$ intermetallic compound during isothermal aging treatment of $\mathrm{Fe}-\mathrm{Mn}-\mathrm{Ni}-\mathrm{Al}$ alloys," J. Electron Microsc. (Tokyo)., vol. 59, no. S1, pp. S135-S140, 2010.

[218] F. Moszner, A. S. Sologubenko, M. Schinhammer, C. Lerchbacher, A. C. Hänzi, H. Leitner, P. J. Uggowitzer, and J. F. Löffler, "Precipitation hardening of biodegradable Fe-Mn-Pd alloys," Acta Mater., vol. 59, no. 3, pp. 981-991, 2011.

[219] J. Millán, S. Sandlöbes, A. Al-Zubi, T. Hickel, P. Choi, J. Neugebauer, D. Ponge, and D. Raabe, "Designing Heusler nanoprecipitates by elastic misfit stabilization in Fe-Mn maraging steels," Acta Mater., vol. 76, pp. 94-105, Sep. 2014.

[220] A. J. Bradley and J. W. Rodgers, "The Crystal Structure of the Heusler Alloys," Proc. R. Soc. A Math. Phys. Eng. Sci., vol. 144, no. 852, pp. 340-359, Mar. 1934.

[221] M. F. Ashby and L. M. Brown, "Diffraction contrast from spherically symmetrical coherency strains," Philos. Mag., vol. 8, no. 91, pp. 1083-1103, Jul. 1963.

[222] W. Van den Broek, A. Rosenauer, B. Goris, G. T. Martinez, S. Bals, S. Van Aert, and D. Van Dyck, "Correction of non-linear thickness effects in HAADF STEM electron tomography," Ultramicroscopy, vol. 116, pp. 8-12, May 2012.

[223] A. V. Crewe, J. P. Langmore, and M. S. Isaacson, Physical aspects of electron microscopy and microbeam analysis. New York: Wiley, 1975. 
[224] A. . Ardell, "The effect of volume fraction on particle coarsening: theoretical considerations," Acta Metall., vol. 20, no. 1, pp. 61-71, Jan. 1972.

[225] J. J. Hoyt, "On the steady-state particle size distributions during coarsening," Scr. Metall. Mater., vol. 24, no. 1, pp. 163-166, 1990.

[226] M. Tokuyama and K. Kawazaki, "Statistical-mechanical theory of coarsening of spherical droplets," Phys. A Stat. Mech. its Appl., vol. 123, no. 2-3, pp. 386-411, Feb. 1984.

[227] P. K. Rastogi and A. J. Ardell, "The coarsening behavior of the $\gamma^{\prime}$ precipitate in nickel-silicon alloys," Acta Metall., vol. 19, no. 4, pp. 321-330, Apr. 1971.

[228] D. . Chellman and A. . Ardell, "The coarsening of $\gamma$ ' precipitates at large volume fractions," Acta Metall., vol. 22, no. 5, pp. 577-588, May 1974.

[229] E. Clouet, L. Laé, T. Epicier, W. Lefebvre, M. Nastar, and A. Deschamps, "Complex precipitation pathways in multicomponent alloys.," Nat. Mater., vol. 5, no. 6 , pp. 482-8, Jun. 2006.

[230] V. I. Elagin, V. V. Zakharov, S. G. Pavlenko, and T. D. Rostova, "Influence of Zirconium Additions on Ageing of Al-Sc Alloys," Phys. Met. Met., vol. 60, p. $88,1985$.

[231] C. Fuller, J. Murray, and D. Seidman, "Temporal evolution of the nanostructure of $\mathrm{Al}(\mathrm{Sc}, \mathrm{Zr})$ alloys: Part I - Chemical compositions of $\mathrm{Al}(\mathrm{ScZr})$ precipitates," Acta Mater., vol. 53, no. 20, pp. 5401-5413, Dec. 2005.

[232] A. Tolley, V. Radmilovic, and U. Dahmen, "Segregation in Al3(Sc,Zr) precipitates in Al-Sc-Zr alloys," Scr. Mater., vol. 52, no. 7, pp. 621-625, Apr. 2005.

[233] B. Forbord, W. Lefebvre, F. Danoix, H. Hallem, and K. Marthinsen, "Three dimensional atom probe investigation on the formation of $\mathrm{Al} 3(\mathrm{Sc}, \mathrm{Zr})-$ dispersoids in aluminium alloys," Scr. Mater., vol. 51, no. 4, pp. 333-337, Aug. 2004.

[234] C. B. Fuller, "Temporal Evolution of the Microstructures of Al(Sc,Zr) Alloys and Their Influences on Mechanical Properties," Northwestern Unversity, 2003.

[235] E. Clouet, "Séparation de Phase dans les Alliages Al-Zr-Sc: du Saut des Atomes à la Croissance de Précipités Ordonnés," Feb. 2004.

[236] S. R. Teixeira, P. H. Dionisio, E. F. da Silveria, F. L. Freire, W. H. Schreiner, and I. J. R. Baumvol, "Interdiffusion and reaction in the Fe $\square \mathrm{Al}$ bilayer: I: Rutherford backscattering analysis of furnace-annealed samples," Mater. Sci. Eng., vol. 96, pp. 267-277, Dec. 1987. 
[237] V. P. Shapovalov and A. N. Kurasov, "Diffusion of titanium in iron," Met. Sci. Heat Treat., vol. 17, no. 9, pp. 803-805, Sep. 1975.

[238] K. Nohara and K. Hirano, "Diffusion of manganese into iron," in Proc Int Conf Sci Technol Iron Steel, 1970, p. 11.

[239] F. . Buffington, K. Hirano, and M. Cohen, "Self diffusion in iron," Acta Metall., vol. 9, no. 5, pp. 434-439, May 1961.

[240] A. Takeuchi and A. Inoue, "Classification of Bulk Metallic Glasses by Atomic Size Difference, Heat of Mixing and Period of Constituent Elements and Its Application to Characterization of the Main Alloying Element," Mater. Trans., vol. 46, no. 12, pp. 2817-2829, 2005.

[241] K. Osamura, H. Okuda, K. Asano, M. Furusaka, K. Kishida, F. Kurosawa, and R. Uemori, "SANS Study of Phase Decomposition in Fe-Cu Alloy with Ni and Mn Addition.," ISIJ Int., vol. 34, no. 4, pp. 346-354, May 1994.

[242] C. Zhang and M. Enomoto, "Study of the influence of alloying elements on $\mathrm{Cu}$ precipitation in steel by non-classical nucleation theory," Acta Mater., vol. 54, no. 16, pp. 4183-4191, Sep. 2006.

[243] A. Seko, S. R. Nishitani, I. Tanaka, H. Adachi, and E. F. Fujita, "Firstprinciples calculation on free energy of precipitate nucleation," Calphad, vol. 28, no. 2, pp. 173-176, Jun. 2004.

[244] H. I. Aaronson and F. K. LeGoues, "An assessment of studies on homogeneous diffusional nucleation kinetics in binary metallic alloys," Metall. Trans. A, vol. 23, no. 7, pp. 1915-1945, Jul. 1992.

[245] Q. Furnemont, M. Kempf, P. J. Jacques, M. Goken, and F. Delannay, “On the measurement of the nanohardness of the constitutive phases of TRIP-assisted multiphase steels," Mater. Sci. Eng. A, vol. 328, no. 1, pp. 26-32.

[246] P. W. Hochanadel, C. V. Robino, G. R. Edwards, and M. J. Cieslak, "Heat treatment of investment cast PH 13-8 Mo stainless steel: Part I. Mechanical properties and microstructure," Metall. Trans. A, Phys. Metall. Mater. Sci., vol. 25 A, no. 4, pp. 789-798, 1994.

[247] J. M. Cloué, B. Viguier, and E. Andrieu, "Effect of the metallurgical variables on the yield stress of PH 13-08 steel," Metall. Mater. Trans. A Phys. Metall. Mater. Sci., vol. 36, no. 10, pp. 2633-2639, 2005.

[248]R. Ebeling and M. F. Ashby, "Dispersion hardening of copper single crystals," Philos. Mag., vol. 13, no. 124, pp. 805-834, Apr. 1966. 
[249] W. D. Nix and H. Gao, "Indentation size effects in crystalline materials: A law for strain gradient plasticity," J. Mech. Phys. Solids, vol. 46, no. 3, pp. 411-425, Mar. 1998.

[250] K. Durst, B. Backes, O. Franke, and M. Göken, "Indentation size effect in metallic materials: Modeling strength from pop-in to macroscopic hardness using geometrically necessary dislocations," Acta Mater., vol. 54, no. 9, pp. 2547-2555, May 2006.

[251] P. C. Wo and A. H. W. Ngan, "Investigation of slip transmission behavior across grain boundaries in polycrystalline Ni3Al using nanoindentation," $J$. Mater. Res., vol. 19, no. 1, pp. 189-201, 2004.

[252] H. Mehrer, Ed., Diffusion in Solid Metals and Alloys, vol. 26. Berlin/Heidelberg: Springer-Verlag, 1990.

[253] K. Ishida, "Calculation of the effect of alloying elements on the Ms temperature in steels," J. Alloys Compd., vol. 220, no. 1-2, pp. 126-131, 1995.

[254] J. Han, S.-J. Lee, J.-G. Jung, and Y.-K. Lee, "The effects of the initial martensite microstructure on the microstructure and tensile properties of intercritically annealed Fe-9Mn-0.05C steel," Acta Mater., vol. 78, pp. 369377, Oct. 2014.

[255] T. Furukawa, H. Huang, and O. Matsumura, "Effects of carbon content on mechanical properties of $5 \% \mathrm{Mn}$ steels exhibiting transformation induced plasticity," Mater. Sci. Technol., vol. 10, no. 11, p. 964, Jul. 1994.

[256] G. . Ansell and F. . Lenel, "Criteria for yielding of dispersion-strengthened alloys,” Acta Metall., vol. 8, no. 9, pp. 612-616, Sep. 1960.

[257] G. S. Ansell, "Fine particle effect in dispersion-strengthening," Acta Metall., vol. 9, no. 5, pp. 518-519, May 1961. 


\section{Appendix Crystallographic information of the phases in maraging steels}

\begin{tabular}{|c|c|c|c|c|c|c|c|c|}
\hline \multirow{2}{*}{ Phase } & \multirow{2}{*}{ Formula } & \multirow{2}{*}{$\begin{array}{l}\text { Crystal } \\
\text { structure }\end{array}$} & \multirow{2}{*}{$\begin{array}{c}\text { Lattice } \\
\text { parameter }(\mathrm{nm})\end{array}$} & \multirow{2}{*}{ Space group } & \multicolumn{4}{|c|}{ Atomic position } \\
\hline & & & & & Atom & $X$ & $\mathrm{Y}$ & $\mathrm{Z}$ \\
\hline \multirow[t]{2}{*}{$\alpha$} & \multirow[t]{2}{*}{$\mathrm{Fe}_{83.5} \mathrm{Mn}_{16.5}$} & \multirow[t]{2}{*}{ bcc } & \multirow[t]{2}{*}{$\mathrm{a}=0.2884$} & \multirow[t]{2}{*}{$\operatorname{Im} \overline{3} m$ (229) } & $\mathrm{Fe}$ & 0 & 0 & 0 \\
\hline & & & & & Mn & 0 & 0 & 0 \\
\hline \multirow[t]{2}{*}{$\gamma$} & \multirow[t]{2}{*}{$\mathrm{Fe}_{65} \mathrm{Mn}_{35}$} & \multirow[t]{2}{*}{ fcc } & \multirow[t]{2}{*}{$\mathrm{a}=0.3605$} & \multirow[t]{2}{*}{$F m \overline{3} m(225)$} & $\mathrm{Fe}$ & 0 & 0 & 0 \\
\hline & & & & & Mn & 0 & 0 & 0 \\
\hline \multirow[t]{2}{*}{$\varepsilon$} & \multirow[t]{2}{*}{$\mathrm{Fe}_{83.5} \mathrm{Mn}_{16.5}$} & \multirow[t]{2}{*}{ hexagonal } & \multirow{2}{*}{$\begin{array}{l}a=0.2536 \\
c=0.4089\end{array}$} & \multirow[t]{2}{*}{$\mathrm{P} 63 / \mathrm{mmc}(194)$} & $\mathrm{Fe}$ & $1 / 3$ & $2 / 3$ & $1 / 4$ \\
\hline & & & & & $\mathrm{Mn}$ & $1 / 3$ & $2 / 3$ & $1 / 4$ \\
\hline \multirow[t]{2}{*}{$\eta$-NiAl } & \multirow[t]{2}{*}{$\mathrm{NiAl}$} & \multirow[t]{2}{*}{ B2 } & \multirow[t]{2}{*}{$\mathrm{a}=0.2887$} & \multirow[t]{2}{*}{$P m \overline{3} m$ (221) } & $\mathrm{Ni}$ & 0 & 0 & 0 \\
\hline & & & & & $\mathrm{Al}$ & $1 / 2$ & $1 / 2$ & $1 / 2$ \\
\hline
\end{tabular}


Appendix 1 (continued)

\begin{tabular}{|c|c|c|c|c|c|c|c|c|}
\hline \multirow{2}{*}{ Phase } & \multirow{2}{*}{ Formula } & \multirow{2}{*}{$\begin{array}{l}\text { Crystal } \\
\text { structure }\end{array}$} & \multirow{2}{*}{$\begin{array}{c}\text { Lattice } \\
\text { parameter }(\mathrm{nm})\end{array}$} & \multirow{2}{*}{ Space group } & \multicolumn{4}{|c|}{ Atomic position } \\
\hline & & & & & Atom & $X$ & $\mathrm{Y}$ & $\mathrm{Z}$ \\
\hline \multirow{3}{*}{$\mathrm{Ni}_{2} \mathrm{TiAl}$} & \multirow{3}{*}{$\mathrm{Ni}_{2} \mathrm{TiAl}$} & \multirow{3}{*}{$\mathrm{L} 2_{1}$} & \multirow{3}{*}{$a=0.585$} & \multirow{3}{*}{$F m \overline{3} m(225)$} & $\mathrm{Al}$ & 0 & 0 & 0 \\
\hline & & & & & $\mathrm{Ti}$ & $1 / 2$ & $1 / 2$ & $1 / 2$ \\
\hline & & & & & $\mathrm{Ni}$ & $1 / 4$ & $1 / 4$ & $1 / 4$ \\
\hline
\end{tabular}

